UNIVERSITY OF SALAMANCA

FACULTY OF PHARMACY

DEPARTMENT OF PHYSIOLOGY AND PHARMACOLOGY

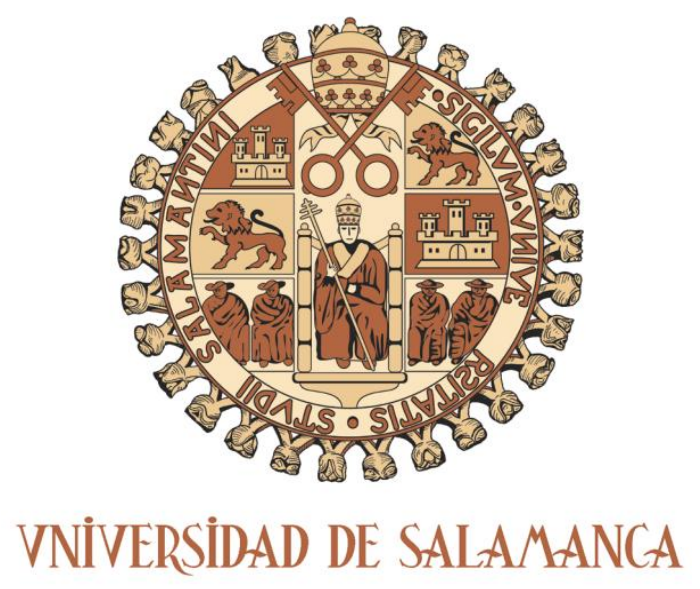

CHARACTERIZATION OF PHENOLIC CONSTITUENTS OF MEDICINAL PLANTS AND EVALUATION OF PHARMACOLOGICAL ACTIVITIES: FOCUS IN ANTIOXIDANT AND ANTI-INFLAMMATORY PROPERTIES

DOCTORAL THESIS

OLÍVIA RODRIGUES PEREIRA 


\section{UNIVERSITY OF SALAMANCA}

FACULTY OF PHARMACY

DEPARTMENT OF PHYSIOLOGY AND PHARMACOLOGY

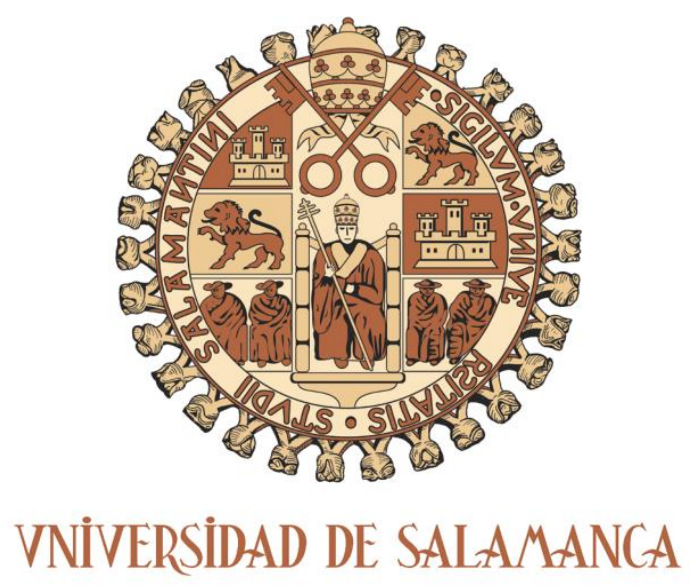

\section{CHARACTERIZATION OF PHENOLIC CONSTITUENTS OF MEDICINAL PLANTS AND EVALUATION OF PHARMACOLOGICAL ACTIVITIES: FOCUS IN ANTIOXIDANT AND ANTI-INFLAMMATORY PROPERTIES}

Doctoral Dissertation presented by Olívia Rodrigues Pereira for PhD degree of University of Salamanca

Supervisor of the thesis:

Susana Maria de Almeida Cardoso

Tutor of the thesis:

José Juan García Marín

Salamanca, $19^{\text {th }}$ June 2013 
Dạ. Ma JESÚS MONTE RÍO, DIRECTORA DEL DEPARTAMENTO DE FISIOLOGÍA Y FARMACOLOGÍA DE LA UNIVERSIDAD DE SALAMANCA

\section{CERTIFICA:}

Que la Memoria titulada "CHARACTERIZATION OF PHENOLIC CONSTITUENTS OF MEDICINAL PLANTS AND EVALUATION OF PHARMACOLOGICAL ACTIVITIES: FOCUS IN ANTIOXIDANT AND ANTI-INFLAMMATORY PROPERTIES", presentada por $D^{\mathrm{a}}$. Olívia Rodrigues Pereira para optar al Título de Doctor por la Universidad de Salamanca, ha sido realizada bajo la dirección de la Drạ. Dạ . Susana Maria de Almeida Cardoso, Investigadora Auxiliar en el Centro de Estudos de Recursos Naturais, Ambiente e Sociedade y la tutela del Dr. D. José Juan García Marín, Catedrático del Departamento de Fisiología y Farmacología de la Universidad de Salamanca.

Y para que así conste, expide y firma la presente certificación en Salamanca a día diecinueve de junio de dos mil trece.

Fdo. Ma Jesús Monte Río 
DÑA SUSANA MARIA DE ALMEIDA CARDOSO, INVESTIGADORA AUXILIAR DEL CENTRO DE ESTUDOS DE RECURSOS NATURAIS, AMBIENTE E SOCIEDADE Y D. JOSÉ JUAN GARCÍA MARÍN CATEDRÁTRICO DEL DEPARTAMENTO DE FISIOLOGÍA Y FARMACOLOGÍA DE LA UNIVERSIDAD DE SALAMANCA

CERTIFICAN:

Que la Memoria titulada "CHARACTERIZATION OF PHENOLIC CONSTITUENTS OF MEDICINAL PLANTS AND EVALUATION OF PHARMACOLOGICAL ACTIVITIES: FOCUS IN ANTIOXIDANT AND ANTI-INFLAMMATORY PROPERTIES", presentada por $D^{a}$. Olívia Rodrigues Pereira para optar al Título de Doctor por la Universidad de Salamanca, ha sido realizada bajo la dirección de la Dra . Da. Susana Maria de Almeida Cardoso y la tutela del Dr. D. José Juan García Marín. El trabajo experimental ha sido desarrollado en el Laboratório de Química e Bioquímica Aplicada del Centro de Investigação em Montanha (CIMO) en Bragança, en el Centro de Estudos de Recursos Naturais, Ambiente e Sociedade (CERNAS) en Coimbra, en el Departamento de Química de Universidade de Aveiro y en el Laboratorio de Hepatología Experimental y Vectorización de Fármacos (HEVEFARM) del Departamento de Fisiología y Farmacología de la Universidad de Salamanca.

Y para que así conste, expiden y firman la presente certificación en Salamanca a día diecinueve de junio de dos mil trece.

Fdo. Susana Maria de Almeida Cardoso

Fdo. José Juan García Marín 


\section{ACKNOWLEDGEMENTS}

I would like to thank my supervisor, Doctor Susana M. Cardoso, for her encouragement, sound advice, good teaching, good ideas and for the active and incessant guidance. I am also thankful for having me shown that the world of research is so tiring as exciting.

I also would like to extend my appreciation and gratitude to my tutor, Doctor José Juan García Marín, for giving me the possibility to join his investigation group and for his valuable advices. I am thankful to Doctor $\mathrm{M}^{\mathrm{a}}$ Ángeles Serrano for her support in the initial stage of my work and also to Doctor $\mathrm{M}^{\mathrm{a}}$ José Pérez and Doctor Rocío Macías for their guidance in the laboratory.

I also thank the collaboration of Doctor $\mathrm{M}^{\mathrm{a}}$ Rosário Domingues for welcoming me in her laboratory, for introducing me in mass spectrometry technique, monitoring in data interpretation and for her constant support.

Furthermore, I want to express my thanks to Doctor Artur M. Silva, Doctor Fernanda Ferreira and Mrs Susana Saraiva for their valuable contribution in NMR, mitochondrial bioenergetics and anti-inflammatory experiments, respectively.

I address my warm thanks to all my former and current colleagues of the several laboratories where the work was developed and also to my friends of the school where I daily work for all support, fellowship, entertainment, and caring they provided.

I dedicate my work to all my family in particular to my sister Marta and to my parents $\mathrm{M}^{\mathbf{a}}$ Eduarda e Octávio and to Marco for their unconditional support.

I thank all those who helped the implementation of the work.

Thank You!

The work presented in this Doctoral Thesis was supported by a PhD grant SFRH/PROTEC/49600/2009. 


\section{TABLE OF CONTENTS}

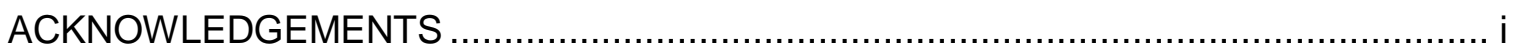

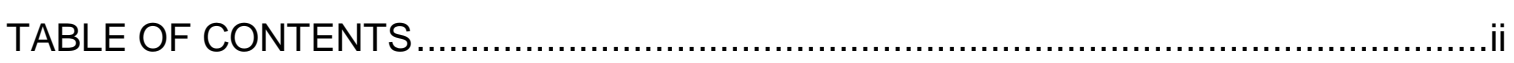

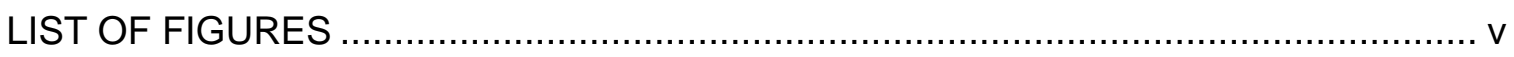

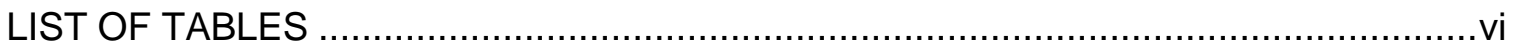

LIST OF ABBREVIATIONS............................................................................

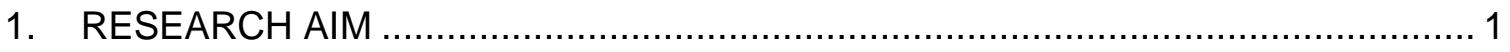

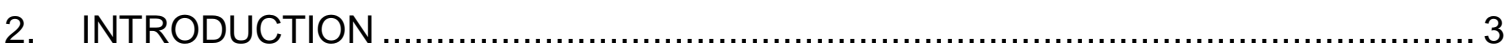

2.1. General description of plants .............................................................. 5

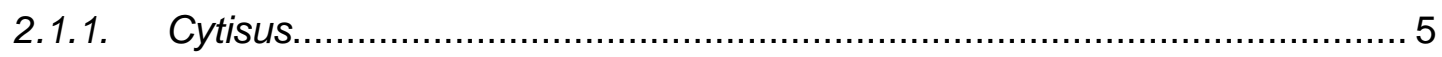

2.1.1.1. Cytisus multiflorus ............................................................... 5

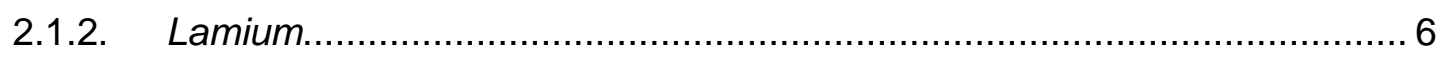

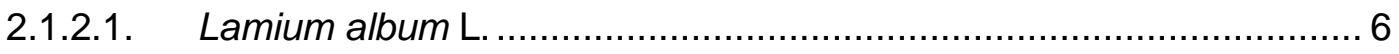

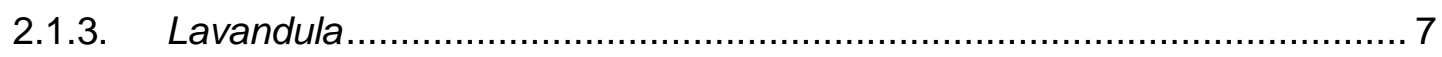

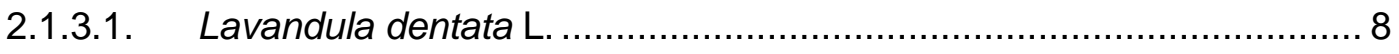

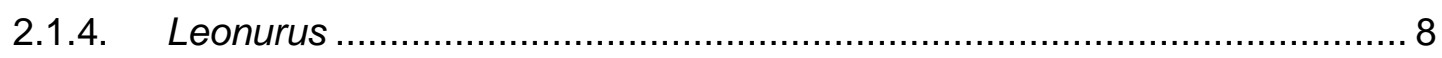

2.1.4.1. Leonurus cardiaca L. ................................................................ 9

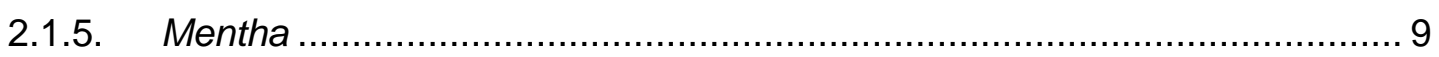

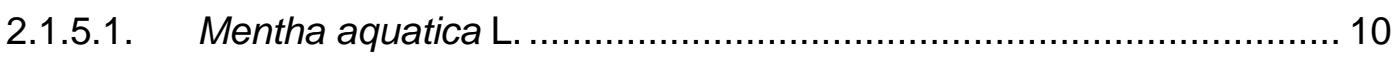

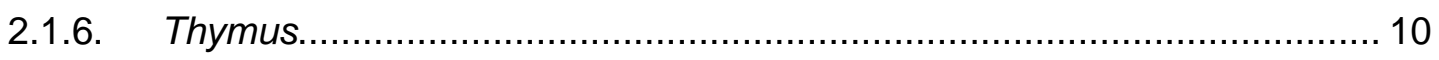

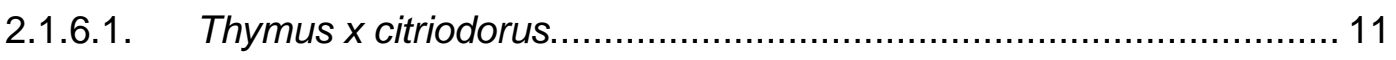

2.2. Methods of extraction, purification and characterization of plant phenolics ..... 12

2.2.1. Sample preparation and extraction .................................................... 12

2.2.2. Clean-up and fractionation............................................................. 13

2.2.3. Detection and characterization of phenolic compounds ......................... 13

2.3. Phenolic compounds in the target plant genus............................................ 16

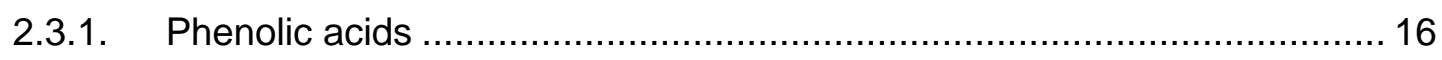

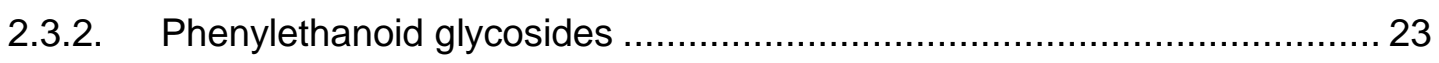

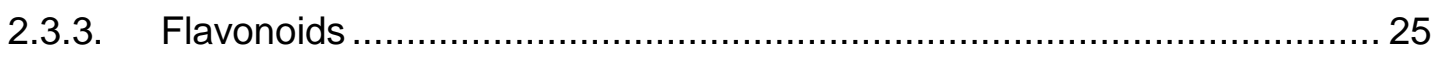

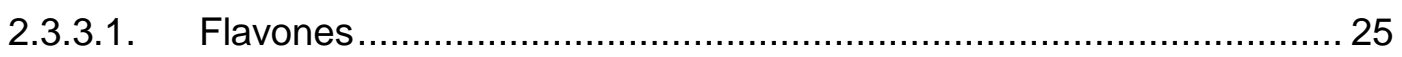

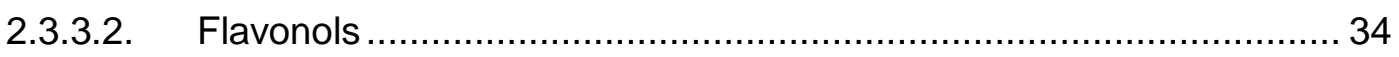

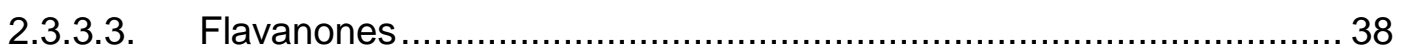

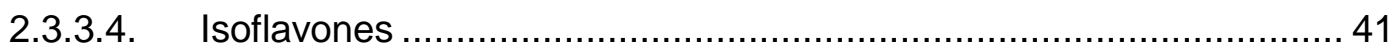

2.4. Beneficial effects ............................................................................ 42 


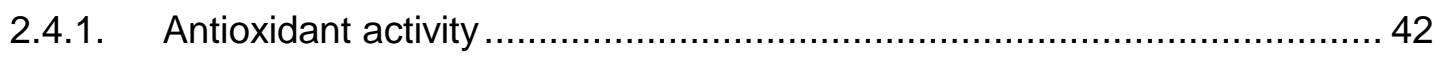

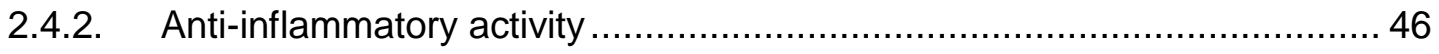

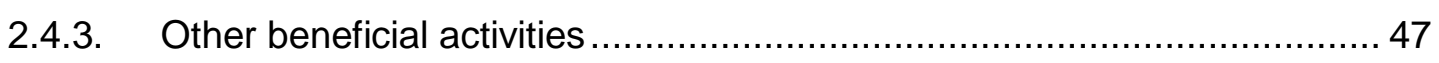

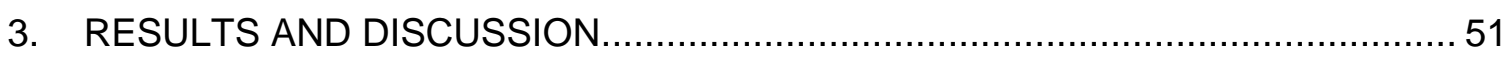

3.1. Simultaneous characterization and quantification of phenolic compounds in Thymus $x$ citriodorus using a validated HPLC-UV and ESI-MS combined method ....54

3.2. Identification of phenolic constituents of Cytisus multiflorus ...........................55

3.3. Phenolic constituents of Lamium album: focus on isoscutellarein derivatives . 56

3.4. Phenolic characterization of Leonurus cardiaca L. extracts........................... 57

3.4.1. Materials and Methods .............................................................. 57

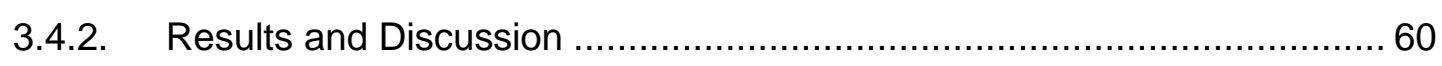

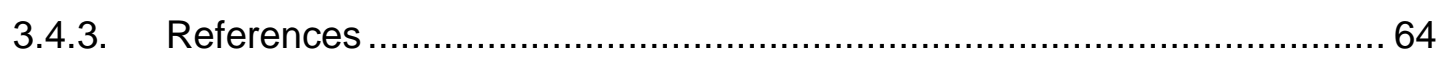

3.5. ROS scavenging and hepatoprotective activities of Mentha aquatica L. and

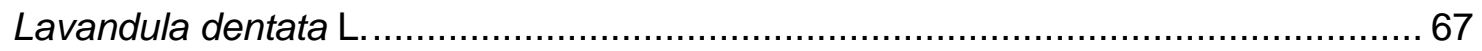

3.6. Protective effects of phenolic constituents from Cytisus multiflorus, Lamium

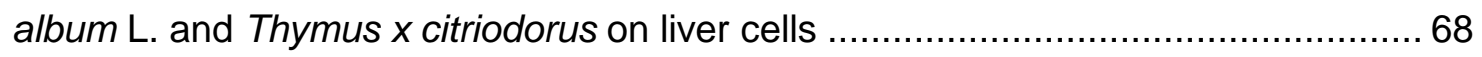

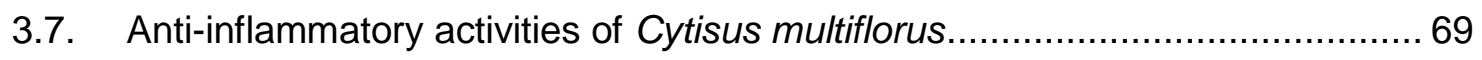

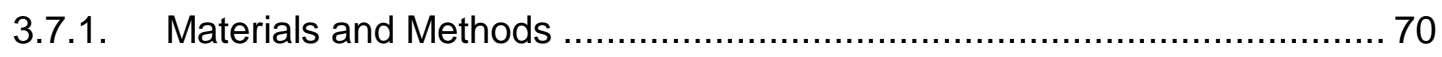

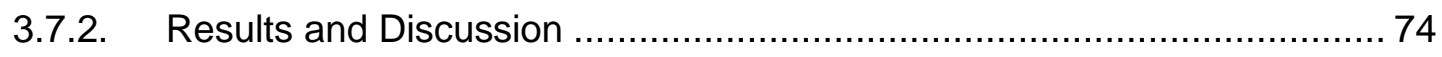

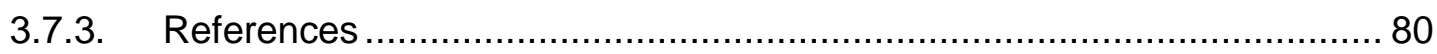

3.8. Influence of Mentha aquatica L. and Leonurus cardiaca L. purified ethanolic extracts in mitochondrial bioenergetics ............................................................... 83

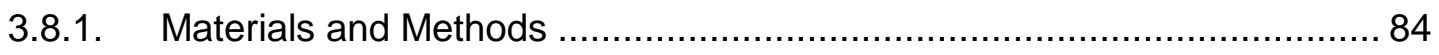

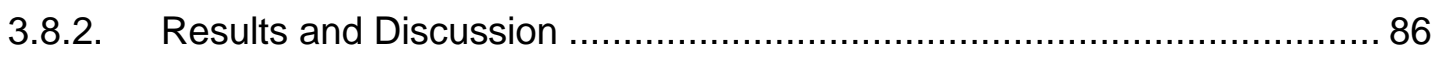

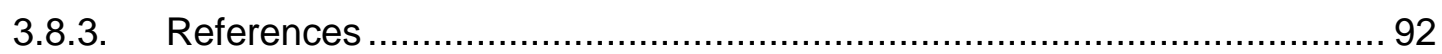

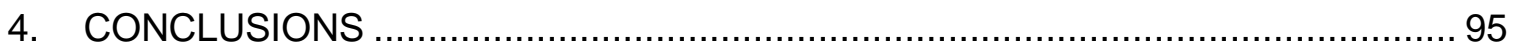

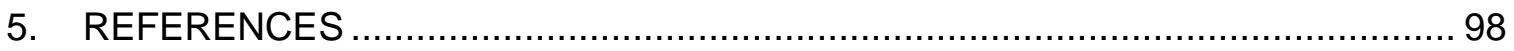

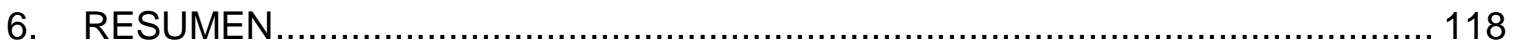

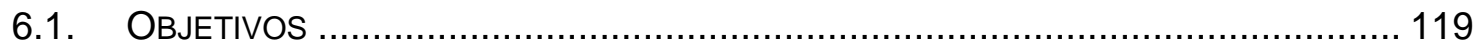

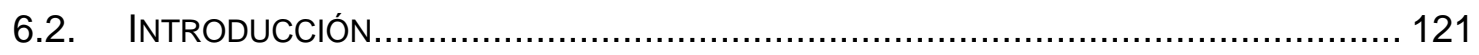

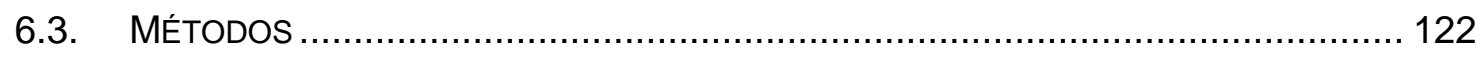

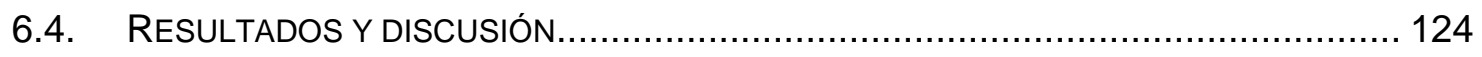

6.4.1. Caracterización y cuantificación de compuestos fenólicos presentes en Thymus $x$ citriodorus usando un método validado de HPLC-UV y ESI-MS .......... 124

6.4.2. Identificación de compuestos fenólicos de Cytisus multiflorus ................ 129

6.4.3. Compuestos fenólicos de Lamium album L.: derivados de isoscutelareína 132 
6.4.4. Caracterización fenólica de extractos de Leonurus cardiaca L.

6.4.5. Actividades hepatoprotectora y de captura de ROS por Mentha aquatica L. y Lavandula dentata $L$ 137

6.4.6. Efectos protectores en células hepáticas de los compuestos fenólicos presentes en Cytisus multiflorus, Lamium album L. y Thymus $x$ citriodorus......... 140

6.4.7. Efecto anti-inflamatorio de Cytisus multiflorus....................................... 144

6.4.8. Influencia de los extractos etanólicos purificados de Mentha aquatica L. y Leonurus cardiaca L. en la bioenergética mitocondrial..................................... 147

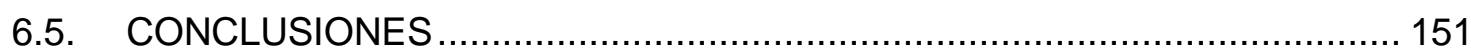




\section{LIST OF FIGURES}

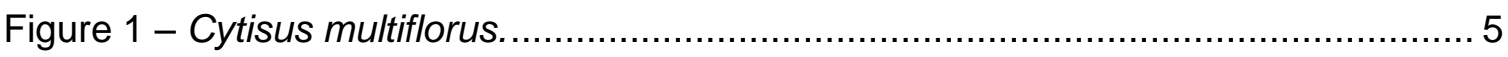

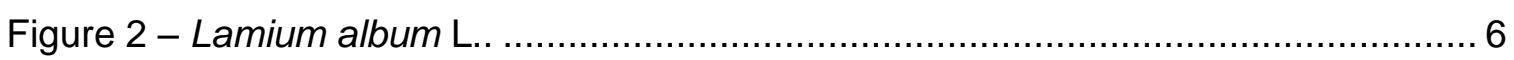

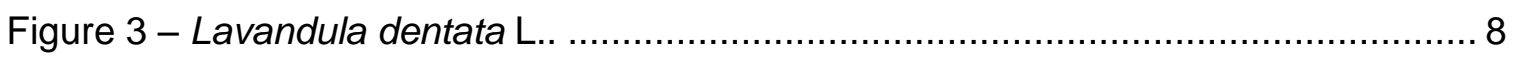

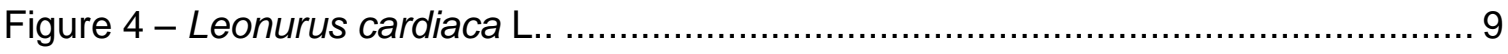

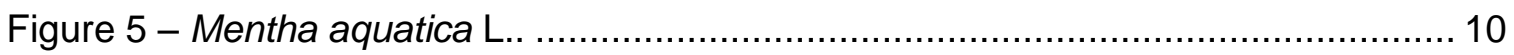

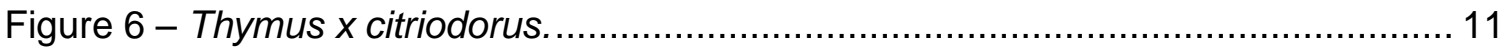

Figure 7 - General structure of hydroxybenzoic (A) and hydroxycinnamic (B) acids.... 16

Figure 8 - Chemical structures of the phenolic acids reported in Cytisus, Lamium, Lavandula, Leonurus, Mentha and Thymus plants................................................. 22

Figure 9 - General structure of phenylethanoid glycosides................................... 23

Figure 10 - Chemical structures of phenylethanoid glycosides reported in Lamium and

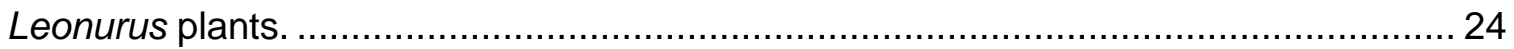

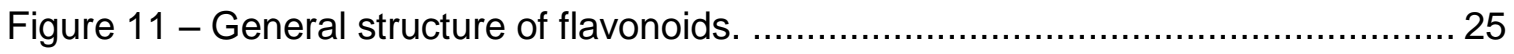

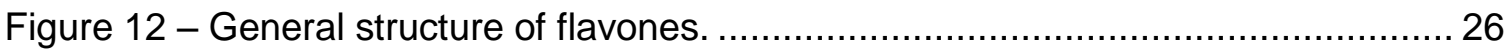

Figure 13 - Chemical structures of flavones reported in Cytisus, Lamium, Lavandula, Leonurus, Mentha and Thymus plants. ............................................................... 33

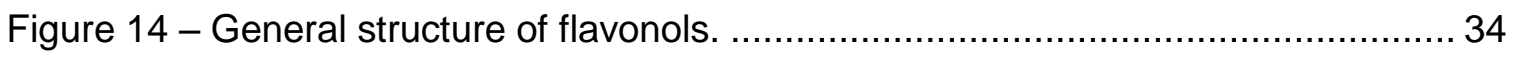

Figure 15 - Chemical structures of flavonols reported in Cytisus, Lamium, Lavandula,

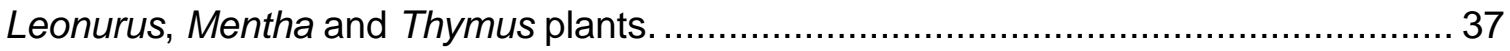

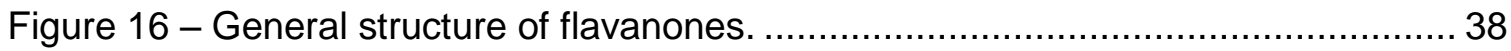

Figure 17 - Chemical structures of flavanones reported in Lavandula, Leonurus, Mentha

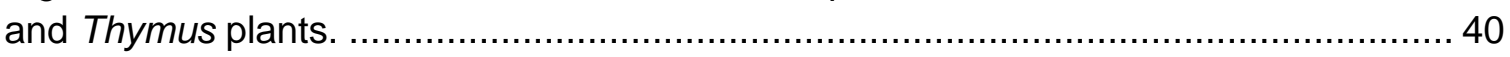

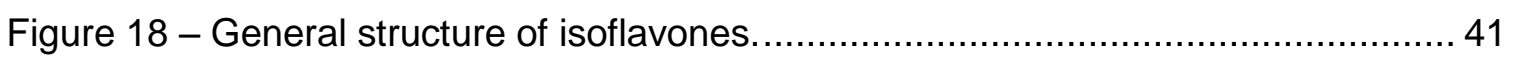

Figure 19 - Chemical structures of isoflavones reported in Cytisus plants................... 41 


\section{LIST OF TABLES}

Table 1 - Phenolic acids of Cytisus, Lamium, Lavandula, Leonurus, Mentha and Thymus

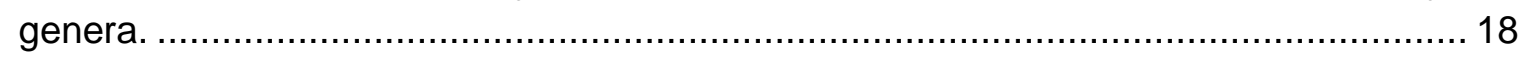

Table 2 - Phenylethanoid glycosides of Lamium and Leonurus genera........................ 24

Table 3 - Flavones of Cytisus, Lamium, Lavandula, Leonurus, Mentha and Thymus

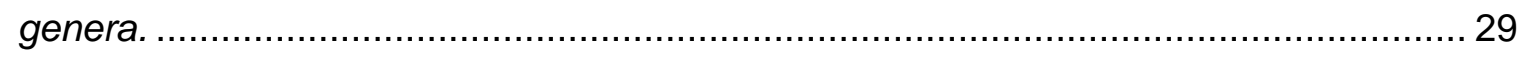

Table 4 - Flavonols of Cytisus, Lamium, Lavandula, Leonurus, Mentha and Thymus

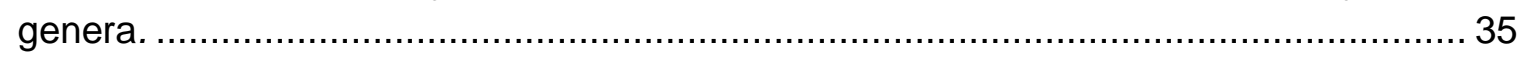

Table 5 - Flavanones of Lavandula, Leonurus, Mentha and Thymus genera. ............... 40

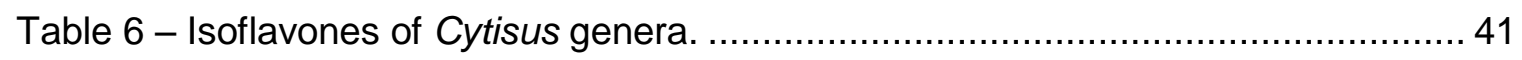

Table 7 - Described effects in Cytisus, Lamium, Lavandula, Leonurus, Mentha and

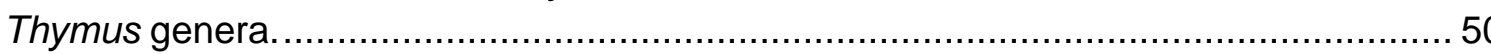




\section{LIST OF ABBREVIATIONS}

AAE Ascorbic acid equivalent

ABTS 2,2'-azino-bis-3-ethylbenzthiazoline-6-sulphonic acid

AChE Acetylcholinesterase

ADP Adenosine diphosphate

ATP Adenosine triphosphate

BChE Butyrylcholinesterase

BHA Butylated hydroxyanisole

CAT Catalase

CME Cytisus multiflorus extract

COX-1 Cyclooxygenase-1

COX-2 Cyclooxygenase-2

Da Dalton

DAD Diode array detection

DCF Dichlorofluorescein

$\mathrm{DCFH}_{2}$ Dihydrodichlorofluorescein diacetate

DK Potassium dichromate

DNA Deoxyribonucleic acid

DPPH $^{\bullet}$ Radical 2,2-diphenyl-1-picrylhydrazyl

$\mathrm{EC}_{50}$ Half maximal effective concentration

ESI Electrospray ionization

FCCP Carbonyl cyanide $p$-trifluoromethoxyphenylhydrazone

FRAP Reducing/antioxidant power

GABA $_{A} \quad \mathrm{Y}$-aminobutyric acid A

GAE Gallic acid equivalents

GPx Glutathione peroxidase

GRD Glutathione reductase

GSH Reduced glutathione

GSSG Oxidized glutathione

GST Glutathione-s-transferase

HDL High-density lipoprotein

$\mathrm{HOCl}$ Hypochlorous acid

HPLC High performance liquid chromatography

IL Interleukins

INF Interferon

iNOS Inducible nitric oxide synthase

LC Liquid chromatography

LD Detection limit

LDH Lactate dehydrogenase 
LOX Lipoxygenase

LPS Lipopolysaccharide

LQ Quantification limit

MAO-A Monoamino oxidase A

MS Mass spectrometry

MS $^{n}$ Tandem mass spectrometry

MTT 3-(4,5-dimethylthiazol-2-yl)-2,5-diphenyl tetrazolium bromide

Nf-kB Nuclear factor-kB

NMR Nuclear magnetic resonance

NO• Nitric oxide radical

Nrf-2 NF-E2-related factor 2

$\mathrm{O}_{2}{ }^{\bullet-}$ Superoxide radical

$\mathrm{OH}^{\bullet}$ Hydroxyl radical

$\mathrm{ONOO}^{-}$Peroxynitrite anion

ORAC Oxygen radical absorbance capacity $\mathrm{P} / \mathrm{O}$ ratio $\begin{aligned} & \text { Ratio of ADP molecules phosphorylated to atoms of } \\ & \text { oxygen consumed }\end{aligned}$

PBQ $p$-benzoquinone

PDA Photodiode array detector

PEELC L. cardiaca purified ethanolic extract

PEEMa $M$. aquatica purified ethanolic extract

$\mathrm{PGE}_{2}$ Prostaglandin $\mathrm{E}_{2}$

$\mathrm{RCR}$ Respiratory control ratio

RNS Reactive nitrogen species

ROS Reactive oxygen species

RT Retention time

SD Standard deviation

SEM Standard error of the mean

SGOT Glutamate oxaloacetate transaminases

SOD Superoxide dismutase

TBARS Thiobarbituric acid reactive substances

TEAC Trolox equivalence antioxidant capacity

TNF- $\alpha$ Tumor necrosis factor alfa

TGF- $\beta$ Transforming growth factor beta

UV Ultraviolet

$\Delta \psi$ Mitochondrial transmembrane potential 
1. RESEARCH AIM 
The polyphenolic profiles of the plant species Cytisus multiflorus, Lamium album L., Lavandula dentata L., Leonurus cardiaca L., Mentha aquatica L. and Thymus $x$ citriodorus are poorly studied or even still unknown, and thus more studies are necessary. In a same way, several properties have been assigned to different plant extracts, however scientific investigations are needed to prove the beneficial properties in human health since, in most cases, the biological effects have been exclusively tested in in vitro models.

In this context, the present Doctoral Thesis intended to improve the knowledge of the phenolic composition and also of the beneficial effects of six medicinal plants namely Cytisus multiflorus, Lamium album L., Lavandula dentata L., Leonurus cardiaca L., Mentha aquatica $L$. and Thymus $x$ citriodorus. For that, five specific aims were defined:

First aim: Characterize and quantify the phenolic constituents of Cytisus multiflorus, Lamium album L., Lavandula dentata L., Leonurus cardiaca L., Mentha aquatica L. and Thymus $x$ citriodorus by high performance liquid chromatography associated with diode array detection (HPLC-DAD), electrospray mass spectrometry (ESI-MS and MS ${ }^{n}$ ) and nuclear magnetic resonance (NMR) techniques;

Second aim: Determine antioxidant effects of Cytisus multiflorus, Lamium album L., Lavandula dentata L., Leonurus cardiaca L., Mentha aquatica L. and Thymus $x$ citriodorus in chemical and cellular models;

Third aim: Evaluate the hepatoprotective effects of Cytisus multiflorus, Lamium album L., Lavandula dentata L., Leonurus cardiaca L., Mentha aquatica L. and Thymus $x$ citriodorus in human hepatoblastoma HepG2 cells;

Fourth aim: Evaluate the anti-inflammatory properties of Cytisus multiflorus extract;

Fifth aim: Evaluate the influence of Mentha aquatica L. and Leonurus cardiaca L. plant extracts in mitochondria bioenergetics. 
2. INTRODUCTION 
In recent years, several beneficial activities of plants have been attributed to their polyphenolic composition [1]. On the other hand, Mediterranean region is rich in medicinal plants both in wild or cultivated forms that mainly because of their medicinal value, are potential candidates for exploitation by various industries, including the food and cosmetic industries.

This first section of the Doctoral Thesis aimed to introduce the subject of the present work. For that, a general description of the six plant genus together with the target plants is made, followed by an overview on the methods of extraction and characterization of phenolic compounds, in particular those performed with the six target plant genus. Moreover, the phenolic compounds of these genera, as well as the potential biological effects reported in the literature up to this moment, are summarized.

Part of the information presented in this section has been used to write the manuscript "Overview on Mentha and Thymus polyphenols" which is published in Current Analytical Chemistry: Pereira, O. R.; Cardoso, S. M., Overview on Mentha and Thymus Polyphenols. Current Analytical Chemistry, 2013, 9(3), 382-396. 


\subsection{GenERAL DESCRIPTION OF PLANTS}

\subsubsection{Cytisus}

Cytisus Desf. (Leguminosae - Cytiseae) is a genus of flowering plants belonging to the Fabaceae family [2]. The species of this genus occur as unarmed shrubs with twigs ribbed and alternate, the leaves are persistent, trifoliolate and petiolate. The inflorescences are characterized by $1-3$ flowers in axillary fascicles $[2,3]$.

Several species of the Cytisus genus are used in traditional medicine mainly due to their anxiolytic, antidiabetic, antioxidant, diuretic, hypnotic and antiparasitic properties. [4-10].

\subsubsection{Cytisus multiflorus}

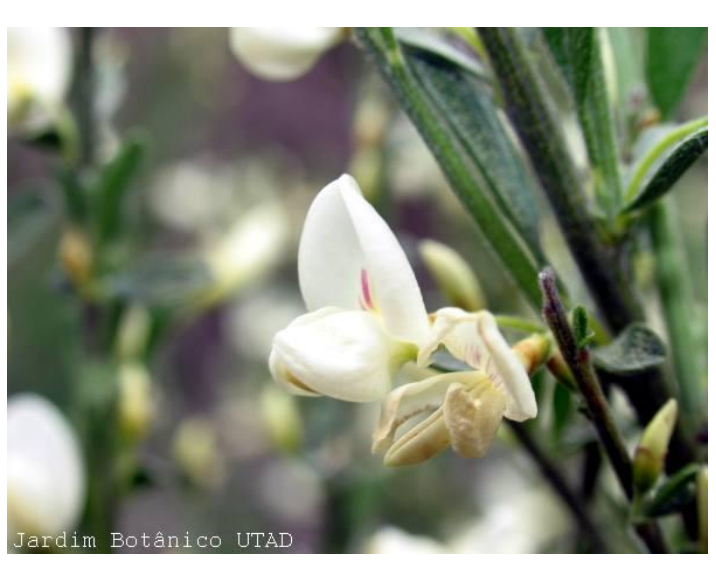

Figure 1 - Cytisus multiflorus.

Jardim Botânico da UTAD (2013), Retrieved from:

<http://jb.utad.pt/especie/cytisus_multiflorus>
Cytisus multiflorus (L'Hér.) Sweet (Fig. 1), or white Spanish broom, is one of the approximately 60 species from the Cytisus genus. This species is endemic from southwest Mediterranean region and it is largely distributed in the Iberian Peninsula [3]. The plant frequently appears as a shrub, covering extensive areas of degraded and marginal soils. It is a large and upright broom with small leaves and a great number of white flowers with a valvular type pollen presentation system [11].

C. multiflorus species is vastly used as an ornamental plant, as well as for animal nutrition. Other applications of the plant include the collection of their pollen for apiculture purposes and land fertilizing in agriculture [11-14]. Besides these, the plant has also been used for centuries in the form of tea for the treatment of metabolic and endocrine system disorders and as an antiinflammatory, diuretic and anti-hypertensor agent $[15,16]$. 


\subsubsection{Lamium}

Plants of Lamium L. (Family: Lamiaceae alt. Labiatae) genus are native of the Old World and distributed in Europe, Asia, and Africa. They are globally known as "dead nettle" due to the absence of stinging hairs, which are typically found in stinging nettles [17]. The Lamium genus comprises about 40 annual or perennial herb species with verticillasters, a whorled inflorescence dense or remote and 2 to 12 flowered. The calyx is cylindrical or campanulate, 5-nerved, 5-toothed and the corolla bilabiate can be purple, pink, cream or white. The leaves are ovate to reniform and crenate to dentate and the fruits can appear as trigonous nutlets, truncate at apex [17].

Many species of this genus have been used as famine food in starvation periods, such as war periods. Moreover, some Lamium species are considered as medicinal plants due to antioxidant, antispasmodic, anti-inflammatory and antinconceptive properties, as well as to their ability to treat musculoskeletal and several gynecological conditions [18, 19].

\subsubsection{Lamium album $L$.}

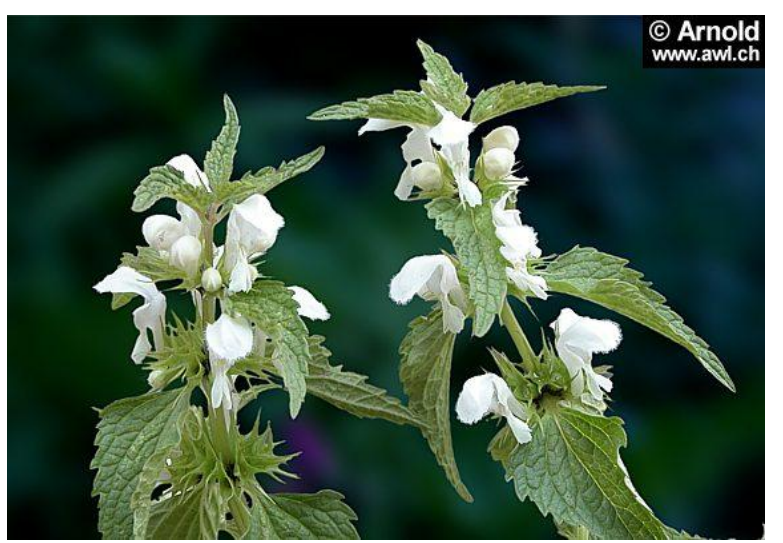

Figure 2 - Lamium album L..

Arnold, W. (2013), Retrieved from: http://www.awl.ch/heilpflanzen/lamium_album/
Lamium album L. (Fig. 2), commonly known as white dead nettle, is consumed in the Mediterranean and surrounding areas for confection of local dishes [20]. In fact, the young shoots, leaves and flowers of this plant are edible and consumed raw or cooked as a vegetable. The plant is also commonly used as an ingredient in several dishes including omelets, stews and roasts [21]. Moreover, white dead nettle is the base ingredient for important vegetarian dishes such as the "White Dead Nettle Frittata", "White Dead Nettle, Feta \& Watermelon Salad" and the "Deadnettle soup" [22, 23]. The flowers of this plant are very attractive to bees and other pollinating insects and hence, these have been frequently used for honey production [24, 25]. The species is also used in teas and in food supplements preparations, which consumption is primarily associated to the plant health benefits. In fact, L. album is famous due to its antioxidant, antispasmodic and mucolytic (useful in chronic bronchitis or pharyngitis), diuretic, astringent and anti-septic 
activities. Additionally, the aerial parts of this plant are often used in the treatment of menorrhagia, uterine hemorrhage, vaginal and cervical inflammation, leucorrhoea, wound healing and skin problems because of its haemostatic and anti-inflammatory activities [26, 27]. Besides this, the consumption of food supplements enriched in $L$. album extracts are claimed to detoxify the organism, to prevent menstrual disorders, abdominal inflammation and musculoskeletal diseases [28], and to improve the fat metabolism [29].

\subsubsection{Lavandula}

Lavandula L. genus (Lamiaceae) comprises about 30 aromatic annual species which are used since ancient times for medicinal, ornamental and melliferous purposes. The different species appear as small shrubs or herbaceous plants and are endemic in the Mediterranean region, tropical Africa and southeast of India [30].

Lavender plants are particularly known for their essential oils, which have a high commercial value. As a consequence of that, several lavender species are largely cultivated in France, Italy and Spain [30] for oil extraction, to be used in perfumery industry or for producing other cosmetics, like skin lotions, colognes and soaps. More recent applications of lavender oils include aromatherapy and massages and food flavoring. Examples of the latter are its usage in the manufacture of ice cream, candy, chewing gum and beverages [31-36]. Besides those, several Lavandula species are used as medicinal plants. This includes their usage to combact painful conditions and digestive complaints. Moreover, they are claimed to act as antidiabetic, antidepressant, sedative, local anesthetic, antispasmodic, antimicrobial, and antiparasitic agents. There are also some evidences of possibly effectiveness in hair loss in alopecia areata condition [35, 37-39]. 


\subsubsection{Lavandula dentata $L$.}

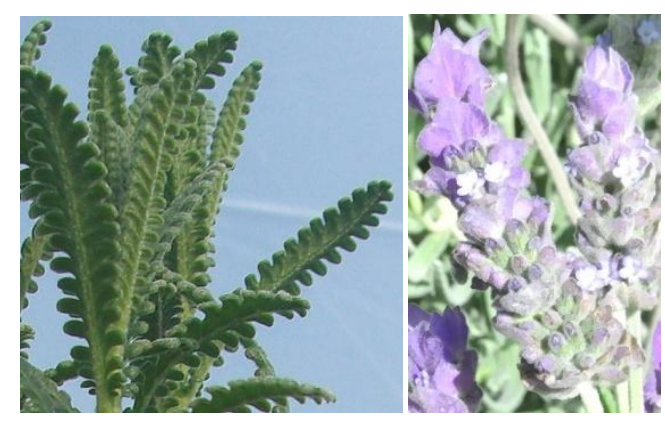

Figure 3 - Lavandula dentata L..

Mountain Valley Growers (2013), Retrieved from: http://www.mountainvalleygrowers.com/lav dentata.htm

melliferous or as aromatic plants for producing essential oil [41, 42]. Its traditional medicinal use in Arabian Peninsular region includes the treatment of wounds, rheumatism, urine retention and kidney stones. Moreover, it has also been used as antiseptic [43].

\subsubsection{Leonurus}

Leonurus L. genus comprises about 23 perennial herbs distributed particularly in Europe and Asia [44, 45]. Plants belonging to this genus are characterized by stems square in cross section, incised or toothed leaves, verticillasters of axillary flowers and fruits as angled nutlets [45].

Distinct Leonurus species are frequently consumed as tea, or used in food flavoring, including soups or beverages. Alternatively, extracts obtained from Leonurus plants are also included in food supplements [46-49]. Besides that, several species of this genus have been used as ethnopharmacological agents to fight cardiocirculatory problems such as hypertension and tachyarrhythmia. Other health properties of Leonurus plants include uterotonic, diuretic and sedative [44]. 


\subsubsection{Leonurus cardiaca $L$.}

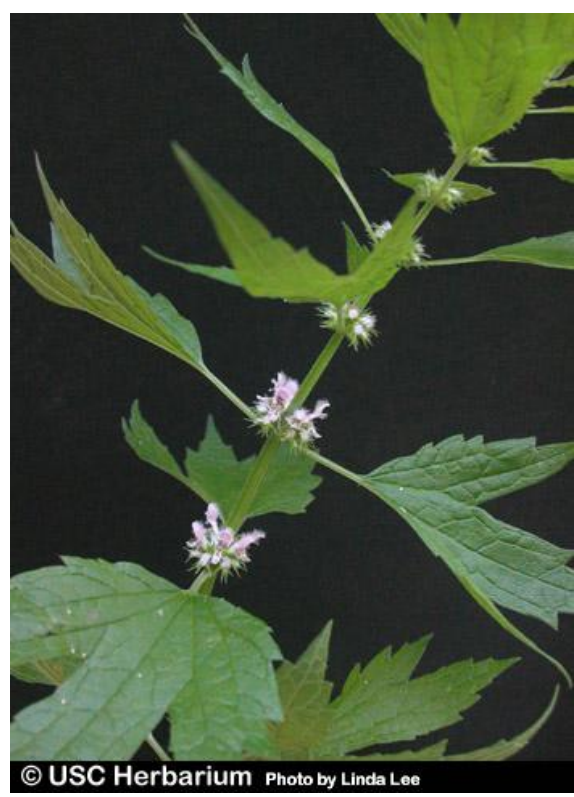

Figure 4 - Leonurus cardiaca L..

Lee, L (2013), Retrieved from: http://herbarium.biol.sc.edu/herb/LL/Le onurus_cardiaca1.jpg

Leonurus cardiaca L. (motherwort) (Fig. 4), included in the subfamily Lamioideae (Lamiaceae), is native from central Europe and it is currently spread in different temperate countries around the world. This plant grows in different types of soil and is easily found in pastures, road edges, abandoned parks, waste ground, i.e., it is globally found in rough locations $[50,51]$

Infusions of $L$. cardiaca aerial parts have been used in traditional medicine due to its claimed beneficial effects, namely sedative, uterotonic, diuretic, cardiotonic, hypotensive activities, as well as in climacteric symptoms, amenorrhea and bronchial asthma [44]. The plant is also used in homeopathic pharmacy for cardiac complaints, flatulence, and hyperthyroidism. Furthermore, because of its claimed medicinal applications, motherwort has been included in the European (2008), Russian (1968) and British Herbal (1992) Pharmacopoeias. Its aerial parts are frequently used in infusions, decoctions, syrups and tinctures, or alternatively, they are included in pharmaceutical formulations for the treatment of cardiovascular diseases [50,52]. The most described biological activities of $L$. cardiaca are the sedative, hypotensive and cardiotonic. This turns the plant a good candidate for the treatment of neuropathic and functional cardiac disorders, despite the unique indications approved by European Commission up to this moment, are those associated to nervous heart complaints and thyroid dysfunction [18, $50,53-56]$.

\subsubsection{Mentha}

Mentha L. (Lamiaceae) genus includes 30 perennial species that grow up to $120 \mathrm{~cm}$ tall, in particular in wet places. In general, these species are erect, branched, foursided or squared with growing leaves in opposite pairs and have white or purple flowers [57-60]. The plants belonging to this genus are mainly distributed in temperate regions of Europe, Asia, Australia and South Africa [61, 62]. In Mediterranean countries, some Mentha species are used as herbal teas, or alternatively, for food 
flavoring. Besides that, they have an important economic value due to their applications in food, cosmetic and pharmaceutical industries [60,63].

Since ancient times, several Mentha plants have been largely used as remedies in nasal congestion, digestive disorders and in oral hygiene. Mainly beneficial properties of Mentha plants are the anti-inflammatory, antiallergic, antipruritic, analgesic, antibacterial and anticholinesterase, which render them some applications in phytotherapeutic preparations [64-68].

\subsubsection{Mentha aquatica $L$.}

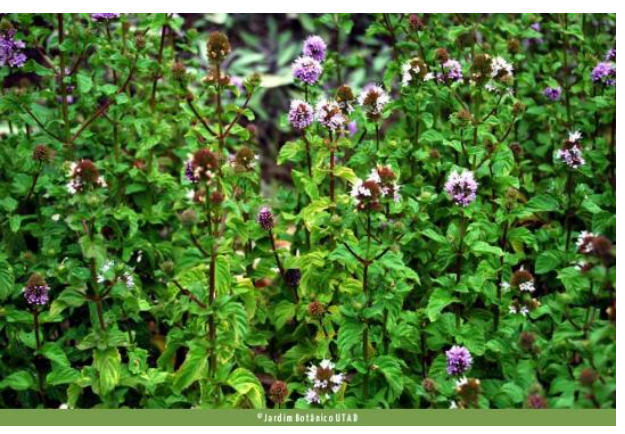

Figure 5 - Mentha aquatica L..

Jardim Botânico da UTAD (2013), Retrieved from: http://jb.utad.pt/especie/mentha_aquatica
Also known as water mint, the species Mentha aquatica L. (Fig. 5) grows in the shallow margins and channels of streams, rivers, pools, ditches and wet meadows. Their flowers are tiny, densely crowded, purple and tubular and have green or purple stems with square in cross section. The leaves are mostly green, opposite and toothed $[69,70]$.

Water mint has been used in beverages, salads or cooked foods. Some modern recipes with the plant includes "Water Mint Pesto", "Carrot and Water Mint Soup", "Warm Lamb \& Water Mint Salad", "Wild Greens with Ham" and "Orange and Mint" [71]. Besides the food applications, Mentha aquatica L. has also been consumed as tea and it has been used in traditional medicine for the treatment of external inflammation (e.g. mouth or throat problems) and in inflammation-related diseases, such as rheumatism. The plant is also frequently used as a vermifuge, in the treatment against colds and respiratory problems and to counteract mental illnesses or disorders of the central nervous system. Furthermore, it is commonly used to attenuate menstruation problems, as a stimulant and as an emetic and astringent agent [72-74].

\subsubsection{Thymus}

Thymus L. genus includes about 350 aromatic and perennial species which are distributed around the world and are particularly abundant in the west Mediterranean region, where they frequently growth in association with Lavandula, Satureja, Sideritis or Salvia plants $[75,76]$.

Pereira O. R., 2013 | CHARACTERIZATION OF PHENOLIC CONSTITUENTS OF MEDICINAL PLANTS AND EVALUATION OF PHARMACOLOGICAL ACTIVITIES: FOCUS IN ANTIOXIDANT AND ANTI-INFLAMMATORY PROPERTIES 
Thymus plants are herbaceous subshrubs or shrubs with 10 to $30 \mathrm{~cm}$ tall, containing small and simple leaves, ramified and prostrated branches and big clusters of pink, white, cream or violet flowers $[75,77]$. Thymus species are used in cosmetic and food industry and are also consumed as condiments and in the tea form, as medicinal plants $[78,79]$. The latter usage is based on their pharmacological properties, which are mainly attributed to their content in essential oils [80-83]. These beneficial properties include anti-asthmatic, bronchiolytic, expectorant, anti-septic, antimicrobial, antispasmodic, analgesic, antioxidant and anti-acetylcholinesterase activities [15, 83, 84].

\subsubsection{Thymus $x$ citriodorus}

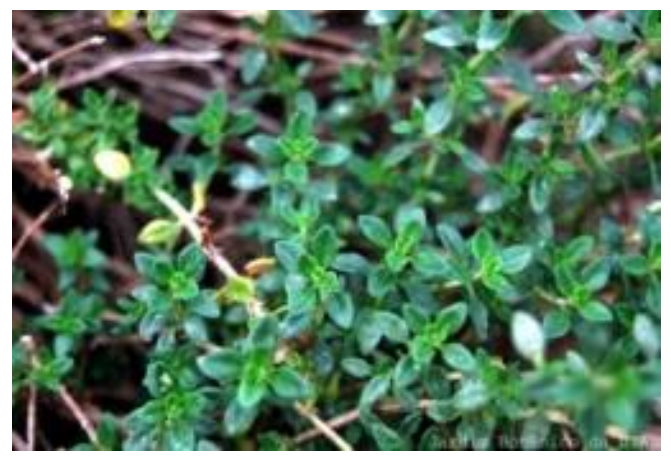

Figure 6 - Thymus $x$ citriodorus.

Jardim Botânico da UTAD (2013), Retrieved from:

http://jb.utad.pt/especie/thymus_x_citriodorus
Thymus $x$ citriodorus or lemon thyme (Fig. 6), a popular culinary herb, is a creeping plant that grows up to 8 inches high and 2 feet wide. Many short, soft, upright stems rise up from the runners and root at the nodes. The leaves are small, glossy and dark green and they have a wonderful lemony scent. In the summer, the plant produces pale lilac flowers [85]. It is used as tea, for making potpourris and in culinary for flavoring salads, fish, meat and vegetables dishes [81]. Besides theses usages, T. $x$ citriodorus is an ingredient of dermatological preparations $[86,87]$ and it is frequently used in traditional medicine as a deodorant, antiseptic, antifungal and antimicrobial element, as well as in the treatment of asthma and other respiratory diseases $[80,88]$. The essential oil of T. $x$ citriodorus is rich in geraniol (up to $60 \%$ ), geranial $(8.2 \%)$ and neral $(5.5 \%)$, being the two latter responsible for the typical lemon fragrance [80]. 


\subsection{Methods OF EXTRACTION, PURIFICATION AND CHARACTERIZATION OF PLANT PHENOLICS}

Chemically, phenolic compounds are organic compounds characterized by an aromatic ring with one or more hydroxyl groups, being synthesized in plants through the pentose phosphate, shikimate and phenylpropanoid pathways [89]. They occur in different chemical structures, including the phenolic acids, the phenylethanoid glycosides and the flavonoids [90-92], occurring from simple phenolic molecules to complex highmolecular weight polymers [89].

A revision of the main entailed techniques in phenolic compounds analysis will be described in bellow, with emphasis on the studies focusing the plant genera Cytisus, Lamium, Lavandula, Leonurus, Mentha and Thymus.

\subsubsection{Sample preparation and extraction}

As for the majority of reported works on natural products, those focusing on Cytisus, Lamium, Lavandula, Leonurus, Mentha and Thymus polyphenolics involve regular practices to allow the improvement of the resulting analytical data. Indeed, in the sample preparation step, the material has been commonly dried [93-99], lyophilized $[95,100]$ or frozen ideally at $-80^{\circ} \mathrm{C}$ [101], thus reducing the instability of polyphenols and the action of several degradative enzymes. In addition, grounding is a well establish procedure before the extraction step [62, 95, 96, 99, 102-106], as the particle size reduction of the plants increases the yield of extraction. Note that authors occasionally defatted the plant material with apolar solvents (e.g. $n$-hexane) [107-110], for prevention of high levels of lipophilic compounds in the extracts which can interfere in the polyphenols analysis. Some authors have also performed acidic or enzymatic hydrolysis in Cytisus, Mentha and Thymus plants, either before or simultaneously with the extraction procedure, when only aglycones were intended to characterize [111113].

Phenolic compounds have been mainly obtained by solvent extraction. Aqueous mixtures of methanol or ethanol are the most used ones because the majority of phenolics in the studied genera occur in their glycosidic forms. Distinct species of Cytisus, Thymus, Lavandula, Mentha, Leonurus and Lamium have been extracted with hydromethanolic solutions of $50-80 \%(\mathrm{~V} / \mathrm{V})$ to obtain phenolic acids or phenylethanoid glycosides [16, 99, 102, 105], as well as these groups combined with flavones, flavanones and flavonols $[62,100,104-106,111,114,115]$. In a similar way, 
hydroethanolic solutions of 50 to $80 \%(v / v)$ have been preferentially used for extracting phenolic acids or flavonoids in Cytisus [113], Mentha [93, 104, 105, 111, 116], Lavandula [117, 118] or Thymus [105, 119] species. Besides the above solvents, water and methanol are also frequently used to extract phenolic acids, flavonoids and phenylethanoids from several plants. Concretely, water has been used in Mentha, Thymus, Lavandula plants [107, 120-123] and methanol in Cytisus [97], Lamium [26, 124] and in different species of Leonurus plants [54, 125-127]. Acetone or aqueous acetone mixtures were previously used in Thymus and Mentha species analysis [101, 109, 128], while diethyl ether has been used in Thymus species [129-131] and ethanol solutions were used in Lamium, Lavandula and Leonurus plants [132-134].

Authors have applied different techniques in the extraction process of phenolic compounds. Stirring [16, 104, 135], homogenization using a tissue homogenizer [94, $101,106]$, maceration $[111,117,136]$ and sonication $[105,133,137-139]$ are the most frequently applied. Commonly, these techniques have been performed at room temperature $[93,94,106,124,128]$, in order to minimize the structural degradation of phenolic compounds [140]. The extraction by means Soxhlet apparatus [54, 97] and water extraction are the main exceptions, since authors frequently have applied boiling or refluxing solvents $[107,120,121]$.

\subsubsection{Clean-up and fractionation}

The main extraction process can be followed by additional purification of the enriched phenolic extracts. This practice allows obtaining a cleaner sample for characterization or to be used in biological assays. Reported studies focusing on the genera herein studied have applied liquid-liquid extraction [125, 141] and, most commonly, solid phase extraction on $\mathrm{C}_{18}$ cartridges or column chromatography on Sephadex $\mathrm{LH}-20$. The two latter usually enclose sequential solubilisation with distinct solvents, according to the nature of compounds that are intended to separate $[54,102,107,108,119,120$, $128,131,136,142,143]$.

\subsubsection{Detection and characterization of phenolic compounds}

From the distinct methods used to estimate the total polyphenols in plant tissues, the Folin Ciocalteu [144] is the most popular and it is considered a valid approach despite its limitations [145, 146]. Still, HPLC is the technique of choice in the analysis of plant phenolics, since it allows a rapid qualitative and individual quantitative screening [143]. 
The HPLC analysis of the Cytisus, Mentha, Thymus, Lavandula, and Lamium plant extracts have been essentially carried out on $C_{18}$ reversed-phase columns $[26,111$, $138,147]$. Additionally, in order to control the reproducibility of the method, the column temperature is usually maintained constant $\left(20-35^{\circ} \mathrm{C}\right)[16,97]$.

Other important feature for achieving a good separation of phenolic constituents and consequently, high accuracy in the method, is the choice of the mobile phase. Distinct combinations of mobile and stationary phases provide different compound separation, since this is based on the polarity differences among phenolic compounds [148]. In species belonging to Cytisus, Mentha, Thymus, Lavandula, and Lamium genera, phenolic compounds have been preferentially analyzed in a binary system of solvents, that consist in mixtures of acetonitrile/water [16, 93, 97, 104, 120, 149] or methanol/water $[111,113,134,135,138]$. Some works focusing in the target plant genus applied acetonitrile/water or methanol/water combinations for fractionation of phenolic compounds $[123,134,138,150]$. Note that acidified water $(0.1 \%$ to $5 \%$ of formic acid, acetic acid or less commonly phosphoric acid) is preferentially used, as this procedure impairs analytes ionization and thus allows a better resolution and superior reproducibility of the retention times, as well as the minimization of peak tailing $[143,148,151]$.

As commonly, the HPLC separation of phenolic compounds in the target genera has been achieved at constant flow rates of approximately $1 \mathrm{~mL} / \mathrm{min}$ and their identification and quantification has been frequently done by comparison of the retention times and integrated peak areas of the separated compounds, to those of the corresponding reference compound $[26,94,95,101,120,134,138,147,149,152]$. This information has also been combined to spectral information gathered by photodiode array detector (PDA) [61, 62, 94, 95, 111, 114, 129, 130, 153]. Spectral data in those studies has been obtained in the range of 200 to $450 \mathrm{~nm}$, while the chromatograms of phenolics compounds have been plotted according to their maximum absorbance peaks: at 280 $\mathrm{nm}$ for flavanones and hydroxybenzoic acids, at 320-330 nm for hydroxycinnamic acids and flavones and at 350-370 nm for flavonols [16, 95, 96, 101, 111, 123]. Alternatively, in case of exclusive usage of UV-Vis detector, the polyphenolic profiles are only recorded at a wavelength of $280 \mathrm{~nm}[97,102,103,112,154,155]$.

Due to commercial unavailability of many phenolic plant constituents, fine analytical techniques have also been implemented in order to improve the phenolic characterization. In this field, mass spectrometry has been playing a crucial role, as its coupling to chromatographic analysis allowed an increment on the sensitivity and selectivity of the method. HPLC fractionation combined with electrospray ionization- 
MS/MS analyses have been used e.g. by Krzyzanowska and colleagues [62], for structural determination of phenolic acids, flavones and flavanones in two species of Mentha $[62,156]$. Since these procedures entail a long time of analysis, the present implementation of faster and reliable analytical methodologies, as e.g. the chromatographic techniques hyphenated with mass spectrometry appears as a good alternative. On-line LC-MS/MS analysis has been used in the identification of phenolic acids and distinct classes of flavonoids in M. piperita [116], as well as in several Thymus species [106, 114, 129, 135], Cytisus [16, 138] and Lavandula [149, 157] plants. In the majority of these studies, mass spectrometry analysis has been performed using electrospray ionization (ESI), a soft mode of ionization that is suitable for structural characterization of a high number of polar biomolecules, including the phenolic compounds. Moreover, the mass spectrometry analysis has been mainly carried out in the negative ion mode, due to its high sensitivity in detecting distinct classes of phenolic compounds [158].

Additionally, sometimes coupled with techniques as LC or MS, nuclear magnetic resonance (NMR) spectroscopy has been used to achieve the exact structure of isolated phenolic compounds from several species of the Leonurus [125, 126, 159], Lamium [124, 132, 160], Thymus [119], Lavandula [117, 161] and Mentha [107, 162]. As known, NMR is a powerful technique for structural characterization. Its main drawback is its low sensibility when compared to MS and thus, there is the need of getting higher amounts of sample for analysis [163]. In this sense, when using NMR technique, samples need to be obtained by preparative chromatography [107, 119, 132, 164, 165]. A good alternative is the coupling of HPLC with NMR techniques (LCNMR) that actually appears as the most powerful method for the separation and structural determination of organic compounds. Regardless of its efficiency for identification of on the nature of the polyphenol skeletons and on their substitution patterns, the method is not widely used at present due the high entailed costs [148]. 


\subsection{Phenolic COMPounds In the taRget PLANT GenUS}

A detailed overview on the phenolic compounds of Cytisus, Lamium, Lavandula, Leonurus, Mentha and Thymus genera is described in this section. Data is summarized in Tables 1, 2, 3, 4, 5 and 6 and the structure of each polyphenol is depicted in Fig. 8, $10,13,15,17$ and 19.

\subsubsection{Phenolic acids}

Phenolic acids are compounds characterized by a phenolic ring with an organic carboxylic acid function and can occur as hydroxybenzoic (C6-C1) (Fig. 7A) and hydroxycinnamic (C6-C3) acids (Fig. 7B). Following, a review of the phenolic acids from Cytisus, Lamium, Lavandula, Leonurus, Mentha and Thymus genera is described. Data is summarized in Table 1 and the structure of each compound is depicted in Fig. 8.<smiles>O=C(O)c1ccc(O)cc1</smiles>

A<smiles>O=C(O)/C=C/c1ccc(O)cc1</smiles>

B

Figure 7 - General structure of hydroxybenzoic (A) and hydroxycinnamic (B) acids.

Phenolic acids are largely described in Mentha, Thymus and Lavandula species being the most reported the hydroxycinnamic caffeic acid and its dimer rosmarinic acid. Despite caffeic acid (1) has been reported in a high number of Mentha species (Table 1 ), this is only present in minor amounts, contrasting with rosmarinic acid (2), which is described to vary between 3.1 to $19.1 \mathrm{mg} / \mathrm{g}$ of dry plant in $M$. x piperita, M. aquatica, $M$. $x$ dalmatica, and $M$. canadensis plants. These amounts represent at least $30 \%$ of the total phenolic compounds quantified in those plants $[61,95,102,111,120,152,166]$. In a similar way, caffeic acid has been largely reported in Thymus species (see Table $1)$, regardless it only occurs in low amounts $(0.1-0.48 \mathrm{mg} / \mathrm{g}$ of dry plant) [94, 121]. Instead, rosmarinic acid has been described has a major phenolic compound in several Thymus species (Table 1), representing about $70 \%$ of the total polyphenols quantified in $T$. vulgaris extracts [94, 95, 154]. This caffeic acid dimer is a major phenolic constituent of several Lavandula species with amounts ranging from 0.1 to $11.1 \mathrm{mg} / \mathrm{g}$ of dry plants [123, 134, 157, 167-169]. 
Besides rosmarinic acid, Mentha, Thymus and Lavandula species have been described to contain other caffeic acid derivatives. In particular, $T$. vulgaris extracts were reported to have rosmarinic acid glucoside (3), 3'-O-(8'-Z-caffeoyl)rosmarinic acid (4), rosmarinic acid methylester $(0.6 \mathrm{mg} / \mathrm{g}$ of dry plant) (5), salvianolic acid $\mathrm{I}(6)$ and salvianolic acid K (7). Lithospermic acid (8) was described to account for $12 \mathrm{mg} / \mathrm{g}$ of dry T. serpyllum plant [154]. Other caffeic acid derivatives in Thymus genus include the glucoside of caffeic acid (9), caffeic acid ethyl ester (10) and dicaffeoylquinic (11) or chlorogenic acids (12) [106, 114, 135, 153]. The latter compound has been reported in M. x piperita [111] while nepetoidin A (13) and nepetoidin B (14), lithospermic acid, lithospermic acid $B$ (15), salvianolic acid $J(16)$ and salvianolic acid $L$ (17), between others (18-21), have been described in several Mentha species [120, 128, 164], as summarized in Table 1. Furthermore, several Lavandula species have been described to contain caffeic acid derivatives, namely the dicaffeoylquinic acid [157], the 3-O $(0.7$ $\mathrm{mg} / \mathrm{g}$ of dry plant), $4-\mathrm{O}$ (22) $(0.5 \mathrm{mg} / \mathrm{g}$ of dry plant) and $5-\mathrm{O}$ caffeoylquinic acids (23) (0.2 mg/g of dry plant) [168] (Table 1$)$.

Hydroxycinnamic acids unrelated with caffeic acid plus hydroxybenzoic acids as gallic (24), gentisic (25), syringic acid (26), ferulic (27), ferulic-O-glucoside (28), sinapic (29) and protocatechuic acids (30) have been also reported in several Thymus and/or Lavandula species $[102,123,149,170]$.

Regarding the three remaining plant genera in focus in the present study (Lamium, Leonurus and Cytisus), we should remark that they are much poor in phenolic acids, as compared to the previous described ones. Indeed, to the best the author's knowledge, this class of compounds has not been detected yet in Cytisus plants. Moreover, only syringic acid and p-hydroxybenzoic acid (31) were reported for Leonurus plants, in particular for L. sibiricus extracts [127], while caffeic acid, chlorogenic acid, protocatechuic acid , p-coumaric acid (32), ferulic acid and vanillic acid (33) have been described in Lamium album extracts $[17,26,171]$. 
Table 1 - Phenolic acids of Cytisus, Lamium, Lavandula, Leonurus, Mentha and Thymus genera.

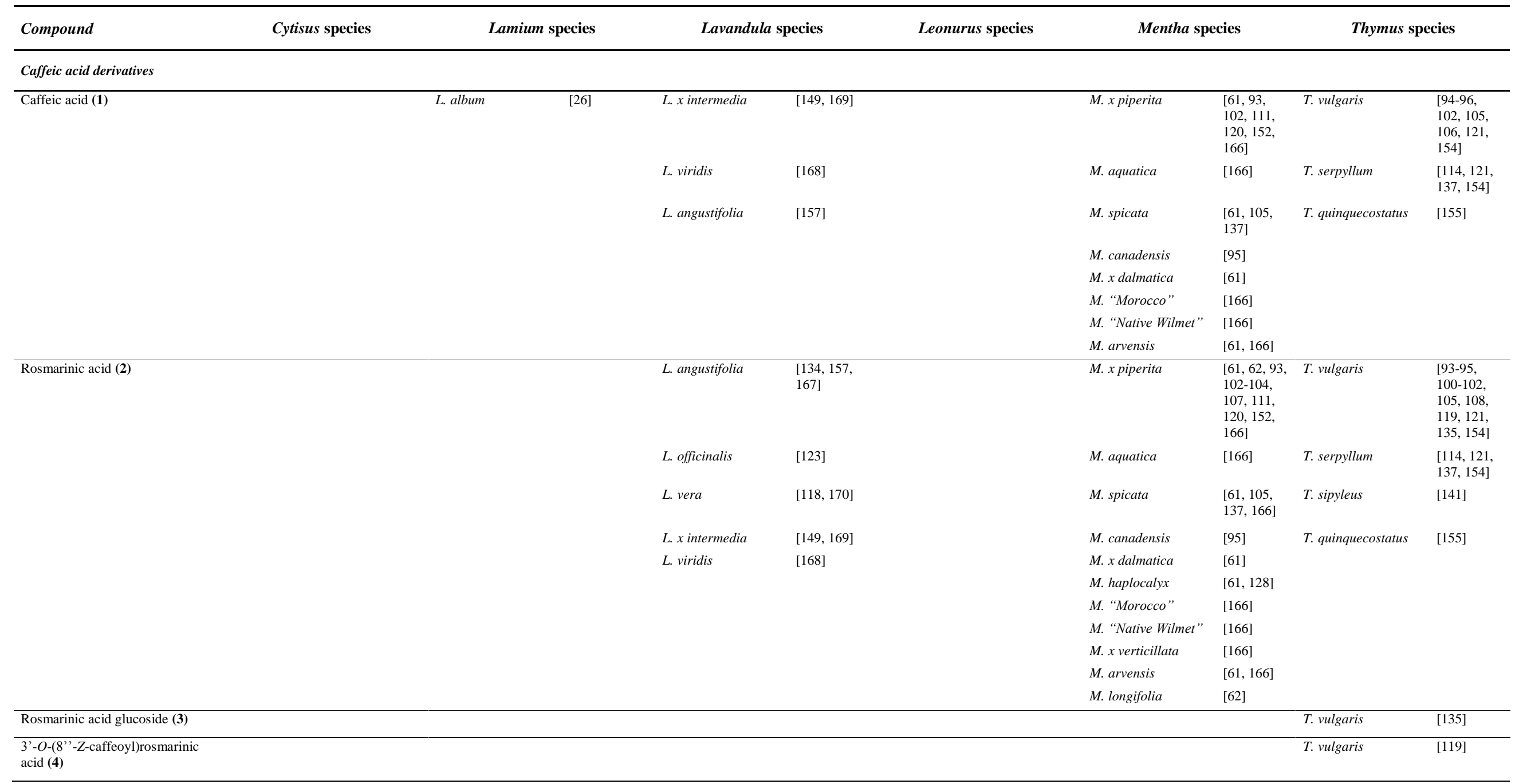




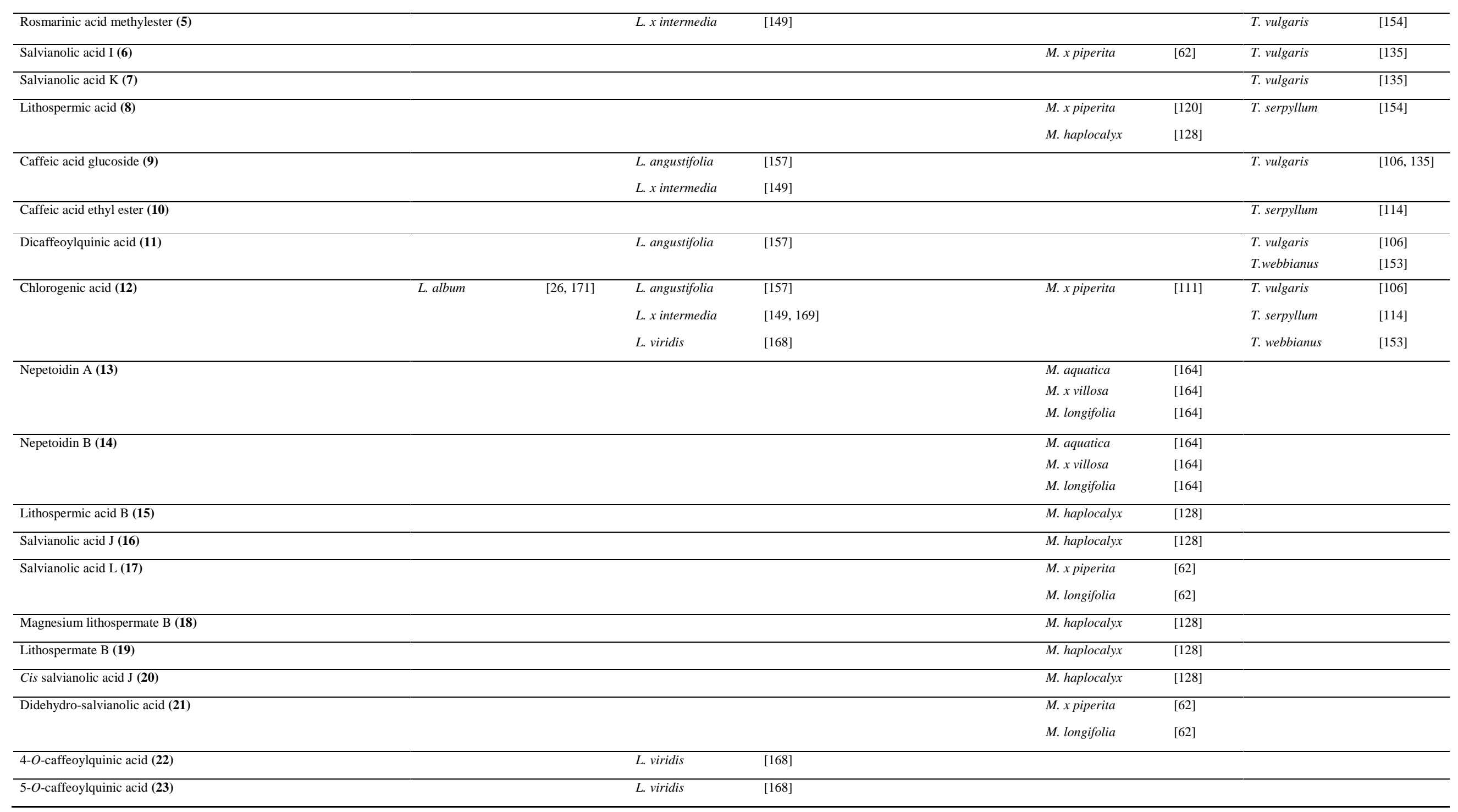




\section{0 | INTRODUCTION}

\begin{tabular}{|c|c|c|c|c|c|c|c|c|c|c|}
\hline \multicolumn{11}{|l|}{ Other phenolic acids } \\
\hline Gallic acid (24) & & & & & & & & & T. vulgaris & $\begin{array}{l}{[94,95,} \\
102,106]\end{array}$ \\
\hline Gentisic acid (25) & & & & & & & M. x piperita & {$[102]$} & T. vulgaris & {$[102]$} \\
\hline \multirow[t]{2}{*}{ Syringic acid (26) } & & & & & L. sibiricus & [127] & M. x piperita & [102] & T. vulgaris & $\begin{array}{l}{[102,106,} \\
121]\end{array}$ \\
\hline & & & & & & & & & T. serpyllum & [114] \\
\hline \multirow[t]{3}{*}{ Ferulic acid (27) } & L. album & [26] & L. angustifolia & [157] & & & & & T. vulgaris & {$[96,106]$} \\
\hline & & & L. officinalis & [123] & & & & & & \\
\hline & & & L. x intermedia & [169] & & & & & & \\
\hline \multirow[t]{2}{*}{ Ferulic acid- $O$-glucoside (28) } & & & L. angustifolia & {$[157]$} & & & & & & \\
\hline & & & L. $x$ intermedia & [149] & & & & & & \\
\hline Sinapic acid (29) & & & L. vera & [170] & & & & & & \\
\hline \multirow[t]{3}{*}{ Protocatechuic acid (30) } & L. album & [26] & L. $x$ intermedia & [149] & & & M. x piperita & {$[102]$} & T. vulgaris & {$[102,106]$} \\
\hline & & & & & & & & & T. quinquecostatus & [155] \\
\hline & & & & & & & & & T. webbianus & [153] \\
\hline \multirow[t]{3}{*}{ p- hydroxybenzoic acid (31) } & & & & & L. sibiricus & [127] & & & T. serpyllum & [114] \\
\hline & & & & & & & & & T.webbianus & [153] \\
\hline & & & & & & & & & T. serpyllum & [114] \\
\hline \multirow[t]{3}{*}{$p$-coumaric acid (32) } & L. album & [26] & & & & & & & T. vulgaris & $\begin{array}{l}{[95,102,} \\
106]\end{array}$ \\
\hline & & & & & & & & & T.webbianus & [153] \\
\hline & & & & & & & & & T. serpyllum & [114] \\
\hline \multirow[t]{2}{*}{ Vanillic acid (33) } & L. album & [26] & & & & & & & T. vulgaris & {$[102,106]$} \\
\hline & & & & & & & & & T. serpyllum & [114] \\
\hline
\end{tabular}




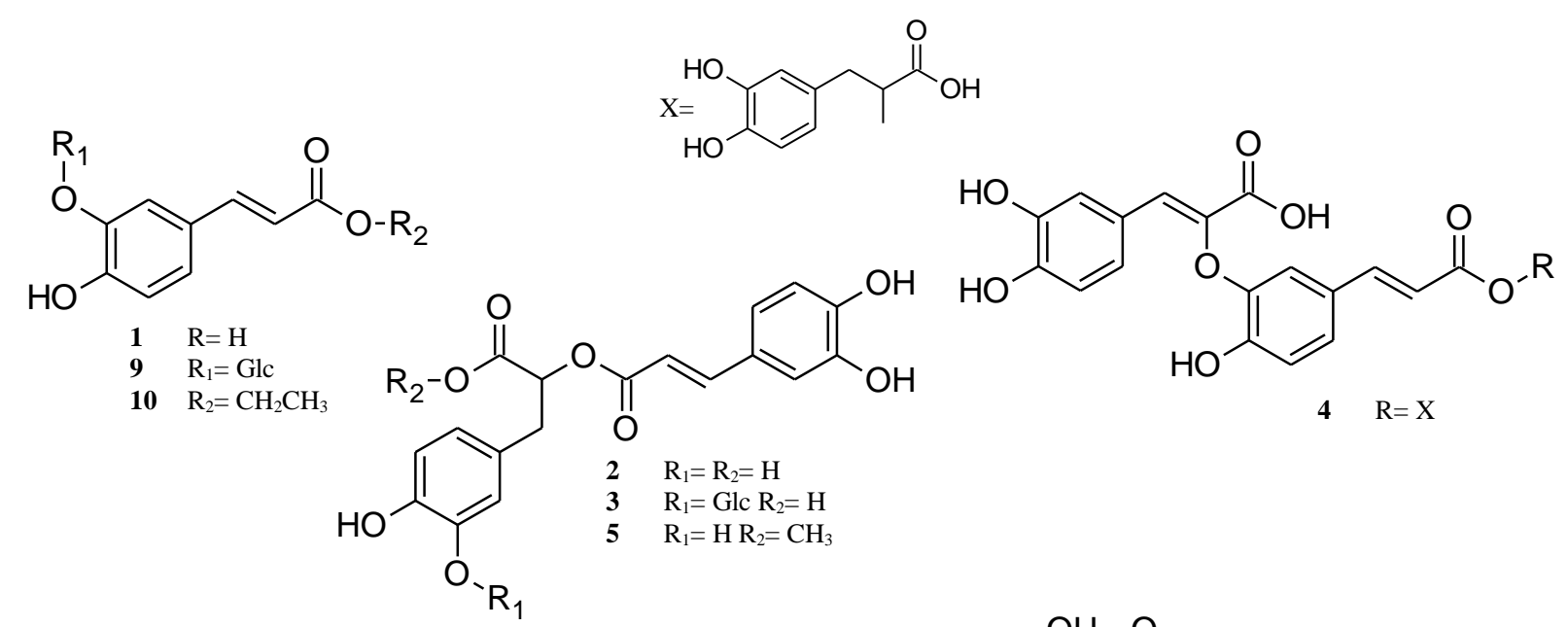<smiles></smiles><smiles>[R]OC(=O)/C=C/C1=CC=C(Oc2ccc(O)c(O)c2)[C@H](C(O)C(=O)O)[C@H]1c1ccc(O)c(O)c1</smiles><smiles>[R7]OC(=O)/C=C/c1ccc(O)c2c1C(C(=O)O[R7])[C@H](c1ccc(O)c(O)c1)O2</smiles><smiles>O=C(/C=C/c1ccc(O)c(O)c1)OC1CC(O)(C(=O)O)CC(OC(=O)/C=C/c2ccc(O)c(O)c2)C1O</smiles><smiles>[R]OC1CC(O)(C(=O)O)CC(O[R])C1O[R]([H])([H])[H]</smiles><smiles>[R2]c1cc(/C=C\OC(=O)/C=C/c2ccc(O)c(O)c2)cc(O)c1[R7]</smiles><smiles>[R]OC(=O)/C=C/c1ccc2c(c1)OC(C(=O)O)C(c1ccc(O)c(O)c1)O2</smiles><smiles>[R]OC(=O)C1=Cc2ccc(O)c(O)c2C(C(=O)O[R])C1c1ccc(O)c(O)c1</smiles> 


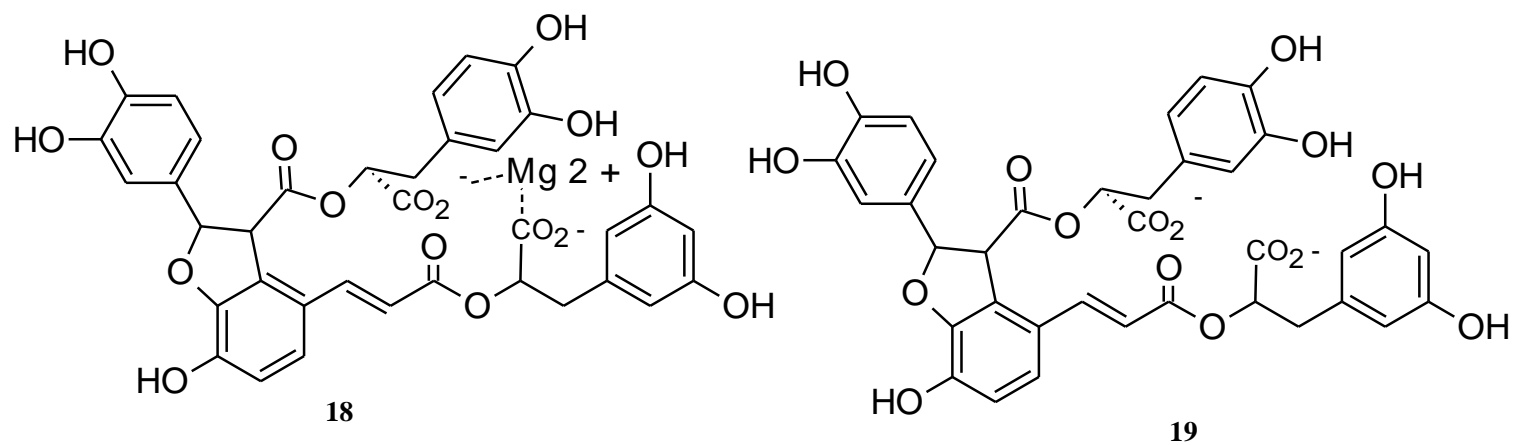<smiles>[Y][X]c1ccc2c(c1)OC(C(=O)O)C(c1ccc(O)c(O)c1)O2</smiles><smiles>[R]c1cc(C(=O)O)c([R6])c([R6])c1[R]</smiles>

$4 \mathrm{R}_{1}=\mathrm{R}_{2}=\mathrm{R}_{3}=\mathrm{OH} \mathrm{R}_{4}=\mathrm{H}$

$25 \mathrm{R}_{1}=\mathrm{R}_{4}=\mathrm{OH} \mathrm{R} \mathrm{R}_{2}=\mathrm{R}_{3}=\mathrm{H}$

$26 \mathrm{R}_{1}=\mathrm{R}_{3}=\mathrm{OCH}_{3} \mathrm{R}_{2}=\mathrm{OH} \mathrm{R} \mathrm{R}_{4}=\mathrm{H}$

$30 \mathrm{R}_{1}=\mathrm{R}_{4}=\mathrm{H} \mathrm{R}_{2}=\mathrm{R}_{3}=\mathrm{OH}$

$31 \mathrm{R}_{1}=\mathrm{R}_{3}=\mathrm{R}_{4}=\mathrm{H} \mathrm{R}_{2}=\mathrm{OH}$

$33 \mathrm{R}_{1}=\mathrm{R}_{4}=\mathrm{H} \mathrm{R}_{2}=\mathrm{OH} \mathrm{R}=\mathrm{OCH}_{3}$<smiles>[R6]c1cc(/C=C/C(=O)O)c([R5])c([R6])c1[R]</smiles>

$27 \mathrm{R}_{1}=\mathrm{OH} \mathrm{R}_{2}=\mathrm{OCH}_{3} \mathrm{R}_{3}=\mathrm{R}_{4}=\mathrm{H}$

$28 \mathrm{R}_{1}=\mathrm{OGlc} \mathrm{R}_{2}=\mathrm{OCH}_{3} \mathrm{R}_{3}=\mathrm{R}_{4}=\mathrm{H}$

$29 \mathrm{R}_{1}=\mathrm{OH} \mathrm{R}_{2}=\mathrm{R}_{4}=\mathrm{OCH}_{3} \mathrm{R}_{3}=\mathrm{H}$

$32 \mathrm{R}_{1}=\mathrm{OH} \mathrm{R}=\mathrm{R}_{3}=\mathrm{R}_{4}=\mathrm{H}$

Caff- Caffeoyl unit; Glc- Glucosyl unit

Figure 8 - Chemical structures of the phenolic acids reported in Cytisus, Lamium, Lavandula, Leonurus, Mentha and Thymus plants. 


\subsubsection{Phenylethanoid glycosides}

Phenylethanoid glycosides are a group of water soluble compounds which is widely found in plants. Structuraly, they are cinnamic acids and hydroxyphenyl ethyl moieties attached to a B-glucopyranose through ester linkages and glycosidic linkages, respectively (Fig. 9) [172]. Common hydroxycinnamic acids enclose the caffeic acid, cinnamic acid and ferulic acid, while rhamnose, apiose or arabinose are the most usual sugars found.

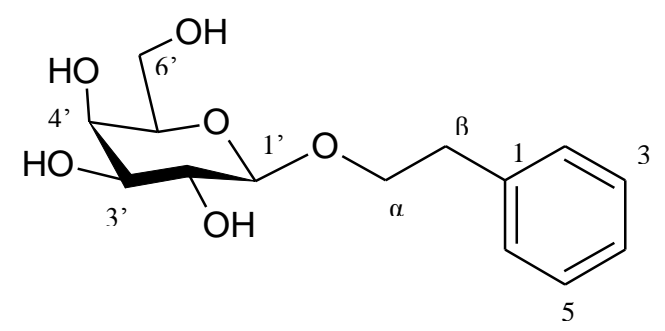

Figure 9 - General structure of phenylethanoid glycosides.

At this moment, hundreds of phenylethanoid glycoside compounds have been described in plants and some of them with proved important biological activities [90]. In between the six plant genera herein studied, phenylethanoid glycosides are reported in Lamium and Leonurus genera (Table 2, Fig. 10), while to the author's knowledge, they are absent from Mentha, Thymus, Lavandula and Cytisus plants.

In more detail, verbascoside (34) has been reported in Lamium maculatum (approximately $2 \mathrm{mg} / \mathrm{g}$ dry extract), L. album, L. garganicum and in L. purpureum [17, $124,139,173]$. This compound has also been detected in $L$. glaucescens or $L$. cardiaca extracts, from Leonurus genus [174, 175]. Moreover, the verbascoside structural isomer, the isoverbascoside (35), has been found to occur in Lamium purpureum [124], together with other less common phenylethanoid compounds (36-40) (Table 2). Also, lavandulifolioside (41) has been described in Leonurus cardiaca extracts [176] and in L. glaucescens, which also contain other compounds as leonoside A (42) and leonoside B (43) [174]. 


\section{4 | INTRODUCTION}

Table 2 - Phenylethanoid glycosides of Lamium and Leonurus genera.

\begin{tabular}{|c|c|c|c|c|}
\hline Compound & \multicolumn{2}{|c|}{ Lamium species } & \multicolumn{2}{|c|}{ Leonurus species } \\
\hline \multicolumn{5}{|l|}{ Phenylethanoid glycosides } \\
\hline \multirow[t]{6}{*}{ Verbascoside (34) } & L. album & [17] & L. glaucescens & {$[$ [174] } \\
\hline & L. garganicum & [17] & L. cardiaca & [175] \\
\hline & L. album & [17] & & \\
\hline & L. maculatum & {$[139,173]$} & & \\
\hline & L. maculatum & {$[173]$} & & \\
\hline & L. purpureum & [124] & & \\
\hline Isoverbascoside (35) & L. purpureum & [124] & & \\
\hline \multirow[t]{2}{*}{ Lamalboside (36) } & L. purpureum & {$[17]$} & & \\
\hline & L. album & {$[171]$} & & \\
\hline Lamiuside C (37) & L. purpureum & {$[124]$} & & \\
\hline Lamiuside D (38) & L. purpureum & [124] & & \\
\hline Campneoside I (39) & L. purpureum & [124] & & \\
\hline Leucosceptoside A (40) & L. purpureum & {$[124]$} & & \\
\hline \multirow[t]{2}{*}{ Lavandulifolioside (41) } & & & L. cardiaca & [176] \\
\hline & & & L. glaucescens & [174] \\
\hline Leonoside A (42) & & & L. glaucescens & [174] \\
\hline Leonoside B (43) & & & L. glaucescens & [174] \\
\hline
\end{tabular}

$34 \mathrm{R}_{1}=\mathrm{R}_{2}=\mathrm{R}_{3}=\mathrm{R}_{4}=\mathrm{H}$

$36 \quad \mathrm{R}_{1}=\mathrm{R}_{2}=\mathrm{R}_{4}=\mathrm{H} \mathrm{R}_{3}=\mathrm{Ga}$

$38 \mathrm{R}_{1}=\mathrm{OCH}_{3} \mathrm{R}_{2}=\mathrm{R}_{4}=\mathrm{H} \mathrm{R}_{3}=\mathrm{Ga}$

$39 \mathrm{R}_{1}=\mathrm{OCH}_{3} \mathrm{R}_{2}=\mathrm{R}_{3}=\mathrm{R}_{4}=\mathrm{H}$

$40 \quad \mathrm{R}_{1}=\mathrm{R}_{3}=\mathrm{R}_{4}=\mathrm{H} \mathrm{R}_{2}=\mathrm{CH}_{3}$

$41 \quad \mathrm{R}_{1}=\mathrm{R}_{2}=\mathrm{R}_{4}=\mathrm{H} \mathrm{R}_{3}=$ Ara

$42 \mathrm{R}_{1}=\mathrm{R}_{4}=\mathrm{H} \mathrm{R}_{2}=\mathrm{CH}_{3} \mathrm{R}_{3}=$ Ara

$43 \quad \mathrm{R}_{1}=\mathrm{H} \mathrm{R}_{2}=\mathrm{R}_{4}=\mathrm{CH}_{3} \mathrm{R}_{3}=$ Ara

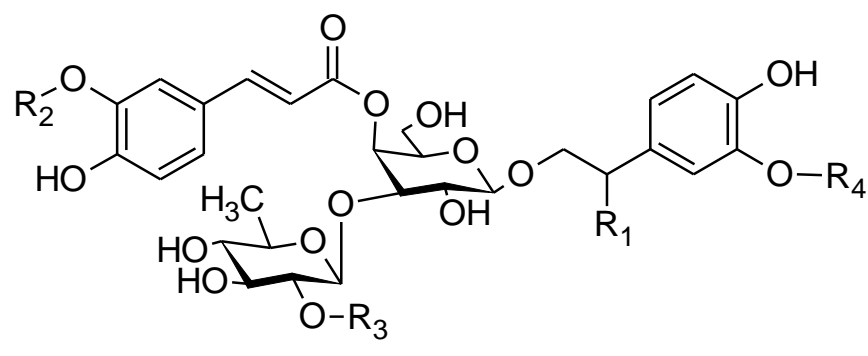

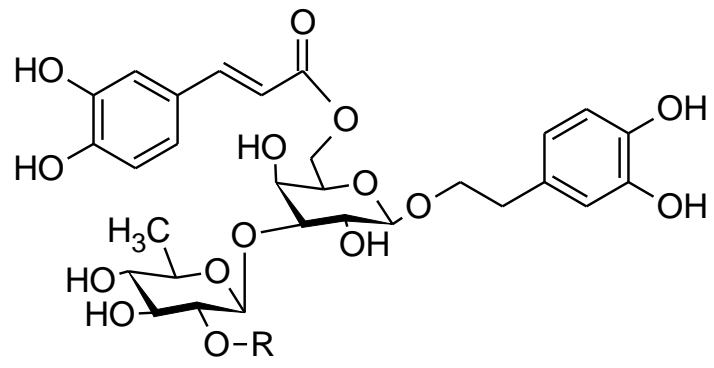

Ara- Arabinosyl unit; Gal- Galactosyl unit

Figure 10 - Chemical structures of phenylethanoid glycosides reported in Lamium and Leonurus plants. 


\subsubsection{Flavonoids}

Flavonoids are molecules characterized by a C15 structure (C6-C3-C6) with a heterocyclic benzopyran ring ( $C$ ring), an aromatic ring ( $A$ ring) and a phenyl constituent as the B ring (Fig. 11), all of them with several structural variations. Flavonoids are divided in six main distinct classes (flavones, isoflavones, flavonols, flavanones, flavanols and anthocyanidins), according to their oxidation state, the connection of an aromatic ring and the functional groups of the $\mathrm{C}$ ring. Up to this moment, more than 4000 flavonoids have been identified in plants, mainly occurring as glycosides [91].

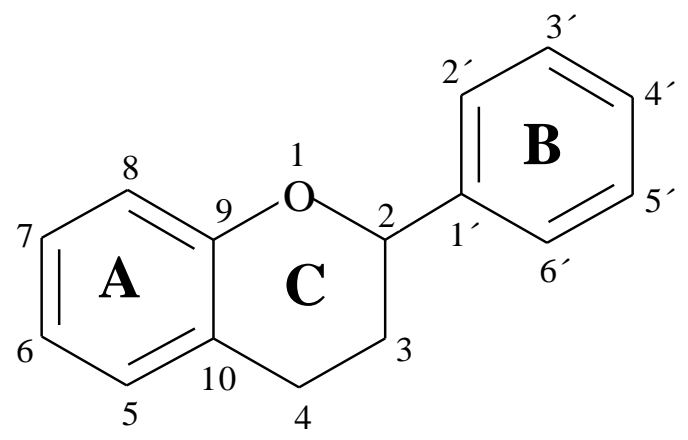

Figure 11 - General structure of flavonoids.

Overall, plants of the target genera are enriched in distinct classes of flavonoids, with particular emphasis on mono or di-O and $C$-glycosidic derivatives of the flavones luteolin and apigenin, of the flavanones eriodictyol and naringenin or of the flavonol quercetin.

\subsubsection{Flavones}

Flavones comprises a class of flavonoids characterized by the presence of a double bond between 2 and 3 position, containing several A- and B-rings substitutions and lacking oxygenation at the 3-position of the C-ring (Fig. 12). 


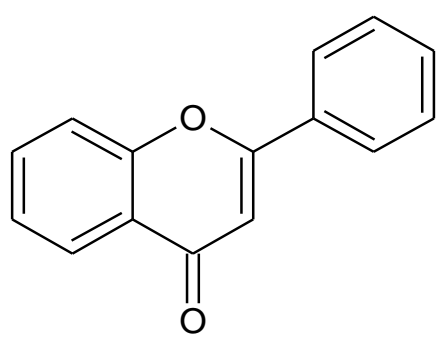

Figure $12-$ General structure of flavones.

For the six plant species in focus, flavones are mainly found in Mentha, Thymus and Lavandula plants, with the prevalence of luteolin and apigenin glycosidic derivatives, as described in bellow.

Luteolin-7-O-glucoside (44) has been detected in several Lavandula species [30, 117, 177-179]. Its amounts in L. viridis extracts [168] have been reported to be of $3.8 \mathrm{mg} / \mathrm{g}$ of dry plant. The same compound is also largely reported in Mentha plants (Table 3, Fig. 13), accounting from 0.1 to $3 \mathrm{mg} / \mathrm{g}$ of dry plant in species as $M . x$ piperita, $M$. aquatica and $M$. arvensis $[61,104,166]$. Concerning the Thymus species, the luteolinO-derivatives have been detected in $T$. vulgaris, $T$. serpyllum, $T$. sipyleus and $T$. webbianus [114, 121, 153] while luteolin-acetyl-O-glycoside (45), luteolin-O-diglucoside (46) have been reported in T. vulgaris and luteolin-7-O-(6"-feruloyl)-ß-glucopyranoside (47) has been described to occur in T. sipyleus [100, 141].

Note that besides glucose, other sugar forms such as glucuronic acid and rutinose are also frequently linked to the flavone skeleton on Mentha, Thymus and Lavandula plants [158]. In summary, O-glucuronide derivatives of luteolin have been detected in L. $x$ intermedia, L. stoechas and L. dentata [30, 149]. Significant amounts of luteolin-7-Oglucuronide (48) have been reported in T. vulgaris and T. serpyllum plants (8 and 14 $\mathrm{mg} / \mathrm{g}$ of dry plant, respectively) [154]. Moreover, luteolin-O-glucuronide compounds together with luteolin-O-glucuronide-methyl (49) and luteolin-O-diglucuronide (50), have been described for M. x piperita and $M$. longifolia species [62, 93, 120].

Regarding the 7-O-rutinoside derivative of luteolin (51), this has been vastly described to occur in $M$. x piperita $[93,103,104,107,120]$ with a concentration of $8 \mathrm{mg} / \mathrm{g}$ of dry M. $x$ piperita. Lower concentrations (about $1.4 \mathrm{mg} / \mathrm{g}$ of dry plant) were found to occur in Thymus plants [106, 154]. To the author's knowledge, this compound was not quantified in Lavandula plants, regardless its presence has been confirmed in $L$. dentata [178]. Besides the above luteolin O-sugar derivatives, it should also be 
mentioned that 2"-O-pentosyl-8-C-hexoside luteolin (52) has been described for $L$. angustifolia extracts [157].

Contrarily to the above luteolin derivatives, the aglycone luteolin (53) has been much less reported in Mentha and Thymus genera, and mostly detected as a minor phenolic component. It has been detected in L. dentata [178, 179], L. stoechas [30] and $L$. angustifolia (0.02 mg/g of dry plant) extracts [134]. In M. arvensis, M. haplocalyx and M. spicata species, its concentration was reported to be up to $0.12 \mathrm{mg} / \mathrm{g}$ of dry plant $[61,93,111,180,181]$ while its amounts in $T$. vulgaris and $T$. serpyllum plants varies between 0.6 to $1.5 \mathrm{mg} / \mathrm{g}$ of respectively [154].

As described above, luteolin derivatives are not widespread in Cytisus, Lamium and Leonurus genus. To the author's knowledge, 2"-O-pentoxide-8- $C$-hexoside luteolin, luteolin-7-O-glucoside and luteolin-7-methylether (54) are the only reported phenolics for each of these genera, respectively $[16,127,160]$.

Apigenin glycosides are frequently found in some Lavandula, Mentha, Thymus and Leonurus plants. Concretely, and as shown in Table 3, apigenin-7-O-glucoside (55) has been reported in several Lavandula species [30, 117, 149, 177-179] with a content of about $0.04 \mathrm{mg} / \mathrm{g}$ of dry in L. angustifolia [134]. More, three O-glucoside structural isomers have been described in L. cardiaca extracts $(56,57)$ [54] (Table 3), while the most widespread isomer (apigenin-7-O-glucoside) was detected in the T. webbianus, T. vulgaris and T. serpyllum plant species [94, 106, 121, 153]. To the author's knowledge, the only apigenin glycoside described in Cytisus up to this moment is a diglycosidic derivative of this flavone (58) [16].

Besides the O-glucoside derivatives of apigenin, other sugar derivatives of this aglycone have been already described for Lavandula, Mentha and Thymus. Namely, apigenin-7-O-glucuronide (59) has been described for T. webbianus, T. vulgaris and $L$. dentata [30, 93, 100, 114, 135], while apigenin-7-O-rutinoside (60) has been reported in $L$. angustifolia and $L$. dentata plants $[157,178]$ and it is largely spread in Mentha plants $[61,93,100,103,104,107,120,166]$.

Albeit less frequent, apigenin- $C$-glycosides have also been described in Lavandula, Mentha and Thymus plants. Apigenin-8-C-glucoside (61) has been detected in $L$. angustifolia [157] and $L$. dentata [30, 178, 179] while the 6,8-di- $C$-glucoside derivative (62) was reported in the former species $[93,178,179]$ and also in $T$. webbianus [153]

The apigenin aglycone (63) has been detected in several Lavandula species [30, 149, 178, 179] (Table 3). Its content in L. angustifolia was of $0.04 \mathrm{mg} / \mathrm{g}$ of dry plant [134]. Apigenin has been also found in Leonurus plants, namely in $L$. cardiaca and in $L$. 
sibiricus $[127,133]$. Note also that this flavone has been exhaustively described for Thymus and Mentha species (Table 3). Quantification data indicate concentrations of 0.01 and $0.03 \mathrm{mg} / \mathrm{g}$ of dry plant in $M$. spicata and $M$. arvensis species, respectively [61, 166].

Besides luteolin and apigenin derivatives, other flavones should also be highlighted. Indeed, chrysin O-glycosidic derivatives seem to be the most representative phenolics of $C$. multiflorus, accounting for more than half of its total polyphenols (35.1 mg/g of dry flowers) [16]. Also, isoscutellarein $(64)$ and hypolaetin $(65,66)$ glycosides have been reported in Lavandula coronopifolia and L. pubescens extracts [161], while derivatives of the methylated chrysoeriol $(67,68)$ have been described in L. intermedia [149] and the genkwanin aglycone (69) has been identified in $L$. dentata plus in distinct Leonurus species [126, 182-185]. Moreover, the 6"-O acetyl derivative of the methoxyflavone scoparin (70) has been described to occur in C. scoparius [4]. Other less common aglycones derived from methylated flavones (71-82) have been found in several Mentha $[104,162,181]$ and Thymus $[131,186,187]$ species. 
Table 3 - Flavones of Cytisus, Lamium, Lavandula, Leonurus, Mentha and Thymus genera.

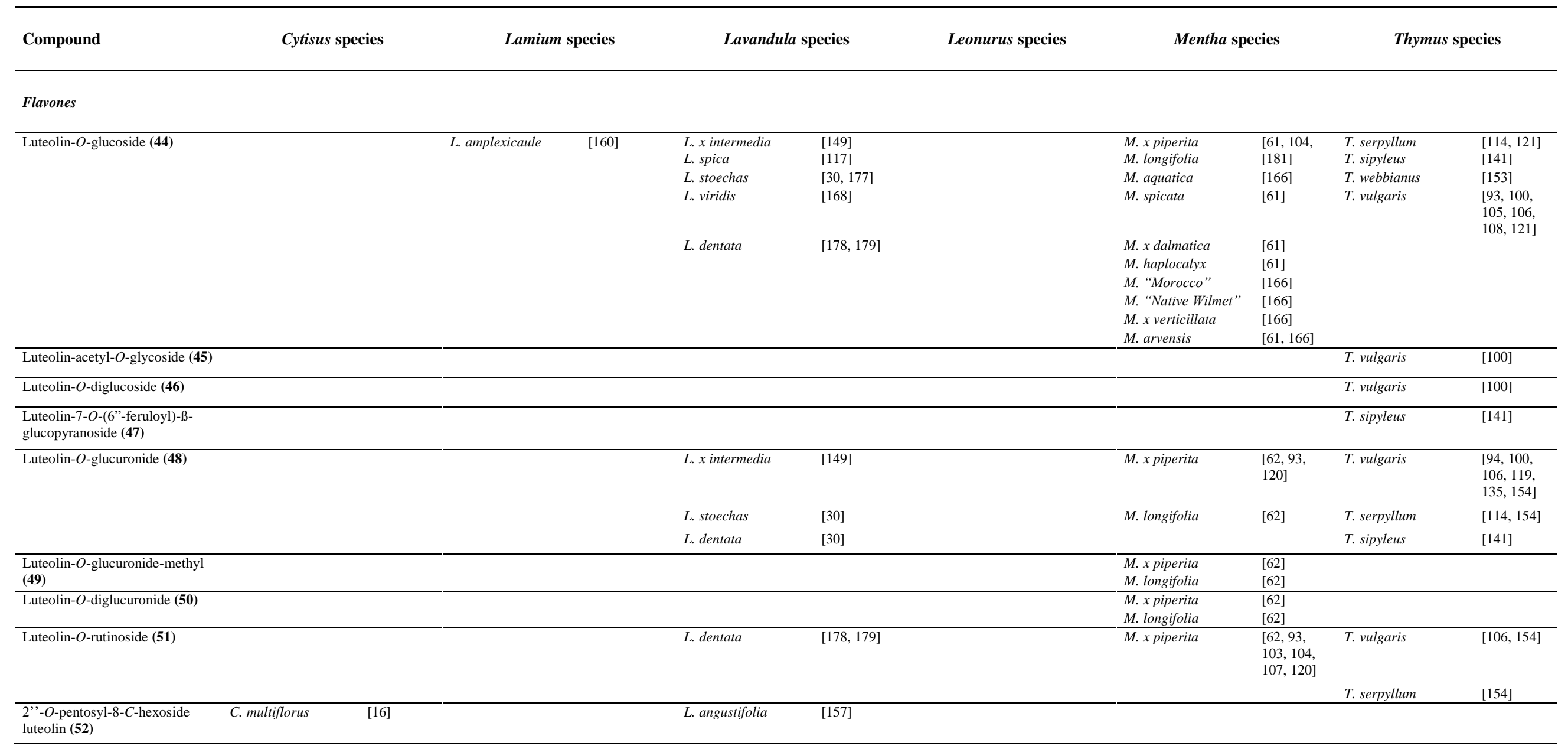




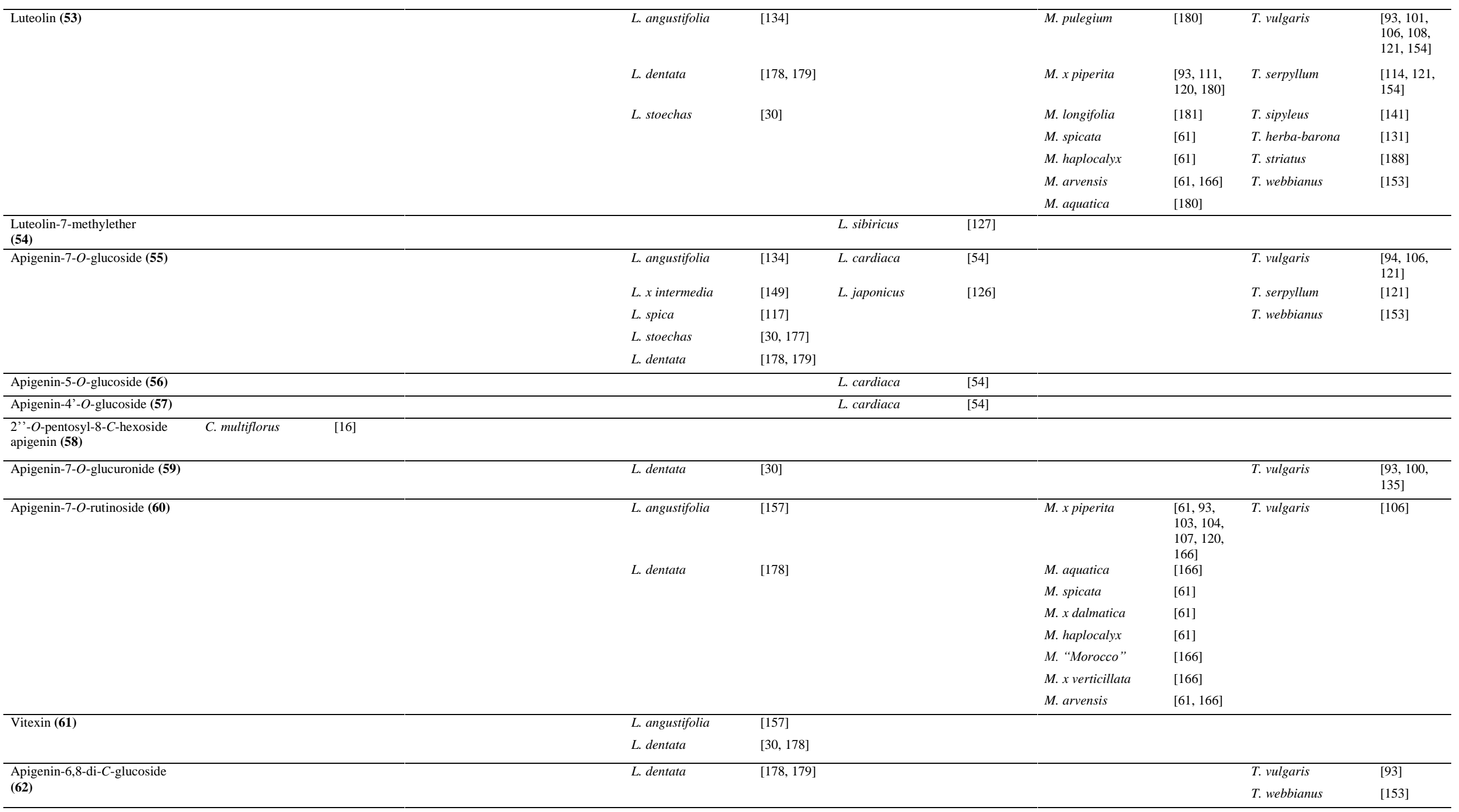




\begin{tabular}{|c|c|c|c|c|c|c|c|c|}
\hline \multirow[t]{6}{*}{ Apigenin (63) } & L. angustifolia & [134] & L. cardiaca & [133] & M. x piperita & [111] & T. vulgaris & $\begin{array}{l}{[106,108,} \\
121]\end{array}$ \\
\hline & L. dentata & $\begin{array}{l}{[30,178,} \\
179]\end{array}$ & L. sibiricus & [127] & M. spicata & [61] & T. serpyllum & {$[114,121]$} \\
\hline & L. $x$ intermedia & [149] & & & M. pulegium & [180] & T. herba-barona & [131] \\
\hline & L. stoechas & [30] & & & M. arvensis & {$[61,166]$} & T. striatus & [188] \\
\hline & & & & & M. aquatica & [180] & T.webbianus & [153] \\
\hline & & & & & M. x piperita & [180] & & \\
\hline \multirow{2}{*}{$\begin{array}{l}\text { Isoscutellarein-8-O-glucuronide } \\
\text { (64) }\end{array}$} & L. coronopifolia & [161] & & & & & & \\
\hline & L. pubescens & [161] & & & & & & \\
\hline \multirow[t]{2}{*}{ Hypolaetin-8-O-glucuronide (65) } & L. coronopifolia & [161] & & & & & & \\
\hline & L. pubescens & [161] & & & & & & \\
\hline \multirow{2}{*}{$\begin{array}{l}\text { Hypolaetin-4'-methyl ether 8-O- } \\
\text { glucuronide (66) }\end{array}$} & L. coronopifolia & {$[161]$} & & & & & & \\
\hline & L. pubescens & {$[161]$} & & & & & & \\
\hline Chrysoeriol-O-hexoside (67) & L. $x$ intermedia & [149] & & & & & & \\
\hline Chrysoeriol- $O$-glucuronide (68) & L. $x$ intermedia & [149] & & & & & & \\
\hline \multirow[t]{4}{*}{ Genkwanin (69) } & L. dentata & $\begin{array}{l}{[30,178,} \\
179]\end{array}$ & L. heterophyllus & [184] & & & & \\
\hline & & & L. sibiricus & {$[182,183]$} & & & & \\
\hline & & & L. persicus & [185] & & & & \\
\hline & & & L. japonicus & [126] & & & & \\
\hline 6"-O-acetyl scoparin (70) & [4] & & & & & & & \\
\hline \multirow[t]{2}{*}{ Thymusin (71) } & & & & & M. spicata & [162] & T. herba-barona & [131] \\
\hline & & & & & M. x piperita & {$[162,180]$} & T. striatus & [188] \\
\hline \multirow[t]{5}{*}{ Thymonin (72) } & & & & & M. spicata & {$[162,180]$} & T. striatus & {$[188]$} \\
\hline & & & & & M. x piperita & {$[162,180]$} & & \\
\hline & & & & & M. suaveolens & [180] & & \\
\hline & & & & & M. pulegium & [180] & & \\
\hline & & & & & M. longifolia & [180] & & \\
\hline \multirow[t]{3}{*}{ Pebrellin (73) } & & & & & M. citrata & [162] & T. striatus & [188] \\
\hline & & & & & M. aquatica & {$[162]$} & & \\
\hline & & & & & M. x piperita & $\begin{array}{l}{[104,162,} \\
180]\end{array}$ & & \\
\hline \multirow[t]{3}{*}{ Gardenin B (74) } & & & & & M. citrata & [162] & T. striatus & {$[188]$} \\
\hline & & & & & M. aquatica & {$[162]$} & & \\
\hline & & & & & M. x piperita & $\begin{array}{l}{[104,162,} \\
180]\end{array}$ & & \\
\hline
\end{tabular}




\section{2 | INTRODUCTION}

\begin{tabular}{|c|c|c|c|c|}
\hline \multirow[t]{2}{*}{ Desmethylnobiletin (75) } & M. spicata & [162] & T. striatus & {$[188]$} \\
\hline & M. x piperita & {$[162,180]$} & & \\
\hline \multirow[t]{2}{*}{ Cirsilineol (76) } & M. spicata & {$[162]$} & T. vulgaris & [109] \\
\hline & & & T. herba-barona & [131] \\
\hline \multirow[t]{2}{*}{ Sorbifolin (77) } & M. x piperita & {$[180]$} & T. herba-barona & [131] \\
\hline & M. pulegium & {$[180]$} & & \\
\hline \multirow[t]{3}{*}{ Salvigenin (78) } & M. citrata & {$[162,180]$} & T. striatus & {$[188]$} \\
\hline & M. aquatica & [162] & & \\
\hline & M. x piperita & {$[162,180]$} & & \\
\hline \multirow[t]{2}{*}{ Ladanein (79) } & M. x piperita & {$[162,180]$} & T. striatus & [188] \\
\hline & M. pulegium & {$[180]$} & & \\
\hline \multirow[t]{3}{*}{ Cirsimaritin (80) } & & & T. serpyllum & [114] \\
\hline & & & T. herba-barona & [131] \\
\hline & & & T. vulgaris & [106] \\
\hline \multirow[t]{2}{*}{ Xanthomicrol (81) } & M. x piperita & {$[180]$} & T. striatus & {$[188]$} \\
\hline & & & T. herba-barona & [131] \\
\hline Sideritoflavone (82) & M. spicata & [162] & T. herba-barona & [131] \\
\hline
\end{tabular}


<smiles>[R6]Oc1ccc(-c2cc(=O)c3c(O[R6])c([R])c(O[R2])c([R3])c3o2)cc1[R6]</smiles>

Apigenin derivatives

$55 \mathrm{R}_{2}=$ Glc $\mathrm{R}_{1}=\mathrm{R}_{3}=\mathrm{R}_{4}=\mathrm{R}_{5}=\mathrm{R}_{6}=\mathrm{H}$

$56 \mathrm{R}_{6}=$ Glc $\mathrm{R}_{1}=\mathrm{R}_{2}=\mathrm{R}_{3}=\mathrm{R}_{4}=\mathrm{R}_{5}=\mathrm{H}$

$57 \mathrm{R}_{5}=\mathrm{Glc} \mathrm{R}_{1}=\mathrm{R}_{2}=\mathrm{R}_{3}=\mathrm{R}_{4}=\mathrm{R}_{6}=\mathrm{H}$

$58 \mathrm{R}_{3}=$ GlcAra $\mathrm{R}_{1}=\mathrm{R}_{2}=\mathrm{R}_{4}=\mathrm{R}_{5}=\mathrm{R}_{6}=\mathrm{H}$

$59 \mathrm{R}_{2}=\mathrm{GlcU} \mathrm{R}_{1}=\mathrm{R}_{3}=\mathrm{R}_{4}=\mathrm{R}_{5}=\mathrm{R}_{6}=\mathrm{H}$

$60 \mathrm{R}_{2}=$ Rut $\mathrm{R}_{1}=\mathrm{R}_{3}=\mathrm{R}_{4}=\mathrm{R}_{5}=\mathrm{R}_{6}=\mathrm{H}$

$61 \mathrm{R}_{3}=\mathrm{Glc} \mathrm{R}_{1}=\mathrm{R}_{2}=\mathrm{R}_{4}=\mathrm{R}_{5}=\mathrm{R}_{6}=\mathrm{H}$

$62 \mathrm{R}_{1}=\mathrm{R}_{3}=\mathrm{Glc}_{2}=\mathrm{R}_{4}=\mathrm{R}_{5}=\mathrm{R}_{6}=\mathrm{H}$

$63 \quad \mathrm{R}_{1}=\mathrm{R}_{2}=\mathrm{R}_{3}=\mathrm{R}_{4}=\mathrm{R}_{5}=\mathrm{R}_{6}=\mathrm{H}$

\section{Luteolin derivatives}

$44 \mathrm{R}_{1}=\mathrm{R}_{3}=\mathrm{R}_{5}=\mathrm{R}_{6}=\mathrm{H} \mathrm{R}_{2}=$ Glc $\mathrm{R}_{4}=\mathrm{OH}$

$45 \mathrm{R}_{1}=\mathrm{R}_{3}=\mathrm{R}_{5}=\mathrm{R}_{6}=\mathrm{H} \mathrm{R}_{2}=$ GlcAc $\mathrm{R}_{4}=\mathrm{OH}$

$46 \mathrm{R}_{1}=\mathrm{R}_{3}=\mathrm{R}_{5}=\mathrm{R}_{6}=\mathrm{H} \mathrm{R}_{2}=$ Glc $\mathrm{R}_{4}=$ OGlc

$47 \quad \mathrm{R}_{1}=\mathrm{R}_{3}=\mathrm{R}_{5}=\mathrm{R}_{6}=\mathrm{H} \mathrm{R}_{2}=$ GlcFer $\mathrm{R}_{4}=\mathrm{OH}$

$48 \mathrm{R}_{1}=\mathrm{R}_{3}=\mathrm{R}_{5}=\mathrm{R}_{6}=\mathrm{H} \mathrm{R}_{2}=\mathrm{GlcU} \mathrm{R}_{4}=\mathrm{OH}$

$49 \mathrm{R}_{1}=\mathrm{R}_{3}=\mathrm{R}_{5}=\mathrm{R}_{6}=\mathrm{H} \mathrm{R}_{2}=\mathrm{CH}_{3} \mathrm{GlcU} \mathrm{R}_{4}=\mathrm{OH}$

$50 \mathrm{R}_{1}=\mathrm{R}_{3}=\mathrm{R}_{5}=\mathrm{R}_{6}=\mathrm{H} \mathrm{R}_{2}=$ GlcU R $\mathrm{R}_{4}=\mathrm{OGlcU}$

$51 \mathrm{R}_{1}=\mathrm{R}_{3}=\mathrm{R}_{5}=\mathrm{R}_{6}=\mathrm{H} \mathrm{R}_{2}=$ Rut $\mathrm{R}_{4}=\mathrm{OH}$

$52 \mathrm{R}_{3}=$ GlcAra $\mathrm{R}_{4}=\mathrm{OH} \mathrm{R} \mathrm{R}_{1}=\mathrm{R}_{2}=\mathrm{R}_{5}=\mathrm{R}_{6}=\mathrm{H}$

$53 \mathrm{R}_{4}=\mathrm{OH} \mathrm{R}=\mathrm{R}_{2}=\mathrm{R}_{3}=\mathrm{R}_{5}=\mathrm{R}_{6}=\mathrm{H}$

$54 \mathrm{R}_{4}=\mathrm{OH} \mathrm{R}_{2}=\mathrm{CH}_{3} \mathrm{R}_{1}=\mathrm{R}_{3}=\mathrm{R}_{5}=\mathrm{R}_{6}=\mathrm{H}$<smiles>[R]Oc1cc(O)c2c(=O)cc(-c3cc([R5])c([R16])c([R5])c3)oc2c1[R]</smiles>

Isoscutellarein derivatives

\section{Other flavones (OMe)}

$71 \mathrm{R}_{1}=\mathrm{OH} \mathrm{R} \mathrm{R}_{2}=\mathrm{CH}_{3} \mathrm{R}_{3}=\mathrm{OCH}_{3} \mathrm{R}_{4}=\mathrm{R}_{5}=\mathrm{R}_{6}=\mathrm{H}$

$72 \mathrm{R}_{1}=\mathrm{OH} \mathrm{R}_{2}=\mathrm{CH}_{3} \mathrm{R}_{3}=\mathrm{R}_{4}=\mathrm{OCH}_{3} \mathrm{R}_{5}=\mathrm{R}_{6}=\mathrm{H}$

$73 \mathrm{R}_{1}=\mathrm{OH} \mathrm{R}=\mathrm{R}_{5}=\mathrm{CH}_{3} \mathrm{R}_{3}=\mathrm{OCH}_{3} \mathrm{R}_{4}=\mathrm{R}_{6}=\mathrm{H}$

$74 \mathrm{R}_{1}=\mathrm{R}_{3}=\mathrm{OCH}_{3} \mathrm{R}_{2}=\mathrm{R}_{5}=\mathrm{CH}_{3} \mathrm{R}_{4}=\mathrm{R}_{6}=\mathrm{H}$

$75 \mathrm{R}_{1}=\mathrm{R}_{3}=\mathrm{R}_{4}=\mathrm{OCH}_{3} \mathrm{R}_{2}=\mathrm{R}_{5}=\mathrm{CH}_{3} \mathrm{R}_{6}=\mathrm{H}$

$76 \mathrm{R}_{1}=\mathrm{R}_{4}=\mathrm{OCH}_{3} \mathrm{R}_{2}=\mathrm{CH}_{3} \mathrm{R}_{3}=\mathrm{R}_{5}=\mathrm{R}_{6}=\mathrm{H}$

$77 \mathrm{R}_{1}=\mathrm{OHR}_{2}=\mathrm{CH}_{3} \mathrm{R}_{3}=\mathrm{R}_{4}=\mathrm{R}_{5}=\mathrm{R}_{6}=\mathrm{H}$

$78 \mathrm{R}_{1}=\mathrm{OCH}_{3} \mathrm{R}_{2}=\mathrm{R}_{5}=\mathrm{CH}_{3} \mathrm{R}_{3}=\mathrm{R}_{4}=\mathrm{R}_{6}=\mathrm{H}$

$79 \mathrm{R}_{1}=\mathrm{OHR}_{2}=\mathrm{R}_{5}=\mathrm{CH}_{3} \mathrm{R}_{3}=\mathrm{R}_{4}=\mathrm{R}_{6}=\mathrm{H}$

$80 \mathrm{R}_{1}=\mathrm{OCH}_{3} \mathrm{R}_{2}=\mathrm{CH}_{3} \mathrm{R}_{3}=\mathrm{R}_{4}=\mathrm{R}_{5}=\mathrm{R}_{6}=\mathrm{H}$

$81 \mathrm{R}_{1}=\mathrm{R}_{3}=\mathrm{OCH}_{3} \mathrm{R}_{2}=\mathrm{CH}_{3} \mathrm{R}_{4}=\mathrm{R}_{5}=\mathrm{R}_{6}=\mathrm{H}$

$82 \mathrm{R}_{1}=\mathrm{R}_{3}=\mathrm{OCH}_{3} \mathrm{R}_{2}=\mathrm{CH}_{3} \mathrm{R}_{4}=\mathrm{OH} \mathrm{R} \mathrm{R}_{5}=\mathrm{R}_{6}=\mathrm{H}$

$64 \mathrm{R}_{2}=\mathrm{OGlcU} \mathrm{R}_{1}=\mathrm{R}_{3}=\mathrm{R}_{5}=\mathrm{H} \mathrm{R}_{4}=\mathrm{OH}$

\section{Hypolaetin derivatives}

$65 \mathrm{R}_{1}=\mathrm{R}_{5}=\mathrm{H} \mathrm{R}_{2}=\mathrm{OGlcU} \mathrm{R}_{3}=\mathrm{R}_{4}=\mathrm{OH}$

$66 \mathrm{R}_{1}=\mathrm{R}_{5}=\mathrm{H} \mathrm{R}_{2}=\mathrm{OGlcU} \mathrm{R}_{3}=\mathrm{R}_{4}=\mathrm{OCH}_{3}$

Ara- Arabinosyl unit; Fer- Feruloyl unit;Glc- Glucosyl unit; GlcU- Glucuronyl unit; Rut- Rutinosyl unit

Figure 13 - Chemical structures of flavones reported in Cytisus, Lamium, Lavandula, Leonurus, Mentha and Thymus plants. 


\subsubsection{Flavonols}

Flavonols are a class of flavonoids characterized by a 3-hydroxyflavone backbone (Fig. 14).<smiles>O=c1c(O)c(-c2ccccc2)oc2ccccc12</smiles>

Figure 14 - General structure of flavonols.

Flavonols occur as major phenolic constituents in Leonurus [54, 55, 125, 126, 133 , 189] and Lamium species [17, 26, 139, 173] and are also present in Cytisus plants [4, 16]. From those, and as expected, quercetin and kaempferol derivatives are the most representative ones.

Quercetin derivatives are the most reported flavonols in Leonurus genus. As can be observed in Table 4, mono and di-glycosidic derivatives of this flavonol (83-95) have been described for a great number of Leonurus plants [54, 126, 127, 133, 159, 182, 190]. These are also detectable in Lamium album, L. maculatum and L. amplexicaule [17, 26, 139, 160, 171, 173]. Similar quercetin derivatives in Cytisus genus have been described for $C$. scoparius and C. multiflorus $[4,16]$. From all the glycosidic quercetin derivatives, rutin is the most common. This has been reported to account for 11.9, 0.35 and $4.1 \mathrm{mg} / \mathrm{g}$ of Leonurus sibiricus, Lamium maculatum and C. multiflorus dry plants, respectively [16, 139, 173, 182]. Besides these derivatives, the quercetin aglycone (95) has been described in all the six target plant genera, with the exception of Lavandula.

Kaempferol derivatives are also important constituents of Cytisus, Lamium and Leonurus plants. The aglycone form (96) together with several glycosydic derivatives (97-103), have been described in C. scoparius, C. multiflorus $[4,16]$ and in the Leonurus species L. cardiaca [54, 133] and L. japonicas [125, 126]. Quantification data indicated that kaempferol hexosides represented approximately $15 \%$ of the total phenolics in C. multiflorus $(5.7 \mathrm{mg} / \mathrm{g}$ of dry plant). Moreover, distinct O-kaempferol glycosidic derivatives have been reported in $L$. album $[26,171]$ and in $L$. amplexicaule [160]. Moreover, rhamnetin (104) has been described to occur in C. scoparius [4] and ishoramnetin $(105)$ and $O$-glycosidic derivatives $(106,107)$ have been reported in $T$. vulgaris and/or L japonicus species [106, 125]. 
Table 4 - Flavonols of Cytisus, Lamium, Lavandula, Leonurus, Mentha and Thymus genera.

\begin{tabular}{|c|c|c|c|c|c|c|c|c|c|c|}
\hline \multirow{2}{*}{$\begin{array}{l}\text { Compound } \\
\text { Flavonols }\end{array}$} & \multicolumn{2}{|c|}{ Cytisus species } & \multicolumn{2}{|c|}{ Lamium species } & \multirow[t]{2}{*}{ Lavandula species } & \multicolumn{2}{|c|}{ Leonurus species } & \multirow[t]{2}{*}{ Mentha species } & \multicolumn{2}{|c|}{ Thymus species } \\
\hline & & & & & & & & & & \\
\hline \multirow[t]{3}{*}{ Quercetin-3-O-glucoside (83) } & & & L. album & [26] & L. $x$ intermedia & L. cardiaca & [54] & & & \\
\hline & & & & & & L. sibiricus & {$[127,182]$} & & & \\
\hline & & & & & & L. japonicus & {$[125,126]$} & & & \\
\hline Quercetin-7-O-glucoside (84) & & & L. album & [17] & & L. cardiaca & [54] & & T. vulgaris & {$[106]$} \\
\hline \multirow[t]{2}{*}{ Quercetin-3-O-galactoside (85) } & & & & & & L. cardiaca & {$[54,133]$} & & & \\
\hline & & & & & & L. sibiricus & [182] & & & \\
\hline Quercetin-3-O-rhamnoside (86) & C. scoparius & {$[4]$} & L. maculatum & {$[17]$} & & L. cardiaca & [54] & & & \\
\hline Quercetin dihexoside (87) & C. multiflorus & [16] & & & & & & & & \\
\hline Quercetin acetyldihexoside (88) & C. multiflorus & [16] & & & & & & & & \\
\hline $\begin{array}{l}\text { 3'- } O \text {-methylquercetin-3-O- } \\
\text { rutinoside (89) }\end{array}$ & & & L. maculatum & {$[17,173]$} & & & & & & \\
\hline $\begin{array}{l}\text { Quercetin } \\
\text { 3-O- } \alpha \text {-L-rhamnopyranosyl- } \\
(1 \rightarrow 6)-\beta \text {-D-galactopyranoside } \\
(\mathbf{9 0})\end{array}$ & & & & & & L. sibiricus & {$[182]$} & & & \\
\hline $\begin{array}{l}\text { Quercetin-3-O-[3-(4-hydroxy- } \\
\text { 3,5-dimethoxybenzyl)- } \alpha \text {-L- } \\
\text { rhamnopyranosyl]-( }(\rightarrow 6) \text { - } \beta \text {-D- } \\
\text { galactopyranoside }(\mathbf{9 1 )}\end{array}$ & & & & & & L. heterophyllus & [190] & & & \\
\hline \multirow[t]{2}{*}{ Rutin (92) } & C. scoparius & [4] & L. album & {$[26,171]$} & & L. cardiaca & $\begin{array}{l}{[54,55,} \\
133]\end{array}$ & M. x piperita & T. vulgaris & {$[106]$} \\
\hline & C. multiflorus & [16] & L. maculatum & {$[139,173]$} & & L. sibiricus & {$[127,182]$} & & & \\
\hline 2"'’-Syringylrutin (93) & & & & & & L. japonicus & {$[126,159]$} & & & \\
\hline $\begin{array}{l}\text { Quercetin-3-O-[B-D- } \\
\text { glucopyranosyl- }(1 \rightarrow 4)][\alpha-\mathrm{L}- \\
\text { rhamnopyranosyl- }(1 \rightarrow 6)]-\beta-\mathrm{D}- \\
\text { glucopyranoside }(\mathbf{9 4})\end{array}$ & & & L. amplexicaule & {$[160]$} & & & & & & \\
\hline \multirow[t]{2}{*}{ Quercetin (95) } & C. scoparius & [4] & L. album & {$[26,171]$} & & L. cardiaca & [133] & M. x piperita & T. vulgaris & {$[106,121]$} \\
\hline & & & & & & L. sibiricus & [182] & & T. serpyllum & [121] \\
\hline Kaempferol (96) & C. scoparius & [4] & & & & L. cardiaca & [133] & & & \\
\hline Kaempferol- $O$-hexoside (97) & C. multiflorus & [16] & L. album & {$[171]$} & & L. cardiaca & {$[54]$} & & & \\
\hline
\end{tabular}




\section{6 | INTRODUCTION}

\begin{tabular}{|c|c|c|c|c|c|c|c|c|c|c|c|c|}
\hline Kaempferol acetylhexoside (98) & C. multiflorus & [16] & & & & & & & & & & \\
\hline $\begin{array}{l}\text { Kaempferol malonyl glucoside } \\
\text { (99) }\end{array}$ & & & & & L. angustifolia & {$[157]$} & & & & & & \\
\hline $\begin{array}{l}\text { Kaempferol-3-coumaryl } \\
\text { glucoside (100) }\end{array}$ & & & L. album & {$[26,171]$} & L. angustifolia & {$[157]$} & L. japonicus & {$[125,126]$} & & & & \\
\hline Kaempferol-7-O-rutinoside (101) & & & L. amplexicaule & [160] & & & L. japonicus & [126] & M. x piperita & [116] & & \\
\hline $\begin{array}{l}\text { 4'-methoxykaempferol-7-O- } \\
\text { rutinoside (102) }\end{array}$ & & & & & & & & & M. x piperita & {$[116]$} & & \\
\hline $\begin{array}{l}\text { Kaempferol-3- } O \text {-[B-D- } \\
\text { glucopyranosyl- }(1 \rightarrow 4)][\alpha-\mathrm{L}- \\
\text { rhamnopyranosyl- }(1 \rightarrow 6)]-\beta-\mathrm{D}- \\
\text { glucopyranoside }(\mathbf{1 0 3})\end{array}$ & & & L. amplexicaule & [160] & & & & & & & & \\
\hline Rhamnetin (104) & C. scoparius & [4] & & & & & & & & & & \\
\hline Isorhamnetin (105) & & & & & & & & & & & T. vulgaris & [106] \\
\hline Isorhamnetin- $O$-glucoside (106) & & & & & & & & & & & T. vulgaris & [106] \\
\hline $\begin{array}{l}\text { Isorhamnetin-3-O-rutinoside } \\
\text { (107) }\end{array}$ & & & & & & & L. japonicus & {$[125]$} & & & & \\
\hline
\end{tabular}


<smiles>[R]Oc1cc(O)c2c(=O)c(O[R3])c(-c3ccc(O[R8])c([R])c3)oc2c1</smiles>

Quercetin derivatives

$$
\begin{array}{ll}
\mathbf{8 3} & \mathrm{R}_{2}=O H R_{1}=R_{3}=H R_{4}=G l c \\
\mathbf{8 4} & R_{1}=G l c R_{2}=O H R_{3}=R_{4}=H \\
\mathbf{8 5} & R_{2}=O H R_{1}=R_{3}=H_{4}=G a l \\
\mathbf{8 6} & R_{2}=O H R_{1}=R_{3}=H R_{4}=\text { Rha } \\
\mathbf{8 7} & R_{1}=R_{4}=G l c R_{2}=O H R_{3}=H \\
\mathbf{8 8} & R_{1}=G_{c} R_{4}=G_{c} A c R_{2}=O H R_{3}=H \\
\mathbf{8 9} & R_{1}=H R_{2}=O C H_{3} R_{3}=H R_{4}=\text { Rut } \\
\mathbf{9 0} & R_{1}=H R_{2}=O H R_{3}=H R_{4}=\text { RhaGal } \\
\mathbf{9 1} & R_{1}=H_{2}=O H R_{3}=H R_{4}=\text { SyrRhaGal } \\
\mathbf{9 2} & R_{1}=R_{3}=H R_{2}=O H R_{4}=\text { Rut } \\
\mathbf{9 3} & R_{1}=R_{3}=H R_{2}=O H R_{4}=\text { SyrRut } \\
\mathbf{9 4} & R_{1}=R_{3}=H R_{2}=O H R_{4}=\text { GlcRut } \\
\mathbf{9 5} & R_{1}=R_{3}=R_{4}=H R_{2}=O H
\end{array}
$$

\section{Kaempferol derivatives}

$96 \mathrm{R}_{1}=\mathrm{R}_{2}=\mathrm{R}_{3}=\mathrm{R}_{4}=\mathrm{H}$

$97 \quad R_{1}=R_{2}=R_{3}=H R_{4}=\mathrm{Glc}$

$98 \mathrm{R}_{1}=\mathrm{R}_{2}=\mathrm{R}_{3}=\mathrm{H} \mathrm{R}_{4}=\mathrm{GlcAc}$

$99 \mathrm{R}_{1}=\mathrm{R}_{2}=\mathrm{R}_{3}=\mathrm{H} \mathrm{R}_{4}=\mathrm{GlcMal}$

$100 \mathrm{R}_{1}=\mathrm{R}_{2}=\mathrm{R}_{3}=\mathrm{H} \mathrm{R}_{4}=$ GlcCou

$101 R_{1}=$ Rut $R_{2}=R_{3}=R_{4}=H$

$102 R_{1}=$ Rut $R_{2}=R_{4}=H_{3} R_{3}=C_{3}$

$103 R_{1}=R_{2}=R_{3}=H_{4}=$ GlcRut

\section{Rhamnetin derivatives}

$104 \mathrm{R}_{1}=\mathrm{CH}_{3} \mathrm{R}_{3}=\mathrm{R}_{4}=\mathrm{H} \mathrm{R}_{2}=\mathrm{OH}$

$105 \mathrm{R}_{1}=\mathrm{HR}_{2}=\mathrm{OCH}_{3} \mathrm{R}_{3}=\mathrm{R}_{4}=\mathrm{H}$

$106 \mathrm{R}_{1}=\mathrm{HR}_{2}=\mathrm{OCH}_{3} \mathrm{R}_{3}=\mathrm{HR}_{4}=\mathrm{Glc}$

$107 \mathrm{R}_{1}=\mathrm{HR}_{2}=\mathrm{OCH}_{3} \mathrm{R}_{3}=\mathrm{H} \mathrm{R}_{4}=$ Rut

Ac- Acetyl unit; Cou- Coumaroyl unit; Gal- Galactosyl unit; Glc- Glucosyl unit; Mal- Mallonyl unit; Rut- Rutinosyl unit; RhaRhamnosyl unit; Syr- Syringyl unit

Figure 15 - Chemical structures of flavonols reported in Cytisus, Lamium, Lavandula, Leonurus, Mentha and Thymus plants. 


\subsubsection{Flavanones}

Flavanones are characterized by the absence of the double bond between the 2- and 3-positions and the presence of a chiral center at the 2-position of the C-ring (Fig. 16).

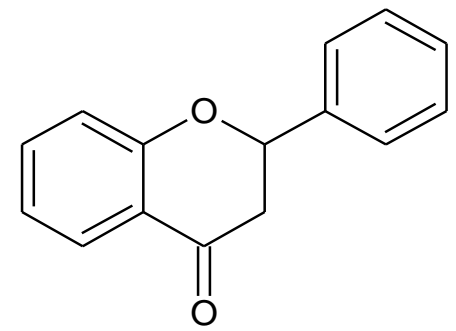

Figure 16 - General structure of flavanones.

In the six studied genera, flavanones (mainly eriodictyol and naringenin derivatives) are present in Mentha, Thymus and Lavandula plants, while, to the best of to the author's knowledge, this class of compounds have never been reported in Lamium and Cytisus species.

Mentha plants are enriched in O-glycosydic derivatives of eriodictyol, being the eriocitrin (eriodictyol-7-O-rutinoside) (108) the most widespread and the most abundant. Concentrations of about $16 \mathrm{mg} / \mathrm{g}$ of dry plant have been found in $M . x$ piperita $[61,62,93,104,107,116,120,152]$ and amounts between 2.5 and $7.4 \mathrm{mg} / \mathrm{g}$ dry plant have been described for $M . x$ dalmatica, M. spicata and M. "Native Wilmet" [61]. Eriocitrin was also found in T. serpyllum and in T. vulgaris (1.2 $\mathrm{mg} / \mathrm{g}$ of dry plant). These two species also contain the O-glucuronide derivative of eriodictyol (109) [100, $114,135]$. On the other hand, eridioctyol-O-glucoside (110) is the unique eriodictyol derivative described in Lavandula plants, namely in L. $x$ intermedia. This compound, together with pinocembrin (111) (detected in L. viridis species), are the only flavanones described in Lavandula species [149, 168].

Concerning the eriodictyol aglycone (112), this has been described to occur in Thymus and Mentha genus. In more detail, this form has been detected in T. serpyllum, $T$. webbianus and T. herba-barona [114, 131, 153, 154], T. vulgaris (1.5 mg/g of dry plant) $[108,119,154]$ and in $M . x$ piperita $(0.1$ to $0.5 \mathrm{mg} / \mathrm{g}$ of dry plant) $[61,120]$.

Despites less frequent than eriodictyol derivatives, naringenin derived compounds also occur in Thymus and in Mentha plants. The aglycone form (113) has been detected in $T$. vulgaris ( $0.4 \mathrm{mg} / \mathrm{g}$ of dry plant), in $T$. webbianus and in $T$. herba-barona [131, 153, 
154] and also shown to be present in low amounts in $M$. $x$ piperita and M. aquatica species [111, 191]. Instead, the 7-O-rutinoside derivative of naringenin (114) has been largely reported in $M . x$ piperita species $[103,107,116,120]$ and estimated to account for $0.3 \mathrm{mg} / \mathrm{g}$ of dry T. vulgaris [154]. A second naringenin-O-derivative, the naringenin7-O-glucoside (115), has also been reported in the latter species $(0.6 \mathrm{mg} / \mathrm{g}$ of dry plant) [154], in $M . x$ piperita, in $M$. arvensis (1.0 and $0.1 \mathrm{mg} / \mathrm{g}$ of dry plant in respectively) and in other Mentha species (Table 5) [61, 120, 152, 166].

Besides the most prevalent flavanones, hesperidin (hesperitin-7-O-rutinoside) (116) has been widely described in M. longifolia, M. x piperita (1.7 mg/g dry plant) [93, 100, 103, 104, 116, 181] and reported to amount for $1 \mathrm{mg} / \mathrm{g}$ of dry $T$. vulgaris plant [154]. Concerning the Leonurus species, the only flavanone described in this genus is hesperetin (117), which has been reported to occur in L. cardiaca extracts [133]. 
Table 5 - Flavanones of Lavandula, Leonurus, Mentha and Thymus genera.

\begin{tabular}{|c|c|c|c|c|c|c|}
\hline \multirow{2}{*}{$\begin{array}{l}\text { Compound } \\
\text { Flavanones }\end{array}$} & \multirow[t]{2}{*}{ Lavandula species } & \multirow[t]{2}{*}{ Leonurus species } & \multicolumn{2}{|c|}{ Mentha species } & \multicolumn{2}{|c|}{ Thymus species } \\
\hline & & & & & & \\
\hline \multirow[t]{9}{*}{ Eriocitrin (108) } & & & M. x piperita & $\begin{array}{l}{[61,62,93,} \\
104,107, \\
116,120, \\
152]\end{array}$ & T. vulgaris & $\begin{array}{l}{[121,154,} \\
192]\end{array}$ \\
\hline & & & M. aquatica & {$[166]$} & T. serpyllum & {$[121,154]$} \\
\hline & & & M. spicata & {$[61]$} & & \\
\hline & & & M. $x$ dalmatica & [61] & & \\
\hline & & & M. haplocalyx & [61] & & \\
\hline & & & M. "Morocco" & {$[166]$} & & \\
\hline & & & M. "Native Wilmet" & {$[61,166]$} & & \\
\hline & & & M. $x$ verticillata & {$[166]$} & & \\
\hline & & & $\begin{array}{l}\text { M. arvensis var. } \\
\text { japanensis }\end{array}$ & {$[61,166]$} & & \\
\hline \multirow[t]{2}{*}{ Eridioctyol-O-glucuronide (109) } & & & & & T. vulgaris & {$[100,135]$} \\
\hline & & & & & T. serpyllum & [114] \\
\hline Eridioctyol-O-glucoside (110) & $\begin{array}{ll}\text { L. } x \text { intermedia } & {[149]}\end{array}$ & & M. x piperita & $\begin{array}{l}{[103,104,} \\
120]\end{array}$ & T. vulgaris & {$[135,154]$} \\
\hline Pinocembrin (111) & $\begin{array}{ll}\text { L. viridis } \quad[168] \\
\end{array}$ & & & & & \\
\hline \multirow[t]{3}{*}{ Eriodictyol (112) } & & & $\begin{array}{l}\text { M. x piperita } \\
\text { M. "Native Wilmet" }\end{array}$ & $\begin{array}{l}{[61,120,} \\
152] \\
{[166]}\end{array}$ & $\begin{array}{l}\text { T. vulgaris } \\
\text { T. herba-barona }\end{array}$ & $\begin{array}{l}{[108,119,} \\
154] \\
{[131]}\end{array}$ \\
\hline & & & & & T. serpyllum & {$[114,154]$} \\
\hline & & & & & T. webbianus & [153] \\
\hline \multirow[t]{3}{*}{ Naringenin (113) } & & & M. x piperita & {$[111,120]$} & T. vulgaris & {$[154]$} \\
\hline & & & M. aquatica & [191] & T. herba-barona & [131] \\
\hline & & & & & T. webbianus & [153] \\
\hline Naringenin-7-O-rutinoside (114) & & & M. x piperita & $\begin{array}{l}{[103,107,} \\
116,120]\end{array}$ & T. vulgaris & {$[154]$} \\
\hline \multirow[t]{4}{*}{ Naringenin-7-O-glucoside (115) } & & & M. x piperita & {$[120,152]$} & T. vulgaris & {$[154]$} \\
\hline & & & M. haplocalyx & [61] & & \\
\hline & & & M. $x$ verticillata & [166] & & \\
\hline & & & M. arvensis & {$[61,166]$} & & \\
\hline Hesperidin (116) & & & $\begin{array}{l}\text { M. x piperita } \\
\text { M. longifolia }\end{array}$ & $\begin{array}{l}{[93,103,} \\
104,107, \\
116,120] \\
{[181]}\end{array}$ & T. vulgaris & [154] \\
\hline
\end{tabular}

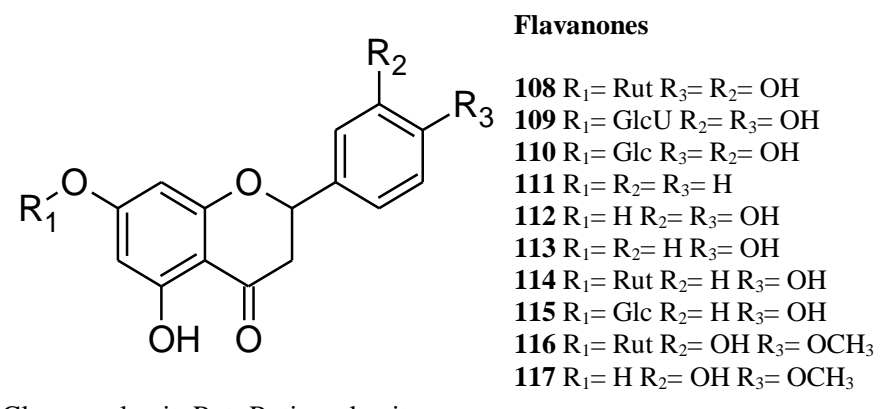

Glc- Glucosyl unit; GlcU- Glucuronyl unit; Rut- Rutinosyl unit

Figure 17 - Chemical structures of flavanones reported in Lavandula, Leonurus, Mentha and Thymus plants. 


\subsubsection{Isoflavones}

As observed in Fig. 18, this class of flavonoids is characterized by the presence of a double bond between the 2 and 3 positions and the attachment of B-ring to C-3, instead of C-2.<smiles>O=c1c(-c2ccccc2)coc2ccccc12</smiles>

Figure $18-$ General structure of isoflavones.

Isoflavone are the less representative class of flavonoids in the six genera (118-122) and to the author's knowledge, up to this moment, isoflavones have only been detected in Cytisus species (Table 6) [4, 113, 138].

Table 6 - Isoflavones of Cytisus genera.

\begin{tabular}{clc}
\hline Compound & \multicolumn{2}{c}{ Cytisus species } \\
\hline Isoflavones & & \\
\hline Daidzin (118) & C. albus & {$[113]$} \\
\hline Ononin (119) & C. nigricans & {$[138]$} \\
& C. albus & {$[113]$} \\
\hline Genistein (120) & C. albus & {$[113]$} \\
\hline Genistin (121) & C. albus & {$[113]$} \\
\hline Sarothamnoside (122) & C. scoparius & {$[4]$} \\
\hline
\end{tabular}<smiles>[R6]Oc1ccc(-c2coc3cc(O[R6])cc([R])c3c2=O)cc1</smiles>

$118 \mathrm{R}_{2}=$ Glc $\mathrm{R}_{1}=\mathrm{R}_{3}=\mathrm{H}$

$119 \mathrm{R}_{2}=\mathrm{Glc} \mathrm{R}_{1}=\mathrm{H} \mathrm{R}_{3}=\mathrm{CH}_{3}$

$120 \mathrm{R}_{1}=\mathrm{OH} \mathrm{R}=\mathrm{R}_{3}=\mathrm{H}$

$121 \mathrm{R}_{1}=\mathrm{OH} \mathrm{R} \mathrm{R}_{2}=\mathrm{Glc} \mathrm{R}_{3}=\mathrm{H}$

$122 \mathrm{R}_{1}=\mathrm{OH} \mathrm{R} \mathrm{R}_{2}=\mathrm{R}_{3}=$ ApiGlc

Api- Apiosyl unit; Glc- Glucosyl unit

Figure 19 - Chemical structures of isoflavones reported in Cytisus plants. 


\subsection{BenEFICIAL EFFECTS}

Several plants belonging to Cytisus, Lamium, Lavandula, Leonurus, Mentha and Thymus genera are used for decades in traditional medicine due to their claimed beneficial effects, including antioxidant, anti-inflammatory, antimicrobial, analgesic, neuroprotective and anti-carcinogenic. Following, a summary of the main beneficial properties associated to the plant genera herein in focus is presented and summarized in Table 7. Particular focus will be given to the antioxidant and anti-inflammatory properties, since these main explored plant properties in the investigated plant genus, and were also investigated in the practical part of the present work.

\subsubsection{Antioxidant activity}

Oxidative stress is a condition characterized by an imbalance between pro-oxidants and antioxidant defenses. The reactive oxygen and nitrogen species (ROS e.g. $\mathrm{O}_{2}{ }^{\bullet-}$, $\mathrm{OH}^{\bullet}, \mathrm{ROO}^{\circ}$ and $\mathrm{RNS}$, e.g. $\mathrm{NO}^{*}, \mathrm{ONOO}^{-}$) are generated in a variety of intracellular processes and their overproduction produces cell damage in lipids, proteins and DNA [193, 194]. The overproduction of these pro-oxidant agents is closely associated to aging processes and to the physiopathology of several diseases [195-197]. On the other hand, several compounds, namely antioxidants (e.g. polyphenols) can act counteracting oxidative stress through several mechanisms, e.g. free radical scavenging, electron or hydrogen atom donation or metal cation chelation [198]. In accordance to that, many of the beneficial activities of the plants (e.g. antiinflammatory) have been related with the antioxidant capacities of their phenolic compounds [199].

Several chemical in vitro methods have been developed in order to determine the total antioxidant capacity of plant extracts or of their individual phenolic components and includes, among others, the free radical 2,2-diphenyl-1-picrylhydrazyl (DPPH $\left.{ }^{\bullet}\right)$ scavenging, hydroxyl radical scavenging activity, the superoxide scavenging activity, the $\mathrm{NO}^{\bullet}$-scavenging activity, the reducing/antioxidant power (FRAP), the oxygen radical absorbance capacity (ORAC), the Trolox equivalence antioxidant capacity (TEAC), the B-carotene bleaching test and the inhibition of lipid peroxidation (Thiobarbituric Acid test). These assays are widely used, since they can give some clues on the extracts/phenolics antioxidant characteristics, besides being fast and simple [200, 201]. 
Besides the importance of these tests, cellular in vitro and in vivo animal models are particularly useful for further understanding the actions of these compounds in the human body. Due to the ethics issues, high costs and the time-consuming of the in vivo studies (including those with human), the evaluation of the antioxidant activity by means of cellular-based assays is a good alternative. A very common method of evaluating cellular antioxidant activity of compounds or of extracts, is through the use of the redox sensor dihydrodichlorofluorescein diacetate $\left(\mathrm{DCFH}_{2}\right)$, which is oxidized to fluorescent dichlorofluorescein (DCF) in the presence of $\mathrm{ROO}^{\circ}$. Another option is the assessment of antioxidant enzymes expression or activities (e.g. superoxide dismutase, glutathione peroxidase, glutathione reductase, catalases) vs inhibition of pro-oxidant enzymes (5-lipoxygenase, xanthine oxidase, nitric oxide synthase). The upregulation expression of antioxidant enzymes is a cellular strategy to reduce the oxidative status [202]. In accordance to that, other alternative of cellular-based assays is the assessment of activation (e.g. Nrf-2) vs repression of redox transcription factors such as Nf-kB.

The in vivo assays using animal models and human studies aim to evaluate the levels of oxidative stress biomarkers and usually engage the measurement of antioxidant compounds or enzyme levels, oxidation products or the ratio of oxidized to reduced form (e.g. GSSG/GSH). Despite the increase of in vivo studies in the last decades, these are still scarce for establishing the exact role of antioxidants in promoting the human health [203].

As shown in Table 7, several Lavandula species have been investigated for its antioxidant capacities through chemical methods. EC $_{50}$ values between 11.5 to 19.3 $\mu \mathrm{g} / \mathrm{mL}$ were reported for $\mathrm{DPPH}{ }^{\bullet}$ radical scavenging ability of $L . x$ intermedia 'Budrovka' and $L$. angustifolia hydroethanolic extracts, water extracts of $L$ latifolia and methanol extracts of L. coronopifolia and L. multifida [169, 204, 205], thus demonstrating high radical scavenging capacity for these plants. Higher EC $_{50}$ values $(40.6$ to $110.4 \mu \mathrm{g} / \mathrm{mL}$ ) have been determined for ethanolic extracts of $L$. $x$ intermedia Emeric ex Loiseleur, water extracts of $L$. dentata and several hydroethanolic extracts of $L$. hybrida and of subspecies of L. angustifolia [149, 206, 207]. Additionally, Lee et al. [115] reported strong antioxidant capacities both in $\mathrm{DPPH}^{\bullet}$ and $\mathrm{NO}^{\bullet}$-inhibition assays, which have been both correlated to their high phenolic content, for acetone extracts of $L$. allardii 'Rly' and L. stoechas.

For hydroethanolic L. hybrida and water L. stoechas extracts, the $\mathrm{EC}_{50}$ values in ferrous ion chelating activity assay are 49.9 and less than $20.0 \mu \mathrm{g} / \mathrm{mL}$, respectively [122, 207] while the radical scavenging ability of $\mathrm{ABTS}^{\bullet+}$ in lavender extracts varies between 2.5 
$\mu \mathrm{M}$ of Trolox equivalents, for $L$. vera [208] and about $1 \mathrm{mM}$ of Trolox equivalents for ethanolic and water L. officinalis extracts $[123,209]$.

The antioxidant activity of Lamium album enriched phenolic extracts has also been extensively assessed by the $\mathrm{DPPH}^{\bullet}$ assay, and also through the evaluation of their scavenging effects on superoxide and hydroxyl radicals [210, 211]. In general, the authors have closely associated the phenolic content and/or composition of the extracts to their antioxidant properties [18, 212], as well as to their health benefits [26, 27]. In more detail, $\mathrm{EC}_{50}$ values between 64.5 and $96.2 \mu \mathrm{g} / \mathrm{mL}$ have been estimated for the $\mathrm{DPPH}^{\bullet}$ radical scavenging ability by the methanolic extracts of $L$. eriocephalum subsp. eriocephalum, L. garganicum subsp. laevigatum and L. purpureum var. purpureum [213], and $\mathrm{EC}_{50}$ values of 14.1 to $63.9 \mu \mathrm{g} / \mathrm{mL}$ have been obtained for five of the isolated phenolics from L. amplexicaule [160]. The phosphomolybdenum reduction assay [214] showed an antioxidant potential of 131.2 and about $250 \mathrm{AAE} \mathrm{mg} / \mathrm{g}$ for aqueous methanolic and methanolic $L$. maculatum and $L$. album extracts, respectively $[18,50]$.

Phenolic compounds in Mentha have also been associated to their beneficial properties, supporting their ethnopharmacological usage. The antioxidant ability of Mentha extracts has been shown by assessing free radical scavenging activity against $\mathrm{DPPH}^{\bullet}$, as well as by evaluating the lipid peroxidation protective activity, using both the thiobarbituric acid, B-carotene bleaching methods and iron(III) reduction and iron(II) chelation [73, 152, 204, 215]. The cultivar M. x piperita "Frantsila" has been described as a good source of antioxidants compounds [61] as well as the species $M$. spicata, $M$. aquatica and $M$. suaveolens. The $\mathrm{DPPH}^{\bullet}$ scavenging $\mathrm{EC}_{50}$ values for aqueous ethanolic or methanolic extracts of these two latter species have been estimated to be about $30 \mu \mathrm{g} / \mathrm{mL}[73,204,215]$. Besides these spectrometric assays, the protective effects against oxidative stress of methanolic $M$. $\times$ piperita and $M$. aquatica extracts were proved in hydrogen-peroxide-induced toxicity in PC12 cells (Rat pheochromocytoma cells) [216]. An in vivo study performed with ethanolic extract of $M$. pulegium by Jain and colleagues [217] showed that at a dose of $600 \mathrm{mg} / \mathrm{kg}$, the extract significantly improved the glutathione, SOD, catalase, and peroxidase levels, when compared to the control group.

Water, methanolic, ethanolic or water extracts of $T$. vulgaris, i.e., the most investigated Thymus species, have been shown to have $\mathrm{DPPH}^{\bullet}$ radical $\mathrm{EC}_{50}$ values between 33.3 and $56.1 \mu \mathrm{g} / \mathrm{mL}$. Other chemical in vitro tests, including the reducing power, hydrogen peroxide scavenging activity, hydroxyl radical scavenging activity also have been shown positive results for the antioxidant capacity of this Thymus species [15, 204, 
218, 219]. Moreover, different studies in methanolic extracts of $T$. leucotrichius, water extracts of $T$. camphoratus, $T$. carnosus, $T$. mastichina and $T$. $x$ citriodorus have been proved the high antioxidant properties of the species [15, 220, 221].

Considering the Leonurus genus, several studies have highlighted the antioxidant properties of aqueous methanolic or methanolic extracts of $L$. cardiaca, in particular by demonstrating their efficient ability to chelate iron $[50,51,53,56,186]$. The DPPH scavenging test performed with methanolic extracts of this species estimated $E_{50}$ values varying in between 27 and $144 \mu \mathrm{g} / \mathrm{mL}$. Other antioxidant species includes $L$. japonicas, in which the antioxidant property of its methanolic extract has been evaluated trough the ferric thiocyanate method [125]. Furthermore, an in vivo study performed by Lee and colleagues [222] also allowed concluding that the hydroethanolic extract of $L$. sibiricus supplementation attenuates the intracellular oxidative stress induced in rats with an atherogenic diet. Overall, the protective effect has been speculated to be mediate through the enhancement of antioxidant enzymes and by the free radical scavenging activities of the plant.

From all the Cytisus species, C. scoparius is by far the most considered as an antioxidant agent, being this property frequently related to the high phenolic concentration of the plant [223]. $\mathrm{DPPH}^{\bullet} \mathrm{EC}_{50}$ values of 3.0 and $65.4 \mu \mathrm{g} / \mathrm{mL}$ have been obtained for hydroethanolic and ethanolic enriched phenolic extracts of $C$. scoparius, respectively while $\mathrm{EC}_{50}$ value of $70 \mu \mathrm{g} / \mathrm{mL}$ was obtained in B-carotene bleaching assay for an aqueous extract of the same species. Moreover, these exhibited good nitric oxide radical scavenging, superoxide anion radical scavenging, hydroxyl radical scavenging, antilipid peroxidation and high reducing power [98, 223, 224]. In good agreement with these results, an in vivo experiment showed the capacity of an hydroalcoholic extract of $C$. scoparius to increase the total antioxidant capacity, measuring by FRAP levels, to reduce thiobarbituric acid reactive substances (TBARS) and to increase SOD and catalase levels in liver and kidney [224], while other authors have concluded that the plant protects liver from oxidative stress induced by carbon tetrachloride in rats. In this particular case, the pretreatment with the plant extract lowered the serum glutamate oxaloacetate transaminases (SGOT), the serum glutamate oxaloacetate transaminases (SGPT), lactate dehydrogenase (LDH) and TBARS levels. Moreover, a significantly increase of reduced glutathione (GSH) and hepatic enzymes, like superoxide dismutase (SOD), catalase (CAT), glutathione peroxidase (GPx), glutathione reductase (GRD) and glutathione-s-transferase (GST) [4] was registered. 


\subsubsection{Anti-inflammatory activity}

Inflammation is a biological response of living tissues against injury or infection. Hence, it can be initiated by physical damage of tissue, toxic and chemical substances, as well as by microorganisms. The inflammatory process is characterized by a complex immunological process leading to a cascade activation, which includes the secretion of mediators as prostaglandin $\mathrm{E}_{2}\left(\mathrm{PGE}_{2}\right)$, the overproduction of reactive oxygen species (ROS) and reactive nitrogen species (RNS) such as nitric oxide (NO*), as well as the release of pro-inflammatory cytokines as interleukins (IL-1 $\beta, I L-6, I L-12)$, interferon (INF-y) and tumor necrosis factor (TNF- $\alpha$ ) [225, 226]. The release of these proinflammatory mediators is in turn stimulated by the inducible enzymes cyclooxygenase2 (COX-2), nitric oxide synthase (iNOS) and by lipoxygenase (LOX). On the other hand, this phenomenon is counteracted by anti-inflammatory cytokines (IL-4, IL-10, IL13) and TGF- $\beta$ [225-227]. Despite the protective role of inflammation, the reiterated inflammatory mechanisms are related to several medical disorders. Chronic inflammation is associated to various dysfunctions and pathologies such atherosclerosis, rheumatoid arthritis, asthma, obesity, diabetes, neurodegenerative diseases and even cancer [228].

At present, drugs to treat inflammatory disorders are classically in corticosteroid and nonsteroidal, both inhibiting the enzyme COX-1 and/or COX-2. Indeed, even despite new biological drugs with other cellular targets are now available in the market (e.g. infliximab, etanercept) for the treatment of rheumatology, dermatology and gastroenterology inflammatory ailments, their therapeutic usage entail high costs [229]. This fact, together with the high incidence of side effects on classical anti-inflammatory agents, stimulate the search for new safe anti-inflammatory drugs [225, 230]. In this way, plant extracts or their bioactive compounds (e.g. polyphenols) are also frequently assayed for anti-inflammatory properties [227].

As a first approach, chemical in vitro tests such $\mathrm{NO}^{\bullet}$ and $\mathrm{HOCl}$ scavenging are useful and routinely used for assessment of relevant anti-inflammatory activities. Additional information can be obtained from in vitro cell cultures tests after lipopolysaccharide stimulation of human monocytic leukemia cell line (THP-1 cells) or, more frequently, on monocyte/macrophage cell line RAW 264.7. Griess reaction is vastly used for measuring the nitrite accumulation in the culture supernatant on monocyte/macrophage cell line RAW 264.7. Moreover, some cellular proteins with important role in inflammation processes (e. g. iNOS, COX-2 and LOX) can be measured by Western Blot assay. Prostaglandin $\mathrm{E}_{2}\left(\mathrm{PGE}_{2}\right)$ and pro-inflammatory cytokines as TNF- $\alpha$ (tumor necrosis factor $\alpha$ ) and interleukine (e.g. IL-1 $\beta$, IL-2, IL-6) levels have also been 
estimated by enzyme immunoassay in macrophage culture medium [227]. Moreover, in vivo models include the paw edema induced by carrageenan, histamine, dextran and ear edema induced by toxics as croton oil, arachidonic-acid and xylene [231-234].

From the six plant genera focused in the present study, Lamium is the most exploited regarding their anti-inflammatory capacities. An hydroalcoholic extract of $L$. album has been demonstrated to inhibit the lipoxygenase activity [211] and to stimulate human skin fibroblasts, which are fundamental in tissue repair [26, 27]. Besides these, distinct extracts from L. garganicum subsp. laevigatum, L. garganicum subsp. pulchrum and $L$. purpureum var. purpureum have demonstrated good anti-inflammatory activities in distinct in vivo models [19], mostly in carrageenan-induced paw edema model. The inhibition of the croton oil-induced ear edema in mice model was used to evaluate topical anti-inflammatory activity of a $M$. aquatica hydroethanolic extract [73].

Anti-inflammatory properties have also been described for methanolic extracts of Cytisus aeolicus and Thymus richardii, through the inhibition of leukotriene B4 production in rat polymorphonuclear leukocytes [235]. Furthermore, Lee and colleagues [222] showed that an hydroethanolic extract of Leonurus sibiricus can suppress the activation of inflammatory mediators and this activity was confirmed in the carrageenan induced rat paw edema in vivo model [236]. Positive results were also obtained in the same model for the oil fraction of Lavandula angustifolia [237].

\subsubsection{Other beneficial activities}

Cytisus, Mentha, Thymus, Lavandula and Lamium plants have also been described to exhibit antimicrobial, anticancer, analgesic and neuroprotective activities. Some examples can be pointed, as follows.

From methanolic extracts of Lamium species, L. eriocephalum subsp. eriocephalum and $L$. tenuiflorum have been suggested as good antimicrobial agent against bacteria and fungi [213, 238] while Lavandula stoechas [239] and T. pallidus [239] methanolic extracts have antibacterial effects against Streptococcus pneumonia. Furthermore, the acetone extracts of the species $C$. aeolicus and $C$. capitatus exhibit synergic antibacterial effect when combined with typical antibiotics [240].

Regarding the anticancer activity, it is important to remark that $L$. album has been shown to exhibit potential anticancer effects. The cytotoxic effects of methanol and chloroform $L$. album extracts are partially caused by the retention of the cell cycle in $\mathrm{G}_{2}$ period, as demonstrated on the lung cancer cell line A549 [241]. Additionally, M. 
spicata, M. × piperita ethanolic or methanolic extracts, respectively, have shown antitumorogenic properties in human prostate (PC-3) and colon (SW-480) cancer cell lines [242, 243] while methanolic $T$. vulgaris extracts have been shown the same property in the last cell line [242], and their metabolites have been shown antimutagenic activity [244]. Other study indicated that ethyl acetate extract of $T$. quinquecostatus as an antitumor agent in human leukemia cell line [245].

As suggested by Akkol et al. [19], Lamium garganicum subsp. laevigatum, L. garganicum subsp. pulchrum and L. purpureum var. purpureum are good candidates for in vivo antinociceptive agents, which has been attributed to the presence of iridoids and phenolic compounds in the plant extracts. The effect was measured by the antinociceptive activity $p$-benzoquinone (PBQ)-induced writhing test in mice. Besides these plants, ethanolic extracts of $T$. satureioides, $T$. maroccanus and $T$. leptobotrys have been described to exhibit analgesic activities, thus supporting their traditional use in the relief of some pains [246]. The same property has been demonstrated for Leonurus sibiricus methanolic extract in acetic acid-induced writhing in mice [236].

Some species of the six genera herein in focus have been shown to be neuroprotective. A methanolic extract of Lavandula viridis emerged as an inhibitor of acetylcholinesterase (AChE) and butyrylcholinesterase (BChE), as demonstrated by Ellman's method [247], in in vitro and in vivo models [168]. Water extracts of $L$. angustifolia $\mathrm{L}$. have showed efficient neuronal protection against glutamate toxicity [248] and positive influence in the cognitive performance, enhancing memory consolidation in a model of Alzheimer's disease [249]. Besides these species, several Mentha species have been demonstrated to have neuroprotective effects that can justify their traditional usage for counteracting central nervous system disorders. In more detail, methanolic extracts of $M . \times$ piperita and $M$. aquatica and an hydroethanolic extract of $M$. aquatica exerted monoamino oxidase A (MAO-A) inhibitory activity [72, 191, 216], an important condition of antidepressant agents. From those, M. aquatica has been shown to have the highest $\mathrm{GABA}_{A}$-receptor affinity, which is a crucial feature for sedative effects [216]. The described effects are attributed, almost in part, to its content in (S)-naringenin [72, 191].

Besides the above examples, it also should be mentioned that few examples of other beneficial activities (e.g. tyrosinase inhibitor, anticonvulsant, cardioprotective, anxiolytic and hepatoprotective) have been reported for the six plant target genera.

In this sense, $C$. scoparius has been shown in vivo sedative, moderate anxiolytic and liver protective effects, which have been frequently associated to its antioxidant activity 
$[4,6]$. Moreover, the water and aqueous acetone extracts of $L$. stoechas, L. latifolia, $L$. allardii and $L$. dentata species have been reported to exhibit a tyrosinase-inhibitory activity, rendering them potential application as whitening agents [115, 206]. Additionally, a hydromethanolic extract of the primer species has shown anticonvulsant activities in an epilepsy model and also antispasmodic and sedative effects, justifying their ethnopharmacological uses [250]. Furthermore, cardiovascular protective effects have been demonstrated by distinct assays for Leonurus sibiricus and T. pulegioides. In the first case, the reduction of plasma cholesterol, elevation of HDL cholesterol, and the decrement of the atherogenic index were obtained after supplementation with the hydroethanolic Leonurus extract for 14 weeks the mice, using a diet-induced hypercholesterolemia C57BL/6 mice model [222]. An ethanolic extract of $T$. pulegioides has shown the ability to increase the release of the vasorelaxant factors nitric oxide (NO) and the prostacyclin, thus showing cardiovascular protective effects [251]. 
Table 7 - Described effects in Cytisus, Lamium, Lavandula, Leonurus, Mentha and Thymus genera.

\begin{tabular}{|c|c|c|c|c|c|c|c|c|c|c|c|c|}
\hline Bioactivity & Cytisus species & & Lamium species & & Lavandula species & & Leonurus species & & Mentha species & & Thymus species & \\
\hline \multirow[t]{8}{*}{ Antioxidant } & C. scoparius & $\begin{array}{l}{[4,98,} \\
223,224]\end{array}$ & L. album & $\begin{array}{l}{[18,27,210,} \\
211]\end{array}$ & L. stoechas & [115] & L. japonicas & [125] & M. $\times$ piperita & [61] & T. vulgaris & $\begin{array}{l}{[15,204,} \\
218,219]\end{array}$ \\
\hline & & & L. eriocephalum & [213] & L. allardii & [115] & L. cardiaca & $\begin{array}{l}{[50,51,53,} \\
56,186]\end{array}$ & M. spicata & [215] & T. leucotrichius & [221] \\
\hline & & & L. garganicum & [213] & L. viridis & [168] & L. sibiricus & {$[222]$} & M. aquatica & [73] & T. $x$ citriodorus & [15] \\
\hline & & & L. purpureum & {$[18,213]$} & L. $x$ intermedia & [149] & & & M. suaveolens & [204] & T. pulegioides & [252] \\
\hline & & & L. amplexicaule & [160] & L.officinalis L. & $\begin{array}{l}{[123,209,} \\
253]\end{array}$ & & & & & T. mastichina & [220] \\
\hline & & & L. maculatum & [50] & L. latifolia & {$[110,204]$} & & & & & & \\
\hline & & & & & $\begin{array}{l}\text { L. stoechas } \\
\text { L. dentata }\end{array}$ & $\begin{array}{l}{[122]} \\
{[206]}\end{array}$ & & & & & & \\
\hline & & & & & $\begin{array}{l}\text { L. hybrida } \\
\text { L. vera }\end{array}$ & $\begin{array}{l}{[207]} \\
{[208]}\end{array}$ & & & & & & \\
\hline \multirow[t]{3}{*}{ Anti-inflammatory } & C. aeolicus & [235] & L. album & {$[26,27,211]$} & L. angustifolia & [237] & L. sibiricus & [236] & M. aquatica & [73] & T. richardii & [235] \\
\hline & & & L. garganicum & [19] & & & & & & & & \\
\hline & & & L. purpureum & [19] & & & & & & & & \\
\hline \multirow[t]{2}{*}{ Antimicrobial } & C. aeolicus & [240] & L. purpureum & [213] & L. stoechas & [239] & & & M. longifolia & [204] & T. vulgaris & [204] \\
\hline & C. capitatus & & L. tenuiflorum & {$[238,254]$} & & & & & M. $\times$ piperita & [204] & & \\
\hline \multirow[t]{2}{*}{ Anticancer } & & & L. album & [241] & & & & & M. $\times$ piperita & [242] & T. vulgaris & [242] \\
\hline & & & & & & & & & M. spicata & [243] & T. quinquecostatus & [245] \\
\hline \multirow[t]{3}{*}{ Analgesic } & & & L. garganicum & [19] & & & & & & & T. satureioides & \\
\hline & & & L. purpureum & [19] & & & & & & & T. maroccanus & [246] \\
\hline & & & & & & & & & & & T. leptobotrys & \\
\hline \multirow[t]{2}{*}{ Neuroprotective } & & & & & L. angustifolia & {$[248,249]$} & & & M. $\times$ piperita & [216] & & \\
\hline & & & & & L. viridis & [168] & & & M. aquatica & $\begin{array}{l}{[72,191,} \\
216]\end{array}$ & & \\
\hline \multirow[t]{3}{*}{ Tyrosinase inhibitor } & & & L. amplexicaule & [160] & L. stoechas & [115] & & & & & & \\
\hline & & & & & L. latifolia & {$[115,206]$} & & & & & & \\
\hline & & & & & L. allardii & [115] & & & & & & \\
\hline Anticonvulsant & & & & & L. stoechas & [250] & & & & & & \\
\hline Cardioprotective & & & & & & & L. sibiricus & [222] & & & T. pulegioides & [251] \\
\hline Anxiolytic & C. scoparius & [6] & & & & & & & & & & \\
\hline Hepatoprotective & C. scoparius & {$[4,6,98]$} & & & & & & & & & & \\
\hline
\end{tabular}


3. RESULTS AND DISCUSSION 
Regardless the exponential investigation on phenolic compounds in the last decades, the scientific knowledge on the phenolic composition of many plants, as well as on the mechanisms of action associated to their health benefits, remain far from being fully elucidated [255, 256]. In this context, the first aim of this Doctoral Thesis was to investigate the phenolic composition of the ethanolic extracts of the unexploited species Thymus $x$ citriodorus, Cytisus multiflorus and Lamium album L., Leonurus cardiaca L., Mentha aquatica L. and Lavandula dentata L., by means of the combined methods HPLC-DAD plus ESI-MS, MS ${ }^{n}$ and NMR. Further studies aimed to evaluate the antioxidant capacities of the extracts, as well as other biological properties that can add value to these plants.

The phenolic extracts of the six plants were obtained by extraction with an $80 \%$ ethanolic aqueous solution $(v / v)$, after defatting with $n$-hexane. Some of the ethanolic extracts were also purified on Strata SPE C18-E cartridges (2 g, Waters, Milford, MA, USA), for phenolic enrichment. The resulting extracts were further analyzed by reversed phase HPLC-UV, ESI-MS in the negative ion mode and MS $^{n}$ combined techniques. Moreover, NMR experiments were performed in order to confirm the exact structure of the major phenolic compounds of some plant extracts. The analysis of $T . x$ citriodorus extract was also validated concerning its linearity, instrumental and method precision (for repeatability, immediate precision and intermediate precision) and accuracy (absolute recovery study).

The antioxidant activity was firstly estimated by chemical assays including 2,2diphenyl-2-picrylhydrazyl (DPPH•) scavenging and reducing power. The global toxicity of the extracts was evaluated in human hepatoblastoma HepG2 cells by the MTT test. In these cells, the protective effect of each extract $(50 \mu \mathrm{g} / \mathrm{mL})$ was evaluated through the measurement of the production of reactive oxygen species (ROS) in a model of oxidative stress with potassium dichromate (DK). Viability studies were performed in a similar model, after treating the cells with the extracts for 6 and $72 \mathrm{~h}$, allowing to determine their hepatoprotective effects. The cellular assays were also performed with individual phenolic constituents of the most promising extracts (apigenin, chrysin, eriodictyol, quercetin, luteolin, naringenin, rosmarinic acid and verbascoside) or with mixtures simulating the phenolic composition of the extracts at $50 \mu \mathrm{g} / \mathrm{mL}$. Hence, the individual contribution of phenolics of C. multiflorus, L. album, T. $x$ citriodorus on the beneficial effects herein investigated was also discussed in this section.

Besides these, other biological activities were investigated for C. multiflorus, $M$. aquatica and L. dentata extracts. Concretely, the scavenging activity of the $C$. multiflorus extract for two reactive species formed by immune system cells $(\mathrm{HOCl}$, 
$\mathrm{NO}^{\circ}$ ), and its inhibitory capacity on key inflammatory enzymes (5-LOX, iNOS and COX2) were assessed. Moreover, potential effects of $M$. aquatica and $L$. dentata extracts on bioenergetic functions of liver mitochondria was also evaluated through the measurement of respiratory parameters and transmembrane potencial. 


\subsection{SIMULTANEOUS CHARACTERIZATION AND QUANTIFICATION OF PHENOLIC COMPOUNDS IN THYMUS X CITRIODORUS USING A VALIDATED HPLC-UV AND ESI-MS COMBINED METHOD}

Thymus $x$ citriodorus, also known as lemon thyme, is a plant used for several cooking and medicinal purposes. Despite its wide use as tea or as flavor ingredient, the phenolic composition of this species is yet unknown.

The in-house validated HPLC-DAD method showed good linearity for the tested reference compounds as well as satisfactory repeatability and immediate precision values, for both instrument and method. Furthermore, the satisfactory results of intermediate precision analysis and recovery assays indicated that the chromatographic method could be used to quantify the main phenolic compounds of $T$. $x$ citriodorus with adequate precision and accuracy. The fractionation of the ethanolic extract by HPLC-DAD and the analysis of the collected fractions by ESI-MS ${ }^{n}$, allowed to identify thirteen phenolic compounds. Structural confirmation by NMR was also achieved for major compounds.

Similarly to other Thymus species, the T. $x$ citriodorus ethanolic extract was enriched in rosmarinic acid $(10.4 \pm 0.6 \mathrm{mg} / \mathrm{g}$ extract). However, the extract was also enriched in Thymus non-typical phenolics, including the luteolin-7- $O-\alpha$-glucuronide $(12 \pm 2 \mathrm{mg} / \mathrm{g}$ extract) and apigenin-7- $\beta$-O-glucuronide ( $9 \pm 2 \mathrm{mg} / \mathrm{g}$ extract). Moreover, derivatives of the flavones luteolin, chrysoeriol and apigenin, of the flavanones eriodictyol and naringenin and of the flavonol quercetagetin, were also present in the extract. Concretely, the combined thecniques allowed to detect, for the first time in this genus, one eriodictyol di-O-hexoside, one chrysoeriol-7- $\beta$-O-glucoside, one quercetagetindimethyl-ether-O-hexoside and a naringenin-O-hexoside. Overall, the present study emerges as an important contribution emphasizing the phenolic constituents of $T . x$ citriodorus species.

The results obtained in this section of the Doctoral Thesis have been used to write the manuscript entitled "Simultaneous characterization and quantification of phenolic compounds in Thymus $x$ citriodorus using a validated HPLC-UV and ESI-MS combined method" which is presently submitted for publication. 


\section{Simultaneous characterization and quantification of phenolic compounds in}

2 Thymus $x$ citriodorus using a validated HPLC-UV and ESI- MS combined

3 method

${ }^{a}$ CERNAS - Escola Superior Agrária, Instituto Politécnico de Coimbra, Bencanta, 3040-316 Coimbra, Portugal;

${ }^{b}$ DTDT, Escola Superior de Saúde, Instituto Politécnico de Bragança, Av. D. Afonso V, 5300-121 Bragança, Portugal;

${ }^{c}$ CIMO,${ }^{d}$ LSRE - Escola Superior Agrária, Instituto Politécnico de Bragança, Campus de Santa Apolónia, 5301-854,

10 Bragança, Portugal;

$11{ }^{e}$ Departamento de Química \& QOPNA, Universidade de Aveiro, 3810-193 Aveiro, Portugal;

12

* Corresponding author (telephone: +351 239 802940; fax: +351 239 802979; e-mail:

14 scardoso@esac.pt).

15

16

17

Running title: Phenolic compounds in Thymus $x$ citriodorus.

18 


\section{Abstract}

24 Thymus $x$ citriodorus is a Lamiaceae plant extensively cultivated in Mediterranean region and used

25 for centuries in culinary and in traditional medicine. The present work describes the detailed

26 phenolic composition of T. $x$ citriodorus for the first time, the by means of HPLC-DAD, ESI-MS

27 and $\mathrm{MS}^{\mathrm{n}}$ and nuclear magnetic resonance (NMR) analyses. The ethanolic extract of T. $x$ citriodorus

28 was analyzed by reversed phase HPLC. The method of analysis was also validated concerning its

29 linearity, instrumental and method precision (for repeatability, immediate precision and intermediate

30 precision) and accuracy (absolute recovery study). The technique was combined with electrospray

31 mass spectrometry in order to identify the phenolic compounds and the structure of the main

32 phenolics was also confirmed by NMR analysis. The in-house validated HPLC-DAD method

33 showed good linearity for the tested reference compounds as well as satisfactory repeatability and

34 immediate precision values, for both instrument and method. Furthermore, the satisfactory results of

35 intermediate precision analysis and recovery assays indicated that the chromatographic method

36 could be used to quantify the main phenolic compounds of T. $x$ citriodorus with adequate precision

37 and accuracy. The extract was rich in rosmarinic acid $(10.4 \pm 0.6 \mathrm{mg} / \mathrm{g}$ extract) that is a widespread

38 phenolic acid in Thymus plants, but also in luteolin-7-O- $\alpha$-glucuronide $(12 \pm 2 \mathrm{mg} / \mathrm{g}$ extract), that was

39 herein reported in Thymus for the first time. Other novel compounds comprised one eriodictyol

40 dihexoside with $O$-glycosidic linkages, two eriodictyol- $O$-monohexosides, one quercetagetin

41 dimethyl ether- $O$-hexoside, one naringenin- $O$-hexoside and chrysoeriol-7- $\beta-O$-glucoside. Having in

42 mind the health-promoting properties reported in literature for some of the main polyphenols found

43 in T. $x$ citriodorus, we suggest that this plant has a high potential for being used as a functional food. 
45 Keywords: Thymus $x$ citriodorus; phenolic compounds; flavonoids; luteolin-7- $O$ - $\alpha$-glucuronide;

46 rosmarinic acid; mass spectrometry; electrospray ionization. 


\section{Introduction}

49 In recent years, several industries have shown a great interest in edible plants and in their bioactive

50 compounds because of their potential applications, including as functional food and nutraceuticals

51 (Wijngaard, Hossain, Rai, \& Brunton, 2012). Thymus L. is a large genus belonging to the Lamiaceae

52 family, which comprises 300-400 endemic species widely distributed in the entire World, in

53 particular in the Mediterranean region. These plants are perennial, herbaceous, tender and of simple

54 small leaves with ramified and prostrated branches, forming a shrub with uncountable branches,

55 typically reaching a height of 10 to $30 \mathrm{~cm}$ (Reddy, Angers, Gosselin, \& Arul, 1998).

56 Many Thymus species are known as culinary herbs and have been cultivated for usage in the 57 confection of several dishes and in flavoring salads, soups, stews and sauces. Additionally, Thymus

58 species are used in infusion form as medicinal plants because of their biological and

59 pharmacological properties, which include expectorant, anti-asthmatic, bronchiolytic, anti-septic,

60 antispasmodic, analgesic, antimicrobial, and antioxidant (Gião et al., 2007; Mata et al., 2007; Pinto

61 et al., 2006). It is believed that some of these beneficial activities are due to their volatile

62 constituents and thus, their essential oil composition has been the focus of many investigations

63 (Horvath, Szabo, Hethelyi, \& Lemberkovics, 2006; Omidbaigi, Sefidkon, \& Hejazi, 2005). In

64 contrast, there is only a limited number of studies focusing the composition of other bioactive 65 phytochemicals of Thymus plants, such as their phenolic compounds. According to the few studies

66 on this topic, the hydrophilic extracts of dried thyme plants contain caffeic acid and its oligomers

67 [rosmarinic acid, 3'-O-(8''-caffeoyl)rosmarinic acid, lithospermic acid and methyl rosmarinate],

68 flavones (apigenin, luteolin, luteolin-7-O- $\beta$-glucuronide, luteolin-7-O-glucoside, 6-hydroxyluteolin

69 glycosides, chrysoeriol and polymethoxyflavones), flavanones (naringenin, naringenin-7-O-

70 glucoside, narirutin, eriodictyol, eriodictyol-7- $O$-glucoside, isosakuranetin, eriocitrin and 
71 hesperidin), and the flavanol taxifolin (Dorman, Bachmayer, Kosar, \& Hiltunen, 2004; Fecka \&

72 Turek, 2008).

73 Thymus $x$ citriodorus, or lemon thyme, is one of the most used Thymus in culinary (The Herb

74 Society of America, 2003). The plant is used as an ingredient for confection of several recipes of 75 starter (Cheese-stuffed Nasturtiums), snacks, sauces (Chilli Oil, Soyer's Recipe for Goose Stuffing) 76 and different meat (Meat Stuffing for Duck), fish (Fish Aspic Jelly) or vegetarian (Spinach Frittata 77 with Herbs) dishes. Additionally, it is used in jellies and desserts (Lemon Thyme Jelly) for 78 confection of soups (Cream of Porcini Soup, Thick Giblet Soup) and consumed in fresh salads as 79 well as in marinades for grilled fish, chicken and roast duck, potatos and carrots (Celtnet, 2013). 80 Besides its culinary usage, $T$. $x$ citriodorus is also vastly consumed in the form of tea, for medicinal 81 proposals.

82 Despite the widespread culinary consumption of T. $x$ citriodorus and its claimed health benefits, the 83 detailed knowledge of its phenolic constituents remains unknown. The present work used a 84 combination of HPLC with ESI-MS/MS ${ }^{\mathrm{n}}$ and nuclear magnetic resonance (NMR) analysis, in order 85 to contribute for the knowledge of the phenolic constituents in T. x citriodorus. 


\section{2. Experimental}

\subsection{Plant material}

91 T. $x$ citriodorus plants were purchased from ERVITAL (Castro de Aire, Portugal) as a mixture of

92 leaves and stems. The plants have been cultivated under an organic regime and the collected aerial

93 parts were dried at $25-30^{\circ} \mathrm{C}$ in a ventilated incubator for approximately 5 days.

\subsection{Solvents and reagents}

96 -Hexane was purchased from Pronalab (Lisbon, Portugal), the analytical grade reagents formic acid

97 and ethanol were obtained from Panreac (Barcelona, Spain), methanol and acetonitrile with HPLC 98 purity were purchased from Lab-Scan (Lisbon, Portugal). Water was treated in a Mili-Q water 99 purification system (TGI Pure Water Systems, USA). Eriodictyol-7- $O$-glucoside, luteolin-7- $O$ 100 glucoside, naringenin-7-O-glucoside, apigenin-7-O-glucoside, chrysoeriol and rosmarinic acid were 101 obtained from Extrasynthese (Genay Cedex, France).

102

103

\subsection{Extraction of phenolic compounds}

104 The phenolic extract of the aerial parts of T. $x$ citriodorus was obtained by extraction with an $80 \%$ 105 ethanolic aqueous solution (v/v), after defatted with $n$-hexane, as previously described (Pereira, 106 Domingues, Silva, \& Cardoso, 2012) These procedures were performed in triplicate. 


\subsection{HPLC-DAD analyses}

\section{$112 \quad$ 2.4.1. HPLC apparatus and chromatographic conditions}

113 The HPLC analysis was performed on a Varian 9010 separation module equipped with PDA Varian

114 Prostar detector. Data acquisition and remote control of the HPLC system were done by Varian Star 115 chromatography Workstation ${ }^{\circledR}$ (Lake Forest, CA, USA) software. The column was a $250 \mathrm{~mm} \times 4$ $116 \mathrm{~mm}$ id, $5 \mu \mathrm{m}$ bead diameter, end-capped Nucleosil C18 (Macherey-Nagel) and its temperature was 117 maintained at $30^{\circ} \mathrm{C}$.

118 Gradient elution was carried out with a mixture of two solvents. Solvent A consisted of $0.1 \%(\mathrm{v} / \mathrm{v})$ 119 of formic acid in water and solvent B consisted of acetonitrile, which were degassed and filtrated, 120 using a $0.2 \mu \mathrm{m}$ nylon filter (Whatman, USA) before use. The solvent gradient consisted in a series of 121 linear gradients: from 10 to $30 \%$ of solvent B over $20 \mathrm{~min}$, from 30 to $100 \%$ of solvent B over 5 $122 \mathrm{~min}$, decreasing to $10 \%$ of solvent $\mathrm{B}$ after $5 \mathrm{~min}$ followed by the return to the initial conditions. The 123 flow rate was $1 \mathrm{~mL} / \mathrm{min}$ and the injected volume was equal to $10 \mu \mathrm{L}$.

\subsubsection{Method in-house validation}

126 The HPLC method used to detect and quantify the phenolic compounds was validated for linearity, 127 precision (assays performed for repeatability and intermediate precision) and accuracy (absolute 128 recovery study).

129 Linearity, limits of detection and of quantification. An external standard calibration methodology 130 was applied. Five solutions with different concentrations of eriodictyol-7-O-glucoside (10.0-135.9 $131 \mu \mathrm{g} / \mathrm{mL})$, naringenin-7-O-glucoside $(5.0-67.9 \mu \mathrm{g} / \mathrm{mL})$, luteolin-7-O-glucoside $(45.3-300.0 \mu \mathrm{g} / \mathrm{mL})$, 132 apigenin-7-O-glucoside $(2.5-160.0 \mu \mathrm{g} / \mathrm{mL})$ and rosmarinic acid $(14.9-120.0 \mu \mathrm{g} / \mathrm{mL})$ were prepared 133 by consecutive dilutions from a stock solution. The analyses were performed in triplicate and the 134 results were plotted for evaluating the linear relationship between the peak areas of each phenolic 
135 standard. ANOVA was used to assess the statistical significance of each linear regression model was 136 being the quality of the fitted models evaluated by their $\mathrm{R}^{2}$ values. The statistical significances of the 137 slope and of the intercept values were evaluated by a t-test. Finally, the regression data were 138 subjected to a likelihood ratio test of equality (covariance analysis) to infer about inter-day 139 variability of the calibration curves in order to avoid establishing a new calibration curve whenever 140 a quantification procedure was needed. Statistic analyses were performed using the SPSS 17 141 Standard Version software (SPSS INC.) at a 5\% significance level. Detection (LOD) and 142 quantification (LOQ) limits were calculated using the parameters of the calibration curves, being 143 defined as 3.3 and 10 times the value of the regression error divided by the slope, respectively 144 (Ermer \& Miller, 2005; Snyder, Kirkland, \& Dolan, 2010).

145 Precision (repeatability and intermediate precision). Both instrumental and method precisions were 146 evaluated to verify the repeatability of the system and of the proposed method (extraction procedure 147 followed by chromatographic analysis).

148 The instrumental system precision was studied using three standard solutions, containing 149 eriodictyol-7- $O$-glucoside $(10,30$ or $80 \mu \mathrm{g} / \mathrm{mL})$, naringenin-7- $O$-glucoside $(5,40$ or $50 \mu \mathrm{g} / \mathrm{mL})$, 150 luteolin-7-O-glucoside $(45,100$ or $300 \mu \mathrm{g} / \mathrm{mL})$, apigenin-7- $O$-glucoside $(10,80$ or $160 \mu \mathrm{g} / \mathrm{mL})$, and 151 rosmarinic acid $(15,30$ or $150 \mu \mathrm{g} / \mathrm{mL})$, which corresponded to low, middle and high concentration 152 levels. Each solution was injected, under the working conditions, 5 times on the same day to 153 evaluate the repeatability of the instrumental system (i.e., intra-day variation, considering only 154 within day variations). Further, the immediate precision of the system was evaluated by determining 155 the variability of the responses of the injections of the three standard solutions, injected 3 times per 156 day in three consecutive days (i.e., inter-day variation, considering within and between day 157 variations). The instrumental precision was assessed by calculating the relative standard deviation 158 percentage (\% RSD). 
159 The method precision was inferred based on the evaluation of repeatability and immediate precision.

160 For that, an ethanolic extract from T. $x$ citriodorus was obtained according to the work conditions, 161 from a sample of $T . x$ citriodorus $(5.0010 \pm 0.0001 \mathrm{~g})$ resulting in $0.75 \mathrm{~g}$ of lyophilized extract. Part 162 of the lyophilized extract $(50.6 \mathrm{mg})$ was re-dissolved into $5 \mathrm{~mL}$ of methanol and then injected 5 163 times in the same day and 3 times per day in three consecutive days for method repeatability and 164 immediate precision assessment, respectively.

165 The intermediate precision of the method was studied using two extracts obtained from the same 166 plant according to the procedure previously described (extracted, stirred, filtered, re-extracted, 167 combined, concentrated under reduced pressure, frozen and finally freeze-dried separately). Each 168 extract was injected in triplicate in three consecutive days. Subsequently, the intermediate precision

169 of the method was evaluated by calculating the \% RDS value of each phenolic compound detected, 170 considering within and between day variations as well as between extraction variations.

171 Accuracy. The accuracy of the proposed method was studied by evaluating the absolute recovery, 172 which studies the retrieval of standards added to a biological sample, that was subjected to all steps 173 of the extraction procedure (extraction, filtration, re-extraction, concentration and freeze-dried). Two 174 levels of two available phenolic standards corresponding to natural phenolic constituents of the T. $x$ 175 citriodorus were added to $2.04 \pm 0.02 \mathrm{~g}$ of $T . x$ citriodorus dry plant samples. The quantity of each 176 substance recovered in relation to the added amount was calculated, taking into account the yield of 177 the extraction procedure.

\subsubsection{Identification and quantification of the phenolic compounds}

180 The identification of the phenolic compounds of the ethanolic extracts of T. $x$ citriodorus was based 181 on the UV-Vis spectrum of the HPLC fractions together with their analysis by electrospray 182 ionization mass spectrometry (ESI-MS and ESI-MS $\left.{ }^{\mathrm{n}}\right)$. This latter was performed on a Linear Ion 
183 trap LXQ mass spectrometer (ThermoFinnigan, San Jose, CA, USA), following the procedure 184 previously described (Pereira, Silva, Domingues, \& Cardoso et al., 2012). When standards were 185 available, the identification of the compounds was further confirmed by comparison of their HPLC186 DAD retention time, UV-Vis profile and ESI-MS ${ }^{\mathrm{n}}$ data to those of the phenolic standards. 187 Moreover, the structure of some compounds (luteolin-5- $\beta-O$-glucoside, luteolin-7- $\alpha-O$-glucuronide, 188 chrysoeriol-7- $\beta$ - $O$-glucoside, apigenin-7- $\beta$ - $O$-glucuronide and rosmarinic acid) was further 189 confirmed by NMR analysis. The dried HPLC-collected fractions were dissolved in DMSO- $\mathrm{d}_{6}$ and 190 the ${ }^{1} \mathrm{H}$ spectra were recorded at $298 \mathrm{~K}$ on a Bruker Avance 500 spectrometer operating at 500.13 191 MHz. 2D NMR (heteronuclear single quantum coherence, using gradient pulses for selection i.e. $192\left({ }^{1} \mathrm{H},{ }^{13} \mathrm{C}\right) \mathrm{gHSQC}$, heteronuclear multiple quantum coherence, using gradient pulses for selection i.e. $193 \mathrm{gHMBC}$ ) spectra were acquired in the same experimental conditions as previously described 194 (Pereira, et al., 2012a). ${ }^{13} \mathrm{C}$ NMR chemical shift assignments were made from the projections of the 195 heteronuclear HSQC and HMBC experiments.

196 The quantification of phenolic compounds was performed by peak integration using the external 197 standard method, with the most close reference compound available to that of the major compound 198 in each HPLC eluting peak. 


\section{Results and discussion}

\subsection{Identification of the phenolic compounds of the ethanolic extract of $T$. $x$ citriodorus}

204 rosmarinic acid and another less common phenolic acids, as well as derivatives of common 205 flavonoids, including the flavones luteolin, chrysoeriol and apigenin, the flavanones eriodictyol and 206 naringenin, and the flavonol quercetagetin (Table 1). A description of the T. x citriodorus phenolic 207 composition and the comparison to that previous described for other Thymus plants, will be 208 discussed in bellow in detail.

\subsubsection{Phenolic Acid derivatives}

211 In accordance with literature data, the rosmarinic acid, which was herein identified by its retention 212 time, UV-Vis spectrum, ESI-MS ${ }^{\mathrm{n}}$ (Table 1) and NMR (Table 2) data, represented a major HPLC 213 fraction in the T. $x$ citriodorus ethanolic extract (fraction 9, Fig.1). To our knowledge, this 214 compound has been previously detected in T. serpyllum, T. sipyleus, T. quinquecostatus and T. 215 vulgaris L., and has been shown to account between 3.4 to $22 \mathrm{mg} / \mathrm{g}$ of dry plant, in the latter species 216 (Pereira \& Cardoso, 2013). Besides this phenolic acid, the T. $x$ citriodorus ethanolic extract also 217 contained an uncommon caffeoyl derivative of rosmarinic acid, which was assigned based on its 218 retention time and UV-Vis spectrum, as compared to that of rosmarinic acid, plus interpretation of 219 its ESI-MS ${ }^{\mathrm{n}}$ fragmentation pattern (Dapkevicius et al., 2002). Most probably, this compound 220 corresponds to $3^{\prime}-O-\left(8^{\prime \prime}-Z\right.$-caffeoyl)rosmarinic acid, which has been previously detected in the 221 leaves of T. vulgaris (Dapkevicius et al., 2002). 


\subsubsection{Flavones}

224 Flavones were also detected as major phenolic component of the T. $x$ citriodorus ethanolic extract. 225 In more detail, the extract contained three luteolin derivatives, which were eluted in fractions 4 and $6\left([\mathrm{M}-\mathrm{H}]^{-}\right.$ion at $m / z, 447$ in fraction 4 and $[\mathrm{M}-\mathrm{H}]^{-}$ions at $\mathrm{m} / \mathrm{z}, 461$ and $\mathrm{m} / \mathrm{z} 447$ in fraction 6). The $227[\mathrm{M}-\mathrm{H}]^{-}$ion at $\mathrm{m} / \mathrm{z} 447$ in the latter fraction was assigned to luteolin-7- $O$-glucoside, since the retention time and $\mathrm{MS}^{\mathrm{n}}$ data matched with those of the reference compound. In turn, the $[\mathrm{M}-\mathrm{H}]^{-}$ion at $m / z, 447$ in fraction 4 was assigned to luteolin-5- $\beta-O$-glucoside, based on the gathered 1D (Table

2) and $2 \mathrm{D}$ NMR spectral data. From these spectra it was possible to assign the major part of the ${ }^{1} \mathrm{H}$ 231 and ${ }^{13} \mathrm{C}$ resonances, mainly obtained from the connectivities found in the HMBC spectrum of this 232 compound (the connectivity between $\mathrm{H}-1$ ", and C-5 allowed us to assign the sugar position on the 233 flavone skeleton). The coupling constant of the $\mathrm{H}-1$ of the sugar moiety $(J=7.3 \mathrm{~Hz})$ indicates the 234 presence of the $\beta$-anomer. To our knowledge, for Thymus plants the latter luteolin derivative has only been previously detected in T. sipyleus and in T. praecox (Ozgen et al., 2011).

236 Moreover, the HPLC-DAD-ESI-MS ${ }^{\mathrm{n}}$ analysis allowed to assign the $[\mathrm{M}-\mathrm{H}]^{-}$ion at $\mathrm{m} / \mathrm{z}, 461$ in 237 fraction 6 to luteolin- $O$-hexuronyl, as the product ion at $m / z 285$ was obtained by the loss of 176 Da. 238 Additional information on the sugar moiety (glucuronic acid), as well as its linkage position on the 239 flavone skeleton (7-) was elucidated by 1D and 2D NMR analysis. These NMR spectra allowed us 240 to assign the major part of the proton and carbon resonances, but due to the small quantity of the 241 sample we could not found the HMBC correlation between $\mathrm{H}-1$ ', from the sugar residue and the C-7 242 of the flavone. However, the assigned chemical shifts are compatible with a 7-glucuronide (Agrawal 243 \& Bansal, 1989; Lu \& Foo, 1999). H-1"' of the sugar residue appear as a broad singlet, which is 244 only compatible with an $\alpha$-configuration. Note that despite luteolin- $O$-glucuronide has been 245 previously described in several Thymus plants (Justesen, 2000; Miron, Plaza, Bahrim, Ibanez, \& 
Herrero, 2011), only the 7-O- $\beta$-isomer has been previously identified by NMR analysis (Fecka \&

247 Turek, 2008; Ozgen et al., 2011).

248 Besides the luteolin derivatives, the T. $x$ citriodorus ethanolic extract also contained another flavone 249 hexuronyl derivative (fraction $8,[\mathrm{M}-\mathrm{H}]^{-}$ion at $\mathrm{m} / \mathrm{z} 445$ ). In this particular case, the $\mathrm{MS}^{2}$ spectrum 250 showed a main ion at $\mathrm{m} / z, 269$, and the latter followed a fragmentation pattern consistent to that of 251 apigenin. According to the NMR determinations (Guvenalp, Ozbek, Kuruuzum-Uz, Kazaz, \& 252 Demirezer, 2009) the $[\mathrm{M}-\mathrm{H}]^{-}$ion at $m / z 445$ was assigned to apigenin-7- $\beta-O$-glucuronide, which, for 253 the Thymus genus, has only been previously reported in T. vulgaris and T. serpyllum (Justesen, 254 2000; Miron et al., 2011).

255 Notably our study also allowed to detect, for the first time in Thymus plants, an hexoside derivative 256 of the methylated flavone chrysoeriol. This compound was eluted in fraction 7 and appeared in the 257 ESI-MS spectrum as the $[\mathrm{M}-\mathrm{H}]^{-}$ion at $\mathrm{m} / \mathrm{z} 461$ that fragmented to a main product ion at $\mathrm{m} / \mathrm{z} 299(-$ $258162 \mathrm{Da}$, loss of hexose). Moreover, the fragmentation pattern of this product ion was similar to that 259 of chrysoeriol (Plazonic et al., 2009). This information was corroborated by the NMR analysis, 260 which also allowed to assign the exact structure of this flavone to chrysoeriol-7- $\beta$ - $O$-glucoside 261 (Table 2). The $\beta$-configuration of the sugar residue is based on the coupling of the $\mathrm{H}-1$ ',,$J=7.3 \mathrm{~Hz}$.

\subsubsection{Flavanones}

264 Three glycoside derivatives of eriodictyol have been previous described in the genus Thymus, 265 namely the eriodictyol-7-O-glucoside (Fecka \& Turek, 2008), the eriodictyol-7-O-rutinoside (Wang, $266 \mathrm{Li}, \mathrm{Ho}$, Peng, \& Ho, 1998) and the eriodictyol-7-O-glucuronide (Justesen, 2000). Nevertheless, the 267 results now obtained show that three of the four eriodictyol derivatives detected in the ethanolic 268 extract of T. $x$ citriodorus differ from those previously reported for the other species of Thymus. 269 Indeed, the ESI-MS analysis of fraction 1 showed the $[\mathrm{M}-\mathrm{H}]^{-}$ion at $m / z, 611$ and its $\mathrm{MS}^{2}$ and $\mathrm{MS}^{3}$ 
fragmentation data revealed the loss of two hexose molecules (product ions at $\mathrm{m} / \mathrm{z}, 449$ and $\mathrm{m} / \mathrm{z}$ 287).

271 Moreover, the ESI-MS ${ }^{3}$ spectrum of the $[\mathrm{M}-\mathrm{H}]^{-}$ion at $\mathrm{m} / \mathrm{z} 449$ was similar to that of authentic 272 eriodictyol-7-O-glucoside and hence, these data indicates the elution in fraction 1 of an eriodictyol 273 dihexoside with $O$-glycosyl linkages. Note that, flavonoid diglycosides with $O$-linkages (di- $O$ 274 glycosides and $O$-diglycosides), can be distinguished by their product ion spectra (Vukics \& 275 Guttman, 2008), through the analysis of the $[\mathrm{M}-\mathrm{H}-162]^{-},[\mathrm{M}-\mathrm{H}-180]^{-}$and $[\mathrm{M}-\mathrm{H}-324]^{-}$ions. 276 A high relative intense product ion $[\mathrm{M}-\mathrm{H}-162]^{-}$and the absence of $[\mathrm{M}-\mathrm{H}-180]^{-}$at the $\mathrm{MS}^{2}$ 277 spectrum, as observed for the ion at $\mathrm{m} / \mathrm{z} 611$, indicates the presence of a flavonoid di- $O$-hexoside 278 and thus, the present results confirm the detection, for the first time in Thymus, of an eriodictyol-di$279 O$-hexoside.

280 Eriodictyol-O-monohexosides were detected in fractions 2 and 3. As observed in Table 1, the ESI281 MS spectra of both fractions showed a $[\mathrm{M}-\mathrm{H}]^{-}$ion at $m / z 449$, and their $\mathrm{MS}^{2}$ spectrum revealed the 282 ion at $\mathrm{m} / \mathrm{z} 287$, which corresponds to the eriodictyol aglycone. The UV-Vis data of these two peaks 283 were similar to that of the eriodictyol-7-O-glucoside standard, but fractions 2 and 3 eluted before 284 that compound, indicating that two new eriodictyol- $O$-hexosides must be present in $T$. $x$ citriodorus. 285 In fact, to our knowledge, eriodictyol-7-O-glucoside is the only described glucoside derivative of 286 eriodictyol in Lamiaceae family. Besides the above described O-hexosyl derivatives of eriodictyol, 287 the T. $x$ citriodorus ethanolic extract also contained an $O$-hexuronyl of this flavanone, but this 288 appeared only as a minor constituent of fraction 5. Eriodictyol-O-glucuronide has been previously 289 found in thyme and wild thyme (Justesen, 2000; Miron et al., 2011), but no quantitative information 290 has been delivered on that compound on those plants. Besides eriodictyol derivatives, the flavavone 291 naringenin- $O$-hexoside was also identified as a phenolic constituent of the T. $x$ citriodorus ethanolic 292 extract. Its $[\mathrm{M}-\mathrm{H}]^{-}$ion was observed in fraction 5 at $\mathrm{m} / \mathrm{z}, 433$ and this fragmented to the ion at $\mathrm{m} / \mathrm{z}$ 293271 (-162 Da). Moreover, the $\mathrm{MS}^{3}$ spectrum of the ion at $\mathrm{m} / \mathrm{z} 271$ (main ions at $\mathrm{m} / \mathrm{z} 227,151$ and 
294 107), indicated a correspondence to authentic naringenin (Fabre, Rustan, de Hoffmann, \& Quetin295 Leclercq, 2001). Despite the detailed structure of this compound could not be accomplished in the 296 present study, for sure, this does not corresponds to the unique naringenin glucoside described so far 297 in Thymus, i.e. the naringenin-7-O-glucoside (Fecka \& Turek, 2008), as it eluted before the 298 naringenin-7-O-glucoside standard (10.5 min).

\subsubsection{Flavonols}

301 In accordance to the previous studies focusing on Thymus plants, the ethanolic extract of T. $x$ 302 citriodorus was very poor in flavonols. In the present study, the unique flavonol was detected as a 303 minor component of fraction 2. Its corresponding molecular ion was observed for $\mathrm{m} / 2,507$ and its $304 \mathrm{MS}^{2}$ spectrum showed product ions formed by the loss of a hexose moiety (ion at $\mathrm{m} / \mathrm{z} 345$ (-162 Da) 305 and ion at $\mathrm{m} / \mathrm{z} 327$ (simultaneous loss of 162 and $18 \mathrm{Da}$ )). Considering that the ion at $\mathrm{m} / \mathrm{z} 345 \mathrm{can}$ 306 diagnose the quercetagetin dimethyl ether (Parejo, Jauregui, Viladomat, Bastida, \& Codina, 2004), 307 the latter hypothesis also supported by its $\mathrm{MS}^{3}$ data that shows the ion at $\mathrm{m} / \mathrm{z} 315$ (- $30 \mathrm{Da}$, 308 equivalent to the loss of two methyl groups), the overall data suggests the presence of the flavonol 309 quercetagetin dimethyl ether- $O$-hexoside in T. $x$ citriodorus. Methyl derivatives of quercetagetin, 310 namely quercetagetin 3,7-dimethyl ether, have been described to occur in the Lamiaceae family

311 (Grayer et al., 2010), however this the first time that this class of compounds has been found in the 312 genus Thymus.

\section{3.2. HPLC method - validation and quantification of phenolic compounds by HPLC-DAD}

315 For the five used phenolic standards, the adjusted $\mathrm{R}^{2}$ values were around 0.999 , suggesting a good 316 linearity of the analytical method in the concentration range tested (Table 3) and showing that the 317 HPLC method allows the quantification of the evaluated phenolic compounds, in case of their 
318 presence in the plant samples. All the calibration linear models were significant $(\mathrm{P}<0.001)$ as well as 319 their slope values $(\mathrm{P}<0.001)$ and the intercept values $(\mathrm{P}<0.003$, except for apigenin- $O$-glucoside 320 with $\mathrm{P}=0.228$ ). Finally, the covariance analysis for each phenolic standard (data not shown) 321 indicated that the calibration curves of one week interval were not statistically different $(\mathrm{P} \geq 0.330)$, 322 meaning that the same calibration curve could be used during at least one week for quantification 323 purposes. Table 3 also shows the LOD and LOQ values, which were always lower than the lowest 324 standard concentration tested in the dynamic interval of the calibration curve, indicating a 325 satisfactory sensitivity towards each phenolic standard.

326 The relative standard deviation percentage (\%RSD) values of repeatability and of immediate 327 precision assays carried out with the five phenolic standards, regarding the instrumental precision 328 evaluation. In repeatability, \%RSD values varied from 2 to $5 \%$ and from 0.3 to $2 \%$, for the retention 329 time and the peak area, respectively. Concerning the immediate precision, the \%RSD values of the 330 retention times and peak areas were between $3-4 \%$ and $1-2 \%$, respectively. These results are similar 331 to those described by Du and co-workers (Du et al., 2010) and Gobbo-Neto and Lopes (2008) for 332 phenolic compounds in Lamiaceae and Asteraceae plants, respectively. Since the \%RSD values 333 were lower than $5 \%$, the chromatographic instrument presents a satisfactory precision (Ermer \& 334 Miller, 2005).

335 The \%RSD values for repeatability, immediate precision and intermediate precision assays of the 336 method (extraction plus HPLC analysis) are given in Table 3. As the values for the first two 337 parameters were lower than $12 \%$, it can be concluded that the precision of the method was 338 satisfactory. Regarding intermediate precision, the $\%$ RSD values varied from $6 \%$ to $41 \%$, being in 339 general lower than $20 \%$. The highest values (33\% and $41 \%$ for eriodictyol-7- $O$-glucoside and $3^{\prime}-O$ 340 (8"-Z-Caffeoyl)rosmarinic acid, respectively) could be due to the low content of these compounds 341 (Table 3), as well as to the nature of the extraction process and the complexity of the 
342 chromatographic analysis (Aldai, Osoro, Barrón, \& Nájera, 2006). In fact, depending on the samples 343 complexity, \%RSD values up to $20 \%$ are acceptable for quantitative chromatographic analysis of 344 residual analytes (Ribani, Bottoli, Collins, Jardim, \& Melo, 2004). Therefore, it can be stated that 345 the proposed method is able to quantify the major phenolic compounds detected in T. $x$ citriodorus 346 samples with overall satisfactory precision.

347 Regarding the two absolute recovery assays carried out (addition of $0.986 \mathrm{mg}$ of luteolin-7-O348 glucoside and $0.121 \mathrm{mg}$ of rosmarinic acid or $1.548 \mathrm{mg}$ of luteolin-7-O-glucoside and $0.555 \mathrm{mg}$ of 349 rosmarinic acid to $2.04 \pm 0.02 \mathrm{~g}$ of dry plant samples, respectively) recoveries were of $120 \%$ and $60 \%$ 350 or $121 \%$ and $79 \%$ for luteolin-7-O-glucoside and rosmarinic acid, respectively, with \%RSD values 351 between $2 \%$ and $4 \%$. These results are acceptable as, depending on the complexity of the 352 chromatographic method and the sample matrix, recovery values between $50 \%$ and $120 \%$ are 353 adequate if their \%RSD values are lower than 15\% (Ribani et al., 2004).

354 The mean total phenolic contents (mg/g of extract) of the T. $x$ citriodorus plant were evaluated from 355 the intermediate precision assays and are presented in Table 3. According to the results, the 356 identified phenolic compounds accounted for $44 \mathrm{mg}$ per $\mathrm{g}$ of the ethanolic extract (or $7.5 \mathrm{mg} / \mathrm{g}$ of 357 dried plant). Similarly to other Thymus species, such as T. vulgaris and T. spicata (Dorman et al., 358 2004; Fecka \& Turek, 2008), the ethanolic extract of T. $x$ citriodorus was mostly enriched in 359 rosmarinic acid $(10.4 \pm 0.6 \mathrm{mg} / \mathrm{g}$ extract). Still, our results also showed that in contract to the majority 360 of the previously studied Thymus plants, the non-common isomeric form of luteolin- $O$-hexoside, i.e. 361 the luteolin-7- $\alpha-O$-glucuronide, as well as apigenin-7- $\beta-O$-glucuronide, were also major phenolic 362 constituent of the extract, accounting for $12 \pm 2 \mathrm{mg} / \mathrm{g}$ extract and $9 \pm 2 \mathrm{mg} / \mathrm{g}$ extract, respectively. 


\section{Conclusions}

The phenolic composition of an ethanolic extract of T. $x$ citriodorus was described in detail

368 for the first time, by means of HPLC-DAD plus ESI-MS, MS ${ }^{\mathrm{n}}$ and NMR analysis. The in-house 369 validated HPLC-DAD method showed good linearity for the tested reference compounds as well as 370 satisfactory repeatability and immediate precision values, for both instrument and method. 371 Furthermore, the satisfactory results of intermediate precision analysis and recovery assays indicated 372 that the chromatographic method could be used to quantify the main phenolic compounds of $T . x$ 373 citriodorus with adequate precision and accuracy. The fractionation of the ethanolic extract by 374 HPLC-DAD and the analysis of the collected fractions by electrospray mass spectrometry in the 375 negative mode allowed to identify thirteen phenolic compounds, which include the phenolic acid 376 rosmarinic acid and one of its derivatives, as well as derivatives of the flavones luteolin, chrysoeriol 377 and apigenin, of the flavanones eriodictyol and naringenin and of the flavonol quercetagetin. 378 Similarly to other Thymus species, rosmarinic acid represented a major phenolic constituent of the $T$. $379 x$ citriodorus ethanolic extract. Whilst, our results also suggest that this plant species produces high 380 amounts of non-common Thymus phenolics, including the $7-\alpha-O$-glucuronide derivative of luteolin 381 and apigenin-7- $\beta-O$-glucuronide. As the major of these compounds are described in literature as 382 health-benefit compounds, we propose that this Thymus species can be used as nutraceutical agent 383 with potential interest for food and pharmaceutical industries. 


\section{Acknowledgements}

389 The authors gratefully acknowledge the financial support provided by the Foundation for Science 390 and Technology (FCT) to CERNAS (project PEst-OE/AGR/UI0681/2011) and of the FCT as well as 391 FSE (III Quadro Comunitário de Apoio), to QOPNA and REDE/1504/REM/2005 (that concerns the 392 Portuguese Mass Spectrometry Network) and the Portuguese National NMR Network (RNRMN).

393 Olívia R. Pereira was supported by a PhD grant (SFRH/PROTEC/49600/2009). Authors are also 394 grateful to Eng Jorge Sá Morais for technical support in the HPLC analysis.

$397 \quad$ Abbreviations used

398 CID - collision-induced dissociation; DAD - Diode array; ESI-MS - electrospray ionization-mass 399 spectrometry; $\mathrm{MS}^{\mathrm{n}}$ - tandem mass spectrometry; GAE - Gallic acid equivalent; HPLC - high400 performance liquid chromatography; LC - liquid chromatography; LOD - limit of detection; LOQ 401 limit of quantification; MS - Mass spectrometry; RSD - Relative standard deviation 402 403 


\section{References}

405 Agrawal, P. K., \& Bansal, M. C. (1989). Chapter 6: Flavonoid Glycosides In P. K. Agrawal (Ed.), Carbon-13 NMR of flavonoids (Vol. 28, pp. 283-364). Amsterdam: John Wiley \& Sons, Ltd.

Aldai, N., Osoro, K., Barrón, L. J. R., \& Nájera, A. I. (2006). Gas-liquid chromatographic method for analysing complex mixtures of fatty acids including conjugated linoleic acids (cis9trans11 and trans10cis12 isomers) and long-chain (n-3 or n-6) polyunsaturated fatty acids: Application to the intramuscular fat of beef meat. Journal of Chromatography A, 1110 (1-2), 133-139.

Celtnet. (2013). Celtnet Recipes. Retrieved from http://www.celtnet.org.uk/recipes/.

Dapkevicius, A., van Beek, T. A., Lelyveld, G. P., van Veldhuizen, A., de Groot, A., Linssen, J. P. H., \& Venskutonis, R. (2002). Isolation and structure elucidation of radical scavengers from Thymus vulgaris leaves. Journal of Natural Products, 65 (6), 892-896.

Dorman, H. J. D., Bachmayer, O., Kosar, M., \& Hiltunen, R. (2004). Antioxidant properties of aqueous extracts from selected Lamiaceae species grown in Turkey. Journal of Agricultural and Food Chemistry, 52 (4), 762-770.

Du, Y. F., Liu, P. W., Yuan, Z. F., Jin, Y. R., Zhang, X. W., Sheng, X. N., Shi, X. W., Wang, Q., \& Zhang, L. T. (2010). Simultaneous qualitative and quantitative analysis of 28 components in Isodon rubescens by HPLC-ESI-MS/MS. Journal of Separation Science, 33 (4-5), 545-557.

Ermer, J., \& Miller, J. M. (2005). Method Validation in Pharmaceutical Analysis: A Guide to Best Practice: Weinheim: WILEY-VCH Verlag GmbH \& Co.

Fabre, N., Rustan, I., de Hoffmann, E., \& Quetin-Leclercq, J. (2001). Determination of flavone, flavonol, and flavanone aglycones by negative ion liquid chromatography electrospray ion trap mass spectrometry. Journal of the American Society for Mass Spectrometry, 12 (6), 707 715. 
428 Fecka, I., \& Turek, S. (2008). Determination of polyphenolic compounds in commercial herbal drugs and spices from Lamiaceae: thyme, wild thyme and sweet marjoram by chromatographic techniques. Food Chemistry, 108 (3), 1039-1053.

431 Gião, M. S., Gonzalez-Sanjose, M. L., Rivero-Perez, M. D., Pereira, C. I., Pintado, M. E., \& 432

447 Mata, A. T., Proenca, C., Ferreira, A. R., Serralheiro, M. L. M., Nogueira, J. M. F., \& Araujo, M. E. Malcata, F. X. (2007). Infusions of Portuguese medicinal plants: Dependence of final antioxidant capacity and phenol content on extraction features. Journal of the Science of Food and Agriculture, 87 (14), 2638-2647.

Grayer, Eckert, Lever, A., Veitch, Kite, \& Paton. (2010). Distribution of exudate flavonoids in the genus Plectranthus. Biochemical Systematics and Ecology, 38 (3), 335-341.

Guvenalp, Z., Ozbek, H., Kuruuzum-Uz, A., Kazaz, C., \& Demirezer, L. O. (2009). Secondary metabolites from Nepeta heliotropifolia. Turkish Journal of Chemistry, 33 (5), 667-675.

Horvath, G., Szabo, L. G., Hethelyi, E., \& Lemberkovics, E. (2006). Essential oil composition of three cultivated Thymus chemotypes from Hungary. Journal of Essential Oil Research, 18 (3), 315-317.

Justesen, U. (2000). Negative atmospheric pressure chemical ionisation low-energy collision activation mass spectrometry for the characterisation of flavonoids in extracts of fresh herbs. Journal of Chromatography A, 902 (2), 369-379.

Lu, Y., \& Foo, L. Y. (1999). Rosmarinic acid derivatives from Salvia officinalis. Phytochemistry, 51 (1), 91-94. M. (2007). Antioxidant and antiacetylcholinesterase activities of five plants used as Portuguese food spices. Food Chemistry, 103 (3), 778-786. 
Miron, T. L., Plaza, M., Bahrim, G., Ibanez, E., \& Herrero, M. (2011). Chemical composition of bioactive pressurized extracts of Romanian aromatic plants. Journal of Chromatography A, $1218(30), 4918-4927$.

Omidbaigi, R., Sefidkon, F., \& Hejazi, M. (2005). Essential oil composition of Thymus citriodorus L. cultivated in Iran. Flavour and Fragrance Journal, 20 (2), 237-238.

Ozgen, U., Mavi, A., Terzi, Z., Kazaz, C., Asci, A., Kaya, Y., \& Secen, H. (2011). Relationship Between Chemical Structure and Antioxidant Activity of Luteolin and Its Glycosides Isolated from Thymus sipyleus subsp sipyleus var. sipyleus. Records of Natural Products, 5 (1), $12-21$.

Parejo, I., Jauregui, O., Viladomat, F., Bastida, J., \& Codina, C. (2004). Characterization of acylated flavonoid-O-glycosides and methoxylated flavonoids from Tagetes maxima by liquid chromatography coupled to electrospray ionization tandem mass spectrometry. Rapid Communications in Mass Spectrometry, 18 (23), 2801-2810.

Pereira, O. R., \& Cardoso, S. M. (2013). Overview on Mentha and Thymus Polyphenols Current Analytical Chemistry, 9 (3), 382-396.

Pereira, O. R., Domingues, M. R. M., Silva, A. M. S., \& Cardoso, S. M. (2012a). Phenolic constituents of Lamium album: Focus on isoscutellarein derivatives. Food Research International, 48 (1), 330-335.

Pereira, O. R., Silva, A. M. S., Domingues, M. R. M., \& Cardoso, S. M. (2012b). Identification of phenolic constituents of Cytisus multiflorus. Food Chemistry, 131 (2), 652-659.

Pinto, E., Pina-Vaz, C., Salgueiro, L., Goncalves, M. J., Costa-de-Oliveira, S., Cavaleiro, C., Palmeira, A., Rodrigues, A., \& Martinez-De-Oliveira, J. (2006). Antifungal activity of the essential oil of Thymus pulegioides on Candida, Aspergillus and dermatophyte species. Journal of Medical Microbiology, 55 (10), 1367-1373. 
474 Plazonic, A., Bucar, F., Males, Z., Mornar, A., Nigovic, B., \& Kujundzic, N. (2009). Identification 475 and Quantification of Flavonoids and Phenolic Acids in Burr Parsley (Caucalis platycarpos 476 L.), Using High-Performance Liquid Chromatography with Diode Array Detection and 477 Electrospray Ionization Mass Spectrometry. Molecules, 14 (7), 2466-2490.

478 Reddy, M. V. B., Angers, P., Gosselin, A., \& Arul, J. (1998). Characterization and use of essential 479 oil from Thymus vulgaris against Botrytis cinerea and Rhizopus stolonifer in strawberry $480 \quad$ fruits. Phytochemistry, 47 (8), 1515-1520.

481 Ribani, M., Bottoli, C. B. G., Collins, C. H., Jardim, I., \& Melo, L. F. C. (2004). Validation for 482 chromatographic and electrophoretic methods. Quimica Nova, 27 (5), 771-780.

483 Snyder, L., Kirkland, J., \& Dolan, J. (2010). Introduction to Modern Liquid Chromatography. New 484 Jersey: John Wiley \& Sons, Inc.

The Herb Society of America. (2003). An Herb Society of America Fact Sheet: Thyme. Retrieved from http://www.herbsociety.org/.

Vukics, V., \& Guttman, A. (2008). Structural characterization of flavonoid glycosides by multistage mass spectrometry. Mass Spectrometry Reviews, 29 (1), 1-16.

Wang, M., Li, J., Ho, G. S., Peng, X., \& Ho, C. T. (1998). Isolation and identification of antioxidative flavonoid glycosides from thyme (Thymus vulgaris L.). Journal of Food Lipids, 5 (4), 313-321.

Wijngaard, H., Hossain, M. B., Rai, D. K., \& Brunton, N. (2012). Techniques to extract bioactive compounds from food by-products of plant origin. Food Research International, 46 (2), 505 - 


\section{$497 \quad$ Figure Captions}

498

499 Fig.1. Chromatographic profile of the ethanolic extract obtained from T. $x$ citriodorus. The numbers

500 in the figure correspond to the fractions collected for further analysis by ESI-MS ${ }^{\mathrm{n}}$ and NMR.

501 


\begin{tabular}{|c|c|c|c|c|c|}
\hline $\begin{array}{l}\text { Fraction } \\
\text { number }\end{array}$ & $\begin{array}{c}\mathbf{R T} \\
(\mathbf{m i n})\end{array}$ & $\lambda_{\max }$ & {$[\mathbf{M}-\mathbf{H}]^{-}$} & $\begin{array}{c}\text { Main } \\
\text { ESI }^{-} \mathbf{M S}^{\mathbf{n}} \\
\text { (Abundance) } \\
\end{array}$ & Compound \\
\hline 1 & 4.3 & 283,327 & 611 & $\begin{array}{l}\mathrm{MS}^{2}[611]: \text { 449(100), 287(15); } \mathrm{MS}^{3}[449]: \\
\text { 287(100), 151(<1); } \mathrm{MS}^{4}[287]: 269(2), \\
\text { 151(100); } \mathrm{MS}^{5}[151]: 107\end{array}$ & Eriodictyol-di- $O$-hexoside \\
\hline \multirow{3}{*}{2} & \multirow{3}{*}{6.8} & \multirow{3}{*}{283,327} & 387 & $\begin{array}{l}\operatorname{MS}^{2}[387]: \text { 369(15), 225(5), 207(100), } \\
\text { 163(10), 119(1); } \mathrm{MS}^{3}[207]: 163 \\
\operatorname{MS}^{4}[163]: 109\end{array}$ & 5'-Hydroxyjasmonic acid 5'-O-hexoside \\
\hline & & & 449 & $\begin{array}{l}\operatorname{MS}^{2}[449]: 287 ; \operatorname{MS}^{3}[287]: 151 \\
\operatorname{MS}^{4}[151]: 107\end{array}$ & Eriodictyol- $O$-hexoside \\
\hline & & & 507 & $\begin{array}{l}\operatorname{MS}^{2}[507]: 489(20), 471(10), 345(35), \\
\text { 327(100), 315(5); } \operatorname{MS}^{3}[327]: 312(100), \\
\text { 167(20); } \mathrm{MS}^{3}[345]: 327(100), 315(15), \\
\text { 309(20), 287(5) }\end{array}$ & Quercetagetin dimethyl ether $O$-hexoside \\
\hline 3 & 7.3 & 283,327 & 449 & $\begin{array}{l}\operatorname{MS}^{2}[449]: \text { 287(100), 269(<1), 151(1); } \\
\operatorname{MS}^{3}[287]: 269(4), 161(<1), 151(100) \\
\text { 125(4), 107(1); } \mathrm{MS}^{4}[151]: 107\end{array}$ & Eriodictyol- $O$-hexoside \\
\hline 4 & 8.6 & 248,342 & 447 & $\begin{array}{l}\operatorname{MS}^{2}[447]: \text { 285(100); } \operatorname{MS}^{3}[285]: 243(60) \\
241(100), 199(100), 175(50), 151(10)\end{array}$ & Luteolin-5- $O-\beta$-glucoside \\
\hline 5 & 9.1 & 283,340 & 433 & $\begin{array}{l}\operatorname{MS}^{2}[433]: \text { 271(100); } \mathrm{MS}^{3}[271]: 227(1) \\
\text { 177(10), 151(100), 107(2) }\end{array}$ & Naringenin- $O$-hexoside \\
\hline \multirow{3}{*}{6} & \multirow{3}{*}{9.6} & 283,327 & 463 & $\begin{array}{l}\operatorname{MS}^{2}[463]: 301(20), 287(100) ; \mathrm{MS}^{3}[287]: \\
\text { 151(100), 135(<1), 125(<1) }\end{array}$ & Eriodictyol- $O$-hexuronide \\
\hline & & \multirow[t]{2}{*}{$\begin{array}{c}254,267 \\
345\end{array}$} & 461 & $\begin{array}{l}\mathrm{MS}^{2}[461]: \text { 285(100); } \mathrm{MS}^{3}[285]: 241(95), \\
217(60), 199(60), 175(60), 151(20)\end{array}$ & Luteolin-7- $\alpha$ - $O$-glucuronide \\
\hline & & & 447 & $\begin{array}{l}\mathrm{MS}^{2}[447]: 285 ; \mathrm{MS}^{3}[285]: 243(50), \\
241(100), 199(60), 175(50), 151(15)\end{array}$ & Luteolin-7-O-glucoside \\
\hline 7 & 10.9 & 245,338 & 461 & $\begin{array}{l}\operatorname{MS}^{2}[461]: 446(1), 341(4), 323(3), \\
\text { 299(100); } S^{3}[299]: 284(100) ; \\
\text { MS }^{4}[284]: 256(40), 151(5) ; \mathrm{MS}^{5}[256]: \\
\text { 239(4), 227(100), 211(20), 200(10), } \\
\text { 122(60), 94(2) }\end{array}$ & Chrysoeriol-7- $\beta$ - $O$-glucoside \\
\hline 8 & 11.3 & 267,332 & 445 & $\begin{array}{l}\operatorname{MS}^{2}[445]: 269(100) \\
\text { 175(5);:MS } \\
3\end{array}$ & Apigenin-7- $\beta$ - $O$-glucuronide \\
\hline 9 & 11.5 & 290,328 & 359 & $\begin{array}{l}\operatorname{MS}^{2}[359]: 223(15), 197(25), 179(30), \\
\text { 161(100), 133(4); } \operatorname{MS}^{3}[179]: 161(25), \\
\text { 151(<1), 135(100) }\end{array}$ & Rosmarinic acid \\
\hline 10 & 12.5 & 290,323 & 537 & $\begin{array}{l}\mathrm{MS}^{2}[537]: 493 ; \mathrm{MS}^{3}[493]: 359(100), \\
357(15), 313(10), 295(3), 269(<1), \\
\text { 247(<1), 179(1), 161(1); } \mathrm{MS}^{4}[359]: \\
\text { 249(5), 223(10), 197(15), 179(25), } \\
\text { 161(100), 135(5) }\end{array}$ & $3^{\prime}-O$-(8"'-Z-Caffeoyl)rosmarinic acid \\
\hline
\end{tabular}


507 eluted in fractions $\mathbf{4}, \mathbf{6}, \mathbf{7 , 8}$ and 9 (in DMSO- $\mathrm{d}_{6}$ ).

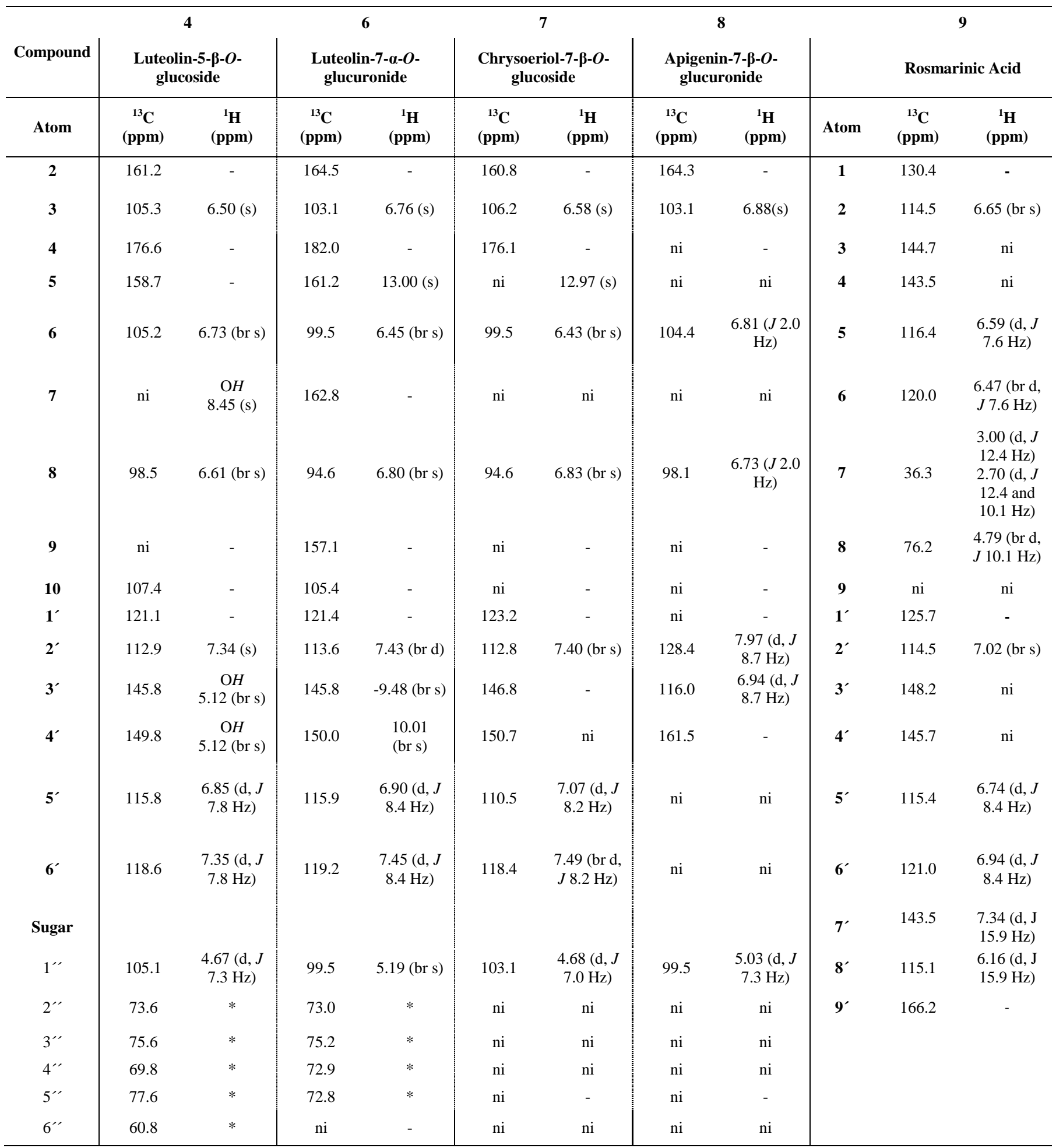


511 Table 3 - Linearity, LOD, LOQ and Intrumental and Method Precisions

Instrumental Precision Study

\begin{tabular}{|c|c|c|c|c|c|c|c|}
\hline $\begin{array}{l}\text { Standard } \\
\text { Compound }\end{array}$ & $\begin{array}{c}\text { Range concentration } \\
\qquad(\mu \mathrm{g} / \mathrm{mL})\end{array}$ & $\mathbf{n}^{\mathbf{a}}$ & $\begin{array}{l}\text { Slope }^{b}(\text { area } \\
\text { counts } / \mathbf{m g})\end{array}$ & $\begin{array}{c}\text { Intercept }^{\mathbf{b}} \\
\text { (area counts } \\
\text { /mg) }\end{array}$ & $\mathbf{R}^{2}$ & $\begin{array}{c}\text { LOD } \\
(\mu \mathrm{g} / \mathrm{mL})\end{array}$ & $\begin{array}{c}\text { LOQ } \\
(\mu \mathrm{g} / \mathrm{mL})\end{array}$ \\
\hline E-7O-G & $10.0-135.9$ & 5 & $144( \pm 1) \cdot 10^{6}$ & $-43( \pm 9) \cdot 10^{4}$ & 0.9999 & 2.0 & 6.2 \\
\hline N-7O-G & $5.0-67.9$ & 5 & $1797( \pm 6) \cdot 10^{5}$ & $-19( \pm 2) \cdot 10^{4}$ & 0.9999 & 1.0 & 3.0 \\
\hline L-7O-G & $45.3-300.0$ & 5 & $663( \pm 7) \cdot 10^{5}$ & $-4( \pm 1) \cdot 10^{5}$ & 0.9984 & 12.4 & 37.7 \\
\hline A-7O-G & $2.5-160.0$ & 5 & $848( \pm 8) \cdot 10^{5}$ & $-1( \pm 6) .10^{4}$ & 0.9988 & 7.3 & 22.1 \\
\hline RA & $14.9-120.0$ & 5 & $1343( \pm 9) \cdot 10^{5}$ & $-27( \pm 6) .10^{4}$ & 0.9995 & 3.3 & 9.9 \\
\hline
\end{tabular}

Instrumental Precision Study(intra- and inter-days variability of standard solutions injections)

\begin{tabular}{|c|c|c|c|c|c|}
\hline \multirow{3}{*}{$\begin{array}{c}\text { Standard } \\
\text { Compound }\end{array}$} & \multirow{3}{*}{$\begin{array}{c}\text { Concentration } \\
(\mu \mathrm{g} / \mathrm{mL})\end{array}$} & \multicolumn{4}{|c|}{$\%$ RSD } \\
\hline & & \multicolumn{2}{|c|}{ Repeatability $(\mathrm{n}=5)$} & \multicolumn{2}{|c|}{ Immediate precision $(\mathrm{n}=9)$} \\
\hline & & Retention Time (min) & Peak area & Retention Time (min) & Peak area \\
\hline \multirow{3}{*}{ E-7O-G } & 10 & 1.1 & 1.4 & 4.8 & 2.1 \\
\hline & 30 & 1.3 & 0.9 & 3.1 & 2.1 \\
\hline & 80 & 2.5 & 1.5 & 3.6 & 1.7 \\
\hline \multirow{3}{*}{$\mathrm{N}-7 \mathrm{O}-\mathrm{G}$} & 5 & 2.3 & 2.1 & 4.3 & 2.6 \\
\hline & 40 & 3.3 & 0.7 & 3.1 & 1.8 \\
\hline & 50 & 1.3 & 0.5 & 3.1 & 1 \\
\hline \multirow{3}{*}{$\mathrm{L}-7 \mathrm{O}-\mathrm{G}$} & 45 & 0.6 & 1.0 & 4.4 & 0.9 \\
\hline & 100 & 1.3 & 0.5 & 3.4 & 1.2 \\
\hline & 300 & 3.5 & 0.2 & 3.7 & 1.6 \\
\hline \multirow{3}{*}{ A-7O-G } & 10 & 0.2 & 1.6 & 3.4 & 2.5 \\
\hline & 80 & 3.4 & 1.1 & 4.3 & 1.6 \\
\hline & 160 & 0.9 & 1.1 & 3.9 & 2 \\
\hline \multirow{3}{*}{ RA } & 15 & 2.5 & 0.7 & 3.5 & 3.2 \\
\hline & 30 & 2.1 & 0.7 & 3 & 0.8 \\
\hline & 150 & 1.2 & 0.9 & 3.1 & 1.3 \\
\hline
\end{tabular}

Method Precision Study (intra- and inter-days variability of extractions obtained from a Thymus sample)

\begin{tabular}{|c|c|c|c|c|c|c|}
\hline \multicolumn{2}{|c|}{ Identified compound } & \multirow{2}{*}{$\begin{array}{c}\text { Standard } \\
\text { Compound (used } \\
\text { to quantify) }\end{array}$} & \multirow{2}{*}{$\begin{array}{c}\text { Mean } \\
\text { content }^{\mathrm{c}} \\
(\mathrm{mg} / \mathrm{g} \\
\text { extract) }\end{array}$} & \multicolumn{3}{|c|}{ \% RSD for compounds concentrations } \\
\hline $\begin{array}{c}\text { Fraction } \\
\mathbf{n}^{\mathbf{0}}\end{array}$ & Compound Name & & & $\begin{array}{c}\text { Repeatability } \\
\quad(n=5)\end{array}$ & $\begin{array}{c}\text { Immediate } \\
\text { Precision }(n=9)\end{array}$ & $\begin{array}{c}\text { Intermediate } \\
\text { Precision }(n=18)\end{array}$ \\
\hline 1 & Eriodictyol-di- $O$-hexoside & E-7O-G & $0.71 \pm 0.07$ & 5 & 7 & 11 \\
\hline 2 & Eriodictyol- $O$-hexoside & E-7O-G & $1.3 \pm 0.4$ & 10 & 12 & 33 \\
\hline 3 & Eriodictyol- $O$-hexoside & E-7O-G & $3.7 \pm 0.5$ & 4 & 7 & 12 \\
\hline 4 & Luteolin-5- $\beta$ - $O$-glucoside & $\mathrm{L}-7 \mathrm{O}-\mathrm{G}$ & $3.2 \pm 0.5$ & 2 & 4 & 16 \\
\hline 5 & Naringenin- $O$-hexoside & $\mathrm{N}-7 \mathrm{O}-\mathrm{G}$ & $1.8 \pm 0.2$ & 6 & 6 & 9 \\
\hline 6 & $\begin{array}{l}\text { Luteolin-7- } \alpha-O- \\
\text { glucuronide }\end{array}$ & $\mathrm{L}-7 \mathrm{O}-\mathrm{G}$ & $12 \pm 2$ & 4 & 5 & 20 \\
\hline 8 & $\begin{array}{l}\text { Apigenin-7- } \beta-O \text { - } \\
\text { glucuronide }\end{array}$ & A-7O-G & $9 \pm 2$ & 6 & 7 & 20 \\
\hline
\end{tabular}




\begin{tabular}{cccccc}
\hline 9 & Rosmarinic acid & RA & $10.4 \pm 0.6$ & 6 & 5 \\
\hline 10 & $\begin{array}{c}3^{\prime}-O-\left(8^{\prime \prime}-Z-\right. \\
\text { Caffeoyl)rosmarinic acid }\end{array}$ & RA & $2.3 \pm 0.9$ & 4 & 4 \\
\hline Total & --- & --- & $44 \pm 4$ & --- & ---
\end{tabular}

513 E-7O-G, eriodictyol-7- $O$-glucoside; N-7O-G, naringenin-7- $O$-glucoside; L-7O-G, luteolin-7- $O$-glucoside;

514 A-7O-G, apigenin-7- $O$-glucoside; RA, rosmarinic acid

515

$516{ }^{a}$ Number of points used for the regression of standard solutions. Injections were done in triplicate.

$517{ }^{b}$ The standard deviation in the slope and intercept of the regression line is shown in parentheses

$518{ }^{\mathrm{c}}$ Mean values \pm standard deviations 
521 Figure 1

522

523

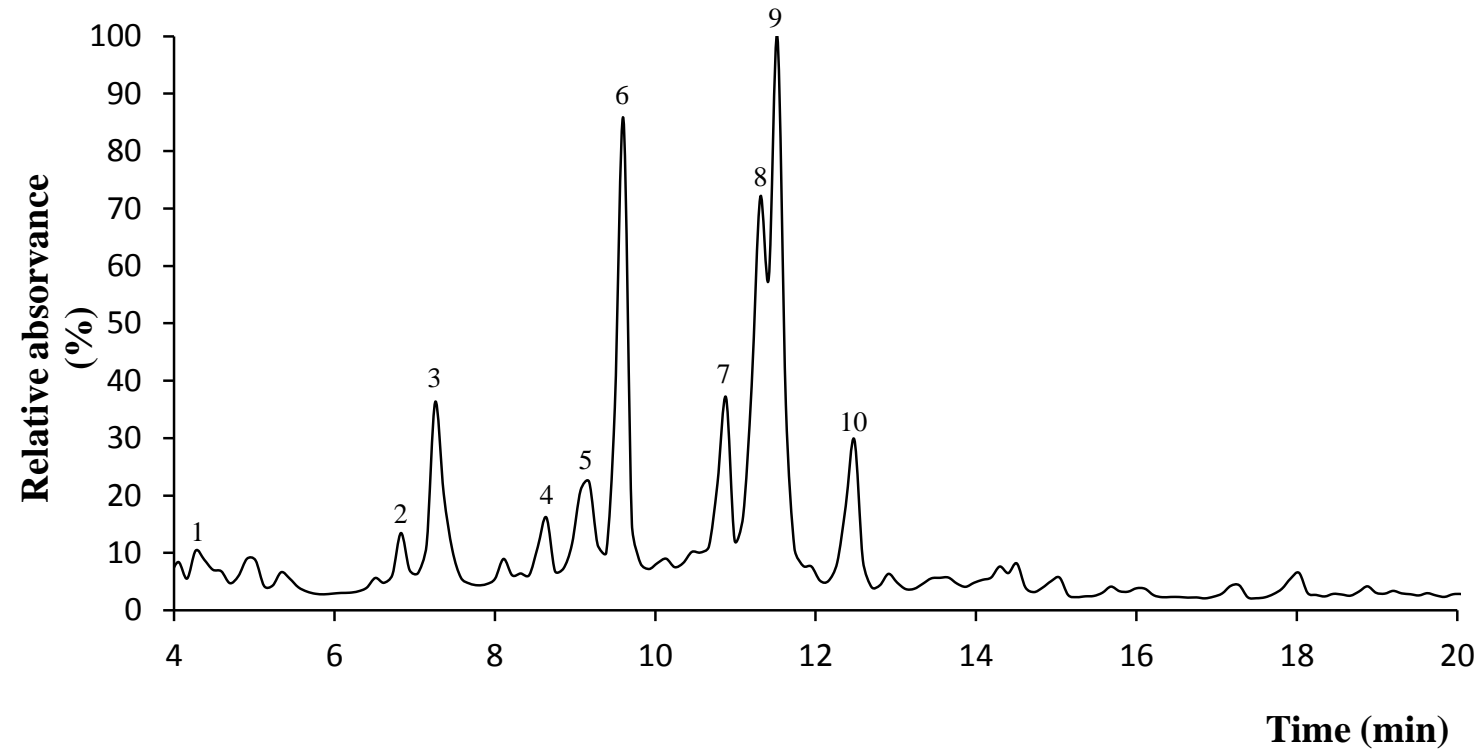

524

525 


\subsection{IDENTIFICATION OF PHENOLIC CONSTITUENTS OF CYTISUS}

\section{MULTIFLORUS}

Cytisus multiflorus is a Fabaceae plant known because of its potential health effects, including anti-inflammatory, antidiabetic and diuretic. However this specie has been far less studied than others of the same genus and, to the author's knowledge, its phenolic profile remains unknown. In this context, the present study aimed to characterize the phenolic composition of the $C$. multiflorus ethanolic extract.

The purified ethanolic extract of $C$. multiflorus was mainly rich in the flavone chrysin-7$O$ - $\beta$-D-glycopyranoside $(49.4 \pm 7.3 \mathrm{mg} / \mathrm{g}$ extract). Besides this compound, the extract also contained considerable amounts of a dihydroxyflavone isomer of chrysin $(21.8 \pm 3.8$ $\mathrm{mg} / \mathrm{g}$ extract), rutin (14.1 $1.7 \mathrm{mg} / \mathrm{g}$ extract), 2"-O-pentosyl-6-C-hexosyl-luteolin, 2"-Opentosyl-8- $C$-hexosyl-luteolin and 6"-O-(3-hydroxy-3-methylglutaroyl)-2"-O-pentosyl- $C$ hexosyl-apigenin, which are not commonly found in the Fabaceae family. Other flavones, including the common chrysin, orientin, luteolin-5-O-glucoside, luteolin-7-Oglucoside, apigenin and apigenin-7-O-glucoside, appeared as minor components. Moreover, it was possible to identify the following novel compounds in Cytisus: 2"-Opentosyl-6-C-hexosyl-apigenin, 2"-O-pentosyl-8-C-hexosyl-apigenin and 6"-O-(3hydroxy-3-methylglutaroyl)-2"-O-pentosyl-C-hexosyl-luteolin. Overall, the present work is a valuable contribution for the phenolic elucidation of Cytisus genus and of Fabaceae family.

The results obtained in this section of the Doctoral Thesis have been used to write the manuscript entitled "Identification of phenolic constituents of Cytisus multiflorus" which is published in Food Chemistry. 


\title{
Identification of phenolic constituents of Cytisus multiflorus
}

\author{
Olívia R. Pereira ${ }^{\mathrm{a}, \mathrm{b}}$, Artur M.S. Silva ${ }^{\mathrm{c}}$, Maria R.M. Domingues ${ }^{\mathrm{c}}$, Susana M. Cardoso ${ }^{\mathrm{a}, \mathrm{d}, *}$ \\ ${ }^{a}$ CERNAS - Escola Superior Agrária, Instituto Politécnico de Coimbra, Bencanta, 3040-316 Coimbra, Portugal \\ ${ }^{\mathrm{b}}$ Departamento de Tecnologias de Diagnóstico e Terapêutica, Escola Superior de Saúde, Instituto Politécnico de Bragança, Av. D. Afonso V, 5300-121 Bragança, Portugal

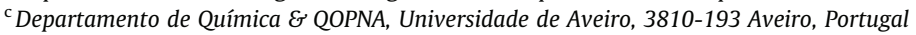 \\ d CIMO - Escola Superior Agrária, Instituto Politécnico de Bragança, Campus de Santa Apolónia, Apartado 1038, 5301 -854 Bragança, Portugal
}

\section{A R T I C L E I N F O}

\section{Article history:}

Received 28 March 2011

Received in revised form 11 May 2011

Accepted 14 September 2011

Available online 19 September 2011

\section{Keywords:}

Flavones

Chrysin-7-O- $\beta$-D-glucopyranoside

Apigenin derivatives

Luteolin derivatives

Electrospray ionisation-mass spectrometry NMR

\begin{abstract}
A B S T R A C T
The phenolic composition of the ethanolic extract obtained from the flowers of the medicinal plant Cytisus multiflorus has been elucidated by high performance liquid chromatography, electrospray mass spectrometry and nuclear magnetic resonance analysis. The extract was mainly composed of flavones, including the common chrysin, orientin, luteolin-5-O-glucoside, luteolin-7-O-glucoside, apigenin and apigenin-7-O-glucoside, which appeared as minor components. The major flavone in the extract was chrysin-7-O- $\beta$-D-glucopyranoside, and it also contained moderate amounts of a dihydroxyflavone isomer of chrysin, as well as of $2^{\prime \prime}-O$-pentosyl-6-C-hexosyl-luteolin, $2^{\prime \prime}-O$-pentosyl-8-C-hexosyl-luteolin and $6^{\prime \prime}-$ $O$-(3-hydroxy-3-methylglutaroyl)-2"-O-pentosyl-C-hexosyl-apigenin, which are not commonly found in the Fabaceae family. Other novel phenolic compounds found in the ethanolic extract of $C$. multiflorus comprised the flavones $2^{\prime \prime}-O$-pentosyl-6-C-hexosyl-apigenin, $2^{\prime \prime}-O$-pentosyl-8-C-hexosyl-apigenin and 6 " -0 -(3-hydroxy-3-methylglutaroyl)-2" -O-pentosyl-C-hexosyl-luteolin. The assessment of the biological activities of the main compounds of this extract are now keen, in order to determine their relevance in the beneficial properties of the plant.
\end{abstract}

(c) 2011 Elsevier Ltd. All rights reserved.

\section{Introduction}

Cytisus Desf. (Leguminosae-Cytiseae) is a large and diversified genus including approximately 60 species, which are particularly abundant around the Mediterranean Sea, although they are found in distinct geographic regions such as the north and south of Africa, the western and central Europe, the Black Sea and Turkey to the East (Cristofolini \& Conte, 2002; Cristofolini \& Troia, 2006). Plants of this genus exhibit bioactive properties, including antioxidant (Raja et al., 2007; Sundararajan et al., 2006), diuretic, hypnotic, anxiolytic (Nirmal, Babu, Harisudhan, \& Ramanathan, 2008; Siegel, 1976), antiparasitic (Di Giorgio et al., 2008) and antidiabetic (Castro, 1998, 2001) activities. The therapeutic properties and, in particular, the antioxidant activity of Cytisus is related to their high concentration of phenolic compounds (Luis, Domingues, Gil, \& Duarte, 2009). In general, plants of this genus are rich in flavonoids.

Abbreviations: CID, collision-induced dissociation; DAD, diode array; ESI-MS, electrospray ionisation-mass spectrometry; $\mathrm{MS}^{\mathrm{n}}$, Tandem mass spectrometry; GAE, gallic acid equivalent; HPLC, high-performance liquid chromatography; NMR, nuclear magnetic resonance.

* Corresponding author at: CERNAS - Escola Superior Agrária, Instituto Politécnico de Coimbra, Bencanta, 3040-316 Coimbra, Portugal. Tel.: +351 239 802940; fax: +351273239802979.

E-mail addresses: oliviapereira@ipb.pt (O.R. Pereira), scardoso@esac.pt (S.M. Cardoso).
Namely, Cytisus scoparius has been described to contain the flavone 6 "-O-acetyl-scoparin, the flavonols kaempferol, rutin, quercetin, quercitrin and isorhamnetin, and the isoflavones genistein and sarothamnoside, while the species Cytisus nigrians and Cytisus albus were shown to contain the isoflavones ononin and genistin (Hanganu, Vlase, \& Olah, 2010a, 2010b; Raja et al., 2007).

Cytisus multiflorus (L'Hér.) Sweet, also known as White Spanish Broom, is a leguminous shrub native from Iberian Peninsula that is distributed in the south-west Mediterranean region (Cristofolini \& Troia, 2006). This specie grows in poor and acidic soils, and frequently appears in degraded or marginal areas. It has a great number of white flowers with a valvular type pollen presentation system. The $C$. multiflorus is vastly used as an ornamental plant, as well as for animal nutrition. Other applications of this plant include the collection of their pollen for apiculture purposes and land fertilising in agriculture (Ciudad et al., 2004; Rodriguez-Riano, Ortega-Olivencia, \& Devesa, 1999, 2004; Rodriguez-Riano, Valtuena, \& Ortega-Olivencia, 2006).

C. multiflorus has also been used as an ethnopharmacological agent for centuries mainly due to its diuretic, anti-inflammatory, anti-hypertensor and antidiabetic properties (Gião et al., 2007). However, this specie has been far less studied than other of the same genus and, to our knowledge, its phenolic profile remains unknown. In this context, the present study intends to characterise the phenolic constituents of C. multiflorus, by high performance 
liquid chromatography associated with diode array detection (HPLC-DAD), electrospray mass spectrometry (ESI-MS and $\mathrm{MS}^{\mathrm{n}}$ ) and nuclear magnetic resonance analysis (NMR) techniques.

\section{Material and methods}

\subsection{Chemicals}

The phenolic standard gallic acid was obtained from Sigma Chemical Co. (St. Louis, MO, USA). Luteolin-8-C-glucoside (orientin), luteolin-7-O-glucoside, apigenin-7-O-glucoside, rutin and chrysin were obtained from Extrasynthese (Genay Cedex, France). Folin-Ciocalteu reagent, $\mathrm{Na}_{2} \mathrm{CO}_{3}$, formic acid and ethanol were purchased from Panreac (Barcelona, Spain). $n$-Hexane, methanol and acetonitrile with HPLC purity were purchased from Lab-Scan (Lisbon, Portugal). DMSO- $\mathrm{d}_{6}$ containing $0.03 \%$ of TMS was obtained from CortecNet (Paris, France). Water was treated in a Milli-Q water purification system (TGI Pure Water Systems, USA).

\subsection{Plant material}

The dried flowers of $C$. multiflorus were purchased from ERVITAL (Castro de Aire, Portugal). The plants have been cultivated under an organic regime and the flowers were collected in the Spring of 2009. After collection, these were dried at $25-30{ }^{\circ} \mathrm{C}$ in a ventilated incubator for approximately 5 days.

\subsection{Extraction of phenolic compounds}

The flowers of $C$. multiflorus ( $5 \mathrm{~g}$ ) were grounded and defatted with $150 \mathrm{ml} n$-hexane, for three times. The residue was extracted with $150 \mathrm{ml}$ of an $80 \%$ ethanol solution (v/v) at room temperature, for $1 \mathrm{~h}$ and the resulting mixture was filtered. The residue was extracted in the same conditions for three more times and the filtrated solutions were combined, concentrated, frozen at $-20^{\circ} \mathrm{C}$ and freeze-dried. The dried extract (ethanolic extract) of $C$. multiflorus was stored in vacuum, at a desiccator in dark, for subsequent use. This procedure was performed in triplicate.

\subsection{Purification of phenolic compounds}

The ethanolic extract of $C$. multiflorus was purified in order to obtain a suitable sample for NMR analyses. For that, $55 \mathrm{mg}$ of this extract were dissolved in $3 \mathrm{ml}$ of water and eluted in a Strata SPE C18-E cartridge Sephadex (2 g, Waters, Milford, MA, USA). The cartridge was then washed with $5 \mathrm{ml}$ of water, for three times, and the phenolic compounds were recovered by elution with $10 \mathrm{ml}$ of methanol. Following crystallization by evaporation of the solvent to a minimum volume (approximately $1 \mathrm{ml}$ ), the supernatant was removed by decantation and the precipitated material was solubilised in DMSO- $\mathrm{d}_{6}$ for NMR analysis.

\subsection{Quantification of total phenolic compounds}

The total concentration of phenolic compounds in the ethanolic extract of $C$. multiflorus was determined according to the adapted Folin-Ciocalteu colorimetric method (Singleton \& Rossi, 1965) described by Ferreira et al. (2002). The results of the total phenolic compounds were expressed as gallic acid equivalent (mg GAE)/g dried weight of plant material using a calibration curve of gallic acid as standard $(5-37.5 \mu \mathrm{g} / \mathrm{ml})$. All samples were tested in triplicate.

\subsection{HPLC apparatus and chromatographic conditions}

The HPLC analysis was performed on a Varian 9010 separation module equipped with PDA Varian Prostar detector. The data acquisition and remote control of the HPLC system were conducted by Varian Star chromatography Workstation ${ }^{\circledR}$ (Lake Forest, CA, USA) software. The column used was a $250 \mathrm{~mm} \times 4 \mathrm{~mm}$ id, $5 \mu \mathrm{m}$ bead diameter, end-capped Nucleosil C18 (Macherey-Nagel), and its temperature was maintained at $30^{\circ} \mathrm{C}$.

The flow rate used was $1 \mathrm{ml} / \mathrm{min}$ and the gradient elution was carried out with a mixture of two solvents. Solvent A consisted of $0.1 \%(\mathrm{v} / \mathrm{v})$ of formic acid in water and solvent B consisted of acetonitrile, which were degassed and filtrated before use. The solvent gradient consisted in a series of linear gradients, starting from $10 \%$ to $30 \%$ of solvent B over $20 \mathrm{~min}$, from $30 \%$ to $100 \%$ of solvent B over $5 \mathrm{~min}$, decreasing to $10 \%$ of solvent B after $5 \mathrm{~min}$ followed by the return to the initial conditions. For the HPLC analysis, the samples $(10 \mathrm{mg}$ ) were dissolved in $1 \mathrm{ml}$ of methanol, filtered through a $0.2 \mu \mathrm{m}$ Nylon membrane (Whatman) and $10 \mu \mathrm{l}$ of each solution was injected. The UV-Vis spectra were recorded between 220 and $500 \mathrm{~nm}$ and the chromatographic profiles were recorded at $280 \mathrm{~nm}$.

\subsection{Identification of the phenolic compounds}

Compounds for which standards were available were first identified by comparison of the retention times and UV-Vis spectra of the corresponding HPLC peaks. Further analysis by electrospray ionisation mass spectrometry (ESI-MS and ESI-MS ${ }^{\mathrm{n}}$ ) allowed the confirmation of their structure (in the case of previous identification by HPLC-DAD) or to obtain structural information on the eluting compounds. In order to have enough amount of sample to carry out this latter analysis, the peak-forming fractions from three independent runs were collected manually according to the visualisation of the UV profile and were freeze-dried. Note that as the NMR analysis requires an amount of sample of approximately $5 \mathrm{mg}$, this technique was not performed for the HPLC collected fractions (the collection procedure only resulted in micrograms quantities of sample). Still, NMR assays were performed on a purified fraction of the ethanolic extract, in order to elucidate the structure of the main compound in the extract.

\subsection{Quantification of the identified phenolic compounds}

Fraction 1 (2"-O-pentosyl-6-C-hexosyl-luteolin), fraction 2 (2"-O-pentosyl-8-C-hexosyl-luteolin) and fraction 3 (orientin), were quantified using orientin as the reference compound as, in accordance to their UV-Vis and MS spectra, they were mainly rich in luteolin-glucoside derivatives. In a similar approach, fraction 4 (2"-O-pentosyl-8-C-hexosyl-apigenin), fraction 5 (2"-O-pentosyl6-C-hexosyl-apigenin), fraction 7 [6"-O-(3-hydroxy-3-methylglutaroyl)-2"-O-pentosyl-C-hexosyl-apigenin, quercetin-3-O-glucoside and luteolin-7-O-glucoside], fraction 8 (apigenin-7-O-glucoside) and fraction 11 (apigenin) were quantified using apigenin-7-O-glucoside as reference. Moreover, fraction 6 [rutin, luteolin-5-O-glucoside and 6"-O-(3-hydroxy-3-methylglutaroyl)-2"-O-pentosyl-Chexosyl-luteolin] was quantified using rutin as reference, while chrysin was used as the reference for the quantification of phenolic compounds in fractions 9 (chrysin-7-O-glucoside), 10 (dihydroxyflavone chrysin isomer) and 12 (chrysin). Five-points calibration curves were used for each standard. In particular, for orientin, the tested range was $0.013-0.1 \mathrm{mg} / \mathrm{ml}$ and the achieved equation was $y=6 \mathrm{E}+07 x-286,681$, with $R^{2}$ value of $0.9995(n=13)$. The quantification limit (LQ) and detection limit (LD) of this compound were 0.0175 and $0.0058 \mathrm{mg} / \mathrm{ml}$, respectively. For apigenin-7-O-glucoside, the tested range was $0.003-0.04 \mathrm{mg} / \mathrm{ml}$, the equation was $y=8 \mathrm{E}+07 x-52,202$ with $R^{2}$ value of $0.9996(n=13)$. LQ and LD were 0.0131 and $0.0043 \mathrm{mg} / \mathrm{ml}$, respectively. The calibration curves of the phenolic standards rutin and chrysin were performed for ranges of $0.018-0.14$ and $0.006-0.374 \mathrm{mg} / \mathrm{ml}$, respectively. The 
respective equations were $Y=4 \mathrm{E}+07 x-401,004 \quad(n=13)$ and $Y=1 \mathrm{E}+08 x-354,600(n=13)$, with LQ values of 0.0625 and 0.0160 $\mathrm{mg} / \mathrm{ml}$ (respectively) and LD values of 0.018 and $0.0053 \mathrm{mg} / \mathrm{ml}$, respectively.

\subsection{Mass spectrometry analysis by ESI-MS and ESI-MS ${ }^{n}$}

The HPLC fractions or the phenolic standards were dissolved in methanol and directly injected into the ESI source by means of a syringe pump, at a flow rate of $8 \mu \mathrm{l} / \mathrm{min}$. ESI-MS analyses were performed in the negative ion mode within the $m / z$ range $50-1000$, using a Linear Ion trap LXQ instrument (ThermoFinnigan, San Jose, CA, USA) equipped with Xcalibur ${ }^{\circledR}$ software (ThermoFinnigan, San Jose, CA, USA). Typical ESI conditions were: nitrogen sheath gas 30 psi, spray voltage $4.7 \mathrm{kV}$, capillary temperature $275^{\circ} \mathrm{C}$, capillary voltage $-37.0 \mathrm{~V}$ and tube lens voltage $-81.89 \mathrm{~V}$. CID-MS/MS and $\mathrm{MS}^{\mathrm{n}}$ experiments were performed on mass-selected precursor ions using a standard isolation and excitation configuration. Full scan data acquisition was performed from $\mathrm{m} / \mathrm{z} 100$ to $\mathrm{m} / \mathrm{z} 1000$ in MS scan mode.

\subsection{Nuclear magnetic resonance (NMR) studies}

${ }^{1} \mathrm{H}$ and ${ }^{13} \mathrm{C}$ NMR spectra of the purified phenolic extract were recorded at $298 \mathrm{~K}$ on a Bruker Avance 500 spectrometer operating at 500.13 and $125.77 \mathrm{MHz}$, respectively. The phase sensitive ${ }^{1} \mathrm{H}$-detected $\left({ }^{1} \mathrm{H},{ }^{13} \mathrm{C}\right) \mathrm{gHSQC}$ (heteronuclear single quantum coherence, using gradient pulses for selection) spectrum was recorded with 216 transients over 256 increments (zero-filled to 512) and $2 \mathrm{~K}$ data points with spectral widths of $4500 \mathrm{~Hz}$ in $\mathrm{F}_{2}$ and $20 \mathrm{kHz}$ in $\mathrm{F}_{1}$. The repetition time was $1.9 \mathrm{~s}$. A cosine multiplication was applied in both dimensions. The delays were adjusted according to a coupling constant ${ }^{1} J(\mathrm{CH})$ of $147 \mathrm{~Hz}$. The gHMBC (heteronuclear multiple quantum coherence, using gradient pulses for selection) spectrum was recorded with 240 transients over 256 increments (zero-filled to $1 \mathrm{~K}$ ) and $2 \mathrm{~K}$ data points with spectral widths of $4500 \mathrm{~Hz}$ in $\mathrm{F}_{2}$ and $25 \mathrm{kHz}$ in $\mathrm{F}_{1}$. The repetition time was $1.9 \mathrm{~s}$. A sine multiplication was applied in both dimensions. The low-pass $J$-filter of the experiment was adjusted for an average coupling constant ${ }^{1} \mathrm{~J}(\mathrm{CH})$ of $147 \mathrm{~Hz}$ and the long-range delay utilised to excite the heteronuclear multiple quantum coherence was optimised for $7 \mathrm{~Hz}$. Chrysin (Sigma) was used as a reference compound for the structural elucidation of the purified ethanolic extract. According to the interpretation of its ${ }^{1} \mathrm{H},{ }^{13} \mathrm{C}$ NMR, HSQC, COSY and HMBC spectra the ${ }^{1} \mathrm{H}$ and ${ }^{13} \mathrm{C}$ NMR chemical shifts of chrysin were assigned as follow: ${ }^{1} \mathrm{H}$ NMR: $\delta=6.22$ (d, $J=2.1 \mathrm{~Hz}, \mathrm{H}-6), 6.53$ (d, $J=2.1 \mathrm{~Hz}, \mathrm{H}-8), 6.98$ (s, H-3), 7.54-7.64 (m, H-3', $\left.4^{\prime}, 5^{\prime}\right), 8.07$ (dd, $J=1.7$ and $7.9 \mathrm{~Hz}$, H-2', $\left.6^{\prime}\right) ;{ }^{13} \mathrm{C}$ NMR: $\delta=94.1$ (C-8), 99.0 (C-6), 104.0 (C-10), 105.2 (C-3), $126.4\left(\mathrm{C}-2^{\prime}, 6^{\prime}\right), 129.2\left(\mathrm{C}-3^{\prime}, 5^{\prime}\right), 130.7\left(\mathrm{C}-1^{\prime}\right), 132.1\left(\mathrm{C}-4^{\prime}\right)$, 157.5 (C-9, 161.5 (C-5), 163.2 (C-2), 164.4 (C-7), 181.9 (C-4).

\section{Results and discussion}

The ethanolic extract of C. multiflorus represented $32 \%$ of the dried plant mass and its total phenolic compounds accounted for $140 \pm 12 \mathrm{mg} \mathrm{GAE} / \mathrm{g}$ of extract (data not shown). This amount corresponds to a recovery of $44.7 \pm 4.0 \mathrm{mg}$ GAE/g dried plant and thus, it is higher than those values reported by Gião et al. (2007)) for extracts of $C$. multiflorus obtained by infusion or boiling $(12.9 \mathrm{mg} / \mathrm{g}$ or $26.2 \mathrm{mg} / \mathrm{g} \mathrm{GAE} / \mathrm{g}$ dried plant, respectively).

\subsection{Identification of the phenolic compounds of the ethanolic extract of C. multiflorus}

In order to characterise the phenolic compounds of the ethanolic extract of $C$. multiflorus, this was further analysed by
HPLC-DAD. The corresponding chromatogram, at $280 \mathrm{~nm}$ is shown in Fig. 1. Only four of the twelve fractions matched with the available phenolic standards, namely fractions $3,6,8$ and 12 , which corresponded to orientin, rutin, apigenin-7-O-glucoside and chrysin, respectively. These four assignments, as also the identification of the remaining phenolic components in the ethanolic extract of $C$. multiflorus were elucidated considering the HPLC-DAD figures, together with electrospray ionisation mass spectrometry (ESI-MS and $\mathrm{MS}^{\mathrm{n}}$ ) data. Moreover, the NMR analysis of the purified ethanolic extract provided crucial information for the assignment of the main phenolic compound in the extract (fraction 9).

Table 1 summarises the HPLC-DAD and MS data obtained for each of the analysed fractions. MS analysis was preferentially obtained in the negative mode, because of its higher sensitivity in the detection of the distinct classes of phenolic compounds (Cuyckens \& Claeys, 2004) although in some cases, analysis in the positive mode was also used in order to confirm the data from the negative mode (data not shown). Together with the NMR analysis, it is possible to conclude that the ethanolic extract of $C$. multiflorus is mainly rich in flavones. Indeed, besides this class of compounds, only two derivatives of quercetin (flavonol) were found. The following sections will focus on the assignments of the structural features of these compounds.

\subsubsection{Chrysin derivatives}

Besides chrysin, which appeared in the HPLC-DAD profile as a minor component in fraction 12 (eluted at $23.7 \mathrm{~min}$ ), the ethanolic extract of $C$. multiflorus contained two other chrysin derivatives, which were eluted in fractions 9 and 10 . The analysis of these fractions by ESI-MS/MS, together with the analysis of a purified fraction by NMR, allowed to fully elucidate the structure of the compound in fraction 9 and to obtain some structural features on the chrysin derivative detected in fraction 10.

The ESI-MS spectrum of fraction 9 showed two distinct molecular species (at $m / z 451$ and 461 ), which corresponded to the ionisation of the same compound. In fact, the ESI-MS/MS spectra of those two molecular ions showed similar product ions, namely at $\mathrm{m} / \mathrm{z} 415$ and 253 . The formation of the product ion at $\mathrm{m} / \mathrm{z} 415$ corresponded to the loss of $36 \mathrm{Da}$ (for the molecular specie at $m / z 451$ ) and $46 \mathrm{Da}$ (for the molecular specie at $\mathrm{m} / \mathrm{z} 461$ ), thus suggesting that they respectively correspond to the chloride adduct $[\mathrm{M}+\mathrm{Cl}]^{-}$and formic acid adduct $\left[\mathrm{M}+\mathrm{CH}_{2} \mathrm{O}_{2}\right]^{-}$of the compound (MW $416 \mathrm{Da}$ ). Moreover, the presence of the product ion at $\mathrm{m} / \mathrm{z}$ 253 in the ESI-MS/MS spectrum of the adducts (at $\mathrm{m} / z 451$ and $\mathrm{m} / \mathrm{z} 461$ ) suggested that the compound of MW 416 Da was a chrysin derivative. This hypothesis was supported by the $\mathrm{MS}^{3}$ spectrum of the ion at $m / z 253$ and also by the UV-Vis spectrum of fraction 9 , which were similar to that of the reference compound. Moreover, the main product ion of the $\mathrm{MS}^{3}$ spectrum of the $[\mathrm{M}+\mathrm{Cl}-\mathrm{HCl}]^{-}$ at $m / z 415$ is the ion at $m / z 253$, which was formed by the loss of $162 \mathrm{Da}$. These results suggested that the main compound in fraction 9 was an hexoside derivative of chrysin.

The total structural elucidation of the phenolic compound detected in fraction 9 (MW $416 \mathrm{Da}$ ) was accomplished by NMR analysis. The assays were conducted on a purified sample, in order to simplify the interpretation of the spectra. Still, it must be noted that NMR experiments were also performed on the ethanolic extract (non purified) in order to assure that its main compound corresponded to that of the purified fraction (data not shown). As can be concluded from Fig. 2 and Table 2, all the NMR signals corresponded to one compound, suggesting that the purified procedure was efficient. The ${ }^{1} \mathrm{H}$ and ${ }^{13} \mathrm{C}$ NMR chemical shifts presented in Table 2 were assigned according to the analysis of its ${ }^{1} \mathrm{H}$ (Fig. 2), ${ }^{13} \mathrm{C}$, COSY, HSQC, and HMBC NMR spectra (data not shown) and further comparison to those of chrysin and to the literature data (El Antri et al., 2004). All the signals in the spectra 


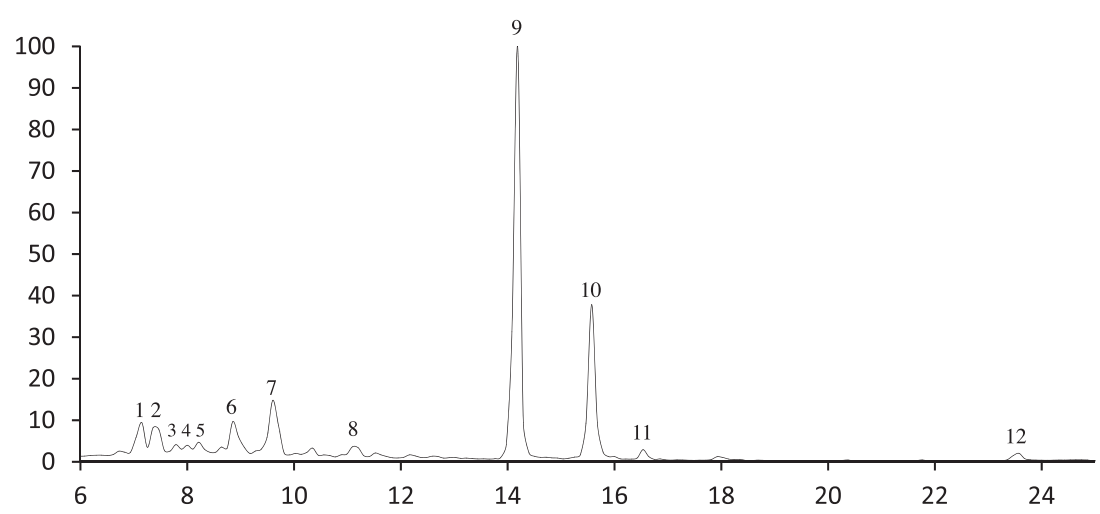

Fig. 1. Chromatographic profile of ethanolic fractions of Cytisus multiflorus at $280 \mathrm{~nm}$. The numbers on the figure correspond to the fractions that were collected for by ESI-MS analysis.

Table 1

Identification of HPLC eluting fractions by HPLC-DAD and ESI-MS from the ethanolic extract of Cytisus multiflorus.

\begin{tabular}{|c|c|c|c|c|c|}
\hline Peak & $\begin{array}{l}\mathrm{RT} \\
(\min )\end{array}$ & $\lambda_{\max }$ & $\begin{array}{l}\text { Compound } \\
\text { (MW) }\end{array}$ & Main fragment $\mathrm{ESI}^{-} \mathrm{MS}^{\mathrm{n}}$ & Compound \\
\hline 1 & 7.2 & $\begin{array}{l}256, \\
266 \\
347\end{array}$ & 580 & $\operatorname{MS}^{2}$ [579]: 459(50), 429(100), 357(20), 327(40), 309(5), 285(1) & $2^{\prime \prime}$-O-pentosyl-6-C-hexosyl-luteolin \\
\hline 2 & 7.5 & $\begin{array}{l}257, \\
266, \\
346\end{array}$ & 580 & $\begin{array}{l}\mathrm{MS}^{2}[579]: \text { 459(75), 449(15), 429(100), 357(65), 327(100), 309(5), 297(<1), 285(<1); } \\
\mathrm{MS}^{3}[459]: 327(100) ; \mathrm{MS}^{4}[327]: 299(100), 284(15), 255(2) ; \mathrm{MS}^{5}[299]: 271(15), \\
\text { 255(100), 240(10), 213(25), 199(3), 175(15), 165(5), 163(1) }\end{array}$ & $2^{\prime \prime}$-O-pentosyl-8-C-hexosyl-luteolin \\
\hline 3 & 7.9 & $\begin{array}{l}256, \\
266 \\
345\end{array}$ & 448 & $\mathrm{MS}^{2}$ [447]: 357(40), 327(100), 285(10); $\mathrm{MS}^{3}$ [357]: 339(35), 297(100), 285(90) & Orientin \\
\hline 4 & 8.1 & $\begin{array}{l}267, \\
338\end{array}$ & 564 & $\begin{array}{l}\mathrm{MS}^{2}[563]: 545(<1), 473(<1), 443(2), 413(100), 341(<1), 311(<1), 293(4) ; \mathrm{MS}^{3}[413]: \\
\text { 293(100); } \mathrm{MS}^{4}[293]: \text { 275(7), 265(30), 249(100), 175(60) }\end{array}$ & $2^{\prime \prime}$-O-pentosyl-8-C-hexosyl-apigenin \\
\hline \multirow[t]{2}{*}{5} & 8.3 & $\begin{array}{l}267, \\
338\end{array}$ & 564 & $\begin{array}{l}\operatorname{MS}^{2}[563]: ~ 443(4), 413(100), 293(8) ; \mathrm{MS}^{3} \text { [413]: 293; } \mathrm{MS}^{4} \text { [293]: 265(40), 249(100), } \\
\text { 175(50) }\end{array}$ & $2^{\prime \prime}$-O-pentosyl-6-C-hexosyl-apigenin \\
\hline & & & 356 & $\begin{array}{l}\mathrm{MS}^{2}[355]: \text { 337(15), 199(5), 183(20), 179(100), 175(15), 161(15), 149(2), 143(15), } \\
\text { 131(4), 113(10); } \mathrm{MS}^{3} \text { [179]: 161(45), 143(100), 119(3), 89(50) }\end{array}$ & Unknown \\
\hline \multirow[t]{3}{*}{6} & 9.3 & $\begin{array}{l}255, \\
352\end{array}$ & 610 & $\begin{array}{l}\mathrm{MS}^{2} \text { [609]: 343(7), 301(100); } \mathrm{MS}^{3} \text { [301]: 273(10), 257(10),) 179(100), 151(60); } \mathrm{MS}^{4} \\
\text { [179]: 151(100); } \mathrm{MS}^{5}[151]: 107\end{array}$ & Rutin \\
\hline & & & 448 & $\begin{array}{l}\text { MS }^{2} \text { [447]: 285(100); } \text { MS }^{3} \text { [285]: 257(7), 241(100), } 217 \text { (45), } 199 \text { (75), } 175 \text { (60), } 151 \\
\text { (12) }\end{array}$ & Luteolin-5-O-glucoside \\
\hline & & & 724 & $\begin{array}{l}\mathrm{MS}^{2} \text { [723]: 661(5), } 621(15), 579(100), 459(15), 357(15), 327(15) ; \mathrm{MS}^{3}[579]: 459(80), \\
\text { 429(90), } 357(70), 327(100) ; \mathrm{MS}^{4}[459]: 327(100) ; \mathrm{MS}^{5}[327]: 299(100), 284(20), \\
\text { 255(2); } \mathrm{MS}^{5} \text { [299]: } 255\end{array}$ & $\begin{array}{l}6^{\prime \prime}-O \text {-(3-hydroxy-3- } \\
\text { methylglutaroyl)-2"-O-pentosyl- } C \text { - } \\
\text { hexosyl-luteolin }\end{array}$ \\
\hline \multirow[t]{3}{*}{7} & 9.7 & $\begin{array}{l}266, \\
342\end{array}$ & 708 & $\begin{array}{l}\mathrm{MS}^{2} \text { [707]: 645(7), 605(10), 563(100); } \mathrm{MS}^{3}[563]: 443(5), 413(100), 293(10) ; \mathrm{MS}^{4}[413]: \\
293 ; \mathrm{MS}^{5} \text { [293]: 249(100), 205(1), 175(20) }\end{array}$ & $\begin{array}{l}6^{\prime \prime}-O-(3 \text {-hydroxy-3- } \\
\text { methylglutaroyl)-2"-O-pentosyl- } C \text { - } \\
\text { hexosyl-apigenin }\end{array}$ \\
\hline & & & 464 & $\begin{array}{l}\mathrm{MS}^{2} \text { [463]: 301(100), 300(20); } \mathrm{MS}^{3} \text { [301]: 283(3), 273(15), 257(15), 229(3), 193(5), } \\
\text { 179(100), 151(65), 107(2) }\end{array}$ & Quercetin-3-O-glucoside \\
\hline & & $\begin{array}{l}255, \\
262, \\
347\end{array}$ & 448 & $\begin{array}{l}\mathrm{MS}^{2}[447]: \text { 285; } \mathrm{MS}^{3}[285]: \text { 267(12), 257(20), 243(50), 241(100), 217(50), 201(15), } \\
\text { 199(65), 197(10), 175(60), 151(15) }\end{array}$ & Luteolin-7-O- glucoside \\
\hline 8 & 11.3 & $\begin{array}{l}266, \\
342\end{array}$ & 432 & $\operatorname{MS}^{2}[431]: 269$ & Apigenin-7-O- glucoside \\
\hline \multirow[t]{2}{*}{9} & 14.3 & $\begin{array}{l}267, \\
303\end{array}$ & 462 & $\operatorname{MS}^{2}$ [461]: 415 (15), 253(100); $\operatorname{MS}^{3}$ [253]: 209(100), 181(4), 153(1) & Chrysin-7-O-glucoside \\
\hline & & & 452 & $\operatorname{MS}^{2}[451]:$ 415(5), 253(100); $\mathrm{MS}^{3}$ [253]: 209(100), 181(4), 151(1) & \\
\hline 10 & 15.7 & $\begin{array}{l}267, \\
303\end{array}$ & 254 & $\begin{array}{l}\mathrm{MS}^{2}[253]: \text { 225(5), 209(100), 167(3), 165(5), 159(5), 151(4), 113(10), 107(3); } \mathrm{MS}^{3}[209] \text { : } \\
\text { 181(40), 167(5), 165(50), 153(15) }\end{array}$ & Chrysin isomer \\
\hline 11 & 16.7 & ND & 270 & $\begin{array}{l}\text { MS }^{2}[269]: \text { 251(40), 241(25), 227(15), 225(100), 207(20), 201(40), 197(20), 183(10), } \\
\text { 181(35), 175(30), 169(10), 151(3), 149(5) }\end{array}$ & Apigenin \\
\hline 12 & 23.7 & $\begin{array}{l}267, \\
313\end{array}$ & 254 & $\operatorname{MS}^{2}[253]: 209$ & Chrysin \\
\hline
\end{tabular}

corroborated the presence of chrysin-7-O- $\beta$-D-glucopyranoside in the extract. Indeed, besides the characteristic signals of the chrysin aglycone, the spectra showed typical ${ }^{1} \mathrm{H}$ and ${ }^{13} \mathrm{C}$ chemical shifts for $\beta-\operatorname{Glcp}\left(\delta_{\mathrm{H}-1}=5.1 \mathrm{ppm}, J=7.5 \mathrm{~Hz}, \delta_{\mathrm{C}-1}=100.6 \mathrm{ppm}\right)$. Moreover, the long-range $\mathrm{H}-1^{\prime \prime} \rightarrow \mathrm{C}-7$ correlation in the gHMBC spectrum, allowed to confirm that the anomeric carbon of glucose was linked to $\mathrm{C}-7$ of the flavone skeleton. Thus, overall, the mass spectrometry and NMR analysis allowed the conclusion that the compound eluted in fraction 9 corresponded to the known flavonoid chrysin-7-O- $\beta$-D-glucopyranoside. To the best of our knowledge, this flavone was detected for the first time in the Fabaceae family.

Concerning fraction 10, its ESI-MS spectrum showed the molecular ion at $\mathrm{m} / \mathrm{z} 253$ (negative mode) or at $\mathrm{m} / \mathrm{z} 255$ (positive mode), suggesting the presence of a chrysin isomer. This hypothesis was 


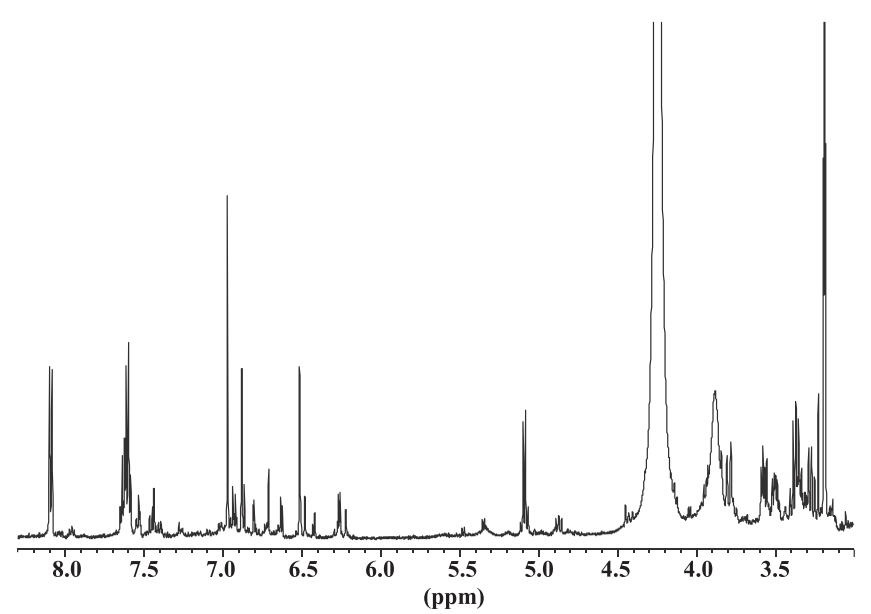

Fig. 2. ${ }^{1} \mathrm{H}$ NMR spectrum of a purified fraction from the ethanolic extract of Cytisus multiflorus.

further confirmed by the UV-Vis spectrum of that fraction, which was similar to that of fractions 9 and 12 . Attending to these data, we tentatively assigned the compound in fraction 10 to a dihydroxyflavone (MW $254 \mathrm{Da}$ ), although the position of the hydroxyl groups in ring A could not be determined.

\subsubsection{Luteolin derivatives}

Luteolin derivatives in C. multiflorus were mostly O-glycosyl-Cglycosyl-flavones, with the $\mathrm{O}$-glycosylation located on the sugar moiety of a C-hexosyl-flavone skeleton, as shown in Fig 3. Indeed, as observed in Table 1, the interpretation of the fragmentation pathway of the molecular ions in the HPLC-DAD fractions allowed the identification of three of these derivatives, namely in fractions 1,2 and 6 . In more detail, the analysis of the negative ESI-MS spectra of fractions 1 and 2 showed a $[\mathrm{M}-\mathrm{H}]^{-}$ion at $\mathrm{m} / \mathrm{z} 579$, and its $\mathrm{MS}^{2}$ spectrum revealed the ions at $m / z 459,429,357$, 327 , and 285 . This latter product ion, as also the UV-Vis spectra of these two fractions, supported the occurrence of luteolin derivatives. Moreover, the base peak at $m / z 429(-150 \mathrm{Da})$ was formed by the loss of a pentose sugar and is indicative of a $O$-pentosyl group in those compounds (Ferreres, Gil-Izquierdo, Andrade, Valentao, \& Tomas-Barberan, 2007), while the ion at $\mathrm{m} / \mathrm{z} 459$ $(-120 \mathrm{Da})$ corresponds to the intramolecular breakage of the hexose on the C-glycosyl-flavone unit (Cuyckens \& Claeys, 2004) and is characteristic of $2^{\prime \prime}$-substituted hexoses (Ferreres, Gil-Izquierdo, et al., 2007). The existence of $2^{\prime \prime}-O$-glycosyl-C-glycosyl-flavones in fractions 1 and 2 was also supported by the MS analysis in the positive mode (data not shown). Indeed, the analysis of the $[\mathrm{M}+\mathrm{H}]^{+}$ion at $\mathrm{m} / \mathrm{z} 581$ showed the product ions at $\mathrm{m} / \mathrm{z} 431,329$ and 287, that correspond to some of the most abundant ions in the negative ion analysis. Moreover, the fragmentation pathway of the base peak at $\mathrm{m} / \mathrm{z} 449$ corroborated the hypothesis of a luteolin-C-glucoside derivative (Ioset et al., 2007) and the loss of $132 \mathrm{Da}$ from the product ion at $\mathrm{m} / \mathrm{z} 449$ confirmed the presence of a pentose unit with a 0 -linkage (Cuyckens \& Claeys, 2004; Han et al., 2008; Regos, Urbanella, \& Treutter, 2009; Ye, Yan, \& Guo, 2005).

Overall, the above results allowed the detection, for the first time, of two isomers of $2^{\prime \prime}$-O-pentosyl-C-hexosyl-luteolin derivatives in Cytisus. The presence of these two isomers in contiguous fractions were confirmed by HPLC-MS analysis (results not shown). Moreover, considering that in nature the $C$-glycosyl moieties appear almost exclusively at 6 and/or 8-positions of flavones (Cuyckens \& Claeys, 2004) and that the 8-C-glycosyl-luteolin isomer elutes before the 6-C-glycosyl-luteolin under reversed phase conditions (Kazuno, Yanagida, Shindo, \& Murayama, 2005; Pereira, Yariwake, \& McCullagh, 2005; Piccinelli et al., 2008), the phenolic compounds in fractions 1 and 2 were respectively assigned to $2^{\prime \prime}-$ $O$-pentosyl-6-C-hexosyl-luteolin and 2"-O-pentosyl-8-C-hexosylluteolin. The structures of these two compounds are depicted in Fig. 3.

Table 2

Chemical shifts $\left(\delta\right.$ ) of chrysin-7-O- $\beta$-D-glucopyranoside obtained from purified ethanolic extract of Cytisus multiflorus (in DMSO- $\mathrm{d}_{6}$ ).<smiles>C[C@@H]1[C@@H](CO)O[C@H](Oc2cc(O)c3c(=O)cc(-c4ccccc4)oc3c2)[C@H](O)[C@H]1O</smiles>

\begin{tabular}{|c|c|c|c|c|c|}
\hline Atom & ${ }^{13} \mathrm{C}$ & ${ }^{1} \mathrm{H}$ & Atom & ${ }^{13} \mathrm{C}$ & ${ }^{1} \mathrm{H}$ \\
\hline 2 & 164.6 & - & Glucose & & \\
\hline 3 & 106.0 & 6.97 & $1^{\prime \prime}$ & 100.6 & $5.10(J=7.5 \mathrm{~Hz})$ \\
\hline 4 & 182.8 & - & $2^{\prime \prime}$ & 73.7 & $3.34(\mathrm{~m})$ \\
\hline 5 & 161.7 & - & $3^{\prime \prime}$ & 76.9 & $3.37(\mathrm{dd}, J=9.6,7.9 \mathrm{~Hz})$ \\
\hline 6 & 100.3 & $6.89(\mathrm{~d}, J=2.2 \mathrm{~Hz})$ & $4^{\prime \prime}$ & 70.1 & $3.28(\mathrm{t}, J=9.6 \mathrm{~Hz})$ \\
\hline 7 & 163.9 & - & $5^{\prime \prime}$ & 77.7 & $3.50(\mathrm{~m})$ \\
\hline 8 & 95.5 & $6.51(\mathrm{~d}, J=2.2 \mathrm{~Hz})$ & $6^{\prime \prime}$ & 61.2 & $\begin{array}{l}3.58(\mathrm{dd}, J=12.0,5.8 \mathrm{~Hz}) \\
3.80(\mathrm{dd}, J=12.0,1.9 \mathrm{~Hz})\end{array}$ \\
\hline 9 & 157.9 & - & & & \\
\hline 10 & 106.3 & - & & & \\
\hline $1^{\prime}$ & 131.3 & - & & & \\
\hline $2^{\prime}, 6^{\prime}$ & 127.0 & $8.09(\mathrm{dd}, J=8.2,1.4 \mathrm{~Hz})$ & & & \\
\hline $3^{\prime}, 5^{\prime}$ & 129.7 & $7.54-7.64$ & & & \\
\hline $4^{\prime}$ & 132.7 & $7.54-7.64$ & & & \\
\hline
\end{tabular}


<smiles>[R]Oc1cc2oc(-c3ccc([R2])c([R])c3)cc(=O)c2c(O)c1[R3]</smiles>

\begin{tabular}{|c|c|c|c|c|c|c|}
\hline Peak & Flavones & $\mathbf{R} \mathbf{1}$ & $\mathbf{R 2}$ & $\mathbf{R 3}$ & $\mathbf{R} 4$ & R5 \\
\hline 1 & 2"-O-pentosyl-6- $C$-hexosyl-luteolin & $\mathrm{OH}$ & $\mathrm{OH}$ & Hex-Pent & $\mathrm{H}$ & $\mathrm{H}$ \\
\hline 2 & 2"-O-pentosyl-8-C-hexosyl-luteolin & $\mathrm{OH}$ & $\mathrm{OH}$ & $\mathrm{H}$ & $\mathrm{H}$ & Hex-Pent \\
\hline 4 & $2 "$-O-pentosyl-8-C-hexosyl-apigenin & $\mathrm{H}$ & $\mathrm{OH}$ & $\mathrm{H}$ & $\mathrm{H}$ & Hex-Pent \\
\hline 5 & 2"-O-pentosyl-6-C-hexosyl-apigenin & $\mathrm{H}$ & $\mathrm{OH}$ & Hex-Pent & $\mathrm{H}$ & $\mathrm{H}$ \\
\hline 6 & $\begin{array}{l}\text { 6"-O-(3-hydroxy-3-methylglutaroyl)-2"-O- } \\
\text { pentosyl-8-C-hexosyl-luteolin }\end{array}$ & $\mathrm{OH}$ & $\mathrm{OH}$ & $\mathrm{H}$ & $\mathrm{H}$ & $\begin{array}{c}\text { Hex-Pent- } \\
\text { HMG }\end{array}$ \\
\hline 7 & $\begin{array}{l}\text { 6"-O-(3-hydroxy-3-methylglutaroyl)-2"-O- } \\
\text { pentosyl-8-C-hexosyl-apigenin }\end{array}$ & $\mathrm{H}$ & $\mathrm{OH}$ & $\mathrm{H}$ & $\mathrm{H}$ & $\begin{array}{c}\text { Hex-Pent- } \\
\text { HMG }\end{array}$ \\
\hline 9 & Chrysin-7-O-glucoside & $\mathrm{H}$ & $\mathrm{H}$ & $\mathrm{H}$ & Glc & $\mathrm{H}$ \\
\hline
\end{tabular}

Hex- Hexose; Pent- Pentose; Glc- Glucose; HMG- 3-hydroxy-3-methylglutaroyl

Fig. 3. Proposed structures for flavones identified in the ethanolic extract of Cytisus multiflorus.

The luteolin derivative found in fraction 6 (molecular ion at $\mathrm{m} / \mathrm{z}$ 723 or $m / z 725$, in negative or positive ion modes, respectively) was structurally related to the previous ones. Indeed, the $\mathrm{MS}^{2}$ of the ion at $m / z 723$ (negative mode) showed a base peak ion at $m /$ $z 579$, which corresponds to one of the 2 "-O-pentosyl-C-hexosylluteolin isomers described above (fraction 1 or 2). This product ion was formed by the loss of $144 \mathrm{Da}$, and other ions in the $\mathrm{MS}^{2}$ spectrum were formed by the loss of $62 \mathrm{Da}$ (at $\mathrm{m} / \mathrm{z} 661$ ) and $102 \mathrm{Da}$ (at $\mathrm{m} / \mathrm{z} 621$ ). Luteolin derivatives containing a $144 \mathrm{Da}$ moiety were previously described by Ferreres, Sousa, et al. (2007) in Passiflora genus, although at that time, these authors have not proposed a structural feature for that unit. Yet, the same fragmentation pattern ( $-62 \mathrm{Da},-102 \mathrm{Da}$ and $-144 \mathrm{Da}$ ) has been previously assigned to 3-hydroxy-3-methylglutaroyl flavonoid glycosides in Citrus bergamia (Di Donna et al., 2009) and in Oxytropis racemosa plant of Fabaceae family (Song et al., 2010). Thus, based on that data, these results suggest the existence of a 3-hydroxy-3-methylglutaroyl derivative of $2^{\prime \prime}-O$-pentosyl-C-hexosyl-luteolin in $C$. multiflorus. To our knowledge, luteolin derivative compounds containing a 3-hydroxy-3-methylglutaroyl moiety have never been reported in Fabaceae and thus, further studies are needed in order to elucidate the specific linkage position of the 3-hydroxy-3-methylglutaroyl moiety to the phenolic skeleton. In this context, the structure proposed in Fig. 3 should only be regarded as an example.

More common glycosyl-luteolin derivatives (MW $448 \mathrm{Da}$ ) occurred as minor components of the ethanolic extract and were detected in fractions 3,6 and 7. The assignment of these compounds to orientin (fraction 3) (Kazuno et al., 2005), luteolin-5-O-glucoside (fraction 6) and luteolin-7-O-glucoside (fraction 7) (Rauter et al., 2009), which was based on the HPLC-DAD and MS ${ }^{\mathrm{n}}$ data, will not be discussed in detail, as they were previously described to occur in Fabaceae.

\subsubsection{Apigenin derivatives}

New apigenin derivatives in $C$. multiflorus belonged to the same group as those of luteolin derivatives, i.e., the 2 "-O-glycosyl-C-glycosyl-flavones. Indeed, besides apigenin (fraction 11) and
apigenin-7-O-glucoside (fraction 8), the derivatives of this flavone detected in fractions 4,5 and 7 had comparable fragmentation pathway to that described for $2^{\prime \prime}$-O-glycosyl-C-glycosyl-luteolin derivatives. Namely, the MS ${ }^{2}$ of the abundant molecular ion at $m /$ $z 563$ (fractions 4 and 5) showed a base peak formed by the loss of $150 \mathrm{Da}$ (ion at $\mathrm{m} / \mathrm{z} 413$ ), indicating the presence of an $O$-pentosyl group (Ferreres, Gil-Izquierdo, et al., 2007). Moreover, the detection of the product ion $[\mathrm{M}-\mathrm{H}-120]^{-}$(ion at $m / z 443$ ) in the $\mathrm{MS}^{2}$ spectrum, as also the ion at $m / z 293$ in the $\mathrm{MS}^{3}$ spectrum (representing the apigenin aglycone $+41-18 \mathrm{Da}$ ), corroborated the presence of $2^{\prime \prime}-O$-pentosyl-C-hexosyl-apigenin isomers in fractions 4 and 5 (Ferreres, Gil-Izquierdo, et al., 2007). Overall, the MS data suggested the presence of $2^{\prime \prime}-O$-pentosyl-8-C-hexosyl-apigenin (2"-O-pentosyl-vitexin) and $2^{\prime \prime}-O$-pentosyl-6-C-hexosyl-apigenin ( 2 "-O-pentosyl-isovitexin) in those fractions (structures represented in Fig. 3). Attending that 8-C-glucosyl-apigenin elutes before 6 -C-glucosyl-apigenin under HPLC reverse phase conditions (Kazuno et al., 2005; Pereira et al., 2005; Piccinelli et al., 2008), compounds of MW $564 \mathrm{Da}$ in fractions 4 and 5 were respectively assigned to $2^{\prime \prime}-O$-pentosyl-vitexin and $2^{\prime \prime}-O$-pentosyl-isovitexin. To our knowledge, these compounds were here detected for the first time in Fabaceae family.

Similarly to that described for luteolin derivatives, the ethanolic extract of $C$. multiflorus also contained one 3-hydroxy-3-methylglutaroyl derivative of $2^{\prime \prime}-O$-pentosyl-C-hexosyl-apigenin (MW $708 \mathrm{Da})$. The base peak in the $\mathrm{MS}^{2}$ spectrum in the negative mode $\left([\mathrm{M}+\mathrm{H}]^{-}\right.$at $m / z$ 707) corresponded to one $2^{\prime \prime}-O$-pentosyl-C-hexosyl-apigenin moiety (ion at $m / z 563$ ), and this latter ion had a similar fragmentation pattern to that of the C-glycosyl isomer. As described before for the luteolin derivative in fraction 6 (MW $724 \mathrm{Da}$ ), the presence of an 3-hydroxy-3-methylglutaric acid moiety was proposed based on literature data (Di Donna et al., 2009; Song et al., 2010). Apigenin derivatives containing a 144 Da moiety were also previously described by Ferreres, Sousa, et al. (2007) in Passiflora genus, although no structural feature was proposed. As for the luteolin derivative, the proposed structure of the apigenin derivative detected in fraction 7 (Fig 3 ) is based on the literature 
Table 3

Quantification of the identified compounds in the ethanolic extract of Cytisus multiflorus.

\begin{tabular}{clr}
\hline $\begin{array}{c}\text { Number } \\
\text { fraction }\end{array}$ & Compound & $\begin{array}{c}\mathrm{mg} / \mathrm{g} \\
\text { dried } \\
\text { plant }\end{array}$ \\
\hline 1 & $2^{\prime \prime}$-O-pentosyl-6-C-hexosyl-luteolin & $3.3 \pm 0.5$ \\
2 & $2^{\prime \prime}-O$-pentosyl-8-C-hexosyl-luteolin & $3.5 \pm 0.3$ \\
3 & Orientin & $0.8 \pm 0.1$ \\
4 & $2^{\prime \prime}-O$-pentosyl-8-C-hexosyl-apigenin & $0.5 \pm 0.1$ \\
5 & 2"-O-pentosyl-6-C-hexosyl-apigenin & $0.9 \pm 0.1$ \\
6 & Rutin & $4.5 \pm 0.7$ \\
7 & 6"-O-(3-hydroxy-3-methylglutaroyl)-2"-O-pentosyl- & $3.6 \pm 0.7$ \\
& C-hexosyl-apigenin & \\
8 & Apigenin-7-O- glucoside & $0.8 \pm 0.1$ \\
9 & Chrysin-7-O- $\beta$-D-glucopyranoside & $15.9 \pm 2.3$ \\
10 & Dihydroxyflavone isomer of chrysin & $7.0 \pm 1.3$ \\
11 & Apigenin & $0.5 \pm 0.1$ \\
12 & Chrysin & $0.5 \pm 0.1$ \\
Total & & $41.8 \pm 3.0$ \\
\hline
\end{tabular}

data reported for the Fabaceae family (Liu, Liu, Liu, Hou, \& Mabry, 1994), although further studies are needed in order to confirm that hypothesis.

\subsubsection{Quercetin derivatives}

Besides the above described flavones, the ethanolic extract of $C$. multiflorus also contained two common derivatives of the flavonol quercetin. According to the HPLC-DAD the ESI-MS ${ }^{\mathrm{n}}$ figures, and also the comparison to the literature data, flavonols were assigned to rutin (MW $610 \mathrm{Da}$ in fraction 6) and quercetin-3-O-glucoside (MW 464 Da in fraction 7), which have been previously described in Fabaceae family (Raja et al., 2007).

\subsection{Quantification of phenolic compounds by HPLC-DAD}

The quantified phenolic compounds in the ethanolic extract of C. multiflorus (Table 3) accounted for $41.8 \pm 3.0 \mathrm{mg} / \mathrm{g}$ dried plant, which is a close value to that obtained by the Folin-Ciocalteu method $(44.7 \pm 4.0 \mathrm{mg} / \mathrm{g}$ dried plant). The extract was shown to be mostly rich in chrysin derivatives, in particular the flavone chrysin-7-O- $\beta$-D-glucopyranoside. This latter component, together with the dihydroxyflavone (chrysin isomer in fraction 10), accounted for approximately $50 \%$ of the extract total phenolic content. Besides those two compounds, the flavones $2^{\prime \prime}-O$-pentosyl-6-C-hexosylluteolin, 2"-O-pentosyl-8-C-hexosyl-luteolin and 6"-O-(3-hydroxy-3-methylglutaroyl)-2"-O-pentosyl-C-hexosyl-apigenin, as well as flavonol rutin can also be pointed as occurring in moderate concentrations in the ethanolic extract of $C$. multiflorus.

\section{Conclusions}

The ethanolic extract obtained from flowers of $C$. multiflorus was here described in detail for the first time, by means of HPLC-DAD, ESI-MS and MS ${ }^{\mathrm{n}}$ analyses and NMR assays. The main compound in the phenolic extract of the flowers of this plant was chrysin-7-O- $\beta$-Dglucopyranoside, but it also contained considerable amounts of rutin, a dihydroxyflavone isomer of chrysin, $2^{\prime \prime}-0$-pentosyl-6-C-hexosylluteolin, 2"-O-pentosyl-8-C-hexosyl-luteolin and 6"-O-(3-hydroxy-3methylglutaroyl)-2"-O-pentosyl-C-hexosyl-apigenin, which are not commonly found in the Fabaceae family. Moreover, other unusual phenolic compounds found in minor amounts in the ethanolic extract of $C$. multiflorus were identified as 2"-O-pentosyl-6-C-hexosyl-apigenin, $2^{\prime \prime}$ $O$-pentosyl-8-C-hexosyl-apigenin and 6"-O-(3-hydroxy-3-methylglutaroyl)-2"-O-pentosyl-C-hexosyl-luteolin. Overall, the present work is a valuable contribution for the phenolic elucidation of the Cytisus genus and of the Fabaceae family.

\section{Acknowledgements}

The authors acknowledge the financial support provided by the FCT to CERNAS and of the FCT as well as FSE (III Quadro Comunitário de Apoio), to QOPNA and REDE/1504/REM/2005 (that concerns the Portuguese Mass Spectrometry Network). Olívia R Pereira was supported by a PhD grant (SFRH/PROTEC/49600/2009). The authors are also grateful to Eng Jorge Sá Morais for technical support in the HPLC analysis.

\section{References}

Castro, V. (1998). Chromium in a series of Portuguese plants used in the herbal treatment of diabetes. Biological Trace Element Research, 62, 101-106.

Castro, V. (2001). Chromium and zinc in a series of plants used in Portugal in the herbal treatment of non-insulinized diabetes. Acta Alimentaria, 30, 333-342.

Ciudad, A. G., Santos, B. F., de Aldana, B. R. V., Zabalgogeazcoa, I., Gutierrez, M. Y., \& Criado, B. G. (2004). Use of near infrared reflectance spectroscopy to assess forage-quality of a Mediterranean shrub. Communications in Soil Science and Plant Analysis, 35, 665-678.

Cristofolini, G., \& Conte, L. (2002). Phylogenetic patterns and endemism genesis in Cytisus Desf. (Leguminosae-Cytiseae) and related genera. Israel Journal of Plant Sciences, 50, 37-50.

Cristofolini, G., \& Troia, A. (2006). A reassessment of the sections of the genus Cytisus Desf. (Cytiseae, Leguminosae). Taxon, 55, 733-746.

Cuyckens, H., \& Claeys, M. (2004). Mass spectrometry in the structural analysis of flavonoids. Journal of Mass Spectrometry, 39, 1-15.

Di Donna, L., De Luca, G., Mazzotti, F., Napoli, A., Salerno, R., Taverna, D., et al. (2009). Statin-like Principles of Bergamot Fruit (Citrus bergamia): Isolation of $3-$ Hydroxymethylglutaryl Flavonoid Glycosides. Journal of Natural Products, 72, $1352-1354$.

Di Giorgio, C., Delmas, F., Tueni, M., Cheble, E., Khalil, T., \& Balansard, G. (2008). Alternative and complementary antileishmanial treatments: Assessment of the antileishmanial activity of 27 Lebanese plants, including 11 endemic species. Journal of Alternative and Complementary Medicine, 14, 157-162.

El Antri, A., Messouri, I., Tlemcani, R. C., Bouktaib, M., El Alami, R., El Bali, B., et al. (2004). Flavone glycosides from Calycotome villosa subsp. intermedia. Molecules, 9, 568-573.

Ferreira, D., Guyot, S., Marnet, N., Delgadillo, I., Renard, C., \& Coimbra, M. A. (2002). Composition of phenolic compounds in a Portuguese pear (Pyrus communis L. var. S. Bartolomeu) and changes after sun-drying. Journal of Agricultural and Food Chemistry, 50, 4537-4544.

Ferreres, F., Gil-Izquierdo, A., Andrade, P. B., Valentao, P., \& Tomas-Barberan, F. A. (2007a). Characterization of C-glycosyl flavones O-glycosylated by liquid chromatography-tandem mass spectrometry. Journal of Chromatography A, $1161,214-223$.

Ferreres, F., Sousa, C., Valentao, P., Andrade, P. B., Seabra, R. M. \& Gil-Izquierdo, A. (2007b). New C-deoxyhexosyl flavones and antioxidant properties of Passiflora edulis leaf extract. Journal of Agricultural and Food Chemistry, 55, 10187-10193.

Gião, M. S., Gonzalez-Sanjose, M. L., Rivero-Perez, M. D., Pereira, C. I., Pintado, M. E., \& Malcata, F. X. (2007). Infusions of Portuguese medicinal plants: Dependence of final antioxidant capacity and phenol content on extraction features. Journal of the Science of Food and Agriculture, 87, 2638-2647.

Han, J., Ye, M., Qiao, X., Xu, M., Wang, B.-R., \& Guo, D.-A. (2008). Characterization of phenolic compounds in the Chinese herbal drug Artemisia annua by liquid chromatography coupled to electrospray ionization mass spectrometry. Journal of Pharmaceutical and Biomedical Analysis, 47, 516-525.

Hanganu, D., Vlase, L., \& Olah, N. (2010a). LC/MS analysis of isoflavones from Fabaceae species extracts. Farmacia, 58, 177-183.

Hanganu, D., Vlase, L., \& Olah, N. (2010b). Phytochemical analysis of isoflavons from some Fabaceae species extracts. Notulae Botanicae Horti Agrobotanici ClujNapoca, 38, 57-60.

Ioset, J. R., Urbaniak, B., Ndjoko-Ioset, K., Wirth, J., Martin, F., Gruissem, W., et al. (2007). Flavonoid profiling among wild type and related GM wheat varieties. Plant Molecular Biology, 65, 645-654.

Kazuno, S., Yanagida, M., Shindo, N., \& Murayama, K. (2005). Mass spectrometric identification and quantification of glycosyl flavonoids, including dihydrochalcones with neutral loss scan mode. Analytical Biochemistry, 347, 182-192.

Liu, M., Liu, Q., Liu, Y. L., Hou, C. Y., \& Mabry, T. J. (1994). An acylated flavone CGlycoside from Glycyrrhiza eurycarpa. Phytochemistry, 36, 1089-1090.

Luis, A., Domingues, F., Gil, C., \& Duarte, A. P. (2009). Antioxidant activity of extracts of Portuguese shrubs: Pterospartum tridentatum, Cytisus scoparius and Erica spp. Journal of Medicinal Plants Research, 3, 886-893.

Nirmal, J., Babu, C. S., Harisudhan, T., \& Ramanathan, M. (2008). Evaluation of behavioural and antioxidant activity of Cytisus scoparius Link in rats exposed to chronic unpredictable mild stress. BMC Complementary and Alternative Medicine, 8,8 .

Pereira, C. A. M., Yariwake, J. H., \& McCullagh, M. (2005). Distinction of the Cglycosylflavone isomer pairs orientin/isoorientin and vitexin/isovitexin using HPLC-MS exact mass measurement and in-source CID. Phytochemical Analysis, 16, 295-301. 
Piccinelli, A. L., Mesa, M. G., Armenteros, D. M., Alfonso, M. A., Arevalo, A. C., Campone, L., et al. (2008). HPLC-PDA-MS and NMR characterization of Cglycosyl flavones in a hydroalcoholic extract of Citrus aurantifolia leaves with antiplatelet activity. Journal of Agricultural and Food Chemistry, 56, 1574-1581.

Raja, S., Ahamed, K., Kumar, V., Mukherjee, K., Bandyopadhyay, A., \& Mukherjee, P. K. (2007). Antioxidant effect of Cytisus scoparius against carbon tetrachloride treated liver injury in rats. Journal of Ethnopharmacology, 109, 41-47.

Rauter, A. P., Martins, A., Lopes, R., Ferreira, J., Serralheiro, L. M., Araujo, M. E., et al. (2009). Bioactivity studies and chemical profile of the antidiabetic plant Genista tenera. Journal of Ethnopharmacology, 122, 384-393.

Regos, I., Urbanella, A., \& Treutter, D. (2009). Identification and Quantification of Phenolic Compounds from the Forage Legume Sainfoin (Onobrychis viciifolia). Journal of Agricultural and Food Chemistry, 57, 5843-5852.

Rodriguez-Riano, T., Ortega-Olivencia, A., \& Devesa, J. A. (1999). Reproductive phenology in three Genisteae (Fabaceae) shrub species of the W Mediterranean Region. Nordic Journal of Botany, 19, 345-354.

Rodriguez-Riano, T., Ortega-Olivencia, A., \& Devesa, J. A. (2004). Reproductive biology in Cytisus multiflorus (Fabaceae). Annales Botanici Fennici, 41, 179-188.

Rodriguez-Riano, T., Valtuena, F. J., \& Ortega-Olivencia, A. (2006). Megasporogenesis, megagametogenesis and ontogeny of the aril in Cytisus striatus and C-multiflorus (Leguminosae: Papilionoideae). Annals of Botany, 98, 777-791.

Siegel, R. K. (1976). Herbal intoxication - Psychoactive effects from herbal cigarettes, tea, and capsules. Jama-Journal of the American Medical Association, 236, 473-476.

Singleton, V. L., \& Rossi, J. A. (1965). Colorimetry of total phenolics with phosphomolybdic-phosphotungstic acid reagents. American Journal of Enology and Viticulture, 16, 144-158.

Song, S. A., Zheng, X. P., Liu, W. D., Du, R. F., Bi, L. F., \& Zhang, P. C. (2010). 3Hydroxymethylglutaryl Flavonol Glycosides from a Mongolian and Tibetan Medicine, Oxytropis racemosa. Chemical \& Pharmaceutical Bulletin, 58, 1587-1590.

Sundararajan, R., Haja, N. A., Venkatesan, K., Mukherjee, K., Saha, B. P., Bandyopadhyay, A., et al. (2006). Cytisus scoparius link-a natural antioxidant. BMC Complementary and Alternative Medicine, 6, 8.

Ye, M. Yan, Y. N., \& Guo, D. A. (2005). Characterization of phenolic compounds in the Chinese herbal drug Tu-Si-Zi by liquid chromatography coupled to electrospray ionization mass spectrometry. Rapid Communications in Mass Spectrometry, 19, 1469-1484. 


\subsection{Phenolic Constituents of Lamium album: Focus on ISOSCUTELLAREIN DERIVATIVES}

Lamium album L., commonly known as white dead nettle, is a Mediterranean perennial herb consumed as food ingredient, in food supplements and in the form of tea. Despite the plant has been traditionally used for the treatment of several diseases, a detailed knowledge of its phenolic constituents, as well as their content in the plant, is still missing. In this work, a detailed phenolic characterization was performed for a purified ethanolic extract obtained from the aerial parts of $L$. album.

The extract was mainly rich in the two phenylethanoids verbascoside $(233.7 \pm 13.6 \mathrm{mg} / \mathrm{g}$ extract) and isoverbascoside (39.2 $\pm 5.6 \mathrm{mg} / \mathrm{g}$ extract), corresponding approximately to half of the total quantified phenolics. Besides these, the extract also contained uncommon bioactive phenolic compounds herein detected for the first time in the Lamium genus, called isoscutellarein derivatives. These compounds accounted for almost $30 \%$ of the total quantified phenolics in the extract. They included the isoscutellarein-7-O-allosyl $(1 \rightarrow 2)$ glucoside, its $O$-methyl derivative, three acetyl derivatives of isoscutellarein-O-allosyl glucoside and one acetylated form of $O$ methylisoscutellarein-7-O-allosyl $(1 \rightarrow 2)$ glucoside. The main isoscutellarein derivative was assigned to isoscutellarein-7-O-(6-O-acetyl- $\beta$-allosyl) $(1 \rightarrow 2)-\beta$-glucoside (accounting for $37.4 \pm 4.4 \mathrm{mg} / \mathrm{g}$ extract), as confirmed by NMR experiments. In turn, the flavones apigenin-7-O-glucoside, luteolin-7-O-glucoside, apigenin-7-O-rutinoside and the flavanone naringenin-7-O-rutinoside were detected as minor components of the extract. In conclusion, this work details the chemical characterization of the L. album, also suggesting that this species is an important dietary source of natural antioxidants. It is also expected that the herein gathered data will stimulate further search in order to elucidate the possible roles of $L$. album phenolics on the benefits of the plant.

The results obtained in this section of the Doctoral Thesis have been used to write the manuscript entitled "Phenolic constituents of Lamium album: focus on isoscutellarein derivatives" which is published in Food Research International. 


\title{
Phenolic constituents of Lamium album: Focus on isoscutellarein derivatives
}

\author{
Olívia R. Pereira ${ }^{\mathrm{a}, \mathrm{b}}$, Maria R.M. Domingues ${ }^{\mathrm{c}}$, Artur M.S. Silva ${ }^{\mathrm{c}}$, Susana M. Cardoso ${ }^{\mathrm{a}, \mathrm{d}, *}$ \\ ${ }^{a}$ CERNAS - Escola Superior Agrária, Instituto Politécnico de Coimbra, Bencanta, 3040-316 Coimbra, Portugal \\ b Departamento de Tecnologias de Diagnóstico e Terapêutica, Escola Superior de Saúde, Instituto Politécnico de Bragança, Av. D. Afonso V, 5300-121 Bragança, Portugal \\ c QOPNA - Departamento de Química, Universidade de Aveiro, 3810-193 Aveiro, Portugal \\ d CIMO - Escola Superior Agrária, Instituto Politécnico de Bragança, Campus de Santa Apolónia - Apartado 1038-5301-854, Bragança, Portugal
}

\section{A R T I C L E I N F O}

Article history:

Received 9 January 2012

Accepted 30 April 2012

Available online 11 May 2012

\section{Keywords:}

Lamium album

Verbascoside

Isoverbascoside

Isoscutellarein derivatives

High performance liquid chromatography

Electrospray ionization-mass spectrometry

\begin{abstract}
A B S T R A C T
Lamium album L. is an edible plant which is consumed raw or cooked, in particular in the Mediterranean and surrounding areas. It is also consumed as tea infusions and as a main component of food supplements, because of its pharmacological effects. Despite being consumed by humans for centuries, the chemical composition of $L$. album $\mathrm{L}$. is far from being understood. In this study, a purified ethanolic extract (PEEL) was prepared and further analyzed by high performance liquid chromatography and electrospray mass spectrometry. Overall, verbascoside accounted for approximately half of the phenolic content of the extract, but this also contained other bioactive phenolic compounds herein detected for the first time in the genus, namely isoscutellarein derivatives. The latter included isoscutellarein-7-O-allosyl $(1 \rightarrow 2)$ glucoside, its $O$-methyl derivative, three acetyl derivatives of isoscutellarein- 0 allosyl glucoside and one acetylated form of 0 -methylisoscutellarein-7-O-allosyl $(1 \rightarrow 2)$ glucoside. From those, the main isoscutellarein derivative was assigned to isoscutellarein-7-O-(6-O-acetyl- $\beta$-allosyl $)(1 \rightarrow 2)-\beta$-glucoside, as confirmed by NMR. Altogether, isoscutellarein derivatives accounted for almost $30 \%$ of PEEL phenolics. Since verbascoside and isoscutellarein derivatives are main components of $L$. album L. ethanolic extract, their possible association to the health benefits of the plant is discussed.
\end{abstract}

(c) 2012 Elsevier Ltd. All rights reserved.

\section{Introduction}

The genus Lamium L. (Family: Lamiaceae alt. Labiatae) comprises about 40 annual or perennial herb species native to the Old World, distributed in Europe, Asia and Africa.

Lamium album L. is a perennial herb commonly known as white dead nettle that has been used as emergency or famine food, particularly during the specific decades of starvation as an alternative nourishment in different countries such as Europe, China and Japan (Baranov, 1967; Luczaj, 2008; Sturtevants, 1919; Turner et al., 2011). In modern times, L. album L. is mainly consumed in the Mediterranean and surrounding areas for confection of local dishes (Heinrich, Müller, \& Galli, 2006). In fact, the young shoots, leaves and flowers of this plant are edible and consumed raw or cooked as a vegetable. The plant is also commonly used as an ingredient in several dishes including omelets, stews and roasts (Clifford, 2001). Moreover, white dead nettle is the base ingredient for important vegetarian dishes such as the "White Dead Nettle Frittata", "White Dead Nettle, Feta and Watermelon Salad" and the “Deadnettle soup" (Celnet, 2005; Harford, 2007).

\footnotetext{
* Corresponding author at: CERNAS - Escola Superior Agrária, Instituto Politécnico de Coimbra, Bencanta, 3040-316 Coimbra, Portugal. Tel.: +351 239 802940; fax: + 351273239802979 .

E-mail address: scardoso@esac.pt (S.M. Cardoso).
}

L. album L. is also used in teas and in food supplement preparations, the consumption of which is primarily associated to the plant health benefits. In particular, the consumption of food supplements enriched in L. album L. extracts are claimed to detoxify the organism, to prevent menstrual disorders, abdominal inflammation and musculoskeletal diseases (Xu, 2008) and to improve fat metabolism (Ninomiya et al., 2006).

Besides the above applications, the flowers of L. album L. are attractive to bees and other pollinating insects and hence, are frequently used in honey production (Denisow \& Bozek, 2008; Mihaly Cozmuta, Bretan, Mihaly Cozmuta, Nicula, \& Peter, in press).

During the last decades food health attributes have become an important issue of concern for consumers, clearly influencing their choices. In parallel, the search for food constituents related to health properties has incredibly raised. This provides the base knowledge to understand the beneficial properties of a particular food product and further stimulate consumers' interest in it. In the particular case of L. album L., the phenolic compounds have been closely associated with the antioxidant properties of the plant (Matkowski \& Piotrowska, 2006; Valyova, Dimitrova, Ganeva, Mihova Kapchina-Toteva, \& Petkova Yordanova, 2011), as well as to its remaining health benefits (Paduch et al., 2008; Paduch, Wójciak-Kosior, \& Matysik, 2007).

In this way, several L. album L. phenolic compounds have already been detected, which include the flavonoids quercetin, quercetin-3-Oglucoside, rutin, isoquercitrin, kaempferol-3-O-glucoside and tiliroside, 
the phenolic acids protocatechuic, chlorogenic, vanillic and caffeic and the phenylpropanoid glycoside ester derivatives lamalboside, acteoside and isoacteoside (Budzianowski \& Skrzypczak, 1995; Paduch et al., 2007; Yalcin \& Kaya, 2006). However despite that information, a detailed knowledge of the L. album L. phenolic constituents, as well as their content in the plant, is still missing. Hence, these two topics will be herein described in detail.

\section{Experimental}

\subsection{Chemicals}

The phenolic standards verbascoside, apigenin-7-0-glucoside, luteolin-7-0-glucoside and naringenin-7-O-glucoside were obtained from Extrasynthese (Genay Cedex, France). Gallic acid was obtained from Sigma Chemical Co (St Louis, MO, USA), while Folin-Ciocalteu reagent, $\mathrm{Na}_{2} \mathrm{CO}_{3}$, formic acid and ethanol were purchased from Panreac (Barcelona, Spain). n-Hexane, methanol and acetonitrile with HPLC purity were purchased from Lab-Scan (Lisbon, Portugal). Water was treated in a Milli-Q water purification system (TGI Pure Water Systems, USA). DMSO- $\mathrm{d}_{6}$ containing $0.03 \%$ of TMS was obtained from CortecNet (Paris, France).

\subsection{Plant material}

The $L$. album were purchased as a mixture of flowers, leaves and stems from O SEGREDO DA PLANTA - Produtos Naturais e Biológicos, Lda. (Seixal, Portugal). The plants have been cultivated under an organic regime and after collection, its aerial parts (flowers, leaves and stems) were dried in a ventilated incubator at 20 to $35^{\circ} \mathrm{C}$ for 3 to 5 days.

\subsection{Extraction of phenolic compounds}

The aerial parts (flowers, leaves and stems) of $L$ album ( $5 \mathrm{~g}$ ) were ground together and defatted three times with $150 \mathrm{~mL}$ of $n$-hexane. The residue was extracted with $150 \mathrm{~mL}$ of an $80 \%$ ethanol solution $(v / v)$ at room temperature, for $1 \mathrm{~h}$ and the resulting mixture was filtered. The residue was similarly re-extracted five times and the filtrated solutions were combined, concentrated, frozen at $-20{ }^{\circ} \mathrm{C}$ and freeze-dried. The dried extract (ethanolic extract) of $L$. album was stored under vacuum, in a desiccator in dark, for subsequent use (Pereira, Silva, Domingues, \& Cardoso, 2012). This procedure was performed in triplicate.

\subsection{Purification of phenolic compounds}

The ethanolic extracts were further purified for phenolic enrichment. For that, approximately $0.4 \mathrm{~g}$ of each ethanolic extract was dissolved in $15 \mathrm{~mL}$ of water and eluted in three Strata SPE C18-E cartridges (2 g, Waters, Milford, MA, USA). The cartridges were then washed three times with $30 \mathrm{~mL}$ of water, and the phenolic compounds were recovered by elution with $20 \mathrm{~mL}$ of methanol. The residue was concentrated, frozen at $-20^{\circ} \mathrm{C}$ and freeze-dried to give the purified ethanolic extract (PEEL) (Pereira et al., 2012).

\subsection{Quantification of total phenolic compounds}

Total concentration of phenolic compounds was determined according to the adapted Folin-Ciocalteu colorimetric method (Singleton \& Rossi, 1965). A mixture of $250 \mu \mathrm{L}$ of Folin-Ciocalteu reagent and $0.5 \mathrm{~mL}$ plant extract solution $(0.4 \mathrm{mg} / \mathrm{mL})$ was prepared. After $3 \mathrm{~min}, 1 \mathrm{~mL}$ of $\mathrm{Na}_{2} \mathrm{CO}_{3}(200 \mathrm{~g} / \mathrm{L})$ and $3.25 \mathrm{~mL}$ of milliQ water were added. The mixture was homogenized and incubated for $10 \mathrm{~min}$ at $70{ }^{\circ} \mathrm{C}$, and then kept at room temperature for $30 \mathrm{~min}$. The absorbance was measured at $700 \mathrm{~nm}$ and the amount of total phenolic compounds was expressed as gallic acid equivalent ( $\mathrm{mg} \mathrm{GAE}$ )/g dried weight of plant material using a calibration curve of gallic acid as standard ( 5 to
$37.5 \mu \mathrm{g} / \mathrm{mL}$ ). This procedure was performed at least in duplicate for the three PEEL samples.

\subsection{HPLC apparatus and chromatographic conditions}

The HPLC analysis was performed on a Varian 9010 separation module equipped with a PDA Varian Prostar detector and data acquisition and remote control of the HPLC system were done by Varian Star chromatography Workstation ${ }^{\circledR}$ (Lake Forest, CA, USA) software. The column used was a $250 \mathrm{~mm} \times 4 \mathrm{~mm}$ id, $5 \mu \mathrm{m}$ bead diameter, endcapped Nucleosil C18 (Macherey-Nagel) and its temperature was maintained at $30^{\circ} \mathrm{C}$.

Gradient elution was carried out with a mixture of $0.1 \%(v / v)$ of formic acid in water (solvent A) and acetonitrile (solvent B), which were degassed and filtered before use. The solvent gradient consisted of a series of linear gradients, starting from 10 to $20 \%$ of solvent B over $6 \mathrm{~min}, 20$ to $25 \%$ of solvent B over $6 \mathrm{~min}, 25$ to $40 \%$ over $30 \mathrm{~min}$, increasing to $45 \%$ at $50 \mathrm{~min}$ and to $100 \%$ of solvent B over 5 min decreasing to $10 \%$ of solvent B after 5 min followed by the return to the initial conditions. The flow rate used was $1 \mathrm{~mL} / \mathrm{min}$. For the HPLC analysis, the samples (10 mg) were dissolved in $2 \mathrm{~mL}$ of methanol, filtered through a $0.2 \mu \mathrm{m}$ Nylon membrane (Whatman) and $10 \mu \mathrm{L}$ of each solution was injected. The UV-vis spectra were recorded between 220 and $500 \mathrm{~nm}$ and the chromatographic profiles were recorded at $340 \mathrm{~nm}$.

\subsection{Identification and quantification of the phenolic compounds}

Identification of the compounds was performed by HPLC-DAD and ESI-MS analysis. The compounds were firstly identified according to the retention time and UV-vis spectra of the HPLC eluting peaks. After three manual collections, further characterization of the eluted compounds was accomplished by electrospray ionization mass spectrometry (ESI-MS and ESI-MS ${ }^{\mathrm{n}}$ ) using a Linear Ion trap LXQ mass spectrometer (ThermoFinnigan, San Jose, CA, USA), following the general procedure previously described (Pereira et al., 2012). Moreover, the most abundant isoscutellarein derivative (fraction 9) was further analyzed by NMR spectroscopy. To accomplish that, approximately $3 \mathrm{mg}$ of freeze-dried material of this HPLC fraction was dissolved in DMSO$\mathrm{d}_{6}$ and the ${ }^{1} \mathrm{H}$ and ${ }^{13} \mathrm{C}$ NMR spectra were recorded at $298 \mathrm{~K}$ on a Bruker Avance 500 spectrometer operating at $500.13 \mathrm{MHz}$ and $125.77 \mathrm{MHz}$, respectively. The phase sensitive ${ }^{1} \mathrm{H}$-detected $\left({ }^{1} \mathrm{H}^{13} \mathrm{C}\right)$ gHSQC (heteronuclear single quantum coherence, using gradient pulses for selection) spectrum was recorded with 216 transients over 256 increments (zero-filled to 512) and $2 \mathrm{~K}$ data points with spectral widths of $4500 \mathrm{~Hz}$ in $\mathrm{F}_{2}$ and $20 \mathrm{kHz}$ in $\mathrm{F}_{1}$. The repetition time was $1.9 \mathrm{~s}$. A cosine multiplication was applied in both dimensions. The delays were adjusted according to a coupling constant ${ }^{1} \mathrm{~J}(\mathrm{CH})$ of $147 \mathrm{~Hz}$. The gHMBC (heteronuclear multiple quantum coherence, using gradient pulses for selection) spectrum was recorded with 240 transients over 256 increments (zero-filled to $1 \mathrm{~K}$ ) and $2 \mathrm{~K}$ data points with spectral widths of $4500 \mathrm{~Hz}$ in $F_{2}$ and $25 \mathrm{kHz}$ in $F_{1}$. The repetition time was $1.9 \mathrm{~s}$. A sine multiplication was applied in both dimensions. The low-pass $J$-filter of the experiment was adjusted for an average coupling constant ${ }^{1} \mathrm{~J}(\mathrm{CH})$ of $147 \mathrm{~Hz}$ and the long-range delay utilized to excite the heteronuclear multiple quantum coherence was optimized for $7 \mathrm{~Hz}$.

Taking into account the nature of the phenolic compounds (phenylethanoids and flavones), their quantification was performed at $340 \mathrm{~nm}$ (Galvez, Martin-Cordero, Houghton, \& Ayuso, 2005) by the external standard method. The detection and quantification limits (LOD and $\mathrm{LOQ}$, respectively) were determined from the parameters of the calibration curves represented in Table 1, being defined as 3.3 and 10 times the value of the regression error divided by the slope, respectively (Ermer \& Miller, 2005; Snyder, Kirkland, \& Dolan, 2010).

Fractions 2 and 3 (verbascoside, isoverbascoside) were quantified using verbascoside as a reference compound. Apigenin-7-0-glucoside was used to quantify fractions 4 [isoscutellarein-7-0-allosyl $(1 \rightarrow 2$ ) 
Table 1

Linearity, LOD and LOQ of four standard compounds used as references.

\begin{tabular}{|c|c|c|c|c|c|c|c|}
\hline Standard compound & $\begin{array}{l}\text { Range concentration } \\
(\mu \mathrm{g} / \mathrm{mL})\end{array}$ & $\mathrm{n}^{\mathrm{a}}$ & $\begin{array}{l}\text { Slope }{ }^{\mathrm{b}} \\
\text { (area counts/mg) }\end{array}$ & $\begin{array}{l}\text { Intercept }{ }^{\mathrm{b}} \\
\text { (area counts/mg) }\end{array}$ & $\mathrm{R}^{2}$ & $\begin{array}{l}\text { LOD } \\
(\mu \mathrm{g} / \mathrm{mL})\end{array}$ & $\begin{array}{l}\text { LOQ } \\
(\mu \mathrm{g} / \mathrm{mL})\end{array}$ \\
\hline L-70-G & $45-473$ & 5 & $763( \pm 1) \times 10^{4}$ & $13( \pm 9) \times 10^{4}$ & 0.9967 & 32.5 & 98.4 \\
\hline Verb & $44-700$ & 5 & $166( \pm 6) \times 10^{4}$ & $6( \pm 2) \times 10^{3}$ & 0.9985 & 31.9 & 96.7 \\
\hline A-70-G & $40-500$ & 5 & $151( \pm 7) \times 10^{5}$ & $-6( \pm 1) \times 10^{5}$ & 0.9992 & 17.3 & 52.4 \\
\hline N-70-G & $5-68$ & 5 & $230( \pm 8) \times 10^{4}$ & $-2( \pm 6) \times 10^{3}$ & 0.9990 & 2.7 & 8.2 \\
\hline
\end{tabular}

L-70-G, luteolin-7-O-glucoside; Verb, verbascoside; A-7O-G, apigenin-7-O-glucoside; N-70-G, naringenin-7-0-glucoside.

a Number of points used for the regression of standard solutions. Injections were done in triplicate.

b The standard deviation in the slope and intercept of the regression line is shown in parentheses.

glucoside], 5 [isoscutellarein-7-0-(6-0-acetylallosyl) $(1 \rightarrow 6)$ glucoside], 7 [isoscutellarein-7-0-(6-O-acetylallosyl $)(1 \rightarrow 2)$ glucoside isomer], 9 [isoscutellarein-7-0-(6-O-acetylallosyl) $(1 \rightarrow 2)$ glucoside], 10 [4'-Omethylisoscutellarein-7-O-allosyl $(1 \rightarrow 2)$ glucoside], 11 [4'-O-methylisoscutellarein-7-O-(6-O-acetylallosyl) $(1 \rightarrow 2)$ glucoside], 8 (apigenin7-O-glucoside) and 12 (apigenin-7-O-rutinoside). Fraction 6 (luteolin7-O-glucoside) was quantified with luteolin-7-O-glucoside while naringenin-7-O-glucoside was used as the reference for quantification of phenolic compounds in fraction 13 (naringenin-7-0-rutinoside).

\section{Results and discussion}

The purified ethanolic extract of L. album (PEEL) represented $13 \%$ of the dried plant mass and its total phenolic compounds accounted for $192.5 \pm 10.3 \mathrm{mg} \mathrm{GAE} / \mathrm{g}$ of PEEL, which corresponds to a recovery of $24.24 \mathrm{mg} \mathrm{GAE} / \mathrm{g}$ of dried plant. This result is lower than that reported by Matkowski and Piotrowska (2006) (32.8 $\pm 4.0 \mathrm{mg} \mathrm{GAE} / \mathrm{g}$ of dried plant) and differences can be ascribed to various factors, such as different agronomic or extraction conditions.

\subsection{Identification of phenolic compounds in PEEL}

As can be observed in Fig. 1 and Table 2, the present study allowed identification of thirteen phenolic components in PEEL, which comprised flavones, phenylethanoid isomers and one flavanone. From the above compounds, derivatives of the uncommon flavone isoscutellarein were detected for the first time in the Lamium genus, and thus, their identification will be described below in detail.

\subsubsection{Isoscutellarein derivatives}

Overall, six isoscutellarein derivatives could be detected in PEEL (Table 2 and Fig. 2). These compounds, eluted in fractions 4, 5, 7, 9, 10 and 11 , showed characteristic UV spectra with maxima at 278 , 302 and $333 \mathrm{~nm}$, which is in agreement with that described for isoscutellarein glucosides (Innocenti et al., 2007; Sahin, Ezer, \& Calis, 2006; Saracoglu, Harput, \& Ogihara, 2004). Notably, this is the first study reporting this flavonoid aglycone class in the Lamium genus, refuting previous chemotaxonomic studies of the plant (TomásBarberán, Grayer-Barkmeijer, Gil, \& Harborne, 1988).

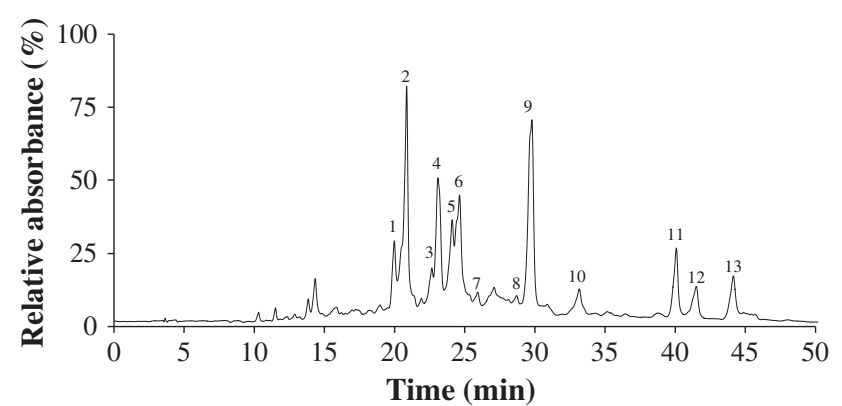

Fig. 1. Chromatographic profile at $340 \mathrm{~nm}$ of purified ethanolic extract of Lamium album $\mathrm{L}$.
In more detail, the phenolic compound of fraction 4 corresponded to isoscutellarein-7-0-allosyl $(1 \rightarrow 2)$ glucoside. This compound was detected in the ESI-MS spectrum as a $[\mathrm{M}-\mathrm{H}]^{-}$ion at $m / z 609$, and its main product ion $(\mathrm{m} / \mathrm{z} 285)$ was formed by the loss of $324 \mathrm{Da}$, which indicates an $\mathrm{O}$-glycosylation on a phenolic hydroxyl with a dihexoside (Ferreres, Llorach, \& Gil-Izquierdo, 2004). Moreover, the product ion $[\mathrm{M}-\mathrm{H}-180]^{-}$at $\mathrm{m} / \mathrm{z} 429$ indicated the $1 \rightarrow 2$ glycosylation between the sugars (Ferreres et al., 2004; Petreska et al., 2011). Note that this compound has been previously described to occur in genus Stachys and Sideritis, both belonging to the same subfamily (Lamioideae) as Lamium (Ferreres et al., 2004; Petreska et al., 2011; Tomás-Barberán, Francisco, Gil, Ferreres, \& Tomás-Lorente, 1992).

In a similar way, the compound eluting in fraction $10\left([\mathrm{M}-\mathrm{H}]^{-}\right.$ion at $m / z 623$ ) was tentatively assigned as the $4^{\prime}-0$-methyl derivative of the previous compound. Besides the characteristic base peak in $\mathrm{MS}^{2}$ spectrum at $m / z 299(-324 \mathrm{Da})$ and the product ions [M-H-162 $]^{-}$ (ion at $m / z 461$ ) and $[\mathrm{M}-\mathrm{H}-180]^{-}$(ion at $m / z 443$ ), due to loss of the hexose as residue and as unit, respectively, this compound also showed the simultaneous loss of the disaccharide moiety and a methyl group (ion at $m / z 284$ ), which is in agreement with the pattern fragmentation of $4^{\prime}$-O-methylisoscutellarein-7-O-allosyl $(1 \rightarrow 2)$ glucoside, recently detected in Stachys and Sideritis genus (Karioti, Bolognesi, Vincieri, \& Bilia, 2010; Petreska et al., 2011)

Isoscutellarein acetyl derivatives were also found in PEEL (fractions $5,7,9$ and 11), as confirmed by the initial loss of $42 \mathrm{Da}$ in their MS ${ }^{2}$ spectra. From those, the isomeric compounds (MW $652 \mathrm{Da}$ ) which eluted in the first three fractions were the acetyl derivatives of isoscutellarein-O-allosyl $(1 \rightarrow 2$ )glucoside (compound of fraction 4 ) and of $4^{\prime}$-O-methylisoscutellarein-7-O-allosyl $(1 \rightarrow 2)$ glucoside (compound of fraction 10).

The $\mathrm{MS}^{2}$ spectrum of the major acetylated isomer of isoscutellarein0 -allosyl $(1 \rightarrow 2)$ glucoside, eluted in fraction $9\left([\mathrm{M}-\mathrm{H}]^{-}\right.$ion at $\left.m / z 651\right)$, showed a base peak at $m / z 285\left([\mathrm{M}-\mathrm{H}-324-42]^{-}\right)$, which is indicative for $\mathrm{O}$-acetylglycosylation onto the phenolic hydroxyl groups (Petreska et al., 2011). Moreover, the intermediate ion $[\mathrm{M}-\mathrm{H}-42-180]^{-}$at $\mathrm{m} / \mathrm{z}$ 429 was indicative of an acetyl group on the external sugar (Karioti et al., 2010). Overall, the fragmentation pattern of this compound corresponded to that of isoscutellarein-7-0-(6-0-acetylallosyl $)(1 \rightarrow 2)$ glucoside. Moreover, this assignment was confirmed by NMR spectroscopy, as all the ${ }^{1} \mathrm{H}$ NMR and ${ }^{13} \mathrm{C}$ NMR signals (Table 3 ) were consistent with that isoscutellarein derivative (Albach, Grayer, Jensen, Ozgokce, \& Veitch, 2003; Gabrieli, Kefalas, \& Kokkalou, 2005; Sahin et al., 2006).

Regarding the remaining isoscutellarein acetyl derivatives (fractions 5 and 7), they should have distinct 0 -acylation and/or glycosylation with respect to the previous compound. At this point, the exact features of those groups could not be determined. Even so, it is possible to predict that the isomer in fraction 7 also contains a $1 \rightarrow 2$ glycosylation, as dictated by the occurrence of the product ion at $m / z 429$ ([M-H$\left.180-42]^{-}\right)$in its $\mathrm{MS}^{2}$ spectrum. This isomer must correspond to isoscutellarein 7-O-(4-O-acetylallosyl $)(1 \rightarrow 2)$ glucoside or to isoscutellarein 7-O-(2-O-acetylallosyl) $(1 \rightarrow 2)$ glucoside, since acylations of flavonoid glycosides can also occur in 2- and 4-positions of the hexose (Cuyckens \& Claeys, 2004). On the other hand, the 0 -glycosylation type of the 
Table 2

Identification of HPLC eluting fractions by HPLC-DAD, ESI-MS and ESI-MS ${ }^{\mathrm{n}}$ from ethanolic extract of Lamium album L.

\begin{tabular}{|c|c|c|c|c|c|}
\hline Peak & $\begin{array}{l}\mathrm{RT} \\
(\min )\end{array}$ & $\lambda_{\max }$ & {$[\mathrm{M}-\mathrm{H}]^{-}$} & $\begin{array}{l}\text { Main fragment } \\
\text { ESI-MS }^{\mathrm{n}}\end{array}$ & Compound \\
\hline 1 & 20.0 & $\begin{array}{l}254,267 \\
345\end{array}$ & - & ( & Luteolin derivative \\
\hline 2 & 20.9 & 290,329 & 623 & $\begin{array}{l}\operatorname{MS}^{2} \text { [623]: } 477 \text { (2\%), 461; } \text { MS }^{3} \text { [461]: } 315 \text { (100\%), } 297 \text { (10\%), } \\
135 \text { (30\%) }\end{array}$ & Verbascoside \\
\hline 3 & 22.7 & 290,328 & 623 & $\begin{array}{l}\text { MS }^{2} \text { [623]: } 477 \text { (2\%), } 461 \text { (100\%), } 299 \text { (5\%); } \text { MS }^{3} \text { [461]: } 315 \text { (100\%), } \\
297 \text { (10\%), } 161 \text { (3\%), } 135 \text { (30\%); } \text { MS }^{4}[315]: 135\end{array}$ & Isoverbascoside \\
\hline 4 & 23.1 & $\begin{array}{l}275,302, \\
333\end{array}$ & 609 & $\begin{array}{l}\text { MS }^{2} \text { [609]: } 489 \text { (2\%), } 447 \text { (20\%), } 429(40 \%) 285(100 \%) \\
\text { MS }^{3} \text { [429]: } 285 \text { (100\%), } 284(10 \%) ; M^{4}[285]: 267(5 \%) \\
257 \text { (20\%), } 241(100 \%), 213(40 \%), 199(3 \%), 197(4 \%) \\
191(10 \%) ; \text { MS }^{5}[241]: 213(100 \%), 197(40 \%), 185(45 \%) \\
145 \text { (10\%) }\end{array}$ & Isoscutellarein-7-O-allosyl $(1 \rightarrow 2)$ glucoside \\
\hline 5 & 24.1 & $\begin{array}{l}275,302, \\
333\end{array}$ & 651 & $\begin{array}{l}\text { MS }^{2} \text { [651]: } 609 \text { (100\%), } 285(2 \%) ; \mathrm{MS}^{3}[609]: 489(4 \%) 447(85 \%), \\
285 \text { (100\%); } \mathrm{MS}^{4}[447]: 285 ; \mathrm{MS}^{5}[285]: 267(3 \%), 243(60 \%), \\
241(100 \%), 217(35 \%), 199(43 \%), 175(40 \%), 151(3 \%)\end{array}$ & Isoscutellarein-7-O-(6-O-acetylallosyl $)(1 \rightarrow 6)$ glucoside \\
\hline 6 & 24.6 & $\begin{array}{l}254,267 \\
345\end{array}$ & 447 & $\begin{array}{l}\text { MS }^{2} \text { [447]: } 285 ; \mathrm{MS}^{3} \text { [285]: } 243 \text { (5\%), } 241 \text { (100\%), } 217 \text { (60\%), } \\
199 \text { (60\%), } 175(60 \%)\end{array}$ & Luteolin-7-O-glucoside \\
\hline 7 & 25.9 & $\begin{array}{l}275,302, \\
333\end{array}$ & 651 & $\begin{array}{l}\operatorname{MS}^{2} \text { [651]: } 609 \text { (100\%), } 591 \text { (10\%), } 447 \text { (2\%), } 429 \text { (5\%), } 285 \text { (20\%); } \\
\text { MS }^{3} \text { [609]: } 447 \text { (5\%), } 429 \text { (30\%), } 285(100 \%)\end{array}$ & $\begin{array}{l}\text { Isoscutellarein-7-O-(6-O-acetylallosyl })(1 \rightarrow 2) \text { glucoside } \\
\text { isomer }\end{array}$ \\
\hline 8 & 28.7 & 266,342 & 431 & $\begin{array}{l}\operatorname{MS}^{2} \text { [431]: 269; } \mathrm{MS}^{3} \text { [269]: } 227 \text { (100\%), } 225 \text { (90\%), } 199 \text { (85\%), } \\
180 \text { (95\%) }\end{array}$ & Apigenin-7-O-glucoside \\
\hline 9 & 29.8 & $\begin{array}{l}275,302, \\
333\end{array}$ & 651 & $\begin{array}{l}\operatorname{MS}^{2}[651]: 609(15 \%), 591(10 \%), 447(7 \%), 429(45 \%), 285(100 \%) ; \\
\text { MS }^{3} \text { [429]: } 285 ; \text { MS }^{4}[285]: 257(30 \%), 241(100 \%), 213(30 \%), 191(7 \%), \\
171 \text { (4\%) }\end{array}$ & Isoscutellarein-7-O-(6-O-acetylallosyl) $(1 \rightarrow 2)$ glucoside \\
\hline 10 & 33.2 & $\begin{array}{l}275,305 \\
327\end{array}$ & 623 & $\begin{array}{l}\text { MS }^{2} \text { [623]: } 461 \text { (15\%), } 443 \text { (3\%), } 299 \text { (100\%), } 284 \text { (10\%); } \text { MS }^{3} \text { [461]: 299; } \\
\text { MS }^{4} \text { [299]: } 284 \text { (100\%), } 255 \text { (1\%), } 240(4 \%)\end{array}$ & 4'-O-Methylisoscutellarein-7-O-allosyl $(1 \rightarrow 2)$ glucoside \\
\hline 11 & 40.1 & $\begin{array}{l}275,305 \\
327\end{array}$ & 665 & $\begin{array}{l}\operatorname{MS}^{2} \text { [665]: } 623 \text { (15\%), } 461(10 \%), 443(5 \%), 299(100 \%), 284(15 \%) ; \\
\text { MS }^{3} \text { [461]: } 299 ; \mathrm{MS}^{4} \text { [299]: } 284(100 \%), 255(1 \%), 256(1 \%), 240(5 \%) \\
227 \text { (1\%); } \mathrm{MS}^{5}[284]: 283(100 \%), 256(25 \%), 227(20 \%), 228(19 \%) \\
212(8 \%), 200(4 \%), 150(1 \%), 137(7 \%)\end{array}$ & $\begin{array}{l}\text { 4'-O-Methylisoscutellarein-7-O-(6-O-acetylallosyl })(1 \rightarrow 2) \\
\text { glucoside }\end{array}$ \\
\hline 12 & 41.5 & 266,342 & 577 & $\begin{array}{l}\text { MS }^{2} \text { [577]: } 431 \text { (1\%), } 307 \text { (3\%), } 269 \text { (100\%); } \text { MS }^{3} \text { [269]: } 227 \text { (10\%), } \\
225 \text { (100\%), } 201 \text { (15\%), } 183 \text { (2\%), } 151(10 \%), 149(15 \%)\end{array}$ & Apigenin-7-O-rutinoside \\
\hline 13 & 44.2 & - & 579 & $\begin{array}{l}\mathrm{MS}^{2} \text { [579]: } 307 \text { (75\%), } 271(100 \%) ; \mathrm{MS}^{3}[307]: 247 \text { (25\%), } 205(20 \%), \\
187 \text { (25\%), } 175 \text { (3\%), } 163 \text { (50\%), } 145 \text { (100\%); } \mathrm{MS}^{3}[271]: 177(10 \%), \\
151 \text { (100\%) }\end{array}$ & Naringenin-7-O-rutinoside \\
\hline
\end{tabular}

Peak 1 assignment was only based on UV spectra, which corresponded to that of luteolin.

isomer eluted in fraction 5 differs from that of the other two. Probably this is a $1 \rightarrow 6$ glycosidic type ligation, since the product ion $[\mathrm{M}-\mathrm{H}-42-162]^{-}$(at $\mathrm{m} / \mathrm{z} 447$ ) was prevalent while $[\mathrm{M}-\mathrm{H}-42-$ $180]^{-}$or $[\mathrm{M}-\mathrm{H}-180]^{-}$product ion was not observed in $\mathrm{MS}^{\mathrm{n}}$ experiments (Ferreres et al., 2004). To our knowledge, isoscutellarein-7-O$(6-0$-acetylallosyl $)(1 \rightarrow 2)$ glucoside isomers with distinct 0 -acylation and/or glycosylation positions have not been described in literature so far.

The acetylated form of 4'-O-methylisoscutellarein-7-O-allosyl $(1 \rightarrow 2)$ glucoside (MW $666 \mathrm{Da}$ ) was found in fraction 11. Accordingly, the MS spectrum of this fraction showed the $[\mathrm{M}-\mathrm{H}]^{-}$at $m / z 665$ and its $\mathrm{MS}^{2}$ spectrum showed high relative abundance ions at $\mathrm{m} / \mathrm{z} 299$ and at $\mathrm{m} / \mathrm{z}$ $623\left([\mathrm{M}-\mathrm{H}-42]^{-}\right)$(correspondent to methylisoscutellarein). Moreover, the fragmentation pattern of the latter ion was similar to that described for the 4'-O-methylisoscutellarein-7-O-allosyl $(1 \rightarrow 2)$ glucoside (fraction 10).

\subsubsection{Other phenolic compounds}

Besides the isoscutellarein derivatives previously described, PEEL also contained glycosides of common flavones, namely luteolin-7-Oglucoside (fraction 6), apigenin-7-0-glucoside (fraction 8), apigenin7-0-rutinoside (fraction 12), the flavanone naringenin-7-0-rutinoside (fraction 13) and two phenylethanoid glycosides (verbascoside and isoverbascoside, in fractions 2 and 3, respectively). The latter showed UV data and fragmentation pathway similar to that described in literature (Li, Liu, Liu, Tsao, \& Liu, 2009). In particular, the MS ${ }^{2}$ of their molecular ion $\left([\mathrm{M}-\mathrm{H}]^{-}\right.$at $\left.m / z 623\right)$ showed a base peak product ion resultant from the loss of caffeoyl $\left(-162 \mathrm{Da}\right.$, ion at $\mathrm{m} / z$ 461) while the MS ${ }^{3}$ data of this latter ion supported the main loss of a rhamnose unit (ion at $m / z 315$ ). Note that the phenylethanoid glycoside eluting in the most intense HPLC peak (fraction 2) corresponded to verbascoside, which has previously been described to occur in several Lamium species, including in L. album (Budzianowski \& Skrzypczak, 1995). Still, to

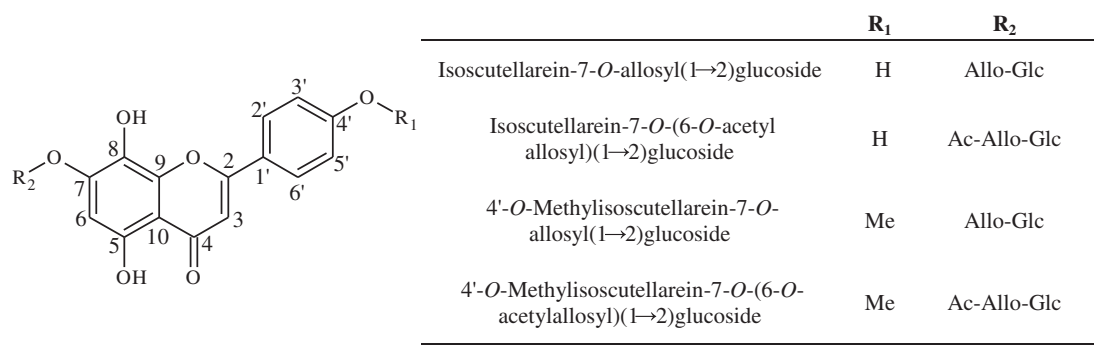

Allo, Allosyl unit; Glc, Glucosyl unit; Ac, Acetyl unit; Me, Methyl unit

Fig. 2. Main features of isoscutellarein derivatives found in purified extract of Lamium album L. 
Table 3

${ }^{13} \mathrm{C}$ and ${ }^{1} \mathrm{H}$ NMR spectral data for the compound isoscutellarein-7-O-(6-O-acetylallosyl) $\left(1 \rightarrow 2\right.$ )glucoside obtained from purified phenolic extract of Lamium album L. (in DMSO- $\mathrm{d}_{6}$ ).

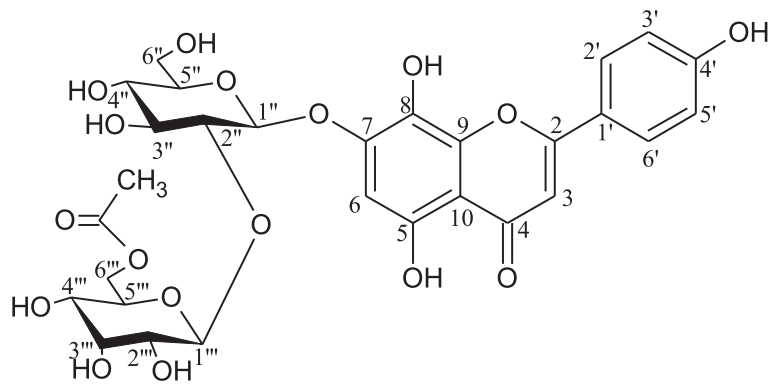

\begin{tabular}{|c|c|c|c|c|c|}
\hline Atom & ${ }^{13} \mathrm{C}$ & ${ }^{1} \mathrm{H}$ & Atom & ${ }^{13} \mathrm{C}$ & ${ }^{1} \mathrm{H}$ \\
\hline \multicolumn{3}{|c|}{ Aglycone } & \multicolumn{3}{|c|}{ Glucose $^{\mathrm{a}}$} \\
\hline 2 & 164.1 & - & $1 "$ & 100.0 & $5.09(\mathrm{~d}, J=7.4 \mathrm{~Hz})$ \\
\hline 3 & 102.6 & 6.85 & $2 "$ & 82.6 & $3.59(' \mathrm{t}$ ', $J=8.3 \mathrm{~Hz})$ \\
\hline 4 & 182.4 & - & 3" & 75.6 & - \\
\hline 5 & 152.2 & 12.38 & 4" & 69.2 & - \\
\hline 6 & 100.0 & 6.70 & $5 "$ & 77.1 & - \\
\hline 7 & 150.5 & - & 6" & 60.5 & $3.74(\mathrm{dd}, J=10.6$ and $5.3 \mathrm{~Hz})$ \\
\hline 8 & 127.5 & 7.95 & \multicolumn{3}{|r|}{ (1) } \\
\hline 9 & 143.7 & - & 1"' & 102.6 & $4.92(\mathrm{~d}, J=7.9 \mathrm{~Hz})$ \\
\hline 10 & 105.5 & - & 2"' & 71.4 & - \\
\hline $1^{\prime}$ & 121.2 & - & 3"' & 70.8 & $3.92-3.90$ \\
\hline $2^{\prime}$ & 128.7 & $8.00(\mathrm{~d}, J=8.5 \mathrm{~Hz})$ & 4"' & 66.8 & $3.92-3.90$ \\
\hline $3^{\prime}$ & 115.9 & $6.95(\mathrm{~d}, J=8.5 \mathrm{~Hz})$ & 5"' & 71.5 & $3.88-3.86$ \\
\hline $4^{\prime}$ & 161.3 & - & 6"' & 63.5 & $4.02(\mathrm{~d}, J=2.7 \mathrm{~Hz})$ \\
\hline $5^{\prime}$ & 115.9 & $6.95(\mathrm{~d}, J=8.5 \mathrm{~Hz})$ & $\mathrm{OAC}$ & & \\
\hline \multirow[t]{2}{*}{$6^{\prime}$} & 128.7 & $8.00(\mathrm{~d}, J=8.5 \mathrm{~Hz})$ & & 20.5 & 1.88 \\
\hline & & & & 170.3 & - \\
\hline
\end{tabular}

${ }^{a}$ The $\mathrm{OH}$ groups of the sugar moiety appear at: $5.25(\mathrm{~d}, J=5.5 \mathrm{~Hz}, 1 \mathrm{H}), 5.16$ $(\mathrm{d}, J=4.2 \mathrm{~Hz}, 1 \mathrm{H}),, 5.02(\mathrm{~d}, J=3.4 \mathrm{~Hz}, 1 \mathrm{H}), 4.83(\mathrm{~d}, J=7.8 \mathrm{~Hz}, 1 \mathrm{H}), 4.74(\mathrm{t}, J=5.5 \mathrm{~Hz}, 1 \mathrm{H})$.

our knowledge, isoverbascoside (fraction 3 ) is herein described for the first time in this species.

\subsection{Quantification of phenolic compounds in PEEL}

The quantification of the distinct phenolic compounds in PEEL extract was carried out using calibration curves of each available standard. Table 1 shows typical analytical parameters including the limits of detection and quantification (LOD and LOQ, respectively), the calibration curves, the linearity and the regression coefficient $\left(\mathrm{R}^{2}\right)$.

The quantified phenolic compounds in the ethanolic extract of L. album accounted for $500.7 \pm 50.0 \mathrm{mg} / \mathrm{g}$ of extract (Table 4 ), that is equivalent to $14.9 \mathrm{mg} / \mathrm{g}$ of dry plant. This extract was mainly rich in verbascoside, which, together with isoverbascoside, accounted for approximately $55 \%$ of the total phenolic content of PEEL. Also important, the glucosyl-isoscutellarein derivatives of this extract were present in appreciable amounts (total of $27 \%$ ), mostly in the acetylated form (18\%). Still note that accurate quantification of these compounds can be impaired, as optimum peak separation was not achieved for all the compounds and apigenin-7-O-glucoside was used as a reference for isoscutellarein derivative quantification, instead of the exact reference compounds.

The high content of the phenylethanoid glycosides verbascoside and isoverbascoside in the ethanolic extract of $L$. album suggests that medicinal activities claimed to this plant can be associated with these compounds. In fact, several studies reported important activities for verbascoside, including antioxidant and free radical scavenging capacity, neuroprotective, hepatoprotective, analgesic, cytotoxic, antimicrobial, anti-inflammatory and beneficial effects on the cardiovascular system. Most of these activities are also ascribed to isoverbascoside (Fu, Pang, \& Wong, 2008; Isacchi et al., 2011; Korkina, 2007; Kostyuk, Potapovich, Suhan, de Luca, \& Korkina, 2011; Morikawa et al., 2010). Moreover, it is important to highlight that despite the presence of lower amounts of isoscutellarein derivatives as compared to those of phenylethanoid glycosides, these can also be key components on the ethnopharmacological effects of the plant. Indeed, for the last decades, isoscutellarein derivatives have also been described to exert important beneficial activities as antiviral, antioxidant, cytotoxic, antinociceptive, anti-inflammatory and inhibitory activity against osteoclastogenesis (Kupeli, Sahin, Yesilada, Calis, \& Ezer, 2007; Nagai, Miyaichi, Tomimori, Suzuki, \& Yamada, 1992; Yang et al., 2003; Yoon, Jeong, Hwang, Ryu, \& Kim, 2007).

\section{Conclusions}

The phenolic composition of the purified ethanolic extract of aerial parts of L. album was assessed by a combined method using HPLC-DAD and ESI-MS. The extract was mainly rich in the two phenylethanoids verbascoside and isoverbascoside (55\%), where the accounted amount of the former was 6 fold of that of the latter. Other important phenolic portions of the extract $(27 \%)$ were derived from the unusual flavone isoscutellarein. Thus, the compounds isoscutellarein-7-0allosyl $(1 \rightarrow 2)$ glucoside, isoscutellarein-7-0-(6-0-acetylallosyl) $(1 \rightarrow 6)$ glucoside, isoscutellarein-7-O-(6-0-acetylallosyl) $(1 \rightarrow 2)$ glucoside and its structural isomer, $4^{\prime}$-O-methylisoscutellarein-7-O-allosyl $(1 \rightarrow 2)$ glucoside and 4'-O-methylisoscutellarein-7-O-(6-0-acetylallosyl) $(1 \rightarrow 2)$ glucoside were herein described for the first time in the genus Lamium. Apigenin-7-O-glucoside, luteolin-7-O-glucoside, apigenin-7-O-rutinoside and the flavanone naringenin-7-O-rutinoside were minor constituents of this extract. Thus, overall, this work is an important contribution to

Table 4

Quantification of the identified compounds in ethanolic extract of Lamium album L.

\begin{tabular}{|c|c|c|c|}
\hline Peak & Compound & Quantified with & $\mathrm{mg} / \mathrm{g}$ extract \\
\hline 2 & Verbascoside & Verbascoside & $233.7 \pm 13.6$ \\
\hline 3 & Isoverbascoside & Verbascoside & $39.2 \pm 5.6$ \\
\hline 4 & Isoscutellarein-7-O-allosyl $(1 \rightarrow 2)$ glucoside & Apigenin-7-O-glucoside & $26.8 \pm 5.3$ \\
\hline 5 & Isoscutellarein-7-O-(6-O-acetylallosyl $)(1 \rightarrow 6)$ glucoside & Apigenin-7-O-glucoside & $23.6 \pm 6.7$ \\
\hline 6 & Luteolin-7-O-glucoside & Luteolin-7-O-glucoside & $29.7 \pm 2.2$ \\
\hline 7 & Isoscutellarein-7-O-(6-O-acetylallosyl $)(1 \rightarrow 2)$ glucoside isomer & Apigenin-7-O-glucoside & $9.6 \pm 0.3$ \\
\hline 8 & Apigenin-7-O-glucoside & Apigenin-7-O-glucoside & $16.1 \pm 5.8$ \\
\hline 9 & Isoscutellarein-7-O-(6-O-acetylallosyl $)(1 \rightarrow 2)$ glucoside & Apigenin-7-O-glucoside & $37.4 \pm 4.4$ \\
\hline 10 & 4'-O-Methylisoscutellarein-7-O-allosyl $(1 \rightarrow 2)$ glucoside & Apigenin-7-O-glucoside & $16.6 \pm 6.5$ \\
\hline 11 & 4'-O-Methylisoscutellarein-7-O-(6-O-acetylallosyl $)(1 \rightarrow 2)$ glucoside & Apigenin-7-O-glucoside & $19.4 \pm 5.2$ \\
\hline 12 & Apigenin-7-O-rutinoside & Apigenin-7-O-glucoside & $16.2 \pm 4.7$ \\
\hline 13 & Naringenin-7-O-rutinoside & Naringenin-7-O-glucoside & $32.6 \pm 5.6$ \\
\hline
\end{tabular}

Mean values \pm standard deviations. 
the chemical characterization of the L. album emphasizing that its main phenolic constituents are important antioxidant agents (verbascoside, isoverbascoside and isoscutellarein derivatives) which have been associated with diverse beneficial effects on human health. Further work is now being undertaken by our group in order to evaluate the relation of these phenolic constituents with the antioxidant capacity of L. album. We expect that if positive relations are established, consumers' and the food industry's interest in this plant will be raised.

\section{Acknowledgments}

The authors acknowledge the financial support provided by the FCT to CERNAS (project PEst-OE/AGR/UI0681/2011) and of the FCT as well as FSE (III Quadro Comunitário de Apoio) to QOPNA (project PEst-C/QUI/UI0062/2011), REDE/1504/REM/2005 (that concerns the Portuguese Mass Spectrometry Network) and the Portuguese National NMR Network (RNRMN). Olívia R Pereira was supported by a PhD grant (SFRH/PROTEC/49600/2009).

\section{References}

Albach, D. C., Grayer, R. J., Jensen, S. R., Ozgokce, F., \& Veitch, N. C. (2003). Acylated flavone glycosides from Veronica. Phytochemistry, 64(7), 1295-1301.

Baranov, A. I. (1967). Wild vegetables in Manchuria. Economic Botany, 21, 140-155.

Budzianowski, J., \& Skrzypczak, L. (1995). Phenylpropanoid esters from Lamium album flowers. Phytochemistry, 38(4), 997-1001.

Celnet (2005). Celnet recipes: Wild food guide for white deadnettle (Lamium album) Retrieved from http://www.celtnet.org.uk/recipes/

Clifford, A. W. (2001). Mediterranean vegetables: A cook's ABC of vegetables and their preparation. Boston, Massachusetts: The Harvard Common Press.

Cuyckens, H., \& Claeys, M. (2004). Mass spectrometry in the structural analysis of flavonoids. Journal of Mass Spectrometry, 39(4), 1-15.

Denisow, B., \& Bozek, M. (2008). Blooming and pollen production of two Lamium L. species. Journal of Apicultural Science, 52(1), 21-30.

Ermer, J., \& Miller, J. M. (2005). Method validation in pharmaceutical analysis: A guide to best practice. Weinheim: WILEY-VCH Verlag GmbH \& Co.

Ferreres, F., Llorach, R., \& Gil-Izquierdo, A. (2004). Characterization of the interglycosidic linkage in di-, tri-, tetra- and pentaglycosylated flavonoids and differentiation of positional isomers by liquid chromatography/electrospray ionization tandem mass spectrometry. Journal of Mass Spectrometry, 39(3), 312-321.

Fu, G., Pang, H., \& Wong, Y. H. (2008). Naturally occurring phenylethanoid glycosides: Potential leads for new therapeutics. Current Medicinal Chemistry, 15(25), 2592-2613.

Gabrieli, C. N., Kefalas, P. G., \& Kokkalou, E. L. (2005). Antioxidant activity of flavonoids from Sideritis raeseri. Journal of Ethnopharmacology, 96(3), 423-428.

Galvez, M., Martin-Cordero, C., Houghton, P. J., \& Ayuso, M. J. (2005). Antioxidant activity of Plantago bellardii All. Phytotherapy Research, 19(12), 1074-1076.

Harford, R. (2007). Eat weeds: Wild food guide to the edible plants of Britain. Retrieved from http://www.eatweeds.co.uk/

Heinrich, M., Müller, W. E., \& Galli, C. (2006). Local Mediterranean food plants and nutraceuticals, Vol. 59, Forum Nutr. Basel, Karger.

Innocenti, M., Michelozzi, M., Giaccherini, C., Ieri, F., Vincieri, F. F., \& Mulinacci, N. (2007). Flavonoids and biflavonoids in Tuscan berries of Juniperus communis L.: Detection and quantitation by HPLC/DAD/ESI/MS. Journal of Agricultural and Food Chemistry, 55(16), 6596-6602.

Isacchi, B., Iacopi, R., Bergonzi, M. C., Ghelardini, C., Galeotti, N., Norcini, M., et al. (2011). Antihyperalgesic activity of verbascoside in two models of neuropathic pain. Journal of Pharmacy and Pharmacology, 63(4), 594-601.

Karioti, A., Bolognesi, L., Vincieri, F. F., \& Bilia, A. R. (2010). Analysis of the constituents of aqueous preparations of Stachys recta by HPLC-DAD and HPLC-ESI-MS. Journal of Pharmaceutical and Biomedical Analysis, 53(1), 15-23.

Korkina, L. G. (2007). Phenylpropanoids as naturally occurring antioxidants: From plant defense to human health. Cellular and Molecular Biology, 53(1), 15-25.

Kostyuk, V. A., Potapovich, A. I., Suhan, T. O., de Luca, C., \& Korkina, L. G. (2011). Antioxidant and signal modulation properties of plant polyphenols in controlling vascular inflammation. European Journal of Pharmacology, 658(2-3), 248-256.
Kupeli, E., Sahin, F. P., Yesilada, E., Calis, I., \& Ezer, N. (2007). In vivo anti-inflammatory and antinociceptive activity evaluation of phenolic compounds from Sideritis stricta. Zeitschrift fur Naturforschung. C, Journal of Biosciences, 62(7-8), 519-525.

Li, L., Liu, C. M., Liu, Z. Q., Tsao, R., \& Liu, S. Y. (2009). Identification of phenylethanoid glycosides in plant extract of Plantago asiatica L. by liquid chromatographyelectrospray ionization mass spectrometry. Chinese Journal of Chemistry, 27(3), 541-545.

Luczaj, L. (2008). Archival data on wild food plants used in Poland in 1948. Journal of Ethnobiology and Ethnomedicine, 4, 4.

Matkowski, A., \& Piotrowska, M. (2006). Antioxidant and free radical scavenging activities of some medicinal plants from the Lamiaceae. Fitoterapia, 77(5), 346-353.

Mihaly Cozmuta, A., Bretan, L., Mihaly Cozmuta, L., Nicula, C., \& Peter, A. (in press). Lead traceability along soil-melliferous flora-bee family-apiary products chain. Journal of Environmental Monitoring, doi:10.1039/C2EM30084B.

Morikawa, T., Pan, Y., Ninomiya, K., Imura, K., Matsuda, H., Yoshikawa, M., et al. (2010). Acylated phenylethanoid oligoglycosides with hepatoprotective activity from the desert plant Cistanche tubulosa. Bioorganic \& Medicinal Chemistry, 18(5), 1882-1890.

Nagai, T., Miyaichi, Y., Tomimori, T., Suzuki, Y., \& Yamada, H. (1992). In vivo antiinfluenza virus activity of plant flavonoids possessing inhibitory activity for influenza virus sialidase. Antiviral Research, 19(3), 207-217.

Ninomiya, K., Nishida, S., Matsura, Y., Asada, M., Kawahara, Y., Yoshikawa, M., et al. (2006). Fat-metabolism improving agent for use in food/drink for improving fat metabolism and preventing/treating lifestyle related disease e.g. diabetes, contains polar solvent extract of herb e.g. rose hip fruit, mugwort or safflower. In MORISHITA JINTAN KK, (MORI-Non-standard) (pp. 19).

Paduch, R., Matysik, G., Wojciak-Kosior, M., Kandefer-Szerszen, M., Skalska-Kaminska, A., Nowak-Kryska, M., et al. (2008). Lamium album extracts express free radical scavenging and cytotoxic activities. Polish Journal of Environmental Studies, 17(4), 569-580.

Paduch, R., Wójciak-Kosior, M., \& Matysik, G. (2007). Investigation of biological activity of Lamii albi flos extracts. Journal of Ethnopharmacology, 110(1), 69-75.

Pereira, O. R., Silva, A. M. S., Domingues, M. R. M., \& Cardoso, S. M. (2012). Identification of phenolic constituents of Cytisus multiflorus. Food Chemistry, 131(2), 652-659.

Petreska, J., Stefova, M., Ferreres, F., Moreno, D. A., Tomás-Barberán, F. A., Stefkov, G., et al. (2011). Potential bioactive phenolics of Macedonian Sideritis species used for medicinal "Mountain Tea". Food Chemistry, 125(1), 13-20.

Sahin, F. P., Ezer, N., \& Calis, I. (2006). Terpenic and phenolic compounds from Sideritis stricta. Turkish Journal of Chemistry, 30(4), 495-504.

Saracoglu, I., Harput, U. S., \& Ogihara, Y. (2004). Acylated flavone glycosides from Veronica pectinata var. glandulosa and V-persica. Turkish Journal of Chemistry, 28(6), 751-759.

Singleton, V. L., \& Rossi, J. A. (1965). Colorimetry of total phenolics with phosphomolybdicphosphotungstic acid reagents. American Journal of Enology and Viticulture, 16, 144-158.

Snyder, L., Kirkland, J., \& Dolan, J. (2010). Introduction to modern liquid chromatography. New Jersey: John Wiley \& Sons, Inc..

Sturtevants, E. L. (1919). Edible plants of the world. (Vol.). : The Southwest School of Botanical Medicine.

Tomás-Barberán, F. A., Francisco, A., Gil, M. I., Ferreres, F., \& Tomás-Lorente, F. (1992). Flavonoid p-coumaroylglucosides and 8-hydroxyflavone allosylglucosides in some labiatae. Phytochemistry, 31(9), 3097-3102.

Tomás-Barberán, F. A., Grayer-Barkmeijer, R. J., Gil, M. I., \& Harborne, J. B. (1988). Distribution of 6-hydroxy-, 6-methoxy- and 8-hydroxyflavone glycosides in the labiatae, the scrophulariaceae and related families. Phytochemistry, 27(8), 2631-2645.

Turner, N. J., Luczaj, L. J., Migliorini, P., Pieroni, A., Dreon, A. L., Sacchetti, L. E., et al. (2011). Edible and tended wild plants, traditional ecological knowledge and agroecology. Critical Reviews in Plant Sciences, 30(1-2), 198-225.

Valyova, M. S., Dimitrova, M. A., Ganeva, Y. A., Mihova Kapchina-Toteva, V., \& Petkova Yordanova, Z. (2011). Evaluation of antioxidant and free radical scavenging potential of Lamium album L. growing in Bulgaria. Journal of Pharmacy Research, 4(4), 945-947.

$\mathrm{Xu}, \mathrm{F}$. (2008). Chinese medicine e.g. for treating arthropathy, comprises broad cocklebur, vervain, condyle grass, motherwort, saxifrage, cactus, mulberry branch, white dead nettle, Boston ivy, Folium photiniae, water pepper and Chinese fever vine. In XU F, (XUFF-Individual) (pp. 10).

Yalcin, F. N., \& Kaya, D. (2006). Ethnobotany, pharmacology and phytochemistry of the Genus Lamium (Lamiaceae). FABAD Journal of Pharmaceutical Sciences, 31, 43-52.

Yang, H., Protiva, P., Cui, B. L., Ma, C. Y., Baggett, S., Hequet, V., et al. (2003). New bioactive polyphenols from Theobroma grandiflorum ("Cupuacu”). Journal of Natural Products, 66(11), 1501-1504

Yoon, K. D., Jeong, D. G., Hwang, Y. H., Ryu, J. M., \& Kim, J. (2007). Inhibitors of osteoclast differentiation from Cephalotaxus koreana. Journal of Natural Products, 70(12), 2029-2032. 


\subsection{Phenolic characterization of Leonurus cardiaca L. EXTRACTS}

Leonurus cardiaca L. (motherwort), subfamily Lamioideae (Lamiaceae) is a plant native to central Europe but spread to countries of diverse climate around the world. It easily grows in different types of soil and is commonly found in pastures, road edges, abandoned parks, waste ground, i.e. globally found in rough locations [1, 2]. In traditional medicine, infusions of aerial parts of motherwort are used due to its beneficial effects in climacteric symptoms, amenorrhea and bronchial asthma. Additionally, the aerial parts of this plant are frequently used in decoctions, syrups and tinctures or, alternatively, are included in pharmaceutical formulations for the treatment of cardiovascular disorders [2,5]. The plant is also used in homeopathic pharmacy for cardiac complaints, flatulence, and hyperthyroidism. Reported bioactivities of $L$. cardiaca include sedative, hypotensive and cardiotonic [2, 6-10] whereas the unique indications considered by the Committee on Herbal Medicinal Products are those of nervous tension and nervous heart complaints as palpitations [11].

Previous studies focusing the $L$. cardiaca plant have reported a large variety of compounds namely sterols, terpenes, monoterpenes, labdane diterpenes, labdanetype diterpenes, triterpenoids, pyrrholidine alkaloids, iridoides, tannins, saponins, carotenoids, polyphenolcarboxilic acids, monosaccharides, polysaccharides, caffeic acid derivatives, phenylethanoid glycosides and flavonoids [1, 2, 6-9, 12-14]. Regarding the biological properties of $L$. cardiaca, it has been shown that its alkaloid leonurine has cardioprotective and antioxidant effects. This can partly explain the in vivo anti-apoptotic activity after chronic myocardial ischemia mediated by activating the PI3K/Akt signaling pathway [15]. The same compound can exert neuroprotective activity against ischemia/reperfusion-induced mitochondrial dysfunctions in cortex [16]. Still, to the author's knowledge, the association of $L$. cardiaca phenolics to its health benefits has not been described yet.

\subsubsection{Materials and Methods}

\section{Chemicals}

Gallic acid, BHA (butylated hydroxyanisole) and DPPH ${ }^{\bullet}$ radical (2,2-diphenyl-2picrylhydrazyl) were obtained from Sigma Chemical Co (St Louis, MO, USA). Folin- 
Ciocalteu reagent and solvents were purchased from Panreac (Barcelona) and LabScan (Lisbon, Portugal). The phenolic standard compounds rosmarinic acid, rutin and verbascoside were obtained from Extrasynthese (Genay Cedex, France).

\section{Plant material and extraction of phenolic compounds}

Aerial parts (leaves, stems and flowers) of $L$. cardiaca were purchased from ERVITAL (Castro de Aire, Portugal). The procedures of phenolic extraction and purification, were similar to those described in Experimental part of the section 3.3.

\section{Determination of total phenolic compounds}

The total phenolic content of $L$. cardiaca purified ethanolic extract (PEELc) was determined according to the adapted Folin-Ciocalteu colorimetric method [17], as previous described in the Experimental part of the section 3.2.

\section{Chromatographic conditions}

This was carried out by the combination of HPLC-DAD and ESI-MS ${ }^{n}$ data following the general procedure described in the Experimental part of the section 3.3. [18]. The HPLC analysis was performed on a Knauer Smartline separation module in an endcapped Nucleosil C18 (Macherey-Nagel) column of $250 \mathrm{~mm} \times 4 \mathrm{~mm}$ id, $5 \mu \mathrm{m}$ bead diameter, that was maintained at $30^{\circ} \mathrm{C}$. The UV-Vis spectra were recorded between 220 and $500 \mathrm{~nm}$ (PDA Varian Prostar detector) and the chromatographic profiles were recorded at $340 \mathrm{~nm}$. Gradient elution was carried out with a mixture of $0.1 \%(\mathrm{v} / \mathrm{v})$ of formic acid in water (solvent $A$ ) and acetonitrile (solvent $B$ ), which were degassed and filtrated before use. The solvent gradient consisted in a series of linear gradients, starting from 10 to $20 \%$ of solvent B over 6 min, from 20 to $25 \%$ of solvent B over 12 min, from 25 to $34 \%$ over $30 \mathrm{~min}$, increasing to $100 \%$ at $37 \mathrm{~min}$ and maintaining for 3 $\mathrm{min}$, followed by the return to the initial conditions at $40 \mathrm{~min}$.

\section{Identification and quantification of phenolic compounds}

The identification of the phenolic constituents of the PEELC was carried out by the combination of HPLC-DAD and ESI-MS data, following the general procedure previously described in Experimental part of the section 3.1. As phenolic compounds determined in PEELC comprised mainly phenylethanoid glycosides and flavonols, their 
quantification were performed at $340 \mathrm{~nm}$ [19] by peak integration using the external standard method. For that, fraction 1 (caffeic acid glucoside) was quantified using rosmarinic acid as standard, fractions 2, 5 and 6 (quercetin-3-O-sophoroside, rutin and quercetin-3-O-glucoside, respectively) were quantified using rutin as a reference compound while verbascoside was used to quantified fractions $3,4,7$ and 8 (lavandulifolioside, verbascoside, leucoseptoside $A$ and leonoside $B$, respectively). Five-points calibration curves were used for each standard $(n=15)$. In more detail, for rosmarinic acid, the tested range was 0.015 to $0.173 \mathrm{mg} / \mathrm{mL}$ and the achieved equation was $y=1 E+07 x-65683$, with $R^{2}$ value of 0.9974 . The quantification limit (LQ) and detection limit (LD) of this compound were 0.031 and $0.010 \mathrm{mg} / \mathrm{mL}$, respectively. For rutin, the tested range was 0.013 to $0.2 \mathrm{mg} / \mathrm{mL}$, the equation was $\mathrm{y}=5 \mathrm{E}+06 \mathrm{x}-3044,1$, with $R^{2}$ value of 0.9981 and $L Q$ and $L D$ were 0.014 and $0.043 \mathrm{mg} / \mathrm{mL}$, respectively. The calibration curve of the phenolic standard verbascoside $\left(R^{2}=0.9985\right)$ was performed the range of 0.044 to $0.7 \mathrm{mg} / \mathrm{mL}$ with the equation $\mathrm{Y}=2 \mathrm{E}+06 \mathrm{x}+5996.8$ and LQ and LD values of 0.097 and $0.032 \mathrm{mg} / \mathrm{mL}$, respectively.

\section{Antioxidant capacity determination}

The potential antioxidant capacity of the PEELc was determined trough the DPPH radical scavenging and reducing power assays. The scavenging capacity was carried out by $\mathrm{DPPH}^{\bullet}$ radical test following the Kirby \& Schmidt procedure [20] with adaptations. Based on graphic values of $\%$ of $\mathrm{DPPH}^{\bullet}$ inhibition vs extract concentration, the $\mathrm{EC}_{50}$ (concentration of the extract able to inhibit the $50 \%$ of the $\mathrm{DPPH}^{\bullet}$ radical) of each extract was estimated. Ascorbic acid was used as a positive control.

The ability of PEELC in reducing iron (III) was assessed by the adaptation of the method described by Barros et al. [21], performed in a 48-well plate using an ELX800 Microplate Reader (BioTek Instruments, Inc., Winooski, VT, USA). The mean absorbance values were plotted against concentration, a linear regression analysis was carried out, and the $\mathrm{EC}_{50}$ value, corresponding to the extract concentration providing 0.5 of absorbance, was determined. BHA was used as positive control.

\section{Statistical analysis}

All the results were obtained from at least 3 independent experiments performed in duplicate. Data were expressed as mean $\pm S D$ 


\subsubsection{Results and Discussion}

\section{Phenolic constituents in PEELc}

The total phenolic constituents in PEELc accounted for $174.7 \pm 6.9 \mathrm{mg} \mathrm{GAE} / \mathrm{g}$ of extract (or $5.2 \mathrm{GAE} \mathrm{mg} / \mathrm{g}$ of dry plant), as estimated by the Folin Ciocalteu method. Naturally, this value is higher than those reported for polar extracts of the same specie $[1,2,7]$, since a purification step was introduced in the present work.

It is important to highlight that despite the several studies previously focusing $L$. cardiaca polar extracts [5, 9, 22-24], an accurate estimative of their phenolic content has not been reported before. This point is now herein addressed, as HPLC-DAD and ESI-MS combined analysis allowed identifying the majority of individual phenolics in the PEELc and hence, the use of more appropriate reference compounds for quantification.

Accordingly, phenolic compounds in PEELc accounted for $15 \mathrm{mg} / \mathrm{g}$ of dry plant $(500.4 \pm 49.1 \mathrm{mg} / \mathrm{g}$ of extract, Table 1$)$. Phenylethanoid glycosides were the most prevalent PEELc phenolics (Fig. 1, Table 1), with lavandulifolioside and verbascoside representing $50 \%$ and $27 \%$ of its total quantified phenolic compounds, respectively.

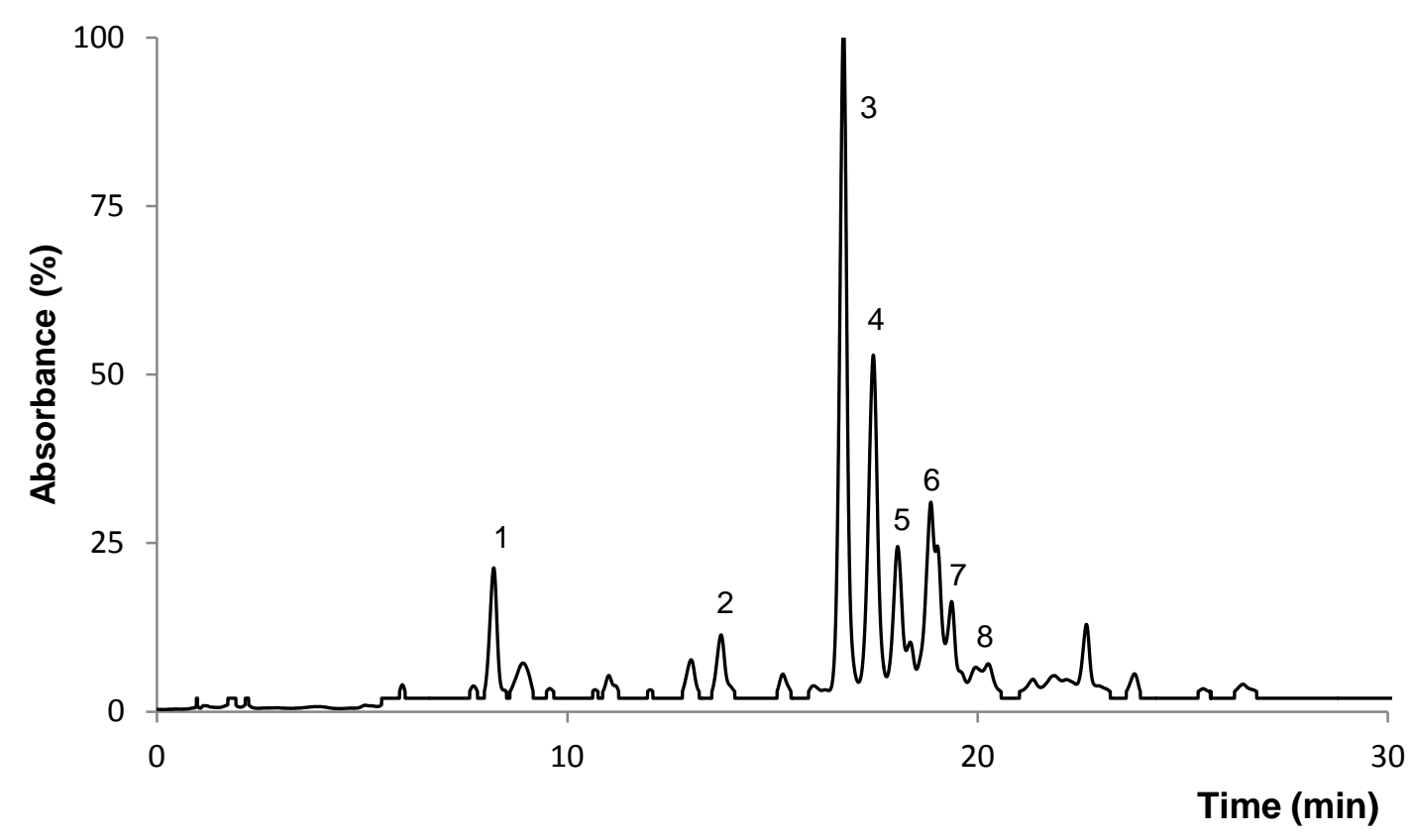

Figure 1 - Chromatographic profile at $340 \mathrm{~nm}$ of $L$. cardiaca purified ethanolic extract. 
Table 1 - Identification and quantification of the phenolic compounds in HPLC eluting peaks of L. cardiaca purified ethanolic extract through HPLC-DAD and ESI-MS combined analysis.

Phenylethanoid glycosides

\begin{tabular}{|c|c|c|c|c|c|c|c|c|c|c|c|c|c|}
\hline \multirow[t]{2}{*}{ Compound } & \multirow[t]{2}{*}{$\begin{array}{l}\mathrm{mg} / \mathrm{g} \text { of } \\
\text { extract }^{\mathrm{a}}\end{array}$} & \multirow[t]{2}{*}{$\mathbf{R T}$} & \multirow[t]{2}{*}{ UV (nm) } & \multirow[t]{2}{*}{$\begin{array}{c}{[\mathrm{M}-\mathrm{H}]^{-}} \\
(\mathrm{m} / \mathrm{z})(\%)\end{array}$} & \multicolumn{3}{|c|}{$\begin{array}{c}-\mathrm{MS}^{2}[\mathrm{M}-\mathrm{H}]^{-}(\mathrm{m} / \mathrm{z}) \\
(\%)\end{array}$} & \multicolumn{3}{|c|}{$\begin{array}{c}-\mathrm{MS}^{3}[\mathrm{M}-\mathrm{H}]^{-}(\mathrm{m} / \mathrm{z}) \\
(\%)\end{array}$} & \multicolumn{3}{|c|}{$\begin{array}{c}-\mathrm{MS}^{4}[\mathrm{M}-\mathrm{H}]^{-}(\mathrm{m} / \mathrm{z}) \\
(\%)\end{array}$} \\
\hline & & & & & -132 & -162 & -294 & -132 & -146 & Other ions & -146 & -180 & Other ions \\
\hline Lavandulifolioside & $\begin{array}{c}253.6 \pm 35.8 \\
(50.7 \%)\end{array}$ & 16.7 & 290,329 & 755 & $623(15)$ & $593(100)$ & $461(7)$ & $461(100)$ & & $315(2)$ & $\begin{array}{l}315 \\
(100)\end{array}$ & & $\begin{array}{l}297(10), \\
161(10), 135(40)\end{array}$ \\
\hline Verbascoside & $\begin{array}{c}137.4 \pm 19.9 \\
(27.4 \%)\end{array}$ & 17.5 & 290,329 & 623 & & 461 & & & $\begin{array}{l}315 \\
(100)\end{array}$ & $\begin{array}{l}297(15), \\
161(10), 143(3), \\
135(40)\end{array}$ & & $135(100)$ & $179(1), 143(2)$ \\
\hline & & & & & -132 & -176 & -146 & -132 & -146 & Other ions & -146 & & Other ions \\
\hline Leucoseptoside A & $\begin{array}{c}31.5 \pm 4.6 \\
(6.3 \%)\end{array}$ & 19.4 & ND & 637 & & $461(100)$ & $491(5)$ & & $\begin{array}{l}315 \\
(100)\end{array}$ & $135(12)$ & & & \\
\hline Leonoside B & $\begin{array}{c}25.1 \pm 4.7 \\
(5.0 \%)\end{array}$ & 20.0 & ND & 783 & $651(45)$ & $607(100)$ & & $475(100)$ & $461(5)$ & $329(2)$ & $\begin{array}{l}329 \\
(100)\end{array}$ & & $311(25), 161(20)$ \\
\hline
\end{tabular}

\section{Caffeic acid derivatives}

\begin{tabular}{|c|c|c|c|c|c|c|c|c|c|}
\hline Compound & $\begin{array}{l}\mathrm{mg} / \mathrm{g} \text { of } \\
\text { extract }^{\mathrm{a}}\end{array}$ & RT & UV (nm) & $\begin{array}{c}{[\mathrm{M}-\mathrm{H}]^{-}} \\
(\mathrm{m} / \mathrm{z})(\%)\end{array}$ & & $\begin{array}{c}-\mathrm{MS}^{2}[\mathrm{M}-\mathrm{H}]^{-}(\mathrm{m} / \mathrm{z}) \\
(\%)\end{array}$ & $\begin{array}{c}-\mathrm{MS}^{3}[\mathrm{M}-\mathrm{H}]^{-}(m / z) \\
(\%)\end{array}$ & $\begin{array}{c}-\mathrm{MS}^{4}[\mathrm{M}-\mathrm{H}]^{-}(\mathrm{m} / \mathrm{z}) \\
(\%)\end{array}$ & $\begin{array}{c}-\mathrm{MS}^{5}[\mathrm{M}-\mathrm{H}]^{-}(\mathrm{m} / \mathrm{z}) \\
(\%)\end{array}$ \\
\hline & & & & & -162 & Other ions & & & \\
\hline Caffeic acid glucoside & $\begin{array}{l}3.7 \pm 0.8 \\
(0.7 \%)\end{array}$ & 8.2 & 290,329 & $341(80)$ & $179(100)$ & $\begin{array}{l}\text { 281(10), 251(10), } \\
203(10), 161(20), \\
135(3)\end{array}$ & 135 & & \\
\hline Caffeic acid derivative & ND & 18.9 & ND & $507(15)$ & $345(5)$ & $\begin{array}{l}463(100), 323(90), \\
281(17), 251(5), 221(5), \\
179(22), 161(45)\end{array}$ & 323 & $\begin{array}{l}263(30), 221(65), 203(15), \\
179(25), 161(20), 135(10)\end{array}$ & $\mathrm{MS}^{5}[179]: 135$ \\
\hline
\end{tabular}




\section{2 | RESULTS AND DISCUSSION}

Flavonoids (quercetin and luteolin derivatives)

\begin{tabular}{|c|c|c|c|c|c|c|c|c|c|c|c|}
\hline \multirow[t]{2}{*}{ Compound } & \multirow[t]{2}{*}{$\begin{array}{l}\mathrm{mg} / \mathrm{g} \text { of } \\
\text { extract }^{\mathrm{a}}\end{array}$} & \multirow[t]{2}{*}{$\mathbf{R T}$} & \multirow[t]{2}{*}{$\mathrm{UV}(\mathrm{nm})$} & \multirow[t]{2}{*}{$\begin{array}{c}{[\mathrm{M}-\mathrm{H}]^{-}} \\
(\mathrm{m} / \mathrm{z})(\%)\end{array}$} & \multicolumn{5}{|c|}{$\begin{array}{c}-\mathrm{MS}^{2}[\mathrm{M}-\mathrm{H}]^{-}(\mathrm{m} / \mathrm{z}) \\
(\%)\end{array}$} & \multicolumn{2}{|c|}{$\begin{array}{c}-M^{3}[M-H]^{-}(m / z) \\
(\%)\end{array}$} \\
\hline & & & & & -162 & -180 & -324 & -308 & Other ions & -308 & Other ions \\
\hline Rutin-O-glucoside & ND & 8.2 & ND & $771(45)$ & $609(100)$ & $591(4)$ & & & 753(35), 301(1) & $301(100)$ & 447(2), 591(3), 343(5), \\
\hline Quercetin-3-O-sophoroside & $\begin{array}{l}5.7 \pm 1.1 \\
(1.1 \%)\end{array}$ & 13.7 & ND & 625 & $463(25)$ & $445(65)$ & $301(100)$ & & $\begin{array}{l}505(25), 300(95), \\
271(15), 255(7), \\
229(2), 179(3)\end{array}$ & & $\begin{array}{l}273(10), 179(100) \\
151(60)\end{array}$ \\
\hline Rutin & $\begin{array}{c}15.8 \pm 2.1 \\
(3.2 \%)\end{array}$ & 18.0 & $\begin{array}{c}256, \\
267,355\end{array}$ & 609 & & & & $301(100)$ & $343(10), 300(25)$ & & $\begin{array}{l}283(3), 273(15), \\
257(15), 193(5), \\
179(100), 151(60), \\
107(2)\end{array}$ \\
\hline Quercetin-3-O-glucoside & $\begin{array}{c}24.9 \pm 3.8 \\
(5.0 \%)\end{array}$ & 18.9 & $\begin{array}{c}256, \\
267,357\end{array}$ & $463(100)$ & $301(100)$ & & & & $343(3), 300(25)$ & & $\begin{array}{l}283(5), 273(17), \\
257(15), 229(4), 193 \\
(7), 179(100), 151 \\
(65), 121(2), 107(4)\end{array}$ \\
\hline Luteolin-7-O-rutinoside & ND & 18.9 & ND & $593(10)$ & & & & $285(100)$ & $461(15), 327(20)$ & & $\begin{array}{l}267(45), 257(100), \\
241(35), 229(40), \\
213(20), 199(10), \\
197(15), 151(8)\end{array}$ \\
\hline
\end{tabular}

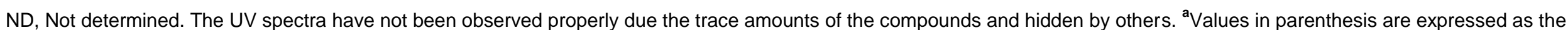
percentage of total quantified phenolic compound. 
Note that despite not quantified, these two compounds have been previously detected in L. cardiaca extracts [22, 23]. The present study allowed, however, the identification and quantification of leucoseptoside $A$ and leonoside $B$ for the first time in L. cardiaca (6.3 and $5.0 \%$ of extract of PEELc quantified phenolics, respectively). Since the ESIMS fragmentation pattern of these two compounds is scarcely described in literature, their structure was alternatively inferred on the basis of $\mathrm{MS}^{\mathrm{n}}$ data interpretation (Table 1). In fact, the MS/MS spectrum of the molecular ion at $m / z 637$ (fraction 7) showed that the product ion at $\mathrm{m} / \mathrm{z} 315$, which corresponds to a (3,4 dihydroxyphenyl)glucopyranosyl moiety, was formed upon the loss of $176 \mathrm{Da}$ (ion at $\mathrm{m} / \mathrm{z} 461$ ) and 146 Da (ion at $m / z 315$ ), e.g., the release of a feruloyl and a rhamnopyranosyl unit, respectively. Instead, the molecular ion of the major phenolic in fraction 8 (MW 784 $\mathrm{Da})$, herein assigned to leonoside B or B-(3-hydroxy, 4-methoxyphenyl)-ethyl-O- $\alpha$-Larabinopyranosyl-(1 $\rightarrow 2$ )- $\alpha$-L-rhamnopyranosyl-(1 $\rightarrow 3)-4-O-f e r u l o y l-\beta-D-$

glucopyranoside, mainly fragmented by the loss of $176 \mathrm{Da}$ (ion at $\mathrm{m} / z 607$ ) and $132 \mathrm{Da}$ (ion at $m / z 651$ ), due to the higher lability of feruloy and arabinopyranosyl linkages of this molecule, respectively. Moreover, the $\mathrm{MS}^{4}$ spectrum of the latter ion showed the loss of a rhamnopyranosyl unit, leading to the formation of the ion $[\mathrm{M}-\mathrm{H}-146]^{-}$at $\mathrm{m} / \mathrm{z}$ 329 , which correspond to the (3-hydroxy,4-methoxyphenyl)-glucopyranosyl fragment.

Besides phenylethanoid glycosides, the PEELC also contained flavonoid compounds $(10 \%)$ and caffeic acid derivatives, the latter only representing vestigial amounts of its total phenolics (Table 1). With the exception of luteolin-7-O-rutinoside (MW $594 \mathrm{Da}$ ), detected as a trace compound in fraction 6, all the remaining PEELc flavonoids were quercetin glycosidic derivatives. These enclosed isoquercitrin (quercetin-3-Oglucoside), rutin (quercetin-3-O-rutinoside), a rutin-O-glucoside (MW $772 \mathrm{Da}$ ) [25] and quercetin-3-O-sophoroside (MW $626 \mathrm{Da}$ ) [26, 27] which were detected in fractions 6,5 , 1 and 2 , respectively. From these four derivatives, only isoquercitrin and rutin were previous described to occur in $L$. cardiaca species [5, 8, 9, 23, 24]. The two caffeic acid derivatives herein detected in PEELc extract have also never been cited in the Leonurus genus, and hence, regardless their minor abundance in the extract, it is worth to highlight their presence in L. cardiaca. Indeed, to the author's knowledge, the only reported caffeic acid derivative in Leonurus plants up to present is the chlorogenic acid, which has been described as a phenolic constituent of $L$. japonicas and $L$. cardiaca species [23]. 


\section{Antioxidant properties of PEELc in chemical models}

The antioxidant activity of PEELc was estimated by means of $\mathrm{DPPH}^{\bullet}$ radical scavenging and reducing power test. The $\mathrm{EC}_{50}$ values determined in the two assays were $18.3 \pm 1.5$ and $94.7 \pm 7.0 \mu \mathrm{g} / \mathrm{mL}$, respectively. The results indicated that PEELc had 7 and 3-fold less antioxidant capacity comparing to the positive standards ascorbic acid and $\mathrm{BHA}$, respectively. Literature data focusing in $L$. cardiaca reported $\mathrm{EC}_{50}$ values for $\mathrm{DPPH}^{\bullet}$ scavenging assay between 27.3 to $144 \mu \mathrm{g} / \mathrm{mL}$ and of $20 \mu \mathrm{g} / \mathrm{mL}$ for the reducing power $[1,2,7]$.

Overall, the gathered data suggest that PEELc has considerable antioxidant properties. Further biological effects of PEELc will be described in section 3.8.

\subsubsection{References}

[1] Jafari, S.; Moradi, A.; Salaritabar, A.; Hadjiakhoondi, A.; Khanavi, M., Determination of total phenolic and flavonoid contents of Leonurus cardiaca L. in compare with antioxidant activity. Research Journal of Biological Sciences, 2010, 5(7), 484-487.

[2] Matkowski, A.; Tasarz, P.; Szypula, E., Antioxidant activity of herb extracts from five medicinal plants from Lamiaceae, subfamily Lamioideae. Journal of Medicinal Plants Research, 2008, 2(11), 321-330.

[3] Xin-Hua, L.; Hong, X.; Yi-Zhun, Z., More than a "mother-benefiting" herb: cardioprotective effect of Herba leonuri. Shengli Xuebao, 2007, 59(5), 578-584.

[4] Yang, Z. Y.; Pan, S. L.; Huo, K. K.; Wu, B. Y.; Chao, Z., Molecular Analysis of Leonurus Species in China Based on ITS and matK Sequences. American Journal of Chinese Medicine, 2011, 39(2), 411-422.

[5] Zhang, H.; Liu, X. Composition useful e.g. for treating dysmenorrhea, tonifying liver and kidney, and relieving pain, comprises e.g. Chinese Angelica, radix Paeonia alba, rhizoma Sparganium, Curcuma zedoaria, peach kernel, Leonurus and liquorice. CN102240383-A, CN102240383-A 16 Nov 2011 A61K-036/9066 201221, 2011.

[6] Matkowski, A.; Piotrowska, M., Antioxidant and free radical scavenging activities of some medicinal plants from the Lamiaceae. Fitoterapia, 2006, 77(5), 346-353.

[7] Ebrahimzadeh, M. A.; Nabavi, S. F.; Nabavi, S. M.; Eslami, B.; Asgarirad, H., In vitro antioxidant and free radical scavenging activity of Leonurus cardiaca subsp Persicus, Grammosciadium platycarpum and Onosma demawendicum. African Journal of Biotechnology, 2010, 9(51), 8865-8871.

[8] Kartnig, T.; Gruber, A.; Menzinger, S., Flavonoid-O-glycosides from the herbs of Leonurus-cardiaca. Journal of Natural Products, 1985, 48(3), 494-494. 
[9] Popescu, M. L.; Dinu, M.; Toth, O., Contributions to the pharmacognostical and phytobiological study on Leonurus cardiaca L. (Lamiaceae). Farmacia, 2009, 57(4), 424-431.

[10] Ebrahimzadeh, M. A.; Nabavi, S. M.; Nabavi, S. F., Correlation between the in vitro iron chelating activity and poly phenol and flavonoid contents of some medicinal plants. Pakistan journal of biological sciences: PJBS, 2009, 12(12), 934-8.

[11] EMEA, Assessment report on Leonurus cardiaca L., herba 2010.

[12] Masteikova, R.; Muselik, J.; Bernatoniene, J.; Majiene, D.; Savickas, A.; Malinauskas, F.; Bernatoniene, R.; Peciura, R.; Chalupova, Z.; Dvorackova, K., [Antioxidant activity of tinctures prepared from hawthorn fruits and motherwort herb]. Ceska a Slovenska farmacie : casopis Ceske farmaceuticke spolecnosti a Slovenske farmaceuticke spolecnosti, 2008, 57(1), 35-8.

[13] Ulubelen, A.; Topcu, G.; Kolak, U.; Atta ur, R., Labiatae flavonoids and their bioactivity. In Studies in Natural Products Chemistry, Elsevier: 2005; Vol. Volume 30, pp 233-302.

[14] Zhang, X.; Zhang, Z., Heart-cut two-dimensional separation method via hyphenation of micellar electrokinetic capillary chromatography and capillary zone electrophoresis using analyte focusing by micelle collapse. Journal of Chromatography $B$, 2011, 879(19), 1641-1646.

[15] Liu, X. H.; Pan, L. L.; Gong, Q. H.; Zhu, Y. Z., Leonurine (SCM-198) improves cardiac recovery in rat during chronic infarction. European Journal Pharmacology, 2010, 649(1-3), 236-241.

[16] Qi, J.; Hong, Z. Y.; Xin, H.; Zhu, Y. Z., Neuroprotective Effects of Leonurine on Ischemia/Reperfusion-Induced Mitochondrial Dysfunctions in Rat Cerebral Cortex. Biol. Pharmaceutical Bulletin, 2010, 33(12), 1958-1964.

[17] Singleton, V. L.; Rossi, J. A., Colorimetry of total phenolics with phosphomolybdicphosphotungstic acid reagents. American Journal of Enology and Viticulture, 1965, 16, 144-158.

[18] Pereira, O. R.; Domingues, M. R. M.; Silva, A. M. S.; Cardoso, S. M., Phenolic constituents of Lamium album: Focus on isoscutellarein derivatives. Food Research International, 2012, 48(1), 330-335.

[19] Galvez, M.; Martin-Cordero, C.; Houghton, P. J.; Ayuso, M. J., Antioxidant activity of Plantago bellardii All. Phytotherapy Research, 2005, 19(12), 1074-1076.

[20] Kirby, A. J.; Schmidt, R. J., The antioxidant activity of Chinese herbs for eczema and of placebo herbs - I. J. Ethnopharmacol., 1997, 56(2), 103-108.

[21] Barros, L.; Cabrita, L.; Boas, M. V.; Carvalho, A. M.; Ferreira, I. C. F. R., Chemical, biochemical and electrochemical assays to evaluate phytochemicals and antioxidant activity of wild plants. Food Chemistry, 2011, 127(4), 1600-1608.

[22] Milkowska-Leyck, K.; Filipek, B.; Strzelecka, H., Pharmacological effects of lavandulifolioside from Leonurus cardiaca. Journal of Ethnopharmacology, 2002, 80(1), 85-90. 
[23] Kuchta, K.; Ortwein, J.; Savtschenko, A.; Briel, D.; Volk, R. B.; Rauwald, H. W., Leonurus cardiaca, L. Japonicus, Leonotis leonurus: Quantitative HPLC and instrumental HPTLC determination of fourteen phenolics. Planta Medica, 2012, 78(11), PJ50.

[24] Masteikova, R.; Muselik, J.; Bernatoniene, J.; Majiene, D.; Savickas, A.; Malinauskas, F.; Bernatoniene, R.; Peciura, R.; Chalupova, Z.; Dvorackova, K., Antioxidant activity of tinctures prepared from hawthorn fruits and motherwort herb. Ceska a Slovenska farmacie : casopis Ceske farmaceuticke spolecnosti a Slovenske farmaceuticke spolecnosti, 2008, 57(1), 35-8.

[25] Vallverdu-Queralt, A.; Jauregui, O.; Medina-Remon, A.; Andres-Lacueva, C.; Lamuela-Raventos, R. M., Improved characterization of tomato polyphenols using liquid chromatography/electrospray ionization linear ion trap quadrupole Orbitrap mass spectrometry and liquid chromatography/electrospray ionization tandem mass spectrometry. Rapid Communications in Mass Spectrometry, 2010, 24(20), 2986-2992.

[26] Ferreres, F.; Taveira, M.; Pereira, D. M.; Valentao, P.; Andrade, P. B., Tomato (Lycopersicon esculentum) Seeds: New Flavonols and Cytotoxic Effect. Journal of Agricultural and Food Chemistry, 2010, 58(5), 2854-2861.

[27] Ferreres, F.; Sousa, C.; Valentao, P. c.; Pereira, J. A.; Seabra, R. M.; Andrade, P. B., Tronchuda cabbage flavonoids uptake by Pieris brassicae. Phytochemistry, 2007, 68(3), 361-367. 


\subsection{ROS SCAVENGING AND hePATOPROTECTIVE ACTIVITIES OF MENTHA AQUATICA L. AND LAVANDULA DENTATA L.}

Mentha aquatica L. and Lavandula dentata L. are two Lamiaceae species that are consumed as spices or in food and beverages manufacturing. This mint species has been used in traditional medicine for the treatment of external inflammation, as mouthwash and to gargle for treating sore throats, while the lavender species is mainly used in the form of tea to treat diabetes, colds and renal colics problems. The present study aimed to determine the exact phenolic composition of the two extracts and also evaluate their antioxidant and cytoprotective effects.

The ethanolic extracts of the two plants are rich in rosmarinic acid $(64 \pm 2$ and $68 \pm 3$ $\mathrm{mg} / \mathrm{g}$ of the purified ethanolic extract of $M$. aquatica and $L$. dentata, respectively). The $M$. aquatica extract also contained large amounts of other polyphenols, being the most abundant the eriodictyol-7-O-rutinoside $(145 \pm 6 \mathrm{mg} / \mathrm{g})$. Albeit with some differences, both purified ethanolic extracts exhibited significant antioxidant abilities, as established by $\mathrm{DPPH}^{\bullet}$ and reducing power assays, as well as in a model of oxidative stress induced by potassium dichromate in HepG2 cells. Concretely, lower $\mathrm{EC}_{50}$ values were found for $M$. aquatica purified ethanolic extract in chemical assays, while L. dentata purified ethanolic extract had higher capacity in counteracting the ROS formation induced by potassium dichromate in human hepatoblastoma HepG2 cells. The protection corresponded to $30 \%$ for $25 \mu \mathrm{M}$ DK-induced toxicity and treatment with $L$. dentata extract at $50 \mu \mathrm{g} / \mathrm{mL}$. In turn, $M$. aquatica purified ethanolic extract induced an effective cytoprotective effect (13\%), as measured by the MTT test. Overall, these results provide new important information for the chemical and pharmacological characterization of $M$. aquatica and $L$. dentata purified ethanolic extracts, in view to add value to the two plants species.

The results obtained in this section of the Doctoral Thesis have been used to write the manuscript entitled "ROS scavenging and hepatoprotective activities of Mentha aquatica L. and Lavandula dentata L." which is under preparation. 


\section{ROS scavenging and hepatoprotective activities of Mentha aquatica $\mathrm{L}$. and}

\section{Lavandula dentata $\mathrm{L}$.}

Olívia R. Pereira ${ }^{1,2}$, María J. Perez ${ }^{3,4}$, Rocio I. R. Macias ${ }^{4}$, Maria R. M. Domingues ${ }^{5}$, Jose J. G. Marin ${ }^{4}$, Susana M. Cardoso ${ }^{1,6, *}$

${ }^{1}$ CERNAS, Escola Superior Agrária, Instituto Politécnico de Coimbra, Bencanta, 3040316 Coimbra, Portugal;

${ }^{2}$ Departamento de Tecnologias de Diagnóstico e Terapêutica, Escola Superior de Saúde, Instituto Politécnico de Bragança, Av. D. Afonso V, 5300-121 Bragança, Portugal;

${ }^{3}$ Hospital Universitario de Salamanca, Instituto de Estudios de Ciencias de la Salud de Castilla y León, Salamanca, CIBERehd, Spain;

${ }^{4}$ Laboratory of Experimental Hepatology and Drug Targeting (HEVEFARM), Biomedical Research Institute of Salamanca (IBSAL), CIBERehd, University of Salamanca, Spain;

${ }^{5}$ Departamento de Química \& QOPNA, Universidade de Aveiro, 3810-193 Aveiro, Portugal;

${ }^{6}$ CIMO, Escola Superior Agrária, Instituto Politécnico de Bragança, Campus de Santa Apolónia - Apartado 1038 - 5301-854, Bragança, Portugal.

\section{*Corresponding author:}

Susana M. Cardoso, CERNAS - Escola Superior Agrária, Instituto Politécnico de Coimbra, Bencanta, 3040-316 Coimbra, Portugal. Telephone: +351 239 802940; Fax: +351273239 802979. E-mail: $\underline{\text { scardoso@esac.pt }}$ 


\begin{abstract}
Mentha aquatica L. and Lavandula dentata L. are two edible Lamiaceae species enriched in rosmarinic acid. In this study, this phenolic compound accounted for $64 \pm 2$ and $68 \pm 3 \mathrm{mg} / \mathrm{g}$ of the purified ethanolic extract of M. aquatica and L. dentata, respectively, however, these amounts represented distinct phenolic extract percentages ( $21 \%$ and $72 \%$ of total phenolics, respectively). Moreover, the $M$. aquatica extract contained high amounts of eriodictyol-7- $O$-rutinoside $(145 \pm 6 \mathrm{mg} / \mathrm{g})$. The two extracts exhibited distinct antioxidant and hepatoprotective abilities. Lower $\mathrm{EC}_{50}$ values were found for $M$. aquatica purified ethanolic extract in the 2,2-diphenyl-2-picrylhydrazyl and reducing power assays, but $L$. dentata purified ethanolic extract had higher capacity in counteracting the reactive oxygen species formation induced by potassium dichromate in human hepatoblastoma HepG2 cells. In turn, $M$. aquatica purified ethanolic extract $(50 \mu \mathrm{g} / \mathrm{mL})$ induced an effective cytoprotective effect, as measured by the MTT test. Overall, the biological properties herein described can contribute to add value to the two plants.
\end{abstract}

Keywords: Mentha aquatica L.; Lavandula dentata L.; phenolic compounds; HPLCDAD; ESI-MS; human hepatoblastoma HepG2 cells 


\section{Introduction}

Lamiaceae family encloses many plant species with applications on food, pharmaceutical and cosmetics industries [1-3]. Several studies have recently described that a large proportion of them possess different beneficial health properties, including antiproliferative, anti-tumoral, anti-inflammatory, antimicrobial, analgesic and neuroprotective properties $[4,5]$, which have been related to the antioxidant ability of their polyphenols [6-8].

Mentha aquatica L. has been consumed as tea or as a food component of beverages, salads or cooked foods, and has been used in traditional medicine for the treatment of external inflammation, as mouth-wash and to gargle for treating sore throats $[9,10]$. To our knowledge, the antioxidant ability of $M$. aquatica has been demonstrated in vitro by assessing the free radical scavenging activity against DPPH, and by evaluating the lipid peroxidation protective activity [10]. According to literature data, the phenolic composition of M. aquatica includes phenolic acids (caffeic acid, its esters nepetoidin A and rosmarinic acid), the flavones luteolin and its $O$-glucoside, apigenin and apigenin-7$O$-rutinoside, the methylated flavones pebrellin, gardenin B and salvigenin and the flavanone eriocitrin [11-14].

Plants of Lavandula genus have been widely used as spices in food manufacturing of ice cream, candy, baked goods, chewing gum and beverages [15]. Moreover, the species Lavandula dentata L. is also consumed in the tea form to counteract diabetes, colds and renal colics [16]. The biological activities of $L$. dentata have been mainly exploited for essential oils fractions. To our knowledge, only one aqueous extract of this plant species has been tested for anti-tyrosinase and antioxidant activities, the latter through the DPPH assay [17]. Likewise, the phenolic composition of this plant is scarcely reported 
and comprises rosmarinic acid, luteolin, apigenin and glycosidic forms of these two flavones [18].

Despite the mentioned applications of $M$. aquatica and L. dentata, their usage is very limited compared to other medicinal plants of their respective genus. The present study intends to improve the knowledge of the phenolic composition of $M$. aquatica and $L$. dentata species, and simultaneously evaluate their potential antioxidant and cytoprotective effects with the aim of contributing to the valorization of the two plants as agents potentially useful for food and pharmaceutical industries.

\section{Material and methods}

\subsection{Materials}

Butylated hydroxyanisole (BHA) and 2,2-diphenyl-2-picrylhydrazyl (DPPH) radical were obtained from Sigma Chemical Co (St Louis, MO, USA). 3-(4,5-dimethylthiazol2-yl)-2,5-diphenyltetrazolium bromide (MTT), dichlorofluorescein-diacetate and potassium dichromate were purchased from Sigma-Aldrich (Madrid, Spain). Phenolic standards were obtained from Extrasynthese (Genay Cedex, France). Aerial parts (leaves and stems) of $M$. aquatica and flowers of $L$. dentata were purchased from ERVITAL (Castro de Aire, Portugal).

\subsection{Obtention of plant extracts}

Five g of plant material was grounded and defatted with $n$-hexane. The defatted residue was then extracted with $80 \%$ ethanolic solution (v/v), and purified using Sep-Pak C18 cartridges, as previously described [19]. The extraction and purification steps were performed three times. 


\subsection{Analysis of phenolic compounds}

Identification and quantification of phenolic compounds were carried out by the combination of HPLC-DAD and ESI-MS ${ }^{\mathrm{n}}$ data following a general procedure previously described [19]. HPLC analysis was performed on a Knauer Smartline separation module in an end-capped Nucleosil C18 (Macherey-Nagel) column of 250 $\mathrm{mm} \times 4 \mathrm{~mm}$ id, $5 \mu \mathrm{m}$ bead diameter, that was maintained at $30^{\circ} \mathrm{C}$. Gradient elution was carried out with a mixture of $0.1 \%(v / v)$ of formic acid in water (solvent A) and acetonitrile (solvent B). The solvent gradient started from 10 to $20 \%$ of solvent B over 6 min, from 20 to $25 \%$ of solvent B over $12 \mathrm{~min}$, from 25 to $34 \%$ over $30 \mathrm{~min}$, increasing to $100 \%$ at $37 \mathrm{~min}$. The flow rate used was $1 \mathrm{~mL} / \mathrm{min}$. The $\mathrm{UV}-\mathrm{V}$ is spectra were recorded between 220 and $500 \mathrm{~nm}$ (PDA Varian Prostar detector) and the chromatographic profiles were recorded at $280 \mathrm{~nm}$. Phenolic identification in each HPLC peak was achieved by comparison of retention time, UV-Vis spectra and $\mathrm{MS}^{\mathrm{n}}$ spectra data with those of available reference standards or alternatively, with those registered in literature. Note that phenolic compounds which were detected in $\mathrm{MS}^{\mathrm{n}}$ analysis as a minor $[\mathrm{M}-\mathrm{H}]-$ ion and were not simultaneously detected by UV-spectra analysis, were herein considered as trace components. Additionally, the quantification of the majority of the compounds in both plant extracts was performed at $280 \mathrm{~nm}$, by peak integration using the external standard method, with the most close reference compound available. The calibration curves of the standards used for quantification of the distinct phenolic compounds in the two purified ethanolic extracts are shown in Table 1 . The linearity of the calibration curves, the regression coefficient $\left(\mathrm{R}^{2}\right)$ and the detection and quantification limits (LOD and LOQ, respectively) are also represented. LOD and LOQ were determined as 3.3 and 10 times the value of the regression error divided by the slope, respectively [20,21]. 


\subsection{DPPH test}

The ability of each extract to scavenge DPPH free radicals was carried out following the Kirby \& Schmidt procedure [22] with adaptations (Pereira et al., 2013). Based on graphic values of percentage of DPPH inhibition $v s$ extract concentration, the $\mathrm{EC}_{50}$ (concentration of the extract able to inhibit the 50\% of the DPPH radical) of each extract was estimated. Ascorbic acid was used as a positive control.

\subsection{Reducing power test}

The ability of the purified ethanolic extracts to reduce iron (III) to iron (II) was assessed by an adaptation of the method described by Barros et al [23]. The mean absorbance values were plotted against concentration, a linear regression analysis was carried out, and the $\mathrm{EC}_{50}$ value, corresponding to the extract concentration providing 0.5 of absorbance, was determined. BHA was used as a positive control.

\subsection{Cell cultures}

HepG2 cells, from human hepatoblastoma (HB-8065, ATCC), were cultured in MEM medium supplemented with $1 \mathrm{mM}$ sodium pyruvate, $26.2 \mu \mathrm{M}$ sodium bicarbonate, $10 \%$ (v/v) fetal calf serum, penicillin $\mathrm{G}(20 \mathrm{U} / \mathrm{mL})$, streptomycin $(0.02 \mathrm{mg} / \mathrm{mL})$ and amphotericin B $(0.05 \mu \mathrm{g} / \mathrm{mL})$. The cells were maintained at $37^{\circ} \mathrm{C}$ in an atmosphere of $95 \%$ air $/ 5 \% \mathrm{CO}_{2}$ and with $90-95 \%$ humidity.

\subsection{Determination of cell viability by MTT assay}

Cell viability was estimated by the formazan formation from the tetrazolium salt (MTT) by living cells as previously described [24]. HepG2 cells were plated onto 96 wellplates $(15,000$ cells/well, $100 \mu \mathrm{L}$ medium) and exposed to $1.5 \mu \mathrm{M}$ potassium 
dichromate alone or in presence of extracts for $72 \mathrm{~h}$. This condition was selected based on preliminary experiments (Pereira et al., 2013) to determine the potassium dichromate concentration able to reduce cell viability by approximately $30 \%$. Cell viability was calculated as the percentage of living cells compared to untreated (control) cells.

\subsection{Determination of intracellular ROS production by flow cytometry}

HepG2 cells were seeded in 6 well-plates $(100,000$ cells/plate, $2 \mathrm{~mL}$ medium). The intracellular ROS formation was evaluated after the exposure of cells, for $48 \mathrm{~h}$, to $5 \mu \mathrm{M}$ or $25 \mu \mathrm{M}$ potassium dichromate alone or in presence of $M$. aquatica or L. dentata purified ethanolic extracts (final concentrations of $50 \mu \mathrm{g} / \mathrm{mL}$ ). These conditions were previously pre-established in our group to produce an effective ROS-increment in these cells (data not shown). After $48 \mathrm{~h}$, the medium was replaced by RPMI culture medium containing $5 \mu \mathrm{g} / \mathrm{mL}$ of 2,7-dichlorofluorescein diacetate and incubated for $30 \mathrm{~min}$. Cells were harvested and ROS generation was measured and analyzed in a cytometer FACSort flow cytometer (BD Biosciences, San Jose, CA, USA) using the CellQuest software (BD Biosciences). The values were normalized to the percentage of ROS formation in untreated cells.

\subsection{Statistical analysis}

Data were expressed as mean \pm SEM of the number of experiments as indicated in the figure legends. The comparison between groups was performed by one-way ANOVA, followed by Dunnett's post-hoc test.

\section{Results and discussion}




\subsection{Phenolic constituents in $M$. aquatica and $L$. dentata purified ethanolic extracts}

In the present work we used MS spectrometry, together with HPLC-DAD for qualitative (Table 2) and quantitative analysis of phenolic compounds in $M$. aquatica and $L$. dentata. Total phenols accounted for $303 \pm 8$ and $94 \pm 2 \mathrm{mg} / \mathrm{g}$ of extract in $M$. aquatica and $L$. dentata purified ethanolic extracts, respectively (Table 3). The two extracts contained similar amounts of rosmarinic acid (Fig. 1, Table 3), but this compound represented $21 \%$ and $72 \%$ of the total quantified phenols in $M$. aquatica and L. dentata purified ethanolic extracts, respectively. The amount of rosmarinic acid herein found in the $M$. aquatica purified ethanolic extract $(64.2 \pm 2.4 \mathrm{mg} / \mathrm{g}$ extract or $7.3 \pm 0.3 \mathrm{mg} / \mathrm{g}$ dry plant) is in-between the quantities previously described for the same plant $[11,25]$ and fits well on the contents found in Mentha plants $(1.1$ to $19.1 \mathrm{mg} / \mathrm{g}$ of dry plant) $[11,26]$. In turn, the amount of rosmarinic acid in the L. dentata purified ethanolic extract $(67.8 \pm$ $3.3 \mathrm{mg} / \mathrm{g}$ extract or $4.5 \pm 0.2 \mathrm{mg} / \mathrm{g}$ dry plant) was considerable higher than that reported for L. intermedia and L. angustifolia (1.2 and $1.7 \mathrm{mg} / \mathrm{g}$ of dry plant, respectively) [25, 27]. Note that, despite previously reported in L. dentata [18], the amount of rosmarinic acid in this species has not been deliverable up to this date.

The $M$. aquatica purified ethanolic extract also contained significant amounts of other phenolics (Fig. 1 and Table 3), mainly flavanones. Together, these phenolics represented approximately $65 \%$ of total phenols in the extract, which is in accordance with previous literature data reporting the prevalence of this subclass of compounds in Mentha plants [26, 28]. Eriodictyol- $O$-rutinoside (peak $1,[\mathrm{M}-\mathrm{H}]^{-}$ion at $\mathrm{m} / z$ 595, Table 2) was identified as the main flavanone in $M$. aquatica purified ethanolic extract, accounting for almost half of the total quantified phenolics $(144.6 \pm 6.2 \mathrm{mg} / \mathrm{g}$ extract or $16.4 \pm 0.7 \mathrm{mg} / \mathrm{g}$ of dry plant). Although this value is higher than that previously reported for this species $(2.1 \mathrm{mg} / \mathrm{g}$ of dry plant) [11, 26] overall, it fits on the average 
amounts found in the Mentha genus [26, 28]. Other previously described flavanones (corresponding to the ion at $m / z, 449$ ) and flavones glycosides (e.g. luteolin-7-Oglucoside and apigenin-7-O-rutinoside) were also detected in minor amounts in the $M$. aquatica extract (Table 2 and Table 3). Note still that the present study also allowed us to detect for the first time in $M$. aquatica, the flavone luteolin-7-O-rutinoside $(4.9 \pm 0.3$ $\mathrm{mg} / \mathrm{g}$ of dry plant), the flavanone naringenin-7-O-rutinoside and the bioflavonol hesperitin-7-O-rutinoside.

L. dentata purified ethanolic extract main phenolics comprised the luteolin-7-Oglucuronide (28\% of the total quantified phenolic components) and rosmarinic acid ( $72 \%$ of the total quantified phenolic components). Besides those, the only detectable phenolic compound in the L. dentata purified ethanolic extract was an apigenin derivative, which has not been previously reported in Lavandula genus. This compound was detected in low amounts in peak 6 and was tentatively assigned to apigenin-7-O(acetyl)glucoside, in accordance to its fragmentation pattern $(473 \rightarrow 413,269)$ plus its UV spectra data.

\subsection{Antioxidant properties of $M$. aquatica and $L$. dentata purified ethanolic extracts in chemical models}

Both DPPH radical scavenging and reducing power assays were performed in $M$. aquatica and L. dentata purified ethanolic extracts, as a first approach to evaluate their antioxidant abilities. $M$. aquatica and $L$. dentata purified ethanolic extracts showed DPPH $\mathrm{EC}_{50}$ values of $8.1 \pm 0.7$ and $11.6 \pm 0.6 \mu \mathrm{g} / \mathrm{mL}$, respectively, and their scavenging ability was 3 to 5 times lower than that of the ascorbic acid. Note that these $\mathrm{EC}_{50}$ values are significantly lower than those previously described for $M$. aquatica 
(27.1 and $29.0 \mu \mathrm{g} / \mathrm{mL}[10,29])$ and L. dentata extracts $(48.7 \mu \mathrm{g} / \mathrm{mL}[17])$, which can result from the herein applied purification process.

Regarding the reducing power, the obtained $\mathrm{EC}_{50}$ values were $51.9 \pm 7.2$ and $78.9 \pm 1.5$ $\mu \mathrm{g} / \mathrm{mL}$, respectively, for $M$. aquatica and L. dentata purified ethanolic extracts. These corresponded to a 2- to 3-fold less potency than that of BHA (which is a potent synthetic antioxidant) in reducing iron (III) to iron (II). Overall, both $M$. aquatica and $L$. dentata purified ethanolic extracts showed relevant antioxidant activity in the two chemical tests, although DPPH-scavenging ability and electron-donating antioxidant activity was superior in the $M$. aquatica purified ethanolic extract.

\subsection{Protective activities of $M$. aquatica and $L$. dentata purified ethanolic extracts in}

\section{HepG2 cells}

HepG2 cells are commonly used for evaluating the protective or cytotoxic effects of compounds/extracts in liver cells because they retain many of the specialized functions of healthy human hepatocytes [30]. On the other hand, potassium dichromate has been previously shown to induce toxicity in several biological models, including the human hepatoblastoma HepG2 cell line [7, 31]. It is known that this compound enters rapidly into the cells and activates intracellular reduction pathways. These events lead to a decline in membrane potential and a massive production of ROS, such as hydrogen peroxide and superoxide anion radical, resulting in oxidative damage and a cascade of cellular events, such as lipid peroxidation, DNA breakdown and induction of apoptosis through caspases activation [32].

As a first approach to this part of the work, we investigated the effect of a broad range of extract concentrations (1-200 $\mu \mathrm{g} / \mathrm{mL})$ of $M$. aquatica and L. dentata on HepG2 cell survival, after exposure for $72 \mathrm{~h}$, by the MTT method. As can be observed in Fig. 2, the 
doses of $100 \mu \mathrm{g} / \mathrm{mL}$ or higher were toxic for the cells, but from $1-50 \mu \mathrm{g} / \mathrm{mL}$ these extracts did no affect cell viability. Hence, $50 \mu \mathrm{g} / \mathrm{mL}$ was selected for further investigation of the potential ROS scavenging and cytoprotective activities of the two plant extracts.

Potassium dichromate increased intracellular levels of ROS in HepG2 cells in a concentration-dependent manner (Fig. 3A); ROS production was 1.7 -fold for $5 \mu \mathrm{M}$ and 2.4-fold for $25 \mu \mathrm{M}$ potassium dichromate. We next investigated the protective effect of M. aquatica and $L$. dentata purified ethanolic extracts in intracellular ROS production induced by potassium dichromate, and found that ROS scavenging ability was higher for $L$. dentata than for M. aquatica. In fact, L. dentata significantly reduced ROS levels in HepG2 cells under basal conditions, and in cells exposed to $5 \mu \mathrm{M}$ or $25 \mu \mathrm{M}$ potassium dichromate by about 35, 20 and 30\%, respectively. On the contrary, $M$. aquatica purified ethanolic extract only significantly decreased ROS production induced by the highest concentration of potassium dichromate (approximately 25\%). The ROS reducing ability of $M$. aquatica or $L$. dentata purified ethanolic extracts in cells has not been previous described. To our knowledge, from these two plants genus, ROS scavenging potential, in particular for superoxide anion and hydroxyl radical, has only been reported for L. stoechas in neutrophils [5].

When evaluating the protective effect of $M$. aquatica or L. dentata purified ethanolic extracts on the potassium dichromate-induced ROS increment, one should consider that rosmarinic acid and luteolin are the main components of $L$. dentata, and are present in similar amounts in the two extracts. Both phenols have been shown to exhibit high ROS scavenging activity in several cell models, including the HepG2 cells [33]. In fact, previous studies performed by Pereira et al [7] have demonstrated that rosmarinic acid and luteolin could efficiently counteract potassium dichromate-induced ROS increment 
production. Hence, it is feasible that these phenols could be associated, at least, in part, to the ROS scavenging ability observed for the M. aquatica or L. dentata purified ethanolic extracts.

Two other phenols present in M. aquatica, eriodictyol and naringenin, also possess high ROS scavenging activity in HepG2 cells (Pereira et al., 2013) (about 50\% of protection at $50 \mu \mathrm{g} / \mathrm{mL}$ ) however, the effect of this purified ethanolic extract was lower than that of L. dentata. Hence, other phenolic or non-phenolic extract components must also contribute to the final ROS reducing ability of each extract.

Interestingly, the antioxidant ability of the extracts was not directly associated to their cytoprotective capacity. As can be observed in Fig. 3B, a moderate cytoprotective effect was observed in HepG2 cells exposed to $1.5 \mu \mathrm{M}$ potassium dichromate together with $M$. aquatica purified ethanolic extract $(50 \mu \mathrm{g} / \mathrm{mL})$ but not together with $L$. dentata extract, despite the later presents higher ROS scavenging activity. These results suggest that the protective effect observed for $M$. aquatica purified ethanolic extract is mainly related to a ROS-independent scavenging mechanism. Since this extract is rich in an eriodictyol derivative, and this aglycone has been shown to improve the cells survival [7], as well to potently inhibit several apoptotic important steps, including the cleavage of procaspase- 3 or pro-caspase- 9 and the release of cytochrome $C$ [34], it is possible that the herein observed protection for the $M$. aquatica purified ethanolic is partially mediated through anti-apoptotic effects of this compounds. Future work focusing the effect of $M$. aquatica purified ethanolic extract on apoptotic-associated processes will help to further understand the observed M. aquatica hepatoprotection. 


\section{Conclusion}

This study provides new important information for the chemical and pharmacological characterization of $M$. aquatica and $L$. dentata purified ethanolic extracts, in view to add value to the two plants species. Both plant extracts are rich in rosmarinic acid and luteolin glycosides but $M$. aquatica also contained large amounts of other polyphenols, in particular eriodictyol-7- $O$-rutinoside. Besides this, both plant purified ethanolic extracts have considerable antioxidant abilities, as established by DPPH and reducing power assays, as well as in a model of chemical stress induced by potassium dichromate in HepG2 cells. Although it is likely that rosmarinic acid and luteolin are associated to their antioxidant properties, other extract constituents must also contribute to this effect. Moreover, the cytoprotective activity observed in the presence of $M$. aquatica purified ethanolic extract seems to be related to a ROS-independent scavenging mechanism, which deserves further investigation. In conclusion, the present work suggest that the studied plants might be important dietary sources of natural antioxidants and that can be consumed for prevention of dysfunctions related with oxidative stress. Further studies are now necessary to clarify the exact contribution of phenolic compounds in the described effects.

\section{Acknowledgements}

The authors acknowledge the financial support provided by the FCT to CERNAS (project PEst-OE/AGR/UI0681/2011) and of the FCT as well as FSE (III Quadro Comunitário de Apoio) to QOPNA (project PEst-C/QUI/UI0062/2011), REDE/1504/REM/2005 (that concerns the Portuguese Mass Spectrometry Network).

Olívia R. Pereira was supported by a PhD grant (SFRH/PROTEC/49600/2009). 
This study was also supported in part by the Ministerio de Ciencia e Innovación (Grant SAF2010-15517); the Instituto de Salud Carlos III, FIS (Grant PI11/0337) and the Junta de Castilla y León (Grants SA023A11-2, SA070A11-2 and BIO/03/SA23/11), Spain. 


\section{References}

[1] Theodoridis, S.; Stefanaki, A.; Tezcan, M.; Aki, C.; Kokkini, S.; Vlachonasios, K. E., DNA barcoding in native plants of the Labiatae (Lamiaceae) family from Chios Island (Greece) and the adjacent Cesme-Karaburun Peninsula (Turkey). Molecular Ecology Resources, 2012, 12(4), 620-633.

[2] Lee, J., Caffeic acid derivatives in dried Lamiaceae and Echinacea purpurea products. Journal of Functional Foods, 2010, 2(2), 158-162.

[3] Lee, J.; Scagel, C. F., Chicoric acid levels in commercial basil (Ocimum basilicum) and Echinacea purpurea products. Journal of Functional Foods, 2010, 2(1), 77-84.

[4] Tadic, V. M.; Jeremic, I.; Dobric, S.; Isakovic, A.; Markovic, I.; Trajkovic, V.; Bojovic, D.; Arsic, I., Anti-inflammatory, Gastroprotective, and Cytotoxic Effects of Sideritis scardica Extracts. Planta Med., 2012, 78(5), 415-427.

[5] Amira, S.; Dade, M.; Schinella, G.; Rios, J. L., Anti-inflammatory, anti-oxidant, and apoptotic activities of four plant species used in folk medicine in the Mediterranean basin. Pak. J. Pharm. Sci., 2012, 25(1), 65-72.

[6] Krishnaiah, D.; Sarbatly, R.; Nithyanandam, R., A review of the antioxidant potential of medicinal plant species. Food and Bioproducts Processing, 2011, 89(3), 217-233.

[7] Pereira, O. R.; Macias, R. I. R.; Perez, M. J.; Marin, J. J. G.; Cardoso, S. M., Protective effects of phenolic constituents from Cytisus multiflorus, Lamium album L. and Thymus citriodorus on liver cells. Journal of Functional Foods, 2013, in press.

[8] Deng, G. F.; Lin, X.; Xu, X. R.; Gao, L. L.; Xie, J. F.; Li, H. B., Antioxidant capacities and total phenolic contents of 56 vegetables. Journal of Functional Foods, 2013, 5(1), 260-266. 
[9] Olsen, H. T.; Stafford, G. I.; van Staden, J.; Christensen, S. B.; Jaeger, A. K., Isolation of the MAO-inhibitor naringenin from Mentha aquatica L. $J$. Ethnopharmacol., 2008, 117(3), 500-502.

[10] Conforti, F.; Sosa, S.; Marrelli, M.; Menichini, F.; Statti, G. A.; Uzunov, D.; Tubaro, A.; Menichini, F.; Della Loggia, R., In vivo anti-inflammatory and in vitro antioxidant activities of Mediterranean dietary plants. Journal of Ethnopharmacology, 2008, 116(1), 144-151.

[11] Kosar, M.; Dorman, H. J. D.; Baser, K. H. C.; Hiltunen, R., Screening of free radical scavenging compounds in water extracts of Mentha samples using a postcolumn derivatization method. Journal of Agricultural and Food Chemistry, 2004, 52(16), 5004-5010.

[12] Grayer, R. J.; Eckert, M. R.; Veitch, N. C.; Kite, G. C.; Marin, P. D.; Kokubun, T.; Simmonds, M. S. J.; Paton, A. J., The chemotaxonomic significance of two bioactive caffeic acid esters, nepetoidins $\mathrm{A}$ and $\mathrm{B}$, in the Lamiaceae. Phytochemistry, 2003, 64(2), 519-528.

[13] Zaidi, F.; Voirin, B.; Jay, M.; Viricel, M. R., Free flavonoid aglycones from leaves of Mentha pulegium and Mentha suaveolens (Labiatae). Phytochemistry, 1998, 48(6), 991-994.

[14] Voirin, B.; Bayet, C.; Faure, O.; Jullien, F., Free flavonoid aglycones as markers of parentage in Mentha aquatica, M. citrata, M. spicata and M. x piperita. Phytochemistry, 1999, 50(7), 1189-1193.

[15] Kim, N.-S.; Lee, D.-S., Comparison of different extraction methods for the analysis of fragrances from Lavandula species by gas chromatographyâ€"mass spectrometry. Journal of Chromatography A, 2002, 982(1), 31-47. 
[16] Gamez, M. J.; Zarzuelo, A.; Risco, S.; Utrilla, P.; Jimenez, J., Hypoglycemic activity in various species of the genus Lavandula. Pharmazie, 1988, 43(6), 441442.

[17] Sariri, R.; Seifzadeh, S.; Sajedi, R. H., Anti-tyrosinase and antioxidant activity of Lavandula sp. extracts. Pharmacologyonline, 2009, 2, 413-420.

[18] Upson, T. M.; J. Grayer, R. e.; Greenham, J. R.; A. Williams, C.; Al-Ghamdi, F.; Chen, F.-H., Leaf flavonoids as systematic characters in the genera Lavandula and Sabaudia. Biochemical Systematics and Ecology, 2000, 28(10), 991-1007.

[19] Pereira, O. R.; Domingues, M. R. M.; Silva, A. M. S.; Cardoso, S. M., Phenolic constituents of Lamium album: Focus on isoscutellarein derivatives. Food Research International, 2012, 48(1), 330-335.

[20] Ermer, J.; Miller, J. M., Method Validation in Pharmaceutical Analysis: A Guide to Best Practice. Weinheim: WILEY-VCH Verlag GmbH \& Co: 2005.

[21] Snyder, L.; Kirkland, J.; Dolan, J., Introduction to Modern Liquid Chromatography. John Wiley \& Sons, Inc: New Jersey, 2010.

[22] Kirby, A. J.; Schmidt, R. J., The antioxidant activity of Chinese herbs for eczema and of placebo herbs -- I. J. Ethnopharmacol., 1997, 56(2), 103-108.

[23] Barros, L.; Cabrita, L.; Boas, M. V.; Carvalho, A. M.; Ferreira, I. C. F. R., Chemical, biochemical and electrochemical assays to evaluate phytochemicals and antioxidant activity of wild plants. Food Chem., 2011, 127(4), 1600-1608.

[24] Briz, O.; Serrano, M. A.; Macias, R. I. R.; Marin, J. J. G., Overcoming cisplatin resistance in vitro by a free and liposome-encapsulated bile acid derivative: BametR2. International Journal of Cancer, 2000, 88(2), 287-292. 
[25] Shekarchi, M.; Hajimehdipoor, H.; Saeidnia, S.; Gohari, A. R.; Hamedani, M. P., Comparative study of rosmarinic acid content in some plants of Labiatae family. Pharmacogn. Mag., 2012, 8(29), 37-41.

[26] Dorman, H. J. D.; Kosar, M.; Kahlos, K.; Holm, Y.; Hiltunen, R., Antioxidant properties and composition of aqueous extracts from Mentha species, hybrids, varieties, and cultivars. Journal of Agricultural and Food Chemistry, 2003, 51(16), 4563-4569.

[27] Torras-Claveria, L.; Jauregui, O.; Bastida, J.; Codina, C.; Viladomat, F., Antioxidant activity and phenolic composition of lavandin (Lavandula x intermedia emeric ex loiseleur) waste. Journal of Agricultural and Food Chemistry, 2007, $55(21), 8436-8443$.

[28] Areias, F. M.; Valentao, P.; Andrade, P. B.; Ferreres, F.; Seabra, R. M., Phenolic fingerprint of peppermint leaves. Food Chem., 2001, 73(3), 307-311.

[29] Lopez, V.; Akerreta, S.; Casanova, E.; Garcia-Mina, J. M.; Cavero, R. Y.; Calvo, M. I., In vitro antioxidant and anti-rhizopus activities of lamiaceae herbal extracts. Plant Foods for Human Nutrition, 2007, 62(4), 151-155.

[30] Mersch-Sundermann, V.; Knasmuller, S.; Wu, X.-j.; Darroudi, F.; Kassie, F., Use of a human-derived liver cell line for the detection of cytoprotective, antigenotoxic and cogenotoxic agents. Toxicology, 2004, 198(1-3), 329-340.

[31] Patlolla, A. K.; Barnes, C.; Hackett, D.; Tchounwou, P. B., Potassium Dichromate Induced Cytotoxicity, Genotoxicity and Oxidative Stress in Human Liver Carcinoma (HepG(2)) Cells. International Journal of Environmental Research and Public Health, 2009, 6(2), 643-653.

[32] He, X.; Lin, G. X.; Chen, M. G.; Zhang, J. X.; Ma, Q., Protection against chromium (VI)-induced oxidative stress and apoptosis by Nrf2. Recruiting Nrf2 
into the nucleus and disrupting the nuclear Nrf2/Keap1 association. Toxicological Sciences, 2007, 98(1), 298-309.

[33] Renzulli, C.; Galvano, F.; Pierdomenico, L.; Speroni, E.; Guerra, M. C., Effects of rosmarinic acid against aflatoxin B-1 and ochratoxin-A-induced cell damage in a human hepatoma cell line (Hep G2). Journal of Applied Toxicology, 2004, 24(4), 289-296.

[34] Lou, H.; Jing, X.; Ren, D.; Wei, X.; Zhang, X., Eriodictyol protects against H2O2induced neuron-like PC12 cell death through activation of Nrf2/ARE signaling pathway. Neurochemistry International, 2012, 61(2), 251-257. 


\section{Figure Captions}

Fig. 1 - Chromatographic profile at $280 \mathrm{~nm}$ of $M$. aquatica (gray line) and L. dentata (bold line) purified ethanolic extracts.

Fig. 2 - Viability of human hepatoblastoma HepG2 cells exposed for $72 \mathrm{~h}$ to increasing concentrations of $M$. aquatica and $L$. dentata extracts (1 to $200 \mu \mathrm{g} / \mathrm{mL})$. Values are means of percentage of cell viability with respect to control \pm S.E.M. from 3-4 independent experiments performed in triplicate.

Fig. 3 - Protective effect of M. aquatica and L. dentata purified ethanolic extracts (50 $\mu \mathrm{g} / \mathrm{mL}$ ) in ROS incremented production (A) or decrement of cell viability (B) of human hepatoblastoma HepG2 cells at basal ( $\square$, A and B) or under toxic conditions induced with potassium dichromate $(\mathrm{DK})$ at $5 \mu \mathrm{M}(\mathrm{A}, \mathbf{\square})$ and $25 \mu \mathrm{M}(\mathrm{A}, \mathbf{\square})$ for $48 \mathrm{~h}$, or with 1.5 of DK for $72 \mathrm{~h}(\mathrm{~B}, \boldsymbol{\square})$. Values are expressed as means \pm S.E.M. of percentage of ROS production compared to control, from 3-4 independent experiments performed in triplicate. Ma, M. aquatica purified ethanolic extract; Ld, L. dentata purified ethanolic extract. $* \mathrm{p}<0.05 ; * * * p<0.001$ when compared to cells exposed to 5,25 $\mu \mathrm{M}(\mathrm{A})$ or $1.5 \mu \mathrm{M}(\mathrm{B})$ potassium dichromate, in the absence of extract; ${ }^{\#} \mathrm{p}<0.05$; ${ }^{\# \#} \mathrm{p}<$ 0.001 when compared to untreated cells. 
Table 1- Test range, slope and intercept values of calibration curve, correlation coefficient, LOQs and LODs for standard compounds

\begin{tabular}{cccccccc}
\hline $\begin{array}{c}\text { Standard } \\
\text { Compound }\end{array}$ & $\begin{array}{c}\text { Range } \\
\text { concentration } \\
(\boldsymbol{\mu g} / \mathbf{m L})\end{array}$ & $\mathbf{n}^{\mathbf{a}}$ & $\begin{array}{c}\text { Slope }^{\mathbf{b}}(\mathbf{a r e a} \\
\text { counts/mg) }\end{array}$ & $\begin{array}{c}\text { Intercept }^{\mathbf{b}} \\
(\mathbf{a r e a} \text { counts } \\
/ \mathbf{m g})\end{array}$ & $\mathbf{R}^{\mathbf{2}}$ & $\begin{array}{c}\text { LOD } \\
(\boldsymbol{\mu g} / \mathbf{m L})\end{array}$ & $\begin{array}{c}\text { LOQ } \\
(\boldsymbol{\mu g} / \mathbf{m L})\end{array}$ \\
\hline E-7O-G & $10-136$ & 5 & $1106( \pm 10) \times 10^{4}$ & $34( \pm 8) \times 10^{3}$ & 0.9995 & 5.6 & 16.9 \\
\hline N-7O-G & $5-68$ & 5 & $136( \pm 1) \times 10^{5}$ & $2( \pm 4) \times 10^{3}$ & 0.9991 & 2.7 & 8.1 \\
\hline L-7O-G & $45-473$ & 5 & $385( \pm 10) \times 10^{4}$ & $7( \pm 2) \times 10^{4}$ & 0.9945 & 40.6 & 123.2 \\
\hline RA & $15-173$ & 5 & $143( \pm 1) \times 10^{5}$ & $-10( \pm 1) \times 10^{4}$ & 0.9992 & 6.4 & 19.3
\end{tabular}

E-7O-G, eriodictyol-7-O-glucoside; N-7O-G, naringenin-7-O-glucoside; L-7O-G, luteolin-7-O-glucoside; RA, rosmarinic acid.

${ }^{a}$ Number of points used for the regression of standard solutions. Injections were done in triplicate.

${ }^{\mathrm{b}}$ The standard deviation in the slope and intercept of the regression line is shown in parenthesis 
Table 2 - Identification of the phenolic compounds in HPLC eluting peaks of $M$. aquatica and L. dentata purified ethanolic extracts through HPLC-DAD and ESI-MS combined analysis.

\begin{tabular}{|c|c|c|c|c|c|}
\hline Peak & $\begin{array}{c}\text { RT } \\
(\mathbf{m i n})\end{array}$ & $\lambda_{\max }$ & {$[\mathbf{M}-\mathbf{H}]^{-}$} & $\begin{array}{l}\text { Main fragment } \\
\text { ESI }^{-} \text {MS }^{\mathbf{n}}\end{array}$ & Compound \\
\hline \multicolumn{6}{|c|}{ M. aquatica } \\
\hline 1 & 15.0 & 283,325 & 595 & $\begin{array}{l}\mathrm{MS}^{2} \text { [595]: 287; } \mathrm{MS}^{3}: \text { [287]: 269(3\%), 151(100\%), } \\
\text { 125(2\%), 107(1\%); } \mathrm{MS}^{4} \text { [151]: } 107\end{array}$ & Eriodictyol- $O$-rutinoside \\
\hline \multirow{3}{*}{2} & \multirow{3}{*}{16.8} & \multirow{3}{*}{$254,267,345$} & $\begin{array}{c}593 \\
(100 \%)\end{array}$ & $\begin{array}{l}\mathrm{MS}^{2} \text { [593]: } 285(100 \%), 267(10 \%), 241(3 \%) ; \mathrm{MS}^{3}: \\
\text { [285]: } 241(100 \%), 217(67 \%), 199(65 \%), 175(62 \%), \\
\text { 151(20\%) }\end{array}$ & Luteolin-7-O-rutinoside \\
\hline & & & $\begin{array}{c}609 \\
(35 \%)\end{array}$ & $\begin{array}{l}\mathrm{MS}^{2}[609]: 343(5 \%), 301(100 \%), 255(2 \%), 271 \\
(2 \%), 179(2 \%) ; \mathrm{MS}^{3}[301]: 273(10 \%), 257(10 \%) \\
179(100 \%), 151(60 \%)\end{array}$ & Rutin \\
\hline & & & $\begin{array}{c}449 \\
(40 \%)\end{array}$ & $\mathrm{MS}^{2}$ [449]: 287; $\mathrm{MS}^{3}$ [287]: 151 & Eriodictyol-7-O-glucoside \\
\hline \multirow{3}{*}{3} & \multirow{3}{*}{17.9} & \multirow[t]{2}{*}{$254,267,350$} & $\begin{array}{c}447 \\
(15 \%)\end{array}$ & $\begin{array}{l}\mathrm{MS}^{2} \text { [447]: 285; } \mathrm{MS}^{3} \text { [285]: 243(50\%), 241(100\%), } \\
217(90 \%), 201(7 \%), 199(85 \%), 175(75 \%)\end{array}$ & Luteolin-7-O-glucoside \\
\hline & & & $\begin{array}{c}461 \\
(40 \%)\end{array}$ & $\mathrm{MS}^{2}$ [461]: 285 & Luteolin-7- $O$ - glucuronide \\
\hline & & 282,333 & $\begin{array}{c}579 \\
(100 \%)\end{array}$ & $\mathrm{MS}^{2}$ [579]: 271; $\mathrm{MS}^{3}:[271]: 177(5 \%), 151(100 \%)$ & Naringenin-7- $O$-rutinoside \\
\hline \multirow{3}{*}{4} & \multirow{3}{*}{19.5} & 266,336 & $\begin{array}{c}577 \\
(50 \%)\end{array}$ & $\begin{array}{l}\mathrm{MS}^{2} \text { [577]: 269; } \mathrm{MS}^{3}: \text { [269]: 241(10\%), 225(100\%), } \\
\text { 224(60\%), 203(30\%), 183(10\%), 182(20\%), 151(35\%) }\end{array}$ & Apigenin-7-O-rutinoside \\
\hline & & 283,325 & $\begin{array}{c}609 \\
(100 \%)\end{array}$ & $\begin{array}{l}\mathrm{MS}^{2} \text { [609]: 301(100), 286(<1), 242(<1); } \mathrm{MS}^{3}[301]: \\
\text { 286(100\%), 283(40\%), 257(25\%), 242(40\%), } \\
\text { 233(3\%), 199(5\%), 125(10\%); } \mathrm{MS}^{4}[286]: 268(5 \%), \\
\text { 258(75\%), 242(100\%), 199(5\%), 174(5\%); } \mathrm{MS}^{5}[241]: \\
\text { 227(100\%), 199(60\%) }\end{array}$ & Hesperetin-7-O-rutinoside \\
\hline & & $253,267,345$ & $\begin{array}{c}461 \\
(40 \%)\end{array}$ & $\begin{array}{l}\mathrm{MS}^{2} \text { [461]: 285; } \mathrm{MS}^{3} \text { [285]: 243(20\%), 241(90\%), } \\
217(45 \%), 199(10 \%), 175(100 \%), 133(14 \%)\end{array}$ & Luteolin-7-O-glucuronide \\
\hline 5 & 21.1 & 290,328 & $\begin{array}{c}359 \\
(100 \%)\end{array}$ & $\begin{array}{l}\mathrm{MS}^{2} \text { [359]: 315(2\%), 223(10\%), 197(15\%), 179(20\%), } \\
\text { 161(100\%), 133(1\%); } \mathrm{MS}^{3}[179]: 135\end{array}$ & Rosmarinic acid \\
\hline \multicolumn{6}{|c|}{ L. dentata } \\
\hline 3 & 18.1 & $253,267,345$ & 461 & $\begin{array}{l}\mathrm{MS}^{2}[461]: 285 ; \mathrm{MS}^{3} \text { [285]: 267(10\%),257(15\%), } \\
\text { 243(55\%), 241(100\%), 217(45\%), 199(45\%), } \\
\text { 197(8\%), 175(55\%), 151(10\%) }\end{array}$ & Luteolin-7-O-glucuronide \\
\hline \multirow{2}{*}{5} & \multirow{2}{*}{21.1} & 266,329 & $\begin{array}{c}431 \\
(10 \%)\end{array}$ & $\begin{array}{l}\mathrm{MS}^{2} \text { [431]: 269; } \mathrm{MS}^{3} \text { [269]: 241(5\%), 227(15\%), } \\
225(100 \%), 201(13 \%), 197(15 \%), 183(15 \%), \\
\text { 181(10\%), 151(7\%), 149(15\%),117(5\%) }\end{array}$ & Apigenin-7-O-glucoside \\
\hline & & 290,328 & $\begin{array}{c}359 \\
(100 \%)\end{array}$ & $\begin{array}{l}\mathrm{MS}^{2} \text { [359]: 223(15\%), 197(25\%), 179(30\%), } \\
\text { 161(100\%), 133(3\%); } \mathrm{MS}^{3} \text { [179]: 161(20\%), } \\
\text { 135(100\%) }\end{array}$ & Rosmarinic acid \\
\hline 6 & 26.3 & 266,330 & 473 & $\mathrm{MS}^{2}$ [473]: 413(1\%), 269(100\%) & $\begin{array}{l}\text { Apigenin-7-O-(6" } \\
\text { acetyl)glucoside }\end{array}$ \\
\hline
\end{tabular}


Table 3 - Quantification at $280 \mathrm{~nm}$ of the main phenolic constituents of M. aquatica and $L$. dentata purified ethanolic extracts

\begin{tabular}{|c|c|c|c|c|c|}
\hline \multirow[b]{2}{*}{ Peak } & \multirow[b]{2}{*}{$\begin{array}{l}\text { Quantified } \\
\text { with }\end{array}$} & \multicolumn{2}{|c|}{ M. aquatica } & \multicolumn{2}{|c|}{ L. dentata } \\
\hline & & Compound & $\begin{array}{l}\mathrm{mg} / \mathrm{g} \text { of } \\
\text { extract }^{\mathrm{a}}\end{array}$ & Compound & $\begin{array}{l}\mathrm{mg} / \mathrm{g} \text { of } \\
\text { extract }^{\mathrm{a}}\end{array}$ \\
\hline 1 & E-7O-G & $\begin{array}{l}\text { Eriodictyol-7-O- } \\
\text { rutinoside }\end{array}$ & $\begin{array}{l}144.6 \pm 6.2 \\
\quad(48 \%)\end{array}$ & & \\
\hline 2 & L-7O-G & $\begin{array}{l}\text { Luteolin-7-O- } \\
\text { rutinoside }\end{array}$ & $\begin{array}{l}43.3 \pm 2.8 \\
(14 \%)\end{array}$ & & \\
\hline 3 & $\begin{array}{l}\text { N-7O-G/ } \\
\text { L-7O-G }\end{array}$ & $\begin{array}{l}\text { Naringenin-7-O- } \\
\text { rutinoside }\end{array}$ & $\begin{array}{l}24.4 \pm 1.0 \\
(8 \%)\end{array}$ & $\begin{array}{l}\text { Luteolin-7-O- } \\
\text { glucuronide }\end{array}$ & $\begin{array}{l}26.2 \pm 2.0 \\
\quad(28 \%)\end{array}$ \\
\hline 4 & E-7O-G & $\begin{array}{l}\text { Hesperitin-7-O- } \\
\text { rutinoside }\end{array}$ & $\begin{array}{l}25.9 \pm 1.0 \\
\quad(9 \%)\end{array}$ & & \\
\hline 5 & RA & Rosmarinic acid & $\begin{array}{l}64.2 \pm 2.4 \\
\quad(21 \%)\end{array}$ & Rosmarinic acid & $\begin{array}{l}67.8 \pm 3.3 \\
(72 \%)\end{array}$ \\
\hline \multirow[t]{2}{*}{6} & & & & $\begin{array}{l}\text { Apigenin-7-O-(acetyl) } \\
\text { glucoside }\end{array}$ & * \\
\hline & & Total & $302.5 \pm 8.0$ & & $93.9 \pm 2.0$ \\
\hline
\end{tabular}

E-7O-G, eriodictyol-7-O-glucoside; L-7O-G, luteolin-7-O-glucoside; N-7O-G, naringenin-7-O-glucoside; RA, rosmarinic acid

Mean values \pm SEM

${ }^{\mathrm{a}}$ Values in parenthesis are expressed as the percentage of total quantified phenolic compounds

* Below limit of quantification 
Figure 1

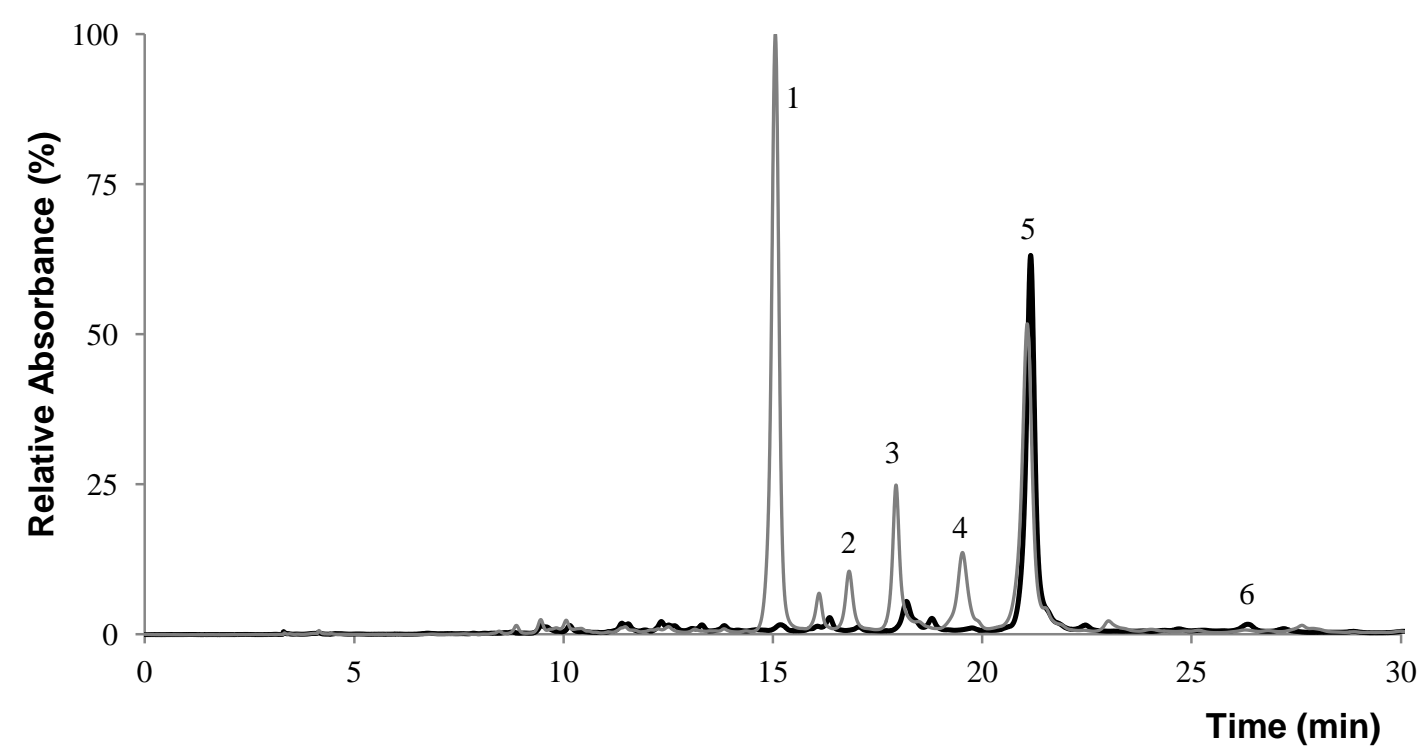


Figure 2

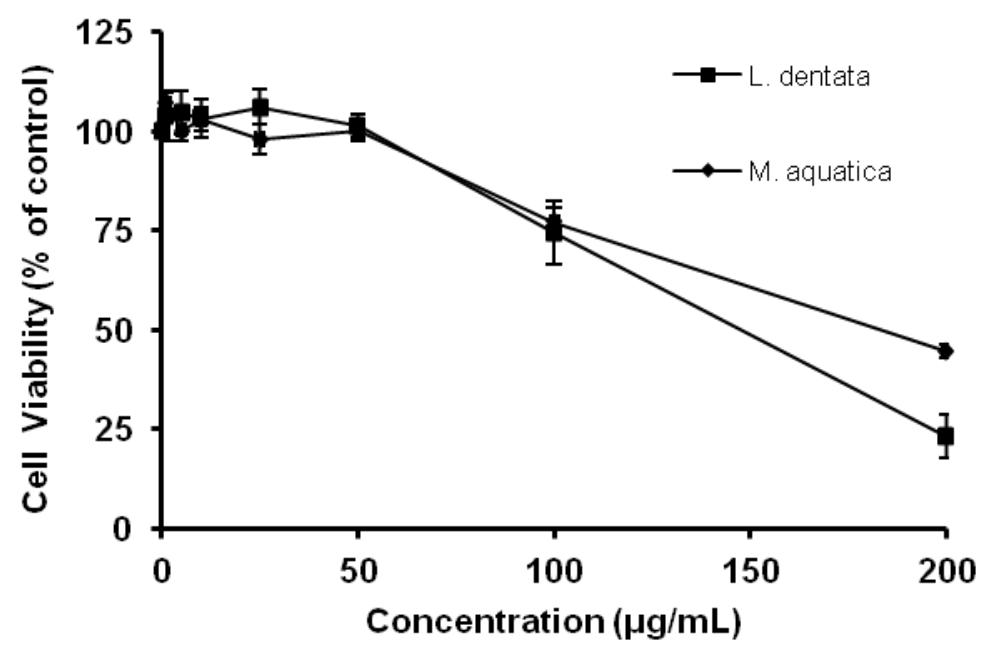


Figure 3

A

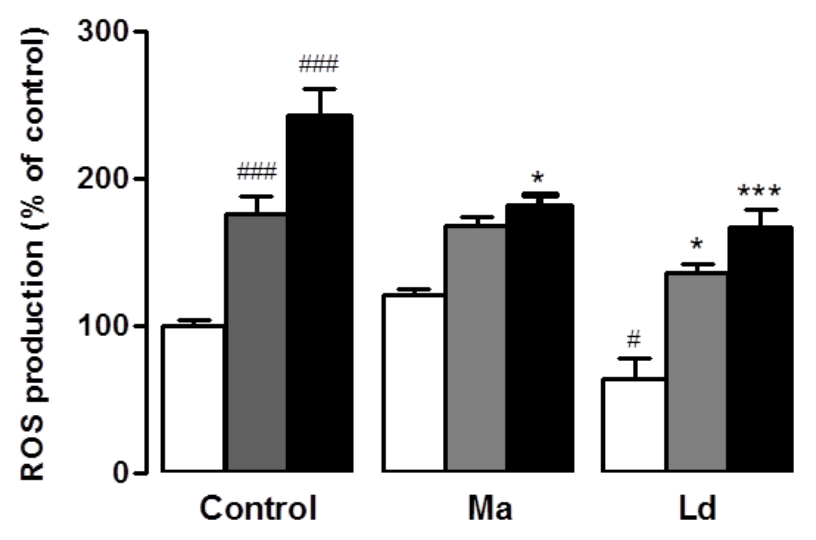

B

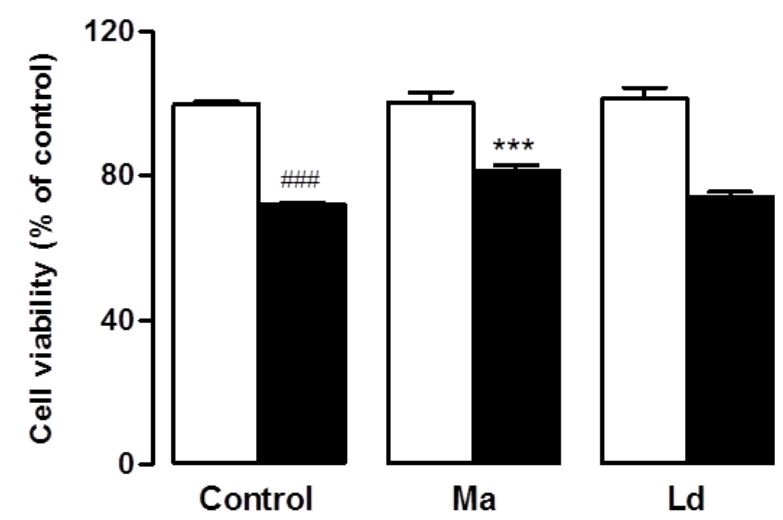




\subsection{Protective effects of phenolic constituents from Cytisus MULTIFLORUS, LAMIUM ALBUM L. AND THYMUS $X$ CITRIODORUS ON LIVER CELLS}

Plants are widely used in traditional medicine due to their beneficial activities. From the large diversity of the plant constituents, special relevance has been given to their polyphenolic compounds, which are often able to counteract oxidative stress, through various mechanisms. In this study, the potential antioxidant and cytoprotective effects of Cytisus multiflorus, Lamium album L. and Thymus $x$ citriodorus, as well as those exhibited by their phenolic constituents, were evaluated by in vitro assays on liver cells. The extracts were shown to have high antioxidant effects in the two chemical tests with a potency order of $L$. album $>T$. $x$ citriodorus $>C$. multiflorus. The cellular-based assays indicated that all extracts can counteract the increased ROS production induced by potassium dichromate. The high capacities in counteracting ROS formation in oxidative stress conditions in HepG2 cells were shown to be correlated with the ROS-scavenging activities of the polyphenols present in the extracts. The cells treatment with $L$. album and $C$. multiflorus extracts also induced an hepatoprotective effect of 34 or $24 \%$ ( $6 \mathrm{~h}$ of incubation with DK $200 \mu \mathrm{M}$ ) and 11 or $12 \%$ (72h of incubation with DK $2 \mu \mathrm{M}$ ), respectively. The cytoprotective effect of $L$. album purified ethanolic extract seems related to the presence of verbascoside, which exhibited the highest cytoprotective action between the tested reference compounds. Overall, these results suggest that $C$. multiflorus and $L$. album purified ethanolic extracts are good antioxidants and that polyphenols present in their extracts play an important role in the beneficial properties of these plants.

The results obtained in this section of the Doctoral Thesis have been used to write the manuscript entitled "Protective effects of phenolic constituents from Cytisus multiflorus, Lamium album L. and Thymus citriodorus on liver cells" which is in press in Journal of Functional Foods. 


\title{
Protective effects of phenolic constituents from Cytisus multiflorus, Lamium album L. and Thymus citriodorus on liver cells
}

\author{
Olívia R. Pereira ${ }^{a, b}$, Rocio I.R. Macias ${ }^{c}$, María J. Perez ${ }^{c, d}$, Jose J.G. Marin ${ }^{c, *}$, \\ Susana M. Cardoso ${ }^{b, e, *}$ \\ ${ }^{a}$ Departamento de Tecnologias de Diagnóstico e Terapêutica, Escola Superior de Saúde, Instituto Politécnico de Bragança, Av. D. Afonso V, \\ 5300-121 Bragança, Portugal \\ ${ }^{\mathrm{b}}$ CIMO, Escola Superior Agrária, Instituto Politécnico de Bragança, Campus de Santa Apolónia, 5301-855 Bragança, Portugal \\ ${ }^{\mathrm{C} L a b o r a t o r y}$ of Experimental Hepatology and Drug Targeting (HEVEFARM), Biomedical Research Institute of Salamanca (IBSAL), CIBERehd, \\ University of Salamanca, Spain \\ ${ }^{d}$ Hospital Universitario de Salamanca, Instituto de Estudios de Ciencias de la Salud de Castilla y León, Salamanca, Spain \\ ${ }^{e}$ CERNAS, Escola Superior Agrária, Instituto Politécnico de Coimbra, Bencanta, 3040-316 Coimbra, Portugal
}

\section{A R T I C L E I N F O}

Article history:

Received 28 January 2013

Received in revised form

15 March 2013

Accepted 20 March 2013

Available online $\mathrm{xxxx}$

\section{Keywords:}

Cytisus multiflorus

Lamium album L.

Thymus citriodorus

Phenolic compounds

Cytoprotection

Antioxidant activity

\begin{abstract}
A B S T R A C T
The present study investigated the antioxidant and cytoprotective effects of purified ethanolic extracts of Cytisus multiflorus, Lamium album L. and Thymus citriodorus plants. These extracts showed high antioxidant activity in DPPH and reducing power assays. Using a model of chemical stress induced by potassium dichromate (DK) in human hepatoblastoma HepG2 cells, $50 \mu \mathrm{g} / \mathrm{mL}$ of C. multiflorus, L. album and T. citriodorus extracts decreased the rate of reactive oxygen species (ROS) production by $35 \%, 26 \%$ and $20 \%$, respectively, when exposed to $25 \mu \mathrm{M}$ of DK. This effect was also observed for the treatment of cells with individual polyphenolic compounds determined in the extracts, or with mixtures prepared with individual polyphenolic compounds simulating the phenolic composition of the extracts. Additionally, the purified ethanolic extracts and the prepared polyphenolic mixtures showed a cytoprotective effect against DK-induced toxicity. The overall results emphasize the contribution of polyphenols in antioxidant and cytoprotective properties of the studied plants.
\end{abstract}

(ㄷ) 2013 Elsevier Ltd. All rights reserved.

\section{Introduction}

Reactive oxygen species (ROS) are generated in a variety of intracellular processes and in particular in the mitochondrial electron transport chain, where the redox complexes donate electrons to oxygen leading to the formation of $\mathrm{O}_{2}^{--}$, a precursor of the majority of ROS. The imbalance between cellular antioxidant defenses and the overproduction of ROS leads to oxidative stress. This biological condition is closely associated to aging processes and to several diseases, including cardiovascular, neurodegenerative, inflammatory diseases and cancer. In particular in liver, ROS excess can induce cell damage in lipids, proteins and DNA, inducing necrosis and apoptosis of hepatocytes, amplifying the inflammatory response

* Corresponding authors. Addresses: Department of Physiology and Pharmacology, IBSAL, Campus Miguel de Unamuno EID S-09, 37007 Salamanca, Spain. Fax: +34 923294669 (J.J.G. Marin). CERNAS, Escola Superior Agrária, Instituto Politécnico de Coimbra, Bencanta, 3040316 Coimbra, Portugal. Tel.: +351 239 802940; fax: +351 273239802979 (S.M. Cardoso)

E-mail addresses: jjgmarin@usal.es (J.J.G. Marin), scardoso@esac.pt (S.M. Cardoso).

1756-4646/\$ - see front matter @ 2013 Elsevier Ltd. All rights reserved.

http://dx.doi.org/10.1016/j.jff.2013.03.014 
and initiating hepatic fibrosis (Sanchez-Valle, Chavez-Tapia, Uribe, \& Mendez-Sanchez, 2012).

On the other hand, plants are frequently used in traditional medicine due to their beneficial activities. Among the plant constituents, special relevance has been given to their polyphenolic compounds (PPCs), which often exhibit high antioxidant capacity and hence, are able to counteract oxidative stress (Deng et al., 2013). Polyphenols can act as antioxidants through various mechanisms, including hydrogendonating reactions, metal chelation, inhibition of cytochrome P450 isoforms and up-regulation or protection of antioxidant defenses (e.g. intracellular glutathione levels) (Krishnaiah, Sarbatly, \& Nithyanandam, 2011). The potential antioxidant effect of herbs, or that of isolated phenolic compounds, has been extensively determined by in vitro tests. Common methods for measuring the antioxidant capacity include free radical 2,2-diphenyl-1-picrylhydrazyl (DPPH) scavenging, $\mathrm{OH}$ radical scavenging ability, reducing/antioxidant power (FRAP), oxygen radical absorbance capacity (ORAC), and Trolox equivalence antioxidant capacity (TEAC), among others (Hossain, Patras, Barry-Ryan, Martin-Diana, \& Brunton, 2011; Jabri-Karoui, Bettaieb, Msaada, Hammami, \& Marzouk, 2012). Despite their usefulness, these assays have some limitations, the most important being the difficulty of extrapolating results to the in vivo conditions, whereas closer results can be obtained by the use of cultured cells. In this context, the HepG2 cell line, derived from human hepatoblastoma, has been extensively used as an in vitro model to investigate the beneficial potency of plant extracts with respect to hepatic injury conditions. Although these are tumour cells, they retain many of the specialized functions of normal human hepatocytes (including some which are lost by primary hepatocytes) and hence are considered as a valid tool for this type of studies (Chen, Ma, Liang, Peng, \& Zuo, 2011; Hanlon, Robbins, Hammon, \& Barnes, 2009; Wang, Lee, Chen, Yu, \& Duh, 2012). Toxicity in these cells can be induced by several agents including hydrogen peroxide, tert-butyl hydroperoxide, aflatoxin B1 and potassium dichromate (DK) (Mersch-Sundermann, Knasmuller, Wu, Darroudi, \& Kassie, 2004). The latter enters rapidly into the cells resulting in oxidative damage by means of ROS generation, lipid peroxidation, DNA breakdown and induction of apoptosis (Son et al., 2010).

Cytisus multiflorus (L'Hér.) Sweet, Lamium album L. and Thymus citriodorus are Mediterranean plants which are used, either raw or cooked, for confection of distinct local dishes. Moreover, these plants are frequently consumed in the form of tea, or in food supplements preparations (L. album L.), due to their claimed medicinal properties. Concretely, C. multiflorus has been used because of its diuretic, anti-inflammatory, anti-hypertensor and antidiabetic properties (Gião et al., 2007) while lemon thyme (T. citriodorus) has been used due to its deodorant, antiseptic and antimicrobial activities, as well as in the treatment of asthma and other respiratory diseases (Omidbaigi, Sefidkon, \& Hejazi, 2005). In turn, L. album is famous due to its antioxidant, antispasmodic, mucolytic, diuretic, haemostatic, anti-inflammatory and anticancer activities (Paduch, Wójciak-Kosior, \& Matysik, 2007).

The phenolic composition of these plants has been studied and, according to that, $C$. multiflorus is rich in chrysin deriva- tives (e.g. chrysin-7-O- $\beta$-D-glucopyranoside), also containing other flavones and flavonol hexoside derivatives (Pereira, Silva, Domingues, \& Cardoso, 2012a). L. album extracts mainly include the phenylpropanoid glycosides verbascoside and isoverbascoside, and some phenolic acids and flavonoids (Paduch et al., 2007; Pereira, Domingues, Silva, \& Cardoso, 2012b), while phenolic extracts of $\mathrm{T}$. citriodorus are rich in O-glycosidic derivatives of luteolin and apigenin, as well as in rosmarinic acid (Pereira, Domingues, \& Cardoso, 2010).

The antioxidant properties of $C$. multiflorus (Barros, Cabrita, Boas, Carvalho, \& Ferreira, 2011) and L. album extracts (Armatu, Colceru-Mihul, Bubueanu, Draghici, \& Pirvu, 2010) have previously been evaluated by simple chemical methods (e.g. DPPH and ABTS assays) and through assessment of lipids oxidative damage. However, there are no similar assays focusing T. citriodorus extracts. Moreover, there is no available information on the ROS scavenging ability, as well as on the potential cytoprotective properties in cultured cells of C. multiflorus, $L$. album, and T. citriodorus polar extracts. This issue was investigated in the present work using a model of chemical stress induced in HepG2 cells by incubation with DK. The role of the main phenolic components of the three plant extracts on the ROS scavenging and on cytoprotective properties was also investigated in this experimental model.

\section{Experimental}

\subsection{Chemicals}

Porcine trypsin was purchased from Roche (Barcelona, Spain). Tripan blue, dimethyl sulphoxide (DMSO), "Minimum Essential Medium Eagle (MEM)" and RPMI-1640 culture media, mix of antibiotics and antimycotic, sodium piruvate, sodium bicarbonate, 3-(4,5-dimethylthiazol-2-yl)-2,5diphenyltetrazolium bromide (MTT), dichlorofluoresceindiacetate (DCFH-DA), cisplatin and DK were purchased from Sigma-Aldrich (Madrid, Spain). BHA (butylated hydroxyanisole) and DPPH radical (2,2-diphenyl-2-picrylhydrazyl) were obtained from Sigma Chemical Co. (St. Louis, MO, USA). Fetal bovine serum (FBS) was obtained from T.D.I. (Madrid, Spain). The phenolic standard compounds apigenin, chrysin, eriodictyol, quercetin, luteolin, naringenin, rosmarinic acid and verbascoside were obtained from Extrasynthese (Genay Cedex, France). Ascorbic acid was purchased from Panreac (Barcelona, Spain).

\subsection{Plant extracts}

C. multiflorus (flowers), L. album and T. citriodorus (aerial parts of both plants) were purchased from Ervital (Castro de Aire, Portugal) and the ethanolic purified extracts of the three plants were obtained and previously characterized regarding their phenolic components (Pereira et al., 2010, 2012a, $2012 b)$. The content of the main phenolics is summarized in Table 1 (in terms of aglycones and/or their derivatives) due to their relevance for the understanding of the present study. According to those studies, the total phenolic compounds in C. multiflorus, L. album and T. citriodorus purified ethanolic extracts accounted for $41 \%, 50 \%$ and $14.9 \%$ of its 
Table 1 - Mean content of phenolic compounds in Cytisus multiflorus, Lamium album and Thymus citriodorus extracts.

\begin{tabular}{|c|c|c|c|}
\hline Plant & Compound & $\begin{array}{l}\text { Mean content } \\
\text { (mg/g extract) }\end{array}$ & References \\
\hline \multirow[t]{4}{*}{ Cytisus multiflorus } & Chrysin plus derivatives & 72.8 & Pereira et al. (2012a) \\
\hline & Luteolin derivatives & 23.4 & \\
\hline & Apigenin plus derivatives & 20.0 & \\
\hline & Quercetin derivatives & 14.1 & \\
\hline \multirow[t]{4}{*}{ Lamium album } & Verbascoside plus derivatives & 272.9 & Pereira et al. (2012b) \\
\hline & Naringenin derivatives & 32.6 & \\
\hline & Apigenin derivatives & 32.3 & \\
\hline & Luteolin derivatives & 29.7 & \\
\hline \multirow[t]{5}{*}{ Thymus citriodorus } & Luteolin derivatives & 15.2 & Pereira et al. (2010) \\
\hline & Rosmarinic acid plus derivative & 12.7 & \\
\hline & Apigenin derivatives & 9.0 & \\
\hline & Eriodictyol derivatives & 5.7 & \\
\hline & Naringenin derivatives & 1.8 & \\
\hline
\end{tabular}

weight, respectively. Moreover, the phenolic composition of C. multiflorus was mainly composed of chrysin-7-O- $\beta-D-$ glucopyranoside and of considerable amounts of a dihydroxyflavone isomer of chrysin. These two derivatives plus chrysin accounted for $56 \%$ of the total phenolic compounds quantified in the extract that is equivalent to $72.8 \mu \mathrm{g} / \mathrm{mg}$ extract. Moreover, luteolin derivatives (2"-O-pentosyl-6-C-hexosyl-luteolin, 2"-O-pentosyl-8-C-hexosyl-luteolin and orientin) accounted for approximately $23.4 \mu \mathrm{g} / \mathrm{mg}$ extract, while apigenin plus its derivatives and quercetin derivatives accounted for 20.0 and $14.1 \mu \mathrm{g} / \mathrm{mg}$ extract, respectively. In turn, the amount of verbascoside and its derivatives in the L. album purified ethanolic extract represented approximately $56 \%$ of the total phenolic content $(272.9 \mu \mathrm{g} / \mathrm{mg}$ extract). Remaining phenolic compounds in the extract enclosed isoscutellarein glycosides and 7-0-derivatives of naringenin, apigenin and luteolin, with the three latter accounting for $32.6,32.3$ and $29.7 \mu \mathrm{g} / \mathrm{mg}$ extract, respectively. In turn, $T$. citriodorus purified ethanolic extract mainly comprised luteolin derivatives $(15.2 \mu \mathrm{g} / \mathrm{mg}$ extract) and rosmarinic acid and derivative $(12.7 \mu \mathrm{g} / \mathrm{mg}$ extract), besides minor amounts of eriodictyol and apigenin derivatives.

\subsection{DPPH test}

The scavenging capacity of each purified ethanolic extract was carried out by DPPH radical test following the Kirby and Schmidt (1997) procedure with adaptations. For that, distinct methanolic test solutions $(0.05,0.1,0.25,0.5$ and $0.8 \mathrm{mg} / \mathrm{mL})$ of the extracts of $C$. multiflorus, $L$. album and T. citriodorus were prepared and $0.1 \mathrm{~mL}$ of each solution was added to $1.7 \mathrm{~mL}$ of a methanolic solution of DPPH $(60 \mu \mathrm{M})$ in a test tube, followed by vigorous shaken. After $30 \mathrm{~min}$ of incubation in the dark, the absorbance of the mixtures was measured in a spectrophotometer at $517 \mathrm{~nm}$, against a blank (absence of DPPH). The radical scavenging activity of each purified ethanolic extract was calculated as the percentage of DPPH discoloration, using the equation of Yen and Duh (1994):

$\%$ DPPH radical scavenging $=\left[\left(A_{C}(0)-A_{E}(t)\right) / A_{C}(0)\right]^{*} 100$, where: $A_{C}(0)=$ Absorbance of the control at $t=0 \mathrm{~min} ; A_{E}$ $(t)=$ Absorbance of the extract at $t=30 \mathrm{~min}$.
Based on graphic values of percentage of DPPH inhibition us. extract concentration, the $\mathrm{EC}_{50}$ (concentration of the extract able to inhibit the $50 \%$ of the DPPH radical) of each extract was estimated. Ascorbic acid was used as positive control.

\section{4. $\quad$ Reducing power test}

The ability of C. multiflorus, L. album and T. citriodorus extracts in reducing iron (III) was assessed by the method described by Barros et al. (2011), performed in a 48-well plate using an ELX800 Microplate Reader (BioTek Instruments, Inc., Winooski, VT, USA). For that, $0.5 \mathrm{~mL}$ of distinct concentrations $(0.05,0.067,0.1,0.125,0.25 \mathrm{mg} / \mathrm{mL})$ of each extract of interest was mixed with $0.5 \mathrm{~mL}$ of phosphate buffer $(0.2 \mathrm{M}, \mathrm{pH} 6.6)$ and $0.5 \mathrm{~mL}$ of a $1 \%$ potassium hexacyanoferrate $\left[\mathrm{K}_{3} \mathrm{Fe}(\mathrm{CN})_{6}\right]$ aqueous solution. After $20 \mathrm{~min}$ of incubation at $50{ }^{\circ} \mathrm{C}, 0.5 \mathrm{~mL}$ of $10 \%$ trichloroacetic acid was added and $0.8 \mathrm{~mL}$ of the mixture was poured in the 48 -wells with $0.8 \mathrm{~mL}$ of deionized water and $0.16 \mathrm{~mL}$ of $\mathrm{FeCl}_{3}(0.1 \%, \mathrm{w} / \mathrm{v})$. The absorbance was measured at $690 \mathrm{~nm}$. The mean absorbance values were plotted against concentration, a linear regression analysis was carried out, and the $\mathrm{EC}_{50}$ value, corresponding to the extract concentration providing 0.5 of absorbance, was determined. BHA was used as positive control.

\subsection{Cell culture}

Human hepatoblastoma HepG2 cells (HB-8065) were obtained from the American Type Culture Collection (Manassas, VA, USA) and cultured in polystyrene flasks (Falcon) with MEM supplemented with $1 \mathrm{mM}$ of sodium pyruvate, $26.2 \mu \mathrm{M}$ of sodium bicarbonate, inactivated FBS $10 \%(\mathrm{v} / \mathrm{v})$ and $1 \%$ of a mixture antibiotic-antimycotic solution [penicillin $(20 \mathrm{U} / \mathrm{mL})$, streptomycin $(0.02 \mathrm{mg} / \mathrm{mL})$ and amphotericin B $(0.05 \mu \mathrm{g} / \mathrm{mL})$ ] under an atmosphere of $5 \% \mathrm{CO}_{2}$ at $37^{\circ} \mathrm{C}$. Cells were plated onto 96 well-plates at a density of $0.3 \times 10^{6}$ or $0.15 \times 10^{6}$ cells/mL ( 6 or $72 \mathrm{~h}$ treatments, respectively) in a total volume of $100 \mu \mathrm{L}$, for the MTT experiments. Alternatively, cells were seeded in 6 well-plates at a density of $0.1 \times 10^{6}$ cells $/ \mathrm{mL}$, in a total volume of $2 \mathrm{~mL}$, for ROS experiments. 


\subsection{Preparation of test solutions for cellular assays}

The C. multiflorus, L. album and T. citriodorus purified ethanolic extracts were dissolved in culture medium at a concentration of $1 \mathrm{mg} / \mathrm{mL}$ and sterilized by UV light exposure ( $1 \mathrm{~h}, 30 \mathrm{~W})$, in order to avoid the contamination of the cultured cells. Phenolics stability under these conditions was confirmed by HPLCDAD analysis (data not shown). Standard compounds, namely apigenin, chrysin, eriodictyol, quercetin, luteolin, naringenin, rosmarinic acid and verbascoside, were dissolved in sterile dimethyl sulphoxide (DMSO) $(50 \mathrm{mg} / \mathrm{mL})$. These concentrated solutions were subsequently diluted in culture medium to obtain final concentrations of $1-200 \mu \mathrm{g} / \mathrm{mL}$ for purified ethanolic extracts or $50 \mu \mathrm{g} / \mathrm{mL}$ for standard compounds. The final DMSO concentration was lower than $0.5 \%$ and did not affect the cell viability or ROS production (data not shown). Based on the phenolic composition of each target purified ethanolic extract (Pereira et al., 2010, 2012a, 2012b), three mixtures of phenolic standards were also prepared taking into account the amount of the individual PPCs determined in $50 \mu \mathrm{g} / \mathrm{mL}$ of each extract. In this sense, luteolin, apigenin, quercetin and chrysin were used with final individual concentrations of $1.2,1.0,0.7,3.6 \mu \mathrm{g} / \mathrm{mL}$, respectively, for the C. multiflorus mixture. These quantities corresponded to the global amount of each aglycone (plus their derivatives) in the extract. In a similar way, the L. album PPCs mixture was prepared with the phenolic compounds verbascoside, luteolin, apigenin, naringenin with concentrations of $14.0,1.4,1.6,1.6 \mu \mathrm{g} / \mathrm{mL}$, respectively, while that of $\mathrm{T}$. citriodorus was obtained with $0.3,0.8,0.1,0.5,0.6 \mu \mathrm{g} / \mathrm{mL}$ of eriodictyol, luteolin, naringenin, apigenin and rosmarinic acid, respectively.

\subsection{Determination of cell viability by MTT assay}

Twenty-four hours after seeding the cells, the culture medium was replaced by fresh medium containing the desired concentration of agents (extracts, PPCs mixtures or individual standards) in the presence or absence of DK. After incubation for 6 or $72 \mathrm{~h}$, viability of HepG2 cells was determined by the formazan formation from tetrazolium salt (MTT) by living cells (Briz, Serrano, Macias, \& Marin, 2000). Briefly, cells were rinsed with $\mathrm{PBS}$ and incubated with $0.5 \mathrm{mg} / \mathrm{mL}$ of MTT dissolved in RPMI medium for $4 \mathrm{~h}$ at $37^{\circ} \mathrm{C}$. Cell lysis and dissolution of purple formazan crystals were accomplished by adding $100 \mu \mathrm{L}$ of SDS and further incubation overnight at $37^{\circ} \mathrm{C}$. The absorbance was read at $595 \mathrm{~nm}$ in an ELISA reader (model 550, Bio-Rad, Madrid, Spain). Cisplatin $(0.3-30 \mu \mathrm{g} / \mathrm{mL}$ ), a classic cytotoxic compound, was used as a positive control of toxicity. Cell viability was calculated as the percentage of living cells compared to untreated (control) cells. Moreover, possible unspecific reactions between MTT and the antioxidants were rejected by a control experiment performed in the presence of MTT and distinct extract/standard concentrations, in the absence of cells.

The short-term exposure $(6 \mathrm{~h})$ was used to determine acute cell toxicity while long-term exposure $(72 \mathrm{~h})$ permitted to calculate the antiproliferative effect in accordance to preestablished methods (Zakaria et al., 2011). Toxicity in those experiments were, respectively, induced by DK at 200 and $2 \mu \mathrm{M}$, since previous studies by our group have shown a de- crease on the HepG2 cells viability of about $25-45 \%$ in those conditions (data not shown).

\subsection{Determination of ROS production by flow cytometry}

After $48 \mathrm{~h}$ incubation of HepG2 cells with the desired concentration of agents and/or DK, the medium was replaced by RPMI medium containing $5 \mu \mathrm{g} / \mathrm{mL}$ of the probe DCFH-DA (a stable non-fluorescent cell permeable compound). After 30 min, cells were trypsinized and resuspended in FBS freemedium. When internalized by the cell, DCFH-DA is hydrolyzed to DCFH by intracellular esterases and rapidly oxidized to the highly green fluorescent compound 2,7-dichlorofluorescein (DCF) by endogenous ROS, in particular hydroperoxides. ROS generation was measured and analyzed in a FACSCalibur flow cytometer (BD Biosciences, San Jose, CA, USA) and CellQuest software (BD Biosciences). The values were expressed as percentage of ROS formation by untreated cells. Note that the $48 \mathrm{~h}$ of exposure was used to investigate the effect on ROS production before the end-point used for the cell growth inhibition assays. Increment in ROS production by approximately two and three fold was accomplished by their treatment with $\mathrm{DK}$ at 5 and $25 \mu \mathrm{M}$, respectively.

\subsection{Statistical analysis}

Data were expressed as mean \pm S.E.M. of the number of experiments as indicated in the figure legends. The comparison between groups was performed by one-way ANOVA, followed by Dunnett's post hoc test.

\section{Results and discussion}

\subsection{Determination of the non-toxic concentration ranges of the purified extracts}

As mentioned above, the HepG2 cells are a well-known in vitro model for the assessment of protective activities of natural extracts or compounds in toxicological investigations in liver cells (Chen et al., 2011; Wang et al., 2012) and were herein used in the present study to evaluate the potential antioxidant and cytoprotective effects of C. multiflorus, L. album, and $\mathrm{T}$. citriodorus purified ethanolic extracts.

As a first approach, HepG2 cells were treated with different concentrations (1-200 $\mu \mathrm{g} / \mathrm{mL}$ ) of purified ethanolic extracts, in order to determine the non-toxic range of doses, allowing to choose the appropriate concentrations of extracts to be used in the following experiments. This was evaluated by means of the MTT assay, an extensively used test to monitor cell survival. Our results demonstrated that the toxicity of the three extracts was very low compared to that of cisplatin, a classic cytotoxic compound. Except for T. citriodorus, the cell viability measured after $72 \mathrm{~h}$ of treatment was unaffected up to $200 \mu \mathrm{g} /$ $\mathrm{mL}$ (Fig. 1). Based on this study, the $50 \mu \mathrm{g} / \mathrm{mL}$ dose was selected for testing the ROS scavenging and cytoprotective activities of the three extracts, while that of $200 \mu \mathrm{g} / \mathrm{mL}$ was additionally selected for C. multiflorus and L. album.

Regarding the MTT assay, it is also important to note that despite some of the main aglycones from the plant extracts 


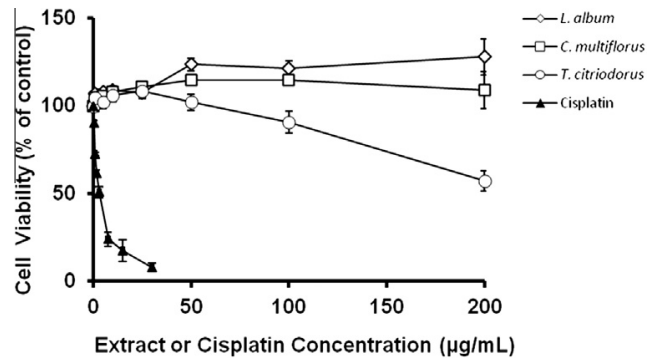

Fig. 1 - Viability of human hepatoblastoma HepG2 cells incubated with increasing concentrations of the purified ethanolic extracts from Cytisus multiflorus, Lamium album and Thymus citriodorus (1-200 $\mu \mathrm{g} / \mathrm{mL})$ for $72 \mathrm{~h}$. Cisplatin was used as a positive control of cytotoxicity in HepG2 cells. Values are means of percentage of cell viability with respect to control \pm S.E.M. from four independent experiments performed in triplicate.

have been shown to exert cytotoxic effects on HepG2 cells, e.g. chrysin, verbascoside and luteolin (Ahmed, Mohamed, El-Dib, \& Hamed, 2009; Yee et al., 2003), no toxicity was registered for the investigated phenolic enriched ethanolic extracts. Different experimental conditions (e.g. treatment period), presence of different forms of phenolic derivatives (aglycones or glycosides) in extracts or counterbalanced effects of the multiple extract components could account for this result. Besides this, it should be highlighted that the slight increment in cell viability observed after incubation with the low doses of extracts is not surprising and has been observed with other molecules with antioxidant properties, such as bile acids, at non-toxic doses (Briz et al., 2000).

\subsection{Antioxidant and cytoprotective properties of purified ethanolic extracts and PPCs}

\subsubsection{Chemical models}

The antioxidant potential of purified ethanolic extracts was first estimated by the DPPH radical scavenging and reducing power assays. These two chemical tests are widespread used for estimating the antioxidant capacity of plant extracts related to their ability to trap the DPPH radical, and to reduce $\mathrm{Fe}^{3+}$ to $\mathrm{Fe}^{2+}$, respectively. As observed in Table 2, the three purified ethanolic extracts had close DPPH EC $\mathrm{E}_{50}$ values, rang- ing from approximately 11 to $13 \mu \mathrm{g} / \mathrm{mL}$. Considering that these $\mathrm{EC}_{50}$ values are only five times lower than that obtained for ascorbic acid (Table 2), we might conclude that the purified ethanolic extracts of the three plants have a considerable DPPH scavenging ability. The DPPH scavenging ability has previously been described for C. multiflorus and for L. album phenolic extracts, while to the best of our knowledge this topic has not yet been addressed for T. citriodorus. In general, the DPPH EC $\mathrm{C}_{50}$ herein estimated were lower than those previously described, which is probably due to the extract purification step applied in the present study. Previous described DPPH $\mathrm{EC}_{50}$ values ranged from 71.5 to $2000 \mu \mathrm{g} / \mathrm{mL}$ (Barros et al., 2011; Luis, Domingues, \& Duarte, 2011) and from 30 to $466 \mu \mathrm{g} / \mathrm{mL}$ (Armatu et al., 2010; Valyova, Dimitrova, Ganeva, Mihova Kapchina-Toteva, \& Petkova Yordanova, 2011), for polar extracts of C. multiflorus and L. album, respectively.

Regarding the reducing power assay, the $\mathrm{EC}_{50}$ values obtained in the present study for C. multiflorus, L. album and T. citriodorus purified ethanolic extracts were respectively $95.7 \pm 2.7,67.9 \pm 5.0$ and $88.2 \pm 0.8 \mu \mathrm{g} / \mathrm{mL}$ (or 1.6, 2.3, $1.9 \mathrm{mmol}$ $\mathrm{BHA} / \mathrm{g}$ extract, respectively). Similarly to the DPPH assay data, the present $\mathrm{EC}_{50}$ values for $C$. multiflorus are much inferior to those previously reported $(410 \mu \mathrm{g} / \mathrm{mL})$ by other group (Barros et al., 2011). These results indicate a 2 to 3-fold less general capacity for reducing $\mathrm{Fe}^{3+}$ to $\mathrm{Fe}^{2+}$ than that of $\mathrm{BHA}$, the potent synthetic antioxidant used as positive control (Table 2). The reducing capacity order was L. album $>$ T. citriodorus $>C$. multiflorus.

\subsubsection{Protective effects against ROS production and decrease} in cell viability induced by potassium dichromate in human hepatoblastoma HepG2 cells

The antioxidant capacity of the three purified ethanolic extracts was further evaluated for their ROS scavenging abilities, on the potassium dichromate-stimulated human hepatoblastoma HepG2 cell model. As observed in Fig. 2, the exposure of the cells to 5 or $25 \mu \mathrm{M}$ potassium dichromate caused a significant increase in the intracellular ROS levels, of 1.9-fold and 2.9-fold of the control, respectively. However, co-incubation of cells with potassium dichromate plus the target purified ethanolic extracts partially prevented the increase in intracellular ROS levels. This effect was dosedependent for C. multiflorus and L. album purified ethanolic extracts (Fig. 2A and B). Note that in contrast to the results ob-

Table 2 - Radical scavenging potential and reducing power of Cytisus multiflorus, Lamium album and Thymus citriodorus extract plants.

\begin{tabular}{|c|c|c|}
\hline \multirow[t]{2}{*}{ Compound/plant extract } & \multicolumn{2}{|c|}{$\mathrm{EC}_{50}(\mu \mathrm{g} / \mathrm{mL})$} \\
\hline & Radical scavenging ${ }^{\mathrm{A}}$ & Reducing power ${ }^{B}$ \\
\hline Ascorbic acid & $2.5 \pm 0.2^{\mathrm{a}}$ & - \\
\hline BHA & - & $27.1 \pm 0.6^{c}$ \\
\hline Cytisus multiflorus & $13.4 \pm 0.5^{\mathrm{b}}$ & $95.7 \pm 2.7^{\mathrm{d}}$ \\
\hline Lamium album & $11.2 \pm 0.5^{b}$ & $67.9 \pm 5.0^{\mathrm{e}}$ \\
\hline Thymus citriodorus & $11.7 \pm 1.5^{\mathrm{b}}$ & $88.2 \pm 0.8^{\mathrm{d}}$ \\
\hline
\end{tabular}



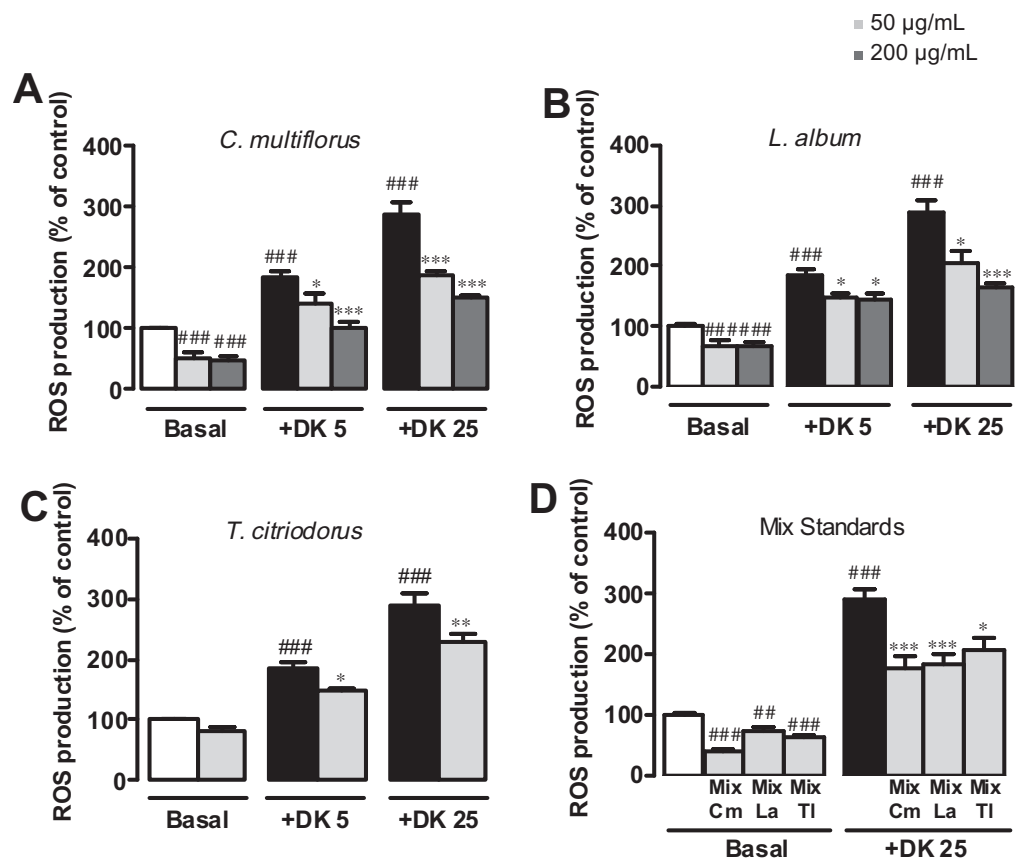

Fig. 2 - Protective effect of Cytisus multiflorus (A), Lamium album (B) and Thymus citriodorus (C) purified ethanolic extracts and mixtures of PPCs simulating each plant extract (D) on intracellular ROS production in human hepatoblastoma HepG2 cells induced with potassium dichromate (DK). Cells were incubated in the absence $(\square, \square)$ or presence of two non-toxic extract concentrations: at $50 \mu \mathrm{g} / \mathrm{mL}$ () (for all the extracts) or at $200 \mu \mathrm{g} / \mathrm{mL}()$ for Cytisus multiflorus and Lamium album extracts and with mixtures of PPCs that simulate each plant extract $(D$,$) . With the exception of the basal condition, the cells were$ exposed to DK at 5 or $25 \mu \mathrm{M}$, for $48 \mathrm{~h}$. The white columns ( $\square$ ) represent the control condition and the black columns ( $\square$ ) represent the incubation of HepG2 cells with DK alone. Values are expressed as means \pm S.E.M. of percentage of ROS production versus control, from 3-4 independent experiments performed in triplicate. Mix Cm, Cytisus multiflorus PPCs mixture; Mix La, Lamium album PPCs mixture; Mix Tc, Thymus citriodorus PPCs mixture. Statistical analysis was performed by one-way ANOVA, followed by Dunnett's post hoc test. ${ }^{*} p<0.05 ;{ }^{* *} p<0.01 ;{ }^{* * *} p<0.001$ when compared to cells exposed to 5 or

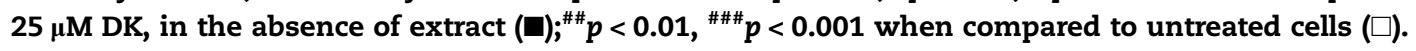

tained in the chemical models, C. multiflorus purified ethanolic extract was the most effective in counteracting the potassium dichromate-increased ROS formation. In more detail, treatment of cells with C. multiflorus, L. album or T. citriodorus extracts at $50 \mu \mathrm{g} / \mathrm{mL}$ decreased the intracellular ROS formation by about $19 \%, 23 \%$ and $21 \%$, respectively $(5 \mu \mathrm{M}$ potassium dichromate-stimulated cells) or about $35 \%, 26 \%$ and $20 \%$, respectively $(25 \mu \mathrm{M}$ potassium dichromate-stimulated cells) (Fig. 2A-C). Moreover, for both potassium dichromate treatment conditions, purified ethanolic extract from C. multiflorus $>$ L. album (both at $200 \mu \mathrm{g} / \mathrm{mL}$ ) decreased ROS production. These extracts also reduced the intracellular ROS levels at basal conditions, e.g., in the absence of potassium dichromate; C. multiflorus (47-53\%), L. album ( $\approx 30 \%)$ and $\mathrm{T}$. citriodorus $(\approx 15 \%$, not significant).

Overall, the results obtained in this part of the work suggested that the three purified ethanolic extracts, and in particular those of C. multiflorus and L. album can act as good ROS scavenging agents in oxidative stress conditions in hepatic cells. To the best of our knowledge, this is the first report describing the potential ROS-scavenging ability of these purified ethanolic extracts.

The purified ethanolic extracts also exhibited protection against the potassium dichromate-induced acute toxicity $(200 \mu \mathrm{M}, 6 \mathrm{~h})$ or long-term toxicity $(2 \mu \mathrm{M}, 72 \mathrm{~h})$ as measured by the MTT assay. As can be observed in Fig. 3A, under acute toxic conditions, all the purified ethanolic extracts exerted a significant protection against the cell viability decrement (about 30\%). Moreover, the C. multiflorus and L. album extracts partially prevented cell viability decrement under long-term toxic conditions (Fig. 3B). The cytoprotective effects of the individual purified ethanolic extracts $(50 \mu \mathrm{g} / \mathrm{mL})$ were not potentiated by the treatment of the cells with combinations of two extracts $(25 \mu \mathrm{g} / \mathrm{mL}$ each), suggesting the absence of synergisms on the mentioned beneficial properties of the extracts.

In order to determine the role of PPCs in the observed protective effects of the three purified ethanolic extracts, the previous assays were performed with three PPCs mixtures prepared as described in the methods section by mixing the individual PPCs apigenin, chysin, eriodictyol, luteolin, naringenin, quercetin, rosmarinic acid and verbascoside to simulate the content determined in $50 \mu \mathrm{g} / \mathrm{mL}$ of C. multiflorus, L. album and T. citriodorus purified ethanolic extracts. It should be mentioned that aglycones were used instead of the glycosylated forms detected in these purified ethanolic extracts due to their commercial availability. Although some differences can be expected in the ROS scavenging capacity and in the cytoprotective activity between these two forms when tested under in vitro conditions, it is important to note that PPCs are 
A
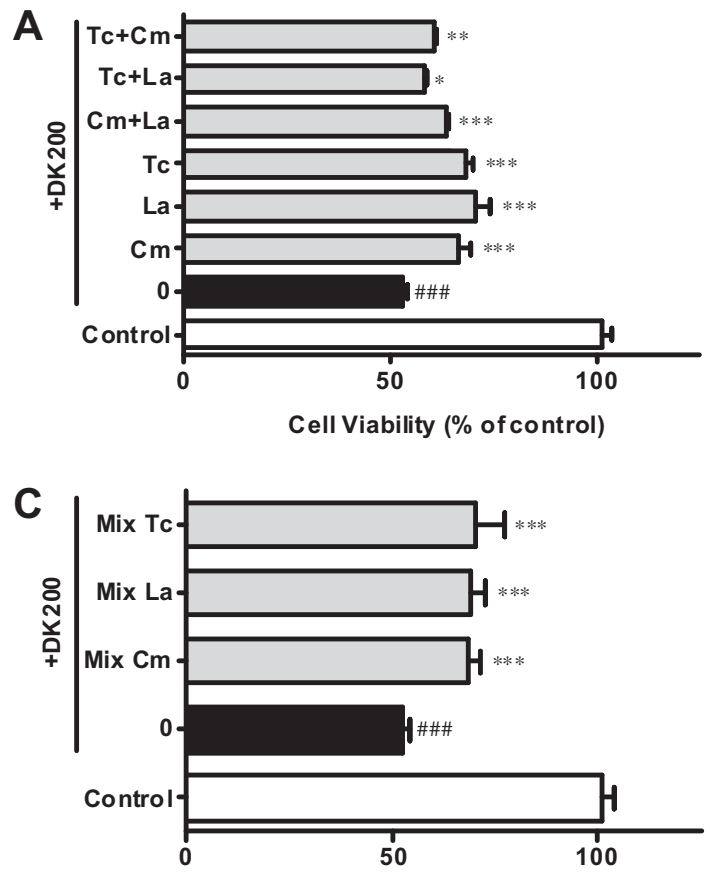

Cell Viability (\% of con trol)

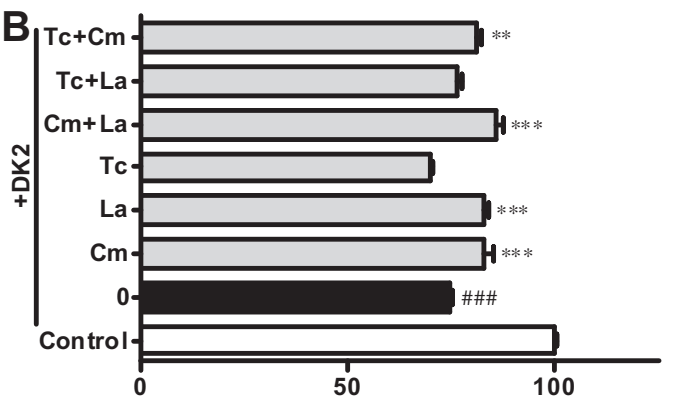

Cell Viability (\% of control)

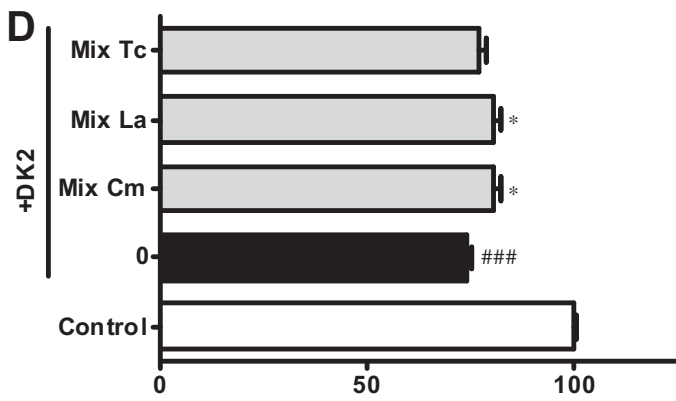

Cell Viability (\% of control)

Fig. 3 - Protective effect of Cytisus multiflorus (A), Lamium album (B) and Thymus citriodorus (C) purified ethanolic extracts and mixtures of PPCs simulating each plant extract (D) in the potassium dichromate (DK)-induced cell viability decrement of human hepatoblastoma HepG2 cells. Cells were incubated in the absence $(\square, \square)$ or presence of each extract (50 $\mu \mathrm{g} / \mathrm{mL})$ or their mixtures ( $25 \mu \mathrm{g} / \mathrm{mL}$ each) (, A, B) or alternatively with mixtures of PPCs that simulate each plant extract (, C, D). With the exception of the control condition $(\square)$ the cells were then exposed to potassium dichromate (DK) $200 \mu \mathrm{M}$ for $6 \mathrm{~h}$ (A, C) or $2 \mu \mathrm{M}$ for $72 \mathrm{~h}(B, D)$. The black columns $(\square)$ represent the incubation of HepG2 cells with DK alone. Values are means \pm S.E.M. of percentage of cell viability versus control, from 3-4 independent experiments performed at least in triplicate. Cm, Cytisus multiflorus extract; Mix Cm, Cytisus multiflorus PPCs mixture; La, Lamium album extract; Mix La, Lamium album PPCs mixture; Tc, Thymus citriodorus extract; Mix Tc, Thymus citriodorus PPCs mixture. Statistical analysis was performed by one-way ANOVA, followed by Dunnett's post hoc test. " $p<0.05 ;{ }^{* *} p<0.01,{ }^{* * *} p<0.001$ when compared to cells exposed to DK ( $\square$ ), in the absence of extract; ${ }^{\# \#} p<0.001$ when compared to untreated cells ( $\square$ ).

mainly absorbed in vivo as aglycones, since most classes of glycosylated PPCs are hydrolyzed in the intestine before absorption (D'Archivio et al., 2010).

As shown in Fig. 2D, a significant reduction of intracellular ROS production was observed both in basal conditions (mainly for the C. multiflorus mixture) and under co-treatment of cells with $25 \mu \mathrm{M}$ potassium dichromate and each PPCs mixture.

A similar result was observed in the MTT assay (Fig. 3C and D). The three PPCs mixtures prevented the cell viability reduction induced by short-term potassium dichromate exposure ( $\approx 32 \%$ ) (Fig. 3C), while the protection was lost after long-term incubation for the T. citriodorus mixture (Fig. 3D).

Literature data focusing on plant phenolics frequently associate their content to the health benefits, in particular with the antioxidant capacity of the extracts. This theory is valid, at least partially, in the present study. In fact, L. album extract, which is the most enriched in phenolics $(501 \mathrm{mg} / \mathrm{g})$, showed high antioxidant potential both in chemical models and in ROS scavenging and cytoprotective actions in potassium dichromate-exposed HepG2 cells. In turn, T. citriodorus extract, the most poor extract in phenolic compounds $(149 \mathrm{mg} / \mathrm{g})$ also presented the weakest antioxidant capacity in all the tested models and was not able to protect HepG2 cells from potassium dichromate toxicity for long incubation time (72 h).

This is the first study focusing on the potential antioxidant abilities and cytoprotective activities of C. multiflorus, L. album and T. citriodorus purified ethanolic extracts, as well as on the association of these beneficial effects to the main phenolic constituents of the extracts. The herein focused benefits have also been scarcely studied for plants of the three genera (Cytisus, Lamium and Thymus). To our knowledge, the antioxidant and cytoprotective effects of Cytisus scoparius plant were demonstrated by in vivo studies. Oral administration of the extract counteracted the decrease of superoxide dismutase and catalase and the increase of lipid peroxidation in a chronic unpredictable mild stress model in rats, and protected the liver from carbon tetrachloride-induced oxidative stress in rats by increasing the levels of glutathione and several antioxidant hepatic enzymes (Raja et al., 2007a, 2007b). Some of these effects have showed a good correlation with total phenolic content in the C. scoparius plant extract (Luis, Domingues, Gil, \& Duarte, 2009). Other in vivo work revealed important cytoprotective activities of Thymus vulgaris on an alcohol abuse model by reversing the reduction of the antioxidant capacity and 

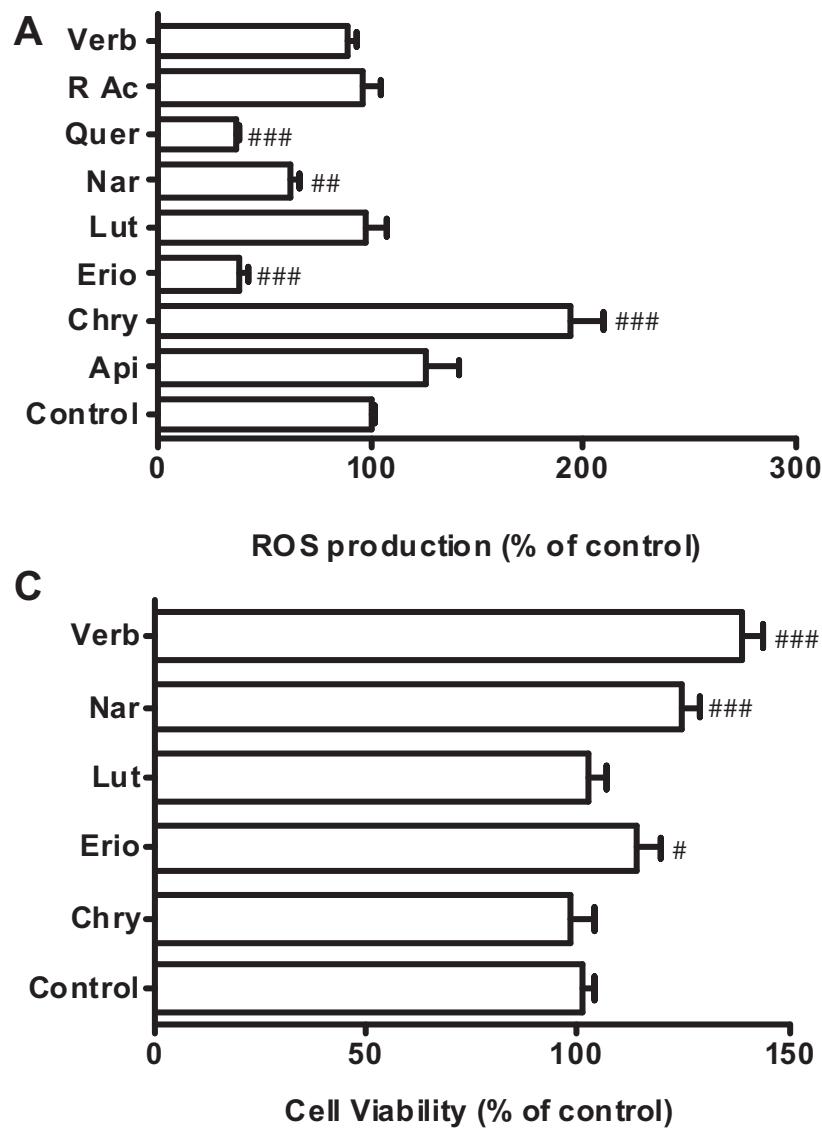

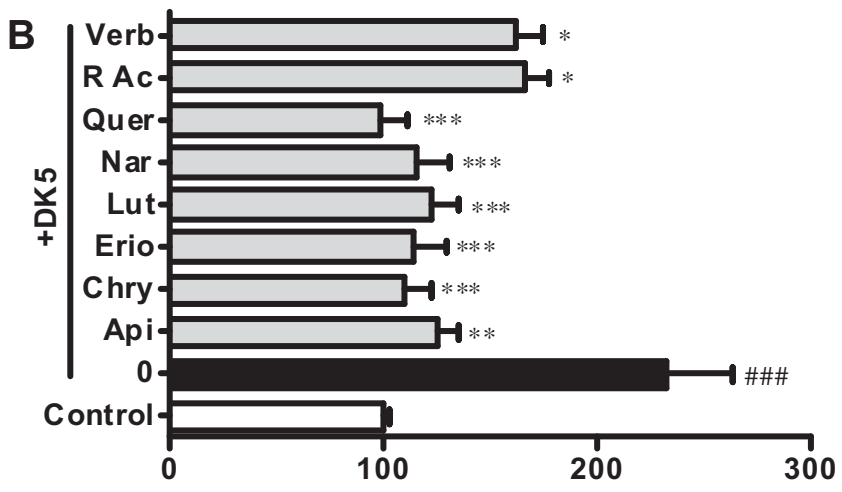

ROS production (\% of control)

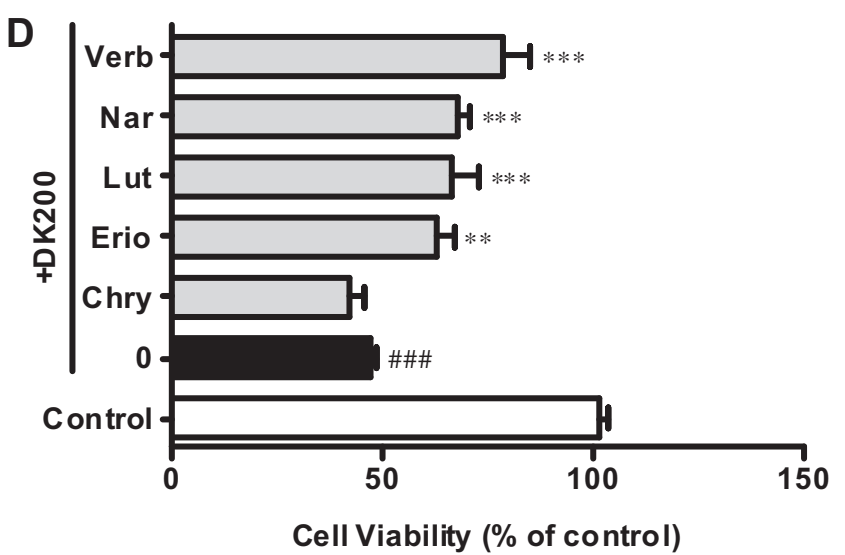

Fig. 4 - Protective effect of the individual standard compounds $(50 \mu \mathrm{g} / \mathrm{mL})$ on intracellular ROS production (A, B) or cells viability (C, D) of human hepatoblastoma HepG2 cells at basal (A and C) ( $\square$ ) or under toxic conditions (B, D) induced with potassium dichromate (DK) at $5 \mu \mathrm{M}$ for $48 \mathrm{~h}(\mathrm{~B}, \mathrm{D})$ or at $200 \mu \mathrm{M}$ for $6 \mathrm{~h}(\mathrm{D}$, )). The black columns ( $\square$ ) represented the incubation of HepG2 cells with DK alone. Values are means \pm S.E.M. of percentage of ROS production or cell viability versus control from 3-4 independent experiments performed at least in triplicate. Api, apigenin; Chry, chrysin; Eri, eriodictyol; Lut, luteolin; Nar, naringenin; Quer, quercetin; R Ac, rosmarinic acid; Ver, verbascoside. Statistical analysis was performed by one-way ANOVA, followed by Dunnett's post hoc test. ${ }^{*} p<0.05$; ${ }^{* *} p<0.01,{ }^{* * *} p<0.001$ when compared to cells exposed to DK ( $\left.\square\right)$ in the absence of individual standard compounds; ${ }^{\#} p<0.05 ;{ }^{\# \#} p<0.01 ;{ }^{\# \# \#} p 0.001$ when compared to untreated cells (control).

glutathione peroxidase in liver (Shati \& Elsaid, 2009). To our knowledge, liver bioprotection has not been previously exploited for Lamium plant extracts.

3.3. Relation between phytochemical content, antioxidant activities and cytoprotective effects

In order to determine the contribution of individual standard PPCs on the previously mentioned beneficial properties of the purified ethanolic extracts, ROS and MTT experiments were also performed for the majority of their individual phenolic constituents. As stated in the material and methods section, the C. multiflorus purified extract mainly contains chrysin derivatives and other flavones enclosing glycosidic derivatives of luteolin, apigenin and quercetin (Table 1). L. album purified ethanol extract is mainly composed of verbascoside, while other phenolics enclose derivatives of apigenin, luteolin and naringenin (Table 1) and major phenolic components in $\mathrm{T}$. citriodorus extract are luteolin-O-glucosides and rosmarinic acid, while it contained minor amounts of eriodictyol and apigenin derivatives (Table 1). Accordingly, apigenin, chrysin, eri- odictyol, quercetin, luteolin, naringenin, rosmarinic acid and verbascoside (all at $50 \mu \mathrm{g} / \mathrm{mL}$ ) were assessed for protection both in basal and under potassium dichromate-induced toxic conditions. Some of these compounds had been previously tested in HepG2 cells and in HepG2/C3A (a clonal derivative of HepG2), showing no cytotoxicity after incubation with the same range of concentrations for $72 \mathrm{~h}$ (Liu, Flynn, Ferguson, Hoagland, \& Yu, 2011).

As can be observed in Fig. 4A, HepG2 cell exposure to quercetin, eriodictyol and naringenin in the absence of potassium dichromate decreased the basal intracellular ROS production by about $63 \%, 62 \%$ and $38 \%$, respectively. Moreover, under stress conditions, all target PPCs $(50 \mu \mathrm{g} / \mathrm{mL})$ showed high ability to scavenge HepG2 intracellular ROS (Fig. 4B). From all the PPCs tested, flavonoids were the most efficient standard compounds. Indeed, the 3- and 5-hydroxyl groups with a 4-oxo function in $\mathrm{A}$ and $\mathrm{C}$ rings of the flavonol quercetin and the C2-C3 double bond with a 4-oxo function in C ring, common to all of them, are crucial structural characteristics in determining the antioxidant properties of PPCs. Additionally, the presence of an ortho-dihydroxy (3', 4'-OH) structure on the 
B-ring (catechol group), present in quercetin and luteolin, are important to improve the antioxidant properties (Dai \& Mumper, 2010). Besides these two compounds, the flavanone eriodictyol also showed high ROS scavenging capacity (about $50 \%)$. This fact is in accordance with previous literature data reporting a powerful antioxidant potential of this compound in several cellular models, including neuronal cell cultures, monocytes and retinal pigment epithelial ARPE-19 cells (Cho et al., 2012). One curious finding is the increase of ROS production caused by chrysin (Fig. 4A), suggesting that at that concentration, this compound can induce some toxicity in human hepatoblastoma HepG2 cells, a phenomena described for other antioxidant compounds (Crispo et al., 2010). Despite this, under oxidative stress conditions, this flavone showed high ROS scavenging protection (approximately 53\% ROS reduction in comparison to the control) (Fig. 4B).

Taking into account the amount of the individual PPCs in the purified ethanolic extracts and also their individual capacity in decreasing ROS production under oxidative stress conditions (Fig. 4B), it is possible to suggest that chrysin $(56 \%$ of the total phenolics in C. multiflorus purified ethanolic extract) is the main responsible for its high ROS scavenging capacity. Still note that, despite being present in lower amounts in the C. multiflorus ethanolic extract, luteolin and apigenin also should positively contribute for its antioxidant activities, since they also have high ROS scavenging capacity. Importantly, these latter compounds plus naringenin must for sure be taken into account when considering the ROS scavenging ability of L. album purified ethanolic extract. Despite their minor abundance in the purified ethanolic extract in comparison to verbascoside (54\% total phenolics), they exhibited almost twice of its capacity for decreasing potassium dichromate-stimulated increment of ROS levels. In turn, luteolin, apigenin and eriodictyol are the compounds mainly associated to the ROS scavenging ability of the T. citriodorus ethanolic extract.

Cytoprotective effects of individual phenolics, as measured by the MTT test, were investigated for the most abundant PPCs in each purified ethanolic extract (chrysin, verbascoside and luteolin) and also in the flavanones naringenin and eriodictyol, due to their high ROS scavenging properties. As shown in Fig. 4C, in the absence of potassium dichromate, none of the target standard compounds induced a significant decrease on the cell MTT reducing ability, indicating that at the concentration of $50 \mu \mathrm{g} / \mathrm{mL}$ and for a period treatment of $6 \mathrm{~h}$, all compounds are safe for the HepG2 cells. In good agreement with these results, verbascoside, naringenin, luteolin and eriodictyol counteracted the decrease in cell reducing activity induced by potassium dichromate at $200 \mu \mathrm{M}$ (Fig. 4D) by $49 \%, 28 \%, 26 \%$ and $19 \%$, respectively. Indeed, this protective effect was not only observed for the chrysin treatment.

The herein obtained results also suggest that the cytoprotective effects of L. album and T. citriodorus ethanolic extracts are closely related to their major phenol compounds (verbascoside and luteolin, respectively), in opposition to that observed for the C. multiflorus ethanolic extract. Curiously, the mechanism of cytoprotection of verbascoside, the one exhibiting the highest cytoprotective action, is not totally related to its ROS scavenging action, since this was lower than that of the remaining PPCs (Fig. 4B). Hence, other mechanisms of protection should be investigated in the future for the L. album phenolic extract. Attending that potassium dichromateinduced cytotoxicity engages a cascade of cellular events, enclosing DNA breakdown and the induction of apoptosis through caspases activation (He, Lin, Chen, Zhang, \& Ma, 2007; Son et al., 2010), and that the inhibition of some of these events has been previously associated to verbascoside (Fu, Pang, \& Wong, 2008), these are potential mechanisms involved in the cytoprotective action of L. album phenolic extract.

\section{Conclusion}

C. multiflorus and L. album purified ethanolic extracts are good antioxidants. Their high capacities in counteracting ROS formation in oxidative stress conditions in HepG2 cells are in good agreement with the ROS-scavenging activities of the PPCs present in the extracts. The cytoprotective effect of L. album purified ethanolic extract seems related to the presence of verbascoside, which exhibited the highest cytoprotective action from all the PPCs tested. Since the cytoprotective effect seems to be related to a ROS-independent scavenging action, the mechanisms involved in the cytoprotective action of L. album extract should be investigated. Overall, our results suggest that PPCs play an important role in the beneficial properties of these plants.

\section{Conflict of interest statement}

The authors declare that there are no conflicts of interest.

\section{Acknowledgements}

The authors acknowledge the financial support provided by the FCT to CERNAS (project PEst-OE/AGR/UI0681/2011). Olivia R. Pereira was supported by a PhD grant (SFRH/PROTEC/49600/ 2009). This study was also supported in part by the Ministerio de Ciencia e Innovación (Grant SAF2010-15517); the Instituto de Salud Carlos III, FIS (Grant PI11/00337) and the Junta de Castilla y León (Grants SA023A11-2, SA070A11-2 and BIO/03/ SA23/11), Spain.

\section{R E F E R E N C E S}

Ahmed, W. S., Mohamed, M. A., El-Dib, R. A., \& Hamed, M. M. (2009). New triterpene saponins from Duranta repens Linn. and their cytotoxic activity. Molecules, 14, 1952-1965.

Armatu, A., Colceru-Mihul, S., Bubueanu, C., Draghici, E., \& Pirvu, L. (2010). Evaluation of antioxidant and free scavenging potential of some Lamiaceae species growing in Romania. Romanian Biotechnological Letters, 15, 5274-5280.

Barros, L., Cabrita, L., Boas, M. V., Carvalho, A. M., \& Ferreira, I. C. F. R. (2011). Chemical, biochemical and electrochemical assays to evaluate phytochemicals and antioxidant activity of wild plants. Food Chemistry, 127, 1600-1608.

Briz, O., Serrano, M. A., Macias, R. I. R., \& Marin, J. J. G. (2000). Overcoming cisplatin resistance in vitro by a free and 
liposome-encapsulated bile acid derivative: Bamet-R2. International Journal of Cancer, 88, 287-292.

Chen, Z.-Y., Ma, K. Y., Liang, Y., Peng, C., \& Zuo, Y. (2011). Role and classification of cholesterol-lowering functional foods. Journal of Functional Foods, 3, 61-69.

Cho, N., Choi, J. H., Yang, H., Jeong, E. J., Lee, K. Y., Kim, Y. C., \& Sung, S. H. (2012). Neuroprotective and anti-inflammatory effects of flavonoids isolated from Rhus verniciflua in neuronal HT22 and microglial BV2 cell lines. Food and Chemical Toxicology, 50, 1940-1945.

Crispo, J. A. G., Ansell, D. R., Piche, M., Eibl, J. K., Khaper, N., Ross, G. M., \& Tai, T. C. (2010). Protective effects of polyphenolic compounds on oxidative stress-induced cytotoxicity in PC12 cells. Canadian Journal of Physiology and Pharmacology, 88, 429-438.

Dai, J., \& Mumper, R. J. (2010). Plant phenolics: Extraction, analysis and their antioxidant and anticancer properties. Molecules, 15, 7313-7352.

D’Archivio, M., Filesi, C., Vari, R., Scazzocchio, B., \& Masella, R. (2010). Bioavailability of the polyphenols: Status and controversies. International Journal of Molecular Sciences, 11, 1321-1342.

Deng, G. F., Lin, X., Xu, X. R., Gao, L. L., Xie, J. F., \& Li, H. B. (2013). Antioxidant capacities and total phenolic contents of 56 vegetables. Journal of Functional Foods, 5, 260-266.

Fu, G., Pang, H., \& Wong, Y. H. (2008). Naturally occurring phenylethanoid glycosides: Potential leads for new therapeutics. Current Medicinal Chemistry, 15, 2592-2613.

Gião, M. S., Gonzalez-Sanjose, M. L., Rivero-Perez, M. D., Pereira, C. I., Pintado, M. E., \& Malcata, F. X. (2007). Infusions of Portuguese medicinal plants: Dependence of final antioxidant capacity and phenol content on extraction features. Journal of the Science of Food and Agriculture, 87, 2638-2647.

Hanlon, P. R., Robbins, M. G., Hammon, L. D., \& Barnes, D. M. (2009). Aqueous extract from the vegetative portion of Spanish black radish (Raphanus sativus L. var. niger) induces detoxification enzyme expression in HepG2 cells. Journal of Functional Foods, 1, 356-365.

He, X., Lin, G. X., Chen, M. G., Zhang, J. X., \& Ma, Q. (2007). Protection against chromium (VI)-induced oxidative stress and apoptosis by Nrf2. Recruiting Nrf2 into the nucleus and disrupting the nuclear Nrf2/Keap1 association. Toxicological Sciences, 98, 298-309.

Hossain, M. B., Patras, A., Barry-Ryan, C., Martin-Diana, A. B., \& Brunton, N. P. (2011). Application of principal component and hierarchical cluster analysis to classify different spices based on in vitro antioxidant activity and individual polyphenolic antioxidant compounds. Journal of Functional Foods, 3, 179-189.

Jabri-Karoui, I., Bettaieb, I., Msaada, K., Hammami, M., \& Marzouk, B. (2012). Research on the phenolic compounds and antioxidant activities of Tunisian Thymus capitatus. Journal of Functional Foods, 4, 661-669.

Kirby, A. J., \& Schmidt, R. J. (1997). The antioxidant activity of Chinese herbs for eczema and of placebo herbs. Journal of Ethnopharmacology, 56, 103-108.

Krishnaiah, D., Sarbatly, R., \& Nithyanandam, R. (2011). A review of the antioxidant potential of medicinal plant species. Food and Bioproducts Processing, 89, 217-233.

Liu, Y., Flynn, T. J., Ferguson, M. S., Hoagland, E. M., \& Yu, L. (2011). Effects of dietary phenolics and botanical extracts on hepatotoxicity-related endpoints in human and rat hepatoma cells and statistical models for prediction of hepatotoxicity. Food and Chemical Toxicology, 49, 1820-1827.

Luis, A., Domingues, F., \& Duarte, A. P. (2011). Bioactive compounds, RP-HPLC analysis of phenolics, and antioxidant activity of some Portuguese shrub species extracts. Natural Product Communications, 6, 1863-1872.
Luis, A., Domingues, F., Gil, C., \& Duarte, A. P. (2009). Antioxidant activity of extracts of Portuguese shrubs: Pterospartum tridentatum, Cytisus scoparius and Erica spp. Journal of Medicinal Plants Research, 3, 886-893.

Mersch-Sundermann, V., Knasmuller, S., Wu, X.-j., Darroudi, F., \& Kassie, F. (2004). Use of a human-derived liver cell line for the detection of cytoprotective, antigenotoxic and cogenotoxic agents. Toxicology, 198, 329-340.

Omidbaigi, R., Sefidkon, F., \& Hejazi, M. (2005). Essential oil composition of Thymus citriodorus L. cultivated in Iran. Flavour and Fragrance Journal, 20, 237-238.

Paduch, R., Wójciak-Kosior, M., \& Matysik, G. (2007). Investigation of biological activity of Lamii albi flos extracts. Journal of Ethnopharmacology, 110, 69-75.

Pereira, O. R., Domingues, M. R. M., \& Cardoso, S. M. (2010). Thymus citriodorus: Phenolic characterization and antioxidant activity. In Paper presented at the XXV The International Conference On Polyphenols.

Pereira, O. R., Domingues, M. R. M., Silva, A. M. S., \& Cardoso, S. M. (2012b). Phenolic constituents of Lamium album: Focus on isoscutellarein derivatives. Food Research International, 48, 330-335.

Pereira, O. R., Silva, A. M. S., Domingues, M. R. M., \& Cardoso, S. M. (2012a). Identification of phenolic constituents of Cytisus multiflorus. Food Chemistry, 131, 652-659.

Raja, S., Ahamed, H. N., Kumar, V., Mukherjee, K., Bandyopadhyay, A., \& Mukherjee, P. K. (2007a). Exploring the effect of Cytisus Scoparius on markers of oxidative stress in rats. Iranian Journal of Pharmacology \& Therapeutics, 6, 15-21.

Raja, S., Ahamed, K., Kumar, V., Mukherjee, K., Bandyopadhyay, A., \& Mukherjee, P. K. (2007b). Antioxidant effect of Cytisus scoparius against carbon tetrachloride treated liver injury in rats. Journal of Ethnopharmacology, 109, 41-47.

Sanchez-Valle, V., Chavez-Tapia, N. C., Uribe, M., \& MendezSanchez, N. (2012). Role of oxidative stress and molecular changes in liver fibrosis: A review. Current Medicinal Chemistry, $18,18$.

Shati, A. A., \& Elsaid, F. G. (2009). Effects of water extracts of thyme (Thymus vulgaris) and ginger (Zingiber officinale Roscoe) on alcohol abuse. Food and Chemical Toxicology, 47, 1945-1949.

Son, Y. O., Hitron, J. A., Wang, X., Chang, Q. S., Pan, J. J., Zhang, Z., Liu, J. K., Wang, S. X., Lee, J. C., \& Shi, X. L. (2010). Cr(VI) induces mitochondrial-mediated and caspase-dependent apoptosis through reactive oxygen species-mediated p53 activation in JB6 Cl41 cells. Toxicology and Applied Pharmacology, 245, 226-235.

Valyova, M. S., Dimitrova, M. A., Ganeva, Y. A., Mihova KapchinaToteva, V., \& Petkova Yordanova, Z. (2011). Evaluation of antioxidant and free radical scavenging potential of Lamium album L. growing in Bulgaria. Journal of Pharmacy Research, 4, 945-947.

Wang, B.-S., Lee, C. P., Chen, Z.-T., Yu, H. M., \& Duh, P.-D. (2012). Comparison of the hepatoprotective activity between cultured Cordyceps militaris and natural Cordyceps sinensis. Journal of Functional Foods, 4, 489-495.

Yee, S. B., Lee, J. H., Chung, H. Y., Im, K. S., Bae, S. J., Choi, J. S., \& Kim, N. D. (2003). Inhibitory effects of luteolin isolated from Ixeris sonchifolia Hance on the proliferation of HepG2 human hepatocellular carcinoma cells. Archives of Pharmacal Research, 26, 151-156.

Yen, G. C., \& Duh, P. D. (1994). Scavenging effect of methanolic extracts of peanut hulls on free-radical anda active-oxygen species. Journal of Agricultural and Food Chemistry, 42, 629-632.

Zakaria, Z. A., Rofiee, M. S., Teh, L. K., Salleh, M. Z., Sulaiman, M. R., \& Somchit, M. N. (2011). Bauhinia purpurea leaves' extracts exhibited in vitro antiproliferative and antioxidant activities. African Journal of Biotechnology, 10, 65-74. 


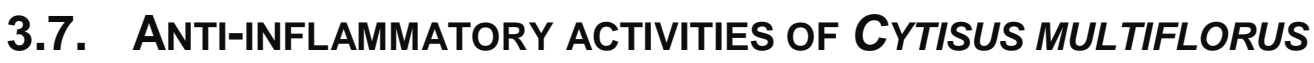

Historically, extracts and preparations of plants are the basis of traditional medicine and the starting point for the discovery of new therapeutic agents [1]. Cytisus multiflorus is a leguminous shrub native from Iberian Peninsula that is distributed in the south-west Mediterranean region. The plant is used in folk medicine and it is claimed to have various health benefits, including anti-inflammatory properties [2, 3]. Yet, the antiinflammatory usage of $C$. multiflorus is totally based on ethnopharmacological information, while no scientific data focusing this capacity, or its molecular targets of action, has been reported. Moreover, anti-inflammatory mechanisms in Cytisus genus have been scarcely reported and, to the author's knowledge, this property was only described for $C$. aeolicus, which was shown to inhibit leukotriene B4 production in rat polymorphonuclear leukocytes [4]. During inflammation, leukocytes, such as macrophages are recruited to the site of damage, which leads to a "respiratory burst" due to an increased uptake of oxygen and, thus, an increased release and accumulation of reactive species at the site of damage. On the other hand, inflammatory cells, such as macrophages, also produce soluble mediators, namely nitric oxide (NO) produced by inducible nitric oxide synthase (iNOS), leukotrienes formed by lipooxigenase (LOX) and prostaglandins produced by cyclooxygenase 2 (COX-2), which act by further recruiting inflammatory cells to the site of damage and producing more reactive species. This sustained inflammatory/oxidative environment leads to a vicious circle, which can damage healthy neighboring epithelial and stromal cells and over a long period of time may lead to chronic illnesses, namely diabetes, neurodegenerative and cardiovascular diseases. Since the overproduction of reactive species and pro-inflammatory mediators raises and maintains inflammation, compounds targeting their expression are good candidates for attenuating inflammatory diseases.

Once the antioxidant effect of $C$. multiflorus extract and of their phenolic compounds was demonstrated in chemical and cell based assays [5] and, as the antioxidant activity is usually well correlated with anti-inflammatory properties $[6,7]$, the latter were herein searched in order to clarify the possible anti-inflammatory mechanisms of $C$. multiflorus. Tests included the monitoring of scavenging activity of reactive species formed during the inflammatory response $\left(\mathrm{HOCl}, \mathrm{NO}{ }^{*}\right)$, as well the inhibitory ability of key pro-inflammatory enzymes, namely lipoxygenase (LOX), inducible nitric oxide synthase (iNOS) and cyclooxygenase-2 (COX-2) in an in vitro model of inflammation. 


\subsubsection{Materials and Methods}

\section{Chemicals}

Lipopolysaccharide (LPS) from E. coli (serotype 026:B6) was purchased from Sigma Chemical Co. (St. Louis, MO, USA). Dulbecco's Modified Eagle Medium ATCC and fetal serum were obtained from Gibco (Paisley, UK). The protease and phosphatase inhibitor cocktails were obtained from Roche (Basel, Switzerland). Acrylamide was obtained from BioRad and the polyvinylidene difluoride membranes were from Millipore Corporation (Bedford, MA). The alkaline phosphatase-linked secondary antibodies and the enhanced chemifluorescence reagent were obtained from GE Healthcare (Chalfont St. Giles, UK). The antibody iNOS was from R\&D Systems (Abingdon, UK), COX-2 antibody was from Abcam (Cambridge, UK) and anti- $\beta$-tubulin was from Sigma Chemical Co. (St. Louis, MO, USA). The sodium hypochlorite ( $\mathrm{NaOCl}$ ) and boric acid were obtained from MaiaLab (Gondomar, Portugal). The naphthylethyldiamine, phosphoric acid $\left(\mathrm{H}_{3} \mathrm{PO}_{4}\right)$ and sulfuric acid $\left(\mathrm{H}_{2} \mathrm{SO}_{4}\right)$ were obtained from Panreac Quimica S.A.U (Barcelona, Spain). The sulphanilamide was obtained from Merck (Darmstadt, Germany). The enzyme soybean lipoxygenase (5-LOX), sodium nitroprusside (SNP), sodium linoleate, 5,5'-dithiobis-(2-nitrobenzoic acid) (DTNB), ethylenediamine tetraacetic acid (EDTA), sodium borohydrate $\left(\mathrm{NaBH}_{4}\right)$ and ascorbic acid were obtained from Sigma Chemical Co. (Saint Louis, USA).

\section{Plant extract}

The purified ethanolic extract of $C$. multiflorus was prepared using a hidroethanolic solution, and further purified onto C18 cartridges ( $2 \mathrm{~g}$, Waters, Milford, MA, USA), following the general procedures described in sections 3.2 and 3.3 [8]. The obtained purified extract $(\mathrm{CME})$ contained $410 \pm 8 \mathrm{mg}$ of polyphenols $/ \mathrm{g}$ of the extract, which included major amounts of chrysin-7-O-ß-D-glucopyranoside and of a dihydroxyflavone isomer of chrysin. It also contained considerable amounts of hexoside derivatives of the flavones luteolin, apigenin and of the flavonol quercetin [5, 8].

\section{HOCl scavenging assay}

This assay is based on the ability of hypochlorous acid ( $\mathrm{HOCl})$ to promote oxidation of thionitrobenzoic acid (TNB) to dithionitrobenzoic acid (DTNB). The TNB oxidation (followed by the absorbance decrease at $412 \mathrm{~nm}$ ) can be prevented when a $\mathrm{HOCl}$ scavenger compound is present in the reaction mixture. $\mathrm{HOCl}$ was prepared 
immediately before use by diluting the $\mathrm{NaOCl}$ solution to $0.1 \%(v / v)$ and adjusting its $\mathrm{pH}$ to 6.2 , with diluted sulfuric acid solution $\left(\mathrm{H}_{2} \mathrm{SO}_{4} 0.5 \mathrm{M}\right)$. The concentration of $\mathrm{HOCl}$ was further determined spectrophotometrically at $235 \mathrm{~nm}$ using the equation $[\mathrm{HOCl}]_{235}=$ A $/ 100 \mathrm{M}^{-1} \mathrm{~cm}^{-1}$. TNB was prepared by incubating $1 \mathrm{mM}$ DTNB in $50 \mathrm{mM}$ potassium phosphate buffer pH 6.6 (supplemented with $5 \mathrm{mM}$ EDTA) with $20 \mathrm{mM} \mathrm{NaBH}_{4}$ for 30 $\min$ at $37^{\circ} \mathrm{C}$. The concentration of TNB was determined by measuring the absorbance at $412 \mathrm{~nm}$ and using the equation $[\mathrm{TNB}]_{412}=\mathrm{A} / 13,600 \mathrm{M}^{-1} \mathrm{~cm}^{-1}$. The assays were performed at room temperature and the reaction mixtures ( $1 \mathrm{~mL}$ final volume) contained plant extract at various concentrations (10 to $200 \mu \mathrm{g} / \mathrm{mL})$, TNB $(70 \mu \mathrm{M})$ and $\mathrm{HOCl}(125 \mu \mathrm{M})$. The absorbance was measured at $412 \mathrm{~nm}$ using a Xion 500 photometer (Dr Lange, Germany), 5 min after the addition of $\mathrm{HOCl}$.

\section{Nitric oxide $\left(\mathrm{NO}^{\circ}\right)$ scavenging activity}

The NO ${ }^{\bullet}$ scavenging activity was assessed using the method described by Sousa et al. [9], with slight modifications. Briefly, $200 \mu \mathrm{L}$ of the six concentrations of CME (10 to 330 $\mu \mathrm{g} / \mathrm{mL}$ ) were added to $200 \mu \mathrm{L}$ of sodium nitroprusside (SNP, $10 \mathrm{mM}$ ). After $60 \mathrm{~min}$ of incubation, $200 \mu \mathrm{L}$ of Griess reagent $(1 \%$ sulphanilamide and $0.1 \%$ naphthylethyldiamine in $\left.2 \% \mathrm{H}_{3} \mathrm{PO} 4\right)$ was added to each tube and the mixture was incubated at room temperature for $10 \mathrm{~min}$, under light. The absorbance of the chromophore was measured at $562 \mathrm{~nm}$ using a Xion 500 photometer (Dr Lange, Germany). The $\mathrm{NO}^{\bullet}$ scavenging effect was expressed as $\mathrm{EC}_{50}$, indicating the extract concentration providing $50 \%$ inhibition.

\section{Culture of RAW 264.7 cells}

The mouse monocytic macrophage cell line RAW 264.7 was obtained from American Type Culture Collection (ATCC, USA) and supplied by Dr. Otília Vieira (Centro de Neurociências e Biologia Celular, Universidade de Coimbra, Coimbra, Portugal). The RAW 264.7 cells were maintained in Dulbecco's Modified Eagle Medium supplemented with $3.5 \mathrm{~g} / \mathrm{L}$ of glucose $(4.5 \mathrm{~g} / \mathrm{L}$ final concentration) with $10 \%$ non-inactivated fetal bovine serum, $100 \mathrm{U} / \mathrm{mL}$ penicillin, and $100 \mu \mathrm{g} / \mathrm{mL}$ streptomycin, at $37{ }^{\circ} \mathrm{C}$ in a humidified atmosphere of $95 \%$ air and $5 \% \mathrm{CO}_{2}$. 


\section{Determination of cell viability by MTT assay}

The potential cytotoxicity of the extract CME was measured by the 3-(4,5dimethylthiazol-2-yl)-2,5-diphenyl tetrazolium bromide (MTT) reduction colorimetric assay, as previously reported [10]. Cells $\left(0.3 \times 10^{6}\right.$ cells/well) were cultured in 48-well tissue culture plates and incubated for $12 \mathrm{~h}$ at $37^{\circ} \mathrm{C}$ with $5 \% \mathrm{CO}_{2}$. Thereafter, these were incubated in the absence or in the presence of CME (160 and $325 \mu \mathrm{g} / \mathrm{mL}$ ) for $1 \mathrm{~h}$ and further stimulated with $1 \mu \mathrm{g} / \mathrm{mL}$ LPS for $24 \mathrm{~h}$. Upon that, $43 \mu \mathrm{L}$ of MTT solution (5 $\mathrm{mg} / \mathrm{mL}$ in PBS) was added to each well and incubated for 15 min at $37^{\circ} \mathrm{C}$, in a humidified atmosphere of $95 \%$ air and $5 \% \mathrm{CO}_{2}$. The formazan produced by the metabolic activity of the cells was then dissolved in acidic isopropanol $(0.04 \mathrm{~N} \mathrm{HCl}$ in isopropanol) after supernatant removal. The absorbance was measured at $570 \mathrm{~nm}$, using an ELISA automatic microplate reader (SLT, Austria), with a reference wavelength of $620 \mathrm{~nm}$.

\section{Measurement of nitrite production in culture Raw 264.7 cells}

The amount of nitrite (an oxidative product of nitric oxide, $\mathrm{NO}^{\circ}$ ) in the culture supernatants, was determine by Griess reagent [11] as previous reported [12]. Briefly, $170 \mu \mathrm{l}$ of culture supernatants were collected and diluted with equal volumes of the Griess reagent $[0.1 \%(\mathrm{w} / \mathrm{v}) \mathrm{N}$-(1-naphthyl)-ethylenediamine dihydrochloride and $1 \%$ (w/v) sulphanilamide containing $5 \%(\mathrm{w} / \mathrm{v}) \mathrm{H}_{3} \mathrm{PO}_{4}$ ] and maintained during $30 \mathrm{~min}$ in the dark. The absorbance at $550 \mathrm{~nm}$ was measured in an automated plate reader (SLT, Austria) and the nitrite concentration determined from a sodium nitrite standard curve.

\section{Determination of the levels of iNOS and COX-2 by Western Blot analysis}

Raw 264.7 cells $\left(24 \times 10^{5}\right.$ cells/well) were sowed in 12-well plates to prepare total cell lysates for Western Blot analysis and allowed to stabilize. After $12 \mathrm{~h}$, cells were either maintained in culture medium (control) or pre-incubated with CEM for $1 \mathrm{~h}$. LPS (1 $\mu \mathrm{g} / \mathrm{mL}$ ) was thereafter added to the incubation medium and the cells were incubated for further $24 \mathrm{~h}$. After this period, cells were lysed with RIPA buffer $(50 \mathrm{mM}$ Tris $-\mathrm{HCl}, \mathrm{pH}$ 8.0, $1 \%$ Nonidet P-40, $150 \mathrm{mM} \mathrm{NaCl}, 0.5 \%$ sodium deoxycholate, $0.1 \%$ sodium dodecyl sulfate and $2 \mathrm{mM}$ ethylenediamine tetraacetic acid) freshly supplemented with $1 \mathrm{mM}$ dithiothreitol, protease and phosphatase inhibitor cocktails and sonicated in Vibra Cell sonicator (Sonics \& Material INC.) 
The nuclei and the insoluble cell debris were removed by centrifugation at $4{ }^{\circ} \mathrm{C}$, at $12,000 \times \mathrm{g}$ for $10 \mathrm{~min}$. The postnuclear extracts were collected and used as total cell lysates. Protein concentration was determined by the bicinchoninic acid protein assay [13] and cell lysates were denaturated in sample buffer $(0.125 \mathrm{mM}$ Tris $\mathrm{pH} 6.8,2 \%$ $(w / v)$ sodium dodecyl sulfate, $100 \mathrm{mM}$ dithiothreitol, $10 \%$ glycerol and bromophenol blue).

The levels of iNOS and COX-2 of the prepared cell lysates were performed by Western blot analysis, following the general procedure previous reported by Figueirinha et al. [14]. For that, an equivalent amount of protein were separated by $10 \%(\mathrm{v} / \mathrm{v})$ SDSPAGE followed by Western blotting. To examine the different proteins studied, the blots were incubated overnight at $4 \stackrel{\circ}{\circ}$ with the respective primary antibodies: COX-2 $(1: 10,000)$ and iNOS (1:5000). Protein detection was performed using the enhanced chemifluorescence system in the imager Thyphoon ${ }^{\mathrm{TM}}$ FLA 9000 (GE Healthcare). The bands densitometry were analyzed using the software ImageQuant TL®. $\beta$-tubulin was used as the reference protein.

\section{5-LOX inhibition assay}

5-Lipoxygenase (EC.1.13.11.12) is known to catalyze the oxidation of unsaturated fatty acids containing 1-4 diene structures. The conversion of linolenic acid to 1-3-linolenic acid hydroperoxide is followed spectrophotometrically by the appearance of a conjugated diene at $234 \mathrm{~nm}$. The reaction mixture contained $1.95 \mathrm{~mL}$ of sodium linoleate $(250 \mu \mathrm{M})$ and $10 \mu \mathrm{l}$ of CME or of the reference compound (ascorbic acid). The reaction was initiated by the addition of $50 \mu \mathrm{L}$ of soybean lipoxygenase solution ( 400 units $/ \mathrm{mL}$ in potassium borate buffer $0.2 \mathrm{M} \mathrm{pH} 8$ ). Changes in absorbance at $234 \mathrm{~nm}$ were measured for $5 \mathrm{~min}$. The percentage inhibition of the enzyme activity was calculated by comparison with the control.

\section{Statistical analysis}

All the experiments were performed in triplicate. Results are presented as mean $\pm S E M$ of the indicated number of experiments. To compare the effect of different treatments to LPS-stimulated cells, a multiple group comparison was performed and one-way ANOVA followed by Dunnett's test was used. The statistical tests were applied using GraphPad Prism, version 5.04 (GraphPad Software, San Diego, CA, USA). The significance level was $\# p<0.05$, \#\#p $<0.01$ and \#\#\#p $<0.001$, when compared to control and ${ }^{*} p<0.05,{ }^{* *} p<0.01$ and ${ }^{* *} p<0.001$, when compared to LPS. 


\subsubsection{Results and Discussion}

Despite the various biological activities that have been claimed for $C$. multiflorus plant $[2,3]$, up to present, scientific data has only been gathered for the antioxidant capacity of ethanolic extracts from different parts of the plant. 2,2-diphenyl-2-picrylhydrazyl $\left(\mathrm{DPPH}^{\bullet}\right)$ radical scavenging and the reducing iron (III) capacities were monitored together with the reactive oxygen species (ROS) scavenging capacity in HepG2 cells $[5,15]$. In turn, we herein intend to clarify the anti-inflammatory activities of $C$. multiflorus, and their underlying mechanisms of action.

\section{$\mathrm{HOCl}$ and $\mathrm{NO}^{\circ}$ scavenging activities}

Several inflammatory conditions have been related to the overproduction of oxygen (superoxide anion, perhydroxyl radical, protonated superoxide) and nitrogen (nitric oxide, nitrogen dioxide) free radicals. Also, other non radicals, namely hydrogen peroxide, singlet oxygen and hypochlorous acid or peroxynitrite anion and peroxynitrous acid have been included in ROS and RNS species, respectively $[6,16]$. $\mathrm{HOCl}$ is a strong oxidant produced in large amounts by neutrophils and its inhibition is frequently used as marker of anti-inflammatory potential. In a similar way, $\mathrm{NO}^{\bullet}$ scavenging ability has been largely assessed for plants due to its important role as a reactive specie mediator. Indeed, despite the NO ${ }^{\bullet}$ protective effects in physiological conditions, its uncontrolled production is associated to the amplification of inflammation and to tissue damage in inflammatory processes [17].

According to the experimental data (Table 1), the CME showed no relevant scavenging ability for $\mathrm{HOCl}$ species, with an $\mathrm{EC}_{50}$ value of about fifteen times higher than that of quercetin. Still, the extract provided significant protection against $\mathrm{NO}^{\bullet}$ production with $E C_{50}$ of $148.0 \pm 5.2 \mu \mathrm{g} / \mathrm{mL}$, which is less than the half of the $E_{50}$ of the ascorbic acid, used as reference compound 
Table 1 - Scavenging abilities of $\mathrm{HOCL}$ and $\mathrm{NO}^{\bullet}$ of $\mathrm{CME}$.

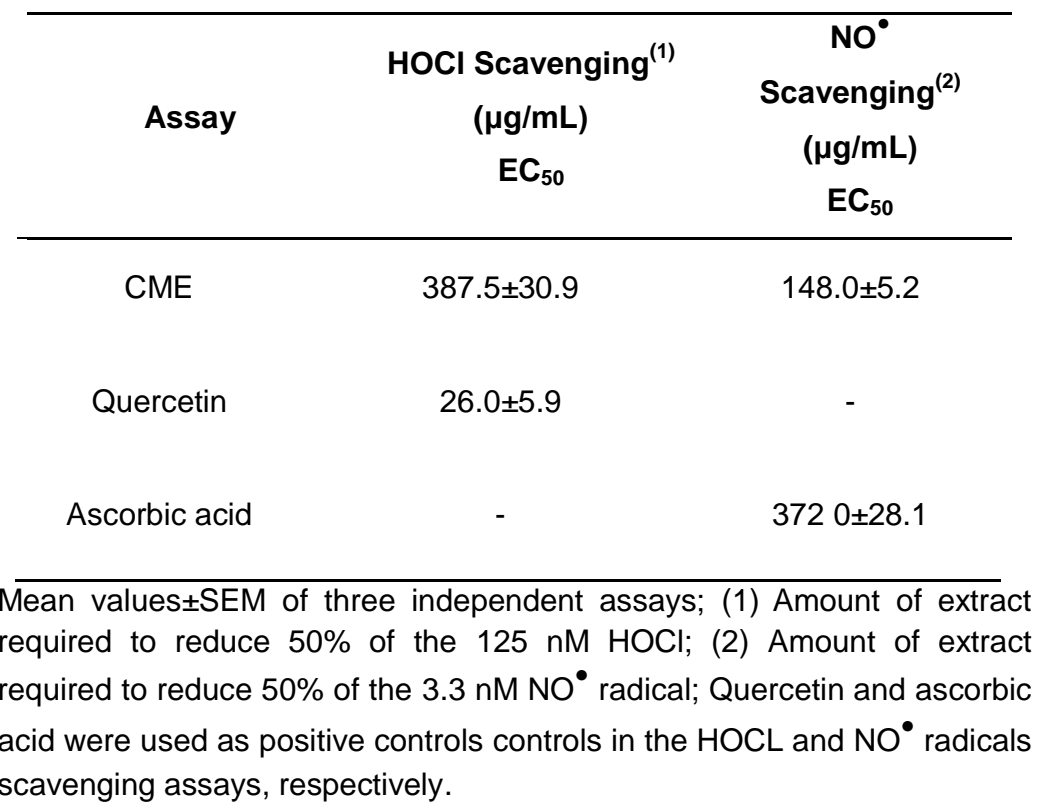

To our knowledge, the $\mathrm{NO}^{\bullet}$ scavenging ability of Cytisus species has only been described for $C$. scoparius hydroalcoholic extract $\left(E C_{50}=116 \mu \mathrm{g} / \mathrm{mL}\right)$ which is close to that herein obtained [18].

Furthermore, we also addressed the anti-inflammatory properties of the extract, using an in vitro model of inflammation, the mouse macrophage cell line, Raw 264.7, able to produce NO after lipopolysaccharide (LPS) triggering. NO is synthesized from Iarginine by inducible nitric oxide synthase (iNOS) expression in numerous mammalian cells, such as macrophages, and large amounts of NO have been found in several inflammatory-related diseases. For this reason, $\mathrm{NO}$ is a well established marker of inflammation, and inhibition of its production upon activation with an inflammatory stimulus, such as LPS, might be a useful strategy to disclose new anti-inflammatory drugs. In culture, the $\mathrm{NO}^{\bullet}$ released by the macrophages into the medium is converted to several nitrogen derivatives, from which only nitrite is stable, being easily measured by the Griess reagent [19].

In the cellular model, the $\mathrm{NO}^{\bullet}$ scavenging ability and the remaining effects were monitored for two non toxic concentrations (as evaluated by MTT assay, see Table 2) of CME, namely 160 and $325 \mu \mathrm{g} / \mathrm{mL}$. 
Table 2 - Effect of CME in Raw 264.7 macrophages viability.

\begin{tabular}{lc}
\hline Condition & $\begin{array}{c}\text { Cell Viability } \\
\text { (\% of control) }\end{array}$ \\
\hline Control & 100 \\
LPS $1 \mu \mathrm{g} / \mathrm{mL}$ & $82.3 \pm 1.1$ \\
$\mathrm{CME} 325 \mu \mathrm{g} / \mathrm{mL}$ & $90.7 \pm 14.0$ \\
$\mathrm{CME} 325 \mu \mathrm{g} / \mathrm{mL}+\mathrm{LPS} 1 \mu \mathrm{g} / \mathrm{mL}$ & $86.9 \pm 4.1$ \\
$\mathrm{CME} 160 \mu \mathrm{g} / \mathrm{mL}$ & $104.7 \pm 4.1$ \\
$\mathrm{CME} 160 \mu \mathrm{g} / \mathrm{mL}+\mathrm{LPS} 1 \mu \mathrm{g} / \mathrm{mL}$ & $94.0 \pm 13.0$ \\
\hline Viability of Raw $264.7 \mathrm{macrophages}$ cells incubated without or with LPS 1 \\
$\mu \mathrm{g} / \mathrm{mL}$ and co-incubated with CME (160 or $325 \mu \mathrm{g} / \mathrm{mL})$. The results are \\
expressed as percentage of cell viability versus control and the Values are \\
means $\pm S E M$ of percentage from at least in 3 independent experiments \\
performed duplicate. CME, Cytisus multiflorus purified ethanolic extract; \\
LPS, lipopolysaccharide
\end{tabular}

As can be observed in Figure 1, the cells treatment with LPS resulted in a huge increase in nitrite production ( $>400 \%$ than control). This production was counteracted by the pretreatment of macrophages with $160 \mu \mathrm{g} / \mathrm{mL}$ and $325 \mu \mathrm{g} / \mathrm{mL}$ of CME (about $21 \%$ and $33 \%$, respectively). To the author's knowledge, there are no previous results describing this effect for Cytisus species.

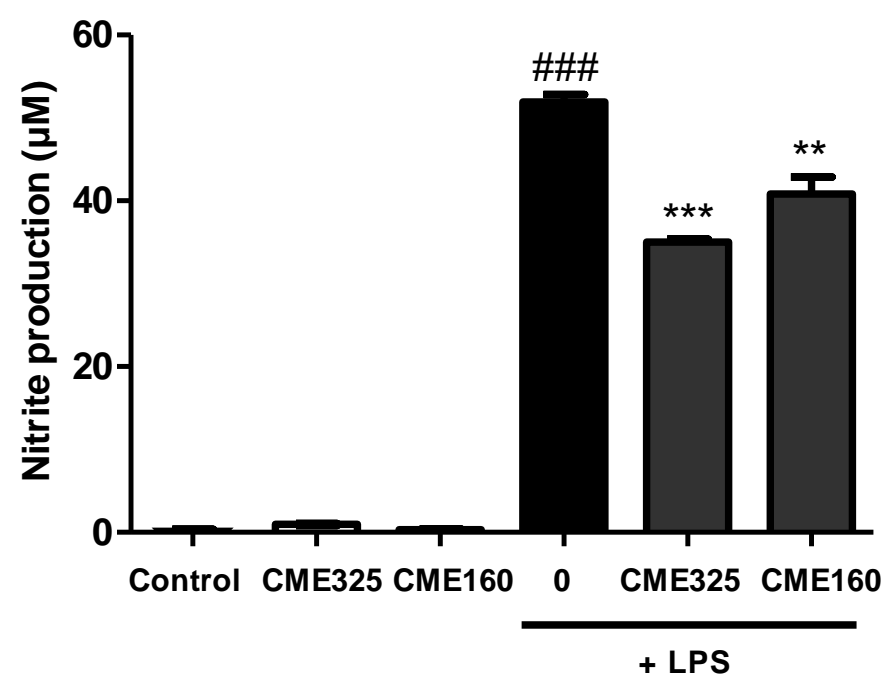

Figure 1 - Effect of $C$. multiflorus extract (CME) on NO production in RAW 264.7 macrophages. 
The macrophages were maintained in culture medium (control) or incubated with $1 \mu \mathrm{g} / \mathrm{mL}$ LPS, in the presence of CME 160 and $325 \mu \mathrm{g} / \mathrm{mL}$ for $24 \mathrm{~h}$. Nitrite levels in the culture supernatants were evaluated by the Griess reaction. Results are expressed as a percentage of nitrite production by control cells maintained in culture medium. Each value represents the mean \pm SEM from 3 experiments, performed in duplicate. Statistical analysis was performed by one-way ANOVA, followed by Dunnett's test. ${ }^{* *} p<0.01$, ${ }^{* * *} p<0.001$ when compared to cells exposed to LPS, in the absence of extract; \#\#\#p $<0.001$ when compared to untreated cells (control).

\section{Effect of CME on LPS-induced COX-2 and iNOS protein expression}

Once iNOS and COX-2 stimulate the production of large amounts of pro-inflammatory mediators (e.g. nitric oxide, prostaglandins), their inhibition are potential targets to prevent or treat chronic inflammation. In this sense, the expression of iNOS and COX-2 on LPS-induced mouse macrophages was analyzed by Western blot, using specific antibodies against iNOS and COX-2.

As depicted in Figure 2A, the iNOS protein expression was much raised when the cells were incubated for $24 \mathrm{~h}$ with LPS, comparing to non-stimulated RAW 264.7 cells (control). A significant reduction in the iNOS expression (26\%) was observed, after the cells treatment with CME $325 \mu \mathrm{g} / \mathrm{mL}$.

The exposure of RAW 264.7 cells to LPS induced a significant increase in COX-2 levels and this was not significantly changed by the presence of CME extract, for both concentrations (Figure 2B). The inhibition of iNOS expression (and the ineffectiveness in protecting from COX-2 increased expression) has been described for other plant extracts or for pure phenolic compounds [20]. To the author's knowledge, this is the first work demonstrating iNOS expression inhibition of Cytisus plants.

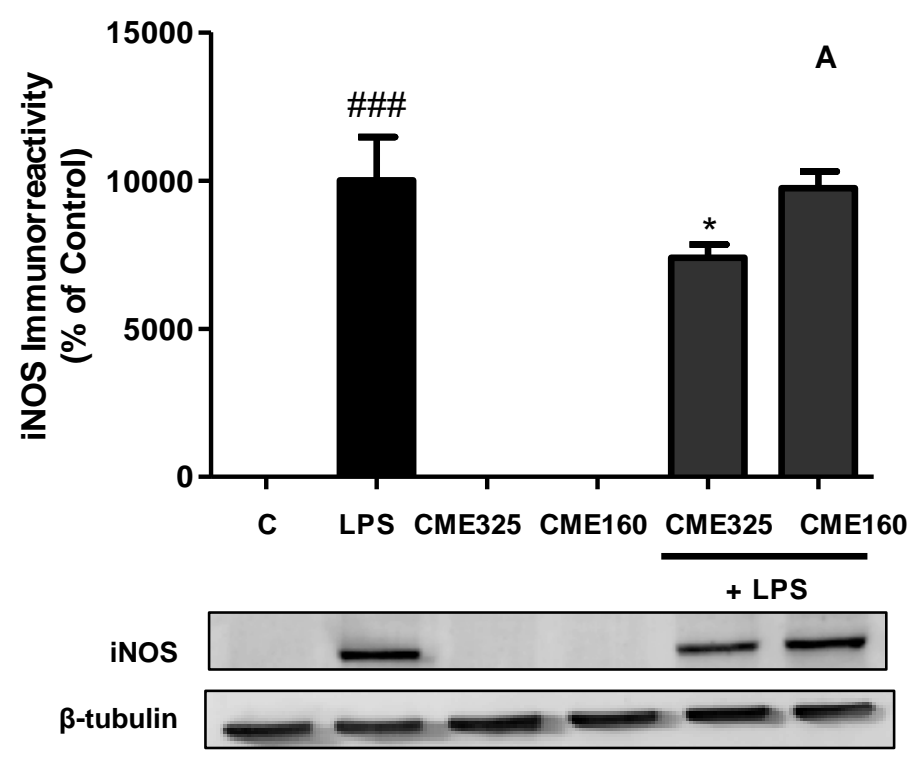




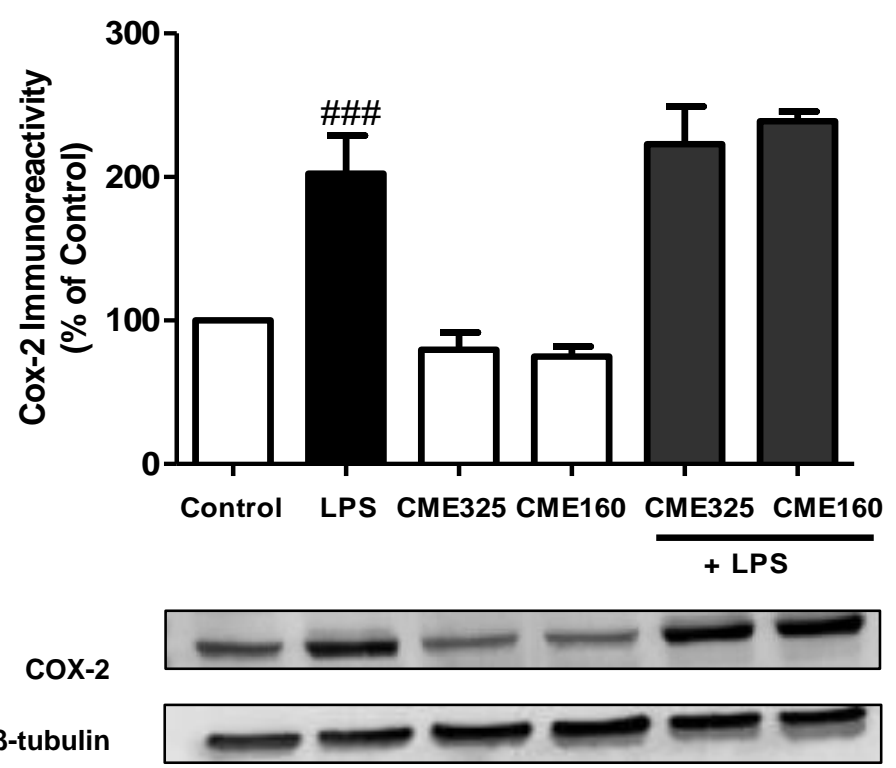

Figure 2 - Effect of $C$. multiflorus extract (CME) 325 and $160 \mu \mathrm{g} / \mathrm{mL}$ in the iNOS (A) or COX-2 protein levels (B) in macrophages stimulated with LPS $1 \mu \mathrm{g} / \mathrm{mL}$.

Raw 264.7 cells were maintained in culture medium (control), or pre-incubated for $1 \mathrm{~h}$ with CME 325 and $160 \mu \mathrm{g} / \mathrm{mL}$, and then treated with $1 \mu \mathrm{g} / \mathrm{mL}$ LPS for $24 \mathrm{~h}$. Total cell extracts were analyzed by Western blot using a specific anti- iNOS antibody (A) and anti-COX-2 antibody (B). Anti- $\beta$-tubulin antibody was used to protein normalization. The blot shown is representative of 4 blots yielding similar results. Results were expressed as percentage of COX-2 (A) or iNOS (B) protein levels relatively to control. Each value represents the mean $\pm S E M$ from 3 independent experiments. Statistical analysis was performed by oneway ANOVA, followed by Dunnett's test. ${ }^{*} p<0.05$ when compared to cells exposed to LPS, in the absence of extract; \#\#\#p 0.001 when compared to untreated cells (control).

\section{Effect of CME on 5-LOX inhibition}

Lipoxygenases (LOX) are enzymes responsible for generating leukotrienes $\left(\mathrm{LTC}_{4}\right.$, $\mathrm{LTD}_{4}, \mathrm{LTE}_{4}$ ) which are, with prostaglandins, the strong mediators of inflammation. In the present study, the inhibition of 5-LOX enzyme activity by the CME $(7.5$ to $60 \mu \mathrm{g} / \mathrm{mL})$ was recorded together with that of ascorbic acid $(2.5$ to $10 \mu \mathrm{g} / \mathrm{mL})$, that is a potent inhibitor of the enzyme and for that was used as control [21]. As shown in Fig. 3, the CME partially inhibited the activity of 5-LOX, with a maximum of inhibition of about $30 \%$ and an $\mathrm{EC}_{25}$ value of $37.90 \mu \mathrm{g} / \mathrm{mL}$. 


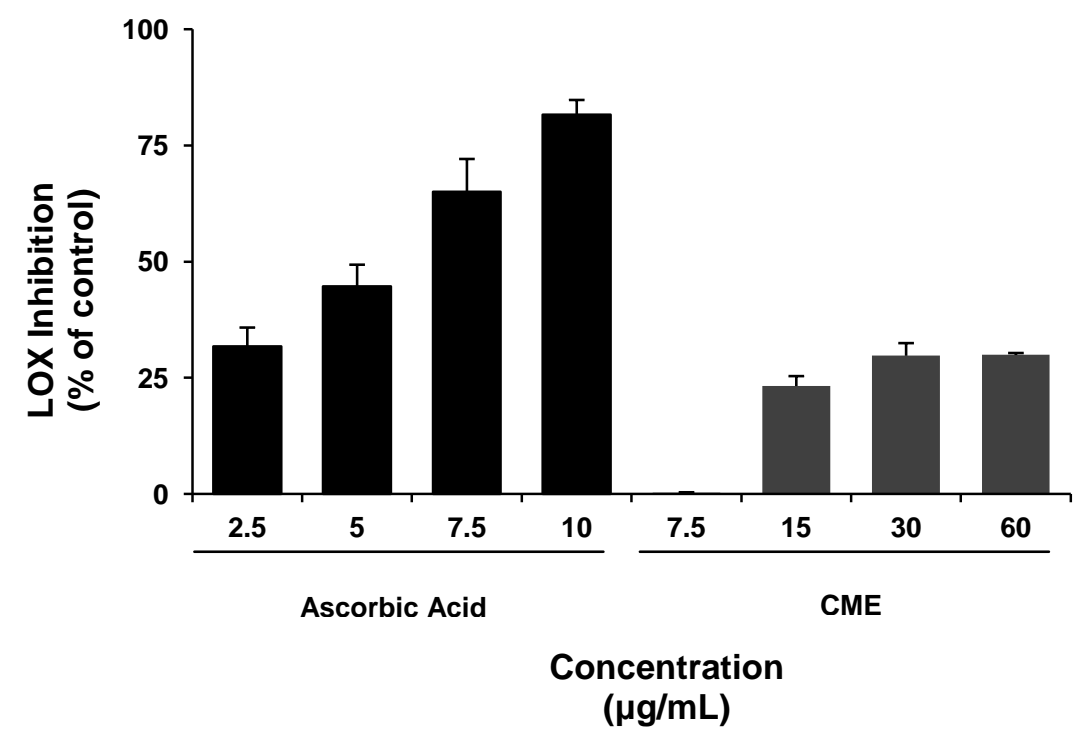

Figure 3 - Effect of ascorbic acid and of $C$. multiflorus extract (CME) on the activity of 5-LOX

Radical scavenging and inhibition of ROS production are common mechanism of action in polyphenols for preventing the propagation of the oxidizing chain reaction and, consequently, the damage of macromolecules. These actions are frequently related to their anti-inflammatory activities [22]. Other anti-inflammatory related mechanisms of polyphenols include the decrement of inflammatory cells, namely macrophages. Macrophages produce soluble mediators, namely cytokines, and chemokines, which act by further recruiting inflammatory cells to the site of damage and producing more reactive species. These key mediators can activate signal transduction cascades as well as inducing changes in transcription factors, which mediate immediate cellular inflammatory and stress responses. The main pathway initiating this inflammatory process is the nuclear factor NF-kB signaling pathway. Activation of the transcriptional factor NF-kB causes induction of COX-2 and iNOS and aberrant expression of inflammatory cytokines, which have been reported to play a role in oxidative stressinduced inflammation. Several authors reported the effect of polyphenols in the NF-kB signaling pathways with modulation of proinflammatory enzymes and with consequent decrement of inflammatory mediators such as $\mathrm{NO}^{\circ}$, leukotrienes, TNF- $\alpha$ and interleukins [22, 23].

As previously described, CME extract is rich in flavonoids and particularly abundant in chrysin and derivatives. Literature data indicates that chrysin suppresses the proinflammatory enzymes COX-2 and iNOS activities and can counteract the increased levels of the proinflammatory cytokines IL-6, TNF- $\alpha$, as well as PGE 2 [24-27]. CME also 
contains moderate amounts of derivatives of the luteolin, apigenin and quercetin for which anti-inflammatory effects have been demonstrated [20]. Concretely, luteolin and quercetin inhibited NO*, TNF- $\alpha$ and IL- 6 release while the first has shown inhibitory activity against COX-2. Apigenin inhibits the production of NO', IL-1 $\beta, I L-8$, TNF- $\alpha$ and $\mathrm{PGE}_{2}$ by suppressing the expression of iNOS and COX-2 [20, 22, 27, 28].

Overall, the present results showed that the CME has the ability to partially scavenge the $\mathrm{NO}^{\bullet}$ radical scavenging (in chemical and in a cell model), as well as to reduce the expression of iNOS and 5-LOX. Since accumulation of reactive oxygen and nitrogen species generated by inflammatory cells that created oxidative stress is thought to be one of the major factor by which chronic inflammation contributes to chronic diseases, these results suggest that $C$. multiflorus exert an anti-inflammatory action with potential application in inflammatory related diseases. This hypothesis needs to be supported by in vivo models.

\subsubsection{References}

[1] Basu, S.; Hazra, B., Evaluation of nitric oxide scavenging activity, in vitro and ex vivo, of selected medicinal plants traditionally used in inflammatory diseases. Phytotherapy Research, 2006, 20(10), 896-900.

[2] Gião, M. S.; Gonzalez-Sanjose, M. L.; Rivero-Perez, M. D.; Pereira, C. I.; Pintado, M. E.; Malcata, F. X., Infusions of Portuguese medicinal plants: Dependence of final antioxidant capacity and phenol content on extraction features. Journal of the Science of Food and Agriculture, 2007, 87(14), 2638-2647.

[3] Carvalho, A. M., Plantas y sabiduría popular del Parque Natural de Montesinho. Un estudio etnobotánico en Portugal. Consejo Superior de Investigaciones Científicas. Biblioteca de Ciencias no 35: Madrid, 2010.

[4] Prieto, J. M.; Bader, A.; Martini, F.; Rios, J. L.; Morelli, I., Screening of some rare endemic Italian plants for inhibitory activity on 5-lipoxygenase. Fitoterapia, 2005, 76(78), 725-727.

[5] Pereira, O. R.; Macias, R. I. R.; Perez, M. J.; Marin, J. J. G.; Cardoso, S. M., Protective effects of phenolic constituents from Cytisus multiflorus, Lamium album L. and Thymus citriodorus on liver cells. Journal of Functional Foods, 2013, in press.

[6] Cuzzocrea, S.; Riley, D. P.; Caputi, A. P.; Salvemini, D., Antioxidant therapy: A new pharmacological approach in shock, inflammation, and ischemia/reperfusion injury. Pharmacological Reviews, 2001, 53(1), 135-159.

[7] Kris-Etherton, P. M.; Lefevre, M.; Beecher, G. R.; Gross, M. D.; Keen, C. L.; Etherton, T. D., Bioactive compounds in nutrition and health-research methodologies for establishing biological function: The antioxidant and anti-inflammatory effects of flavonoids on atherosclerosis. Annual Review of Nutrition, 2004, 24, 511-538. 
[8] Pereira, O. R.; Silva, A. M. S.; Domingues, M. R. M.; Cardoso, S. M., Identification of phenolic constituents of Cytisus multiflorus. Food Chem., 2012, 131(2), 652-659.

[9] Sousa, C.; Valentao, P.; Ferreres, F.; Seabra, R. M.; Andrade, P. B., Tronchuda Cabbage (Brassica oleracea L. var. costata DC): Scavenger of Reactive Nitrogen Species. Journal of Agricultural and Food Chemistry, 2008, 56(11), 4205-4211.

[10] Mosmann, T., Rapid colorimetric assay for cellular growth and survival: Application to proliferation and cytotoxicity assays. Journal of Immunological Methods, 1983, 65(12), 55-63.

[11] Green, L. C.; Wagner, D. A.; Glogowski, J.; Skipper, P. L.; Wishnok, J. S.; Tannenbaum, S. R., Analysis of nitrate, nitrite, and [N-15]-labeled nitrate in biologicalfluids. Analitical Biochemistry, 1982, 126(1), 131-138.

[12] Francisco, V.; Figueirinha, A.; Neves, B. M.; Garcia-Rodriguez, C.; Lopes, M. C.; Cruz, M. T.; Batista, M. T., Cymbopogon citratus as source of new and safe antiinflammatory drugs: Bio-guided assay using lipopolysaccharide-stimulated macrophages. Journal of Ethnopharmacology, 2011, 133(2), 818-827.

[13] Smith, P. K.; Krohn, R. I.; Hermanson, G. T.; Mallia, A. K.; Gartner, F. H.; Provenzano, M. D.; Fujimoto, E. K.; Goeke, N. M.; Olson, B. J.; Klenk, D. C., Measurement of protein using bicinchoninic acid. Anal. Biochem., 1985, 150(1), 76-85.

[14] Figueirinha, A.; Paranhos, A.; Perez-Alonso, J. J.; Santos-Buelga, C.; Batista, M. T., Cymbopogon citratus leaves: Characterisation of flavonoids by HPLC-PDA$\mathrm{ESI} / \mathrm{MS} / \mathrm{MS}$ and an approach to their potential as a source of bioactive polyphenols. Food Chemistry, 2008, 110(3), 718-728.

[15] Luis, A.; Domingues, F.; Duarte, A. P., Bioactive Compounds, RP-HPLC Analysis of Phenolics, and Antioxidant Activity of Some Portuguese Shrub Species Extracts. Natural Product Communications, 2011, 6(12), 1863-1872.

[16] Gomes, A.; Fernandes, E.; Lima, J., Fluorescence probes used for detection of reactive oxygen species. Journal of Biochemical and Biophysical Methods, 2005, 65(23), $45-80$.

[17] Hofseth, L. J., Nitric oxide as a target of complementary and alternative medicines to prevent and treat inflammation and cancer. Cancer Letters, 2008, 268(1), 10-30.

[18] Raja, S.; Nazeer, H.; Kumar, V.; Kakali, M.; Bishnu, S.; Arun, B.; Pulok, M., Cytisus scoparius link - A natural antioxidant. BMC Complementary and Alternative Medicine, 2006, 6(1), 1-7.

[19] Pinho, B. R.; Sousa, C.; Valentao, P.; Andrade, P. B., Is Nitric Oxide Decrease Observed with Naphthoquinones in LPS Stimulated RAW 264.7 Macrophages a Beneficial Property? Plos One, 2011, 6(8).

[20] Mueller, M.; Hobiger, S.; Jungbauer, A., Anti-inflammatory activity of extracts from fruits, herbs and spices. Food Chemistry, 2010, 122(4), 987-996.

[21] Hoobler, E. K.; Holz, C.; Holman, T. R., Pseudoperoxidase investigations of hydroperoxides and inhibitors with human lipoxygenases. Bioorganic \& Medicinal Chemistry, 2013, 21(13), 3894-3899. 
[22] Garcia-Lafuente, A.; Guillamon, E.; Villares, A.; Rostagno, M. A.; Martinez, J. A., Flavonoids as anti-inflammatory agents: implications in cancer and cardiovascular disease. Inflammation Reserach, 2009, 58(9), 537-552.

[23] Yoon, J. H.; Baek, S. J., Molecular targets of dietary polyphenols with antiinflammatory properties. Yonsei Medical Journal, 2005, 46(5), 585-596.

[24] Cho, H.; Yun, C.-W.; Park, W.-K.; Kong, J.-Y.; Kim, K. S.; Park, Y.; Lee, S.; Kim, B.-K., Modulation of the activity of pro-inflammatory enzymes, COX-2 and iNOS, by chrysin derivatives. Pharmacological Research, 2004, 49(1), 37-43.

[25] Rehman, M. U.; Tahir, M.; Khan, A. Q.; Khan, R.; Lateef, A.; Oday, O. H.; Qamar, W.; Ali, F.; Sultana, S., Chrysin suppresses renal carcinogenesis via amelioration of hyperproliferation, oxidative stress and inflammation: Plausible role of NF-ı̂o-B. Toxicology Letters, 2013, 216, 146-158.

[26] Anand, K. V.; Anandhi, R.; Pakkiyaraj, M.; Geraldine, P., Protective effect of chrysin on carbon tetrachloride (CCl4)-induced tissue injury in male Wistar rats. Toxicology and Industrial Health, 2011, 27(10), 923-933.

[27] Sergent, T. r. s.; Piront, N.; Meurice, J.; Toussaint, O.; Schneider, Y.-J., Antiinflammatory effects of dietary phenolic compounds in an in vitro model of inflamed human intestinal epithelium. Chemico-Biological Interactions, 2010, 188(3), 659-667.

[28] Gautam, R.; Jachak, S. M., Recent developments in anti-inflammatory natural products. Medicinal Research Reviews, 2009, 29(5), 767-820. 


\subsection{InfLUENCE Of MENTHA AQUATICA L. AND LEONURUS CARDIACA L. PURIFIED ETHANOLIC EXTRACTS IN MITOCHONDRIAL BIOENERGETICS}

Mitochondria play an important role in cell homeostasis, participating in the synthesis of ATP formed through oxidative phosphorylation, and in the biosynthesis of fatty acids or amino acids. Besides the metabolic functions, it is involved in calcium fluxes, ROS and RNS production and in cell signaling [1, 2]. Moreover, mitochondria are a recognize model for evaluating the action of xenobiotics on cell and have been used as biosensor to predict drug safety [3, 4].

Disturbances in mitochondrial bioenergetics are related to several mechanisms that lead to cell injury and are also associated with different dysfunctions, including neurodegenerative disorders and the so-called "mitochondrial diseases" [5]. In fact, apoptotic cell death is associated with mitochondrial DNA mutations, decreased production of ATP, formation of free radicals and alterations in cellular calcium fluxes that promote the peroxidation of mitochondrial macromolecules (DNA, proteins, and lipids) and the opening of the mitochondrial permeability transition pore. Due the important role in energy metabolism in cells, mitochondria are potential target of therapeutic substances. Actually, several drugs (e.g. antidiabetic, antiviral, antitumor, potassium channel openers and anesthetics) have their treating mechanism based on alterations of mitochondrial functions [2, 5]. In turn, drugs that have other cellular targets may also affect mitochondrial function, and this usually is associated with their side effects [5]. In this way, mitochondria bioenergetic measurements can be used to estimate the potential therapeutic applications of plant extracts, of isolated compounds or drugs, or alternatively, to estimate their cytotoxicity [5-8].

Mitochondrial bioenergetics monitoring can be evaluated in isolated mitochondria. Liver mitochondria are usually the starting point, as this organ is a key metabolic one in metabolism. Indeed, liver toxicity is a major worry in drug commercialization and several methods are established in order to assess possible toxic effects of compounds or plant extracts [3]. In recent decades, the increased demand of natural products as alternative or complementary therapeutic products is leading to an exponential search focusing medicinal plants and their bioactive compounds. Despite this, their effects on mitochondrial bioenergetics is until now overlooked. The potential therapeutic effects or, alternatively, the associated toxicity to their consumption is poorly studied. Hence, pharmacological studies are needed in order to determine the mechanisms associated with the beneficial activities and also the safe level of exposure of plants used as folk medicines. 


\section{4 | RESULTS AND Discussion}

Although Mentha aquatica L. and Leonurus cardiaca L. species have been used in pharmaceutical supplements and in several medical situations [9-11], the therapeutic effects are understudied and toxic activities have not been assessed [12].

In this way, the present work aimed to evaluate for the first time, the influence of $M$. aquatica and $L$. cardiaca purified ethanolic extracts in mitochondrial function. For that, mitochondrial bioenergetic assays were performed and the respiratory parameters state 2 , state 3 , state 4 , uncoupled respiration respiratory control ratio (RCR), and $P / O$ ratio, together with transmembrane potencial, were evaluated in the presence of two distinct concentrations of the plant extracts.

\subsubsection{Materials and Methods}

\section{Materials}

All chemicals used were of analytical grade and obtained from standard commercial sources. Inhibitors and drugs were dissolved in water or ethanol. In control experiments, solvents were added to isolated mitochondria at concentrations not exceeding $0.2 \%$.

\section{Plants extracts}

The purified ethanolic extracts were obtained from the aerial parts of Mentha aquatica L. and Leonurus cardiaca L., following the general procedure previous described [13]. As reported in sections 3.4 and 3.5, the $M$. aquatica and L. cardiaca purified ethanolic extracts (PEEMa and PEELc, respectively) have $302.5 \pm 8.0$ and $500.4 \pm 49.1 \mathrm{mg}$ of phenolic compounds/g of extract, respectively. The PEEMa is enriched in eriodictyol-7O-rutinoside (145 mg/g of extract) and rosmarinic (64 mg/g of extract) and also possesses moderate amounts of 7-O-rutinoside derivatives of luteolin, hesperidin and naringenin. In turn, the PEELC is enriched in phenylethanoid glycosides, which represents approximately $90 \%$ of the total phenolics quantified and mainly enclose lavandulifolioside and verbascoside (254 and $137 \mathrm{mg} / \mathrm{g}$ of extract, respectively). The extract also contain moderate amounts of glycosidic derivatives of quercetin (flavonol) representing $10 \%$ of the total quantified phenolics. 


\section{Isolation of rat liver mitochondria}

Wistar rats (200-250 g), with 3 months of age, were fasted overnight before being killed by cervical displacement. The isolation of mitochondria was performed by conventional methods [14], with minor modifications as previously described [15]. Homogenization medium was composed of $250 \mathrm{mM}$ sucrose, $10 \mathrm{mM} \mathrm{4-(2-Hydroxyethyl)piperazine-1-}$ ethanesulfonic acid (HEPES), pH 7.4, 1 mM ethylene-bis(oxyethylenenitrilo) tetraacetic acid (EGTA), and $0.1 \%$ fat-free bovine serum albumin (BSA). EGTA and bovine serum albumin (BSA) were omitted from the final washing medium, adjusted at $\mathrm{pH}$ 7.2. The mitochondrial pellet was washed twice, suspended in the washing medium, and immediately used [16]. The final concentration of mitochondrial protein was determined by the biuret method [17], using BSA as standard. The experiments were carried out in accordance with the National Requirements for Vertebrate Animal Research and the EU guidelines (2010/63/EU).

\section{Mitochondrial respiration}

Oxygen consumption of isolated mitochondria was measured polarographically at $30^{\circ} \mathrm{C}$ with a Clark oxygen electrode, in a closed chamber with magnetic stirring. The reaction medium consisted of $250 \mathrm{mM}$ sucrose, $20 \mathrm{mM} \mathrm{KCl}, 2 \mathrm{mM} \mathrm{MgCl}, 5 \mathrm{mM} \mathrm{KH}_{2} \mathrm{PO}_{4}$ and 5 $\mathrm{mM}$ Hepes ( $\mathrm{pH}$ 7.2). Mitochondria (1 mg protein), were added to $1 \mathrm{~mL}$ of the standard respiratory medium $\left(25^{\circ} \mathrm{C}\right)$ in the absence or in presence of PEEMa or PEELc and allowed to incubate for $5 \mathrm{~min}$ before the addition of $2 \mu \mathrm{M}$ rotenone. Respiration was started through the addition of $5 \mathrm{mM}$ glutamate/malate or $5 \mathrm{mM}$ succinate plus $3 \mu \mathrm{M}$ rotenone. State 3 was elicited by adding adenosine 5'-diphosphate (ADP $125 \mathrm{nmol}$ ) and state 4 respiration was achieved after full ADP phosphorylation [18]. For uncoupled respiration, $1 \mu \mathrm{M}$ FCCP (carbonyl cyanide $p$-trifluoromethoxyphenylhydrazone) was added after a phosphorylative cycle.

\section{Mitochondrial membrane potential}

The mitochondrial transmembrane potential $(\Delta \psi)$ was measured indirectly based on the lipophilic cation tetraphenylphosphonium $\left(\mathrm{TPP}^{+}\right)$activity, by using a $\mathrm{TPP}^{+}$-selective electrode in combination with and $\mathrm{Ag} / \mathrm{AgCl}$-saturated reference electrode, as previously described [19]. Mitochondria (1 $\mathrm{mg}$ protein) were incubated in $1 \mathrm{~mL}$ of medium containing $250 \mathrm{mM}$ sucrose, $20 \mathrm{mM} \mathrm{KCl}, 2 \mathrm{mM} \mathrm{MgCl}, 5 \mathrm{mM} \mathrm{KH}_{2} \mathrm{PO}_{4}$, and $5 \mathrm{mM}$ Hepes ( $\mathrm{pH}$ 7.2), supplemented with $3 \mu \mathrm{M} \mathrm{TPP}^{+}$in the absence or in presence of 
PEEMa or PEELc. After an incubation period of $5 \mathrm{~min}$, mitochondria were energized with glutamate + malate $(5 \mathrm{mM}+5 \mathrm{mM})$ or rotenone $(2 \mu \mathrm{M})$ and succinate $(5 \mathrm{mM})$. The baseline was achieved by adding valinomycin. No correction was made for the "passive" binding of $\mathrm{TPP}^{+}$to the mitochondria membranes because the purpose of the experiments was to show relative changes in potential rather than absolute values. As a consequence, we can anticipate some overestimation for the values.

\section{Statistics}

Solvent controls (water or ethanol) were included within each experimental determination, and the numerical data are expressed as a percentage of the respective control. Ethanol itself had no effect on any of the parameters measured. Statistical analyses were performed using GraphPad Prism 5 software (GraphPad, San Diego, $\mathrm{CA}$ ). The results were presented as mean \pm SEM of the number of experiments shown on tables and figures legends. Statistical significance was determined using one-way ANOVA, with Tukey post-test. $p<0.05$ was considered significant.

\subsubsection{Results and Discussion}

\section{Effects of PEEMa or PEELc on mitochondrial respiratory rates}

Fig. 1a shows a typical oxygen consumption record in control conditions, which reflect changes in the metabolic states of mitochondria. In state 1, mitochondria display a slow respiratory rate, presumably due to the metabolism of endogenous substrate and nucleotide. This rate is slightly increased in state 2 , due to the adding of exogenous substrate. In turn, the addition of ADP to state 2 activates oxidative phosphorylation which is characteristic of state 3 . Increased electron flux in the respiratory chain during state 3 metabolism is indicated by a temporary increase in the rate of oxygen consumption. State 4 is established by the deceleration of respiration that is observed upon the complete phosphorylation of available ADP. The low respiratory rate of state IV continues until all available oxygen in the reaction system is consumed. 


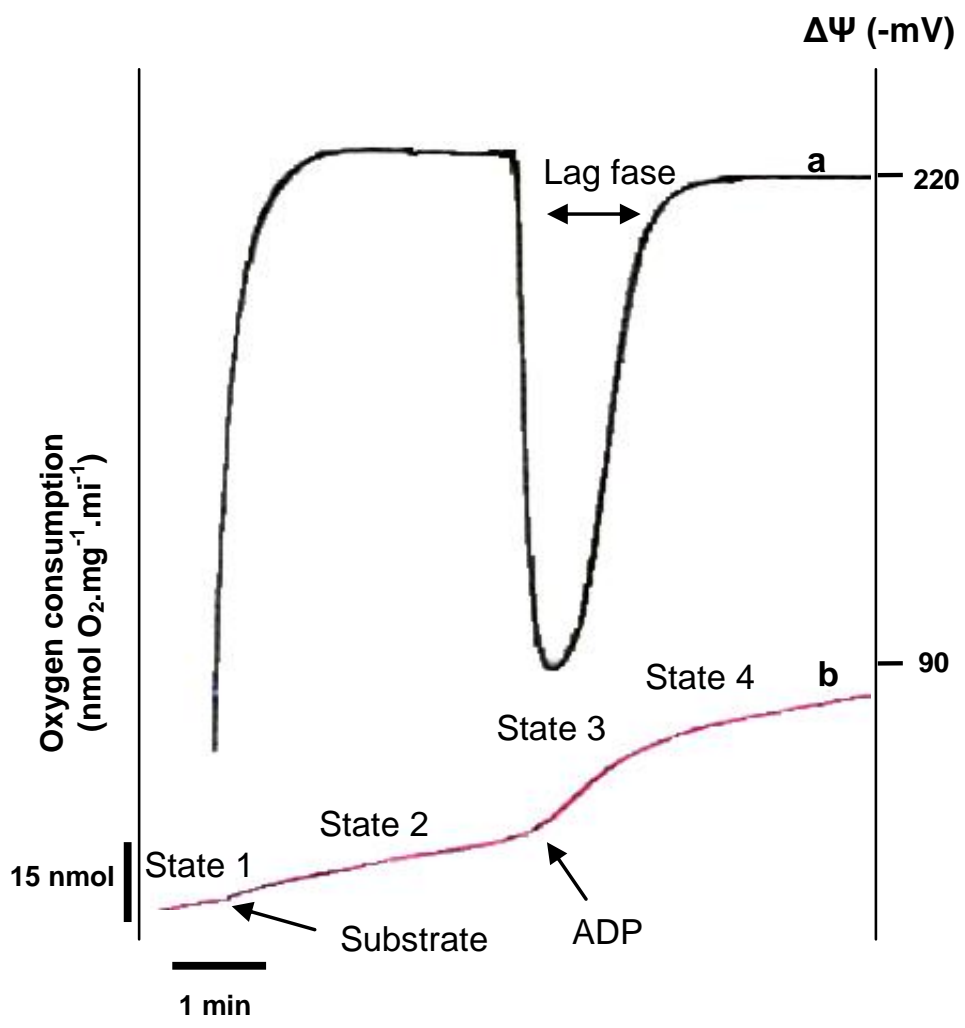

Figure 1 - Representative oxygen consumption trace (a) and mitochondrial electric potential (b) measured with a TPP ${ }^{+}$-selective electrode. Rat liver mitochondria $(1 \mathrm{mg})$ were incubated in 1 $\mathrm{mL}$ of the medium supplemented with $3 \mu \mathrm{M} \mathrm{TPP}{ }^{+}$. Energization of the mitochondrial population was achieved with $5 \mathrm{mM}$ succinate plus $2 \mu \mathrm{M}$ rotenone. After stabilization of the recording, 125 nmol ADP was added.

The effects of PEEMa and PEELc (15 and $25 \mu \mathrm{g} . \mathrm{mg}$ protein $^{-1}$ ) on the metabolic states of isolated mitochondria are resumed in Table 1. For the PEEMa, the results indicated a decrease in the respiratory state 3 , evaluated either in the presence of glutamate/malate or succinate. This effect was dose-dependent and statistically significant for $25 \mu \mathrm{g} . \mathrm{mg} \mathrm{protein}^{-1}$ and for both substrates. Respiratory state 4 was not significantly changed.

On the other hand, the mitochondria treatment with the same concentrations of the PEELc did not change any of the investigated respiratory parameters in the presence of complex I-linked substrates, although when complex II-linked substrate was used, a decreasing tendency on state 3 and an increasing tendency on state 4 were both observed, with significant differences being observed for the $25 \mu \mathrm{g} . \mathrm{mg}$ protein $^{-1}$. 
Moreover, under uncoupled respiration, which was induced by FCCP (a weak lipophilic acid that abolishes the proton electrochemical gradient, and maximally stimulates respiration) a decreased pattern similar to that of state 3 was registered, reflecting a decrease in the rate of electron transfer, more significant for higher concentrations.

Table 1 - Effects of the M. aquatica (PEEMa) and L. cardiaca (PEELc) purified ethanolic extracts on rat liver mitochondrial respiratory rates.

\begin{tabular}{|c|c|c|c|c|c|}
\hline $\begin{array}{l}\text { Plant } \\
\text { extract }\end{array}$ & Condition & $\begin{array}{c}\text { V2 } \\
\mathrm{nmol} \mathrm{O} \cdot \mathrm{mg}^{-} \\
{ }^{1} \cdot \mathrm{min}^{-1}\end{array}$ & $\begin{array}{c}\text { V3 } \\
\mathrm{nmol} \mathrm{O} \cdot \mathrm{mg}^{-} \\
{ }^{1} \cdot \mathrm{min}^{-1}\end{array}$ & $\begin{array}{c}\text { V4 } \\
\mathrm{nmol} \mathrm{O}_{2} \cdot \mathrm{mg}^{-} \\
{ }^{1} \cdot \mathrm{min}^{-1}\end{array}$ & $\begin{array}{c}\mathbf{V}_{\mathrm{FccP}} \\
\mathrm{nmol} \mathrm{O}_{2} \cdot \mathrm{mg}^{-} \\
{ }^{1} \cdot \mathrm{min}^{-1}\end{array}$ \\
\hline \multirow{8}{*}{ PEEMa } & \multicolumn{5}{|l|}{ Glutamate + Malate } \\
\hline & Control & $4.2 \pm 0.5$ & $40.5 \pm 1.9$ & $10.9 \pm 0.9$ & $42.6 \pm 1.2$ \\
\hline & $\begin{array}{l}\text { M. aquatica } \\
15 \mu \text { g.mg protein }{ }^{-1}\end{array}$ & $4.9 \pm 0.9$ & $35.0 \pm 1.5$ & $9.6 \pm 0.7$ & $32.0 \pm 1.6$ ** \\
\hline & $\begin{array}{l}\text { M. aquatica } \\
25 \mu \text { g.mg protein }{ }^{-1}\end{array}$ & $4.7 \pm 0.4$ & $30.7 \pm 1.8^{*}$ & $9.8 \pm 0.5$ & $24.9 \pm 1.9^{* *}$ \\
\hline & \multicolumn{5}{|l|}{ Succinate } \\
\hline & Control & $6.3 \pm 0.3$ & $26.1 \pm 1.0$ & $6.3 \pm 0.3$ & $32.6 \pm 1.9$ \\
\hline & $\begin{array}{l}\text { M. aquatica } \\
15 \mu \text { g.mg protein }{ }^{-1}\end{array}$ & $7.1 \pm 0.4$ & $23.6 \pm 1.1$ & $6.4 \pm 0.4$ & $25.4 \pm 3.4$ \\
\hline & $\begin{array}{l}\text { M. aquatica } \\
25 \mu \text { g.mg protein }{ }^{-1}\end{array}$ & $7.0 \pm 0.4$ & $21.8 \pm 1.25^{\star}$ & $6.5 \pm 0.4$ & $22.5 \pm 3.1^{*}$ \\
\hline \multicolumn{6}{|c|}{ Glutamate + Malate } \\
\hline \multirow{7}{*}{ PEELC } & Control & $4.1 \pm 2.0$ & $17.8 \pm 2.1$ & $4.4 \pm 0.4$ & nd \\
\hline & $\begin{array}{l}\text { L. cardiaca } \\
15 \mu \mathrm{g} . \mathrm{mg} \text { protein }^{-1}\end{array}$ & $4.8 \pm 0.6$ & $18.9 \pm 2.8$ & $4.5 \pm 0.5$ & nd \\
\hline & $\begin{array}{l}\text { L. cardiaca } \\
25 \mu \mathrm{g} . \mathrm{mg} \text { protein }{ }^{-1}\end{array}$ & $4.6 \pm 0.7$ & $18.6 \pm 3.0$ & $4.5 \pm 0.4$ & nd \\
\hline & Succinate & & & & \\
\hline & Control & $6.3 \pm 0.3$ & $25.0 \pm 1.1$ & $5.5 \pm 0.2$ & $36.6 \pm 0.9$ \\
\hline & $\begin{array}{l}\text { L. cardiaca } \\
15 \mu \mathrm{g} . \mathrm{mg} \mathrm{protein}^{-1}\end{array}$ & $7.2 \pm 0.2$ & $22.3 \pm 1.1$ & $5.9 \pm 0.2$ & $35.7 \pm 0.3$ \\
\hline & $\begin{array}{l}\text { L. cardiaca } \\
25 \mu \mathrm{g} . \mathrm{mg}^{-} \text {protein }^{-1}\end{array}$ & $7.1 \pm 0.4$ & $20.3 \pm 1.1^{*}$ & $6.4 \pm 0.2^{*}$ & $31.7 \pm 1.6^{\star *}$ \\
\hline \multicolumn{6}{|c|}{$\begin{array}{l}\text { Effects of the purified ethanolic extract of PEEMa and PEELC on rat liver mitochondrial respiratory } \\
\text { rates: } V_{3}-\text { state } 3 \text { respiratory rate; } V_{4}-\text { state } 4 \text { respiratory rate; } V_{F C C P}-\text { respiratory rate evaluate in } \\
\text { the presence of FCCP. Mitochondria }(1 \mathrm{mg}) \text { were incubated with PEEMa and PEELc for } 5 \text { min in } 1 \\
\mathrm{~mL} \text { of the standard respiration medium at } 30^{\circ} \mathrm{C} \text {, accordingly to Materials and Methods section. Data } \\
\text { were obtained with triplicates of four different mitochondrial preparations. Statistics: }{ }^{*} \mathrm{p}<0.05 \text {; }{ }^{*} \\
\mathrm{p}<0.01 \text { compared to control. }\end{array}$} \\
\hline
\end{tabular}


The concomitant decrease in state 3 (in glutamate/malate for PEEMa and succinatestimulated mitochondria in the presence of both plant extracts) followed by an increased state 4 (in succinate-stimulated mitochondria in the presence of PEELc) resulted in the expected decrease in respiratory control ratio (RCR), i.e., the state 3 to state 4 ratio, in all these experimental conditions (Fig. 2A and $2 \mathrm{C}$ ). Nevertheless, there were no significant changes in the $\mathrm{ADP} / \mathrm{O}$ ratio (Fig. $2 \mathrm{~B}$ and $2 \mathrm{D}$ ), indicating that the mitochondria phosphorylative system efficiency was not affected.
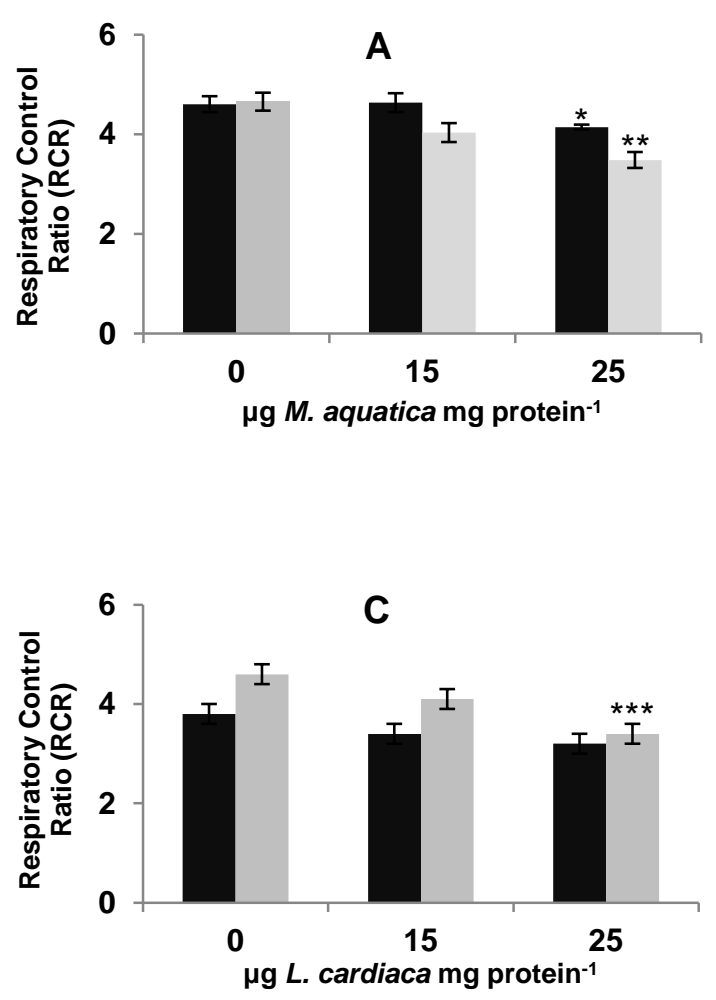

- Glut+Mal Succ

B

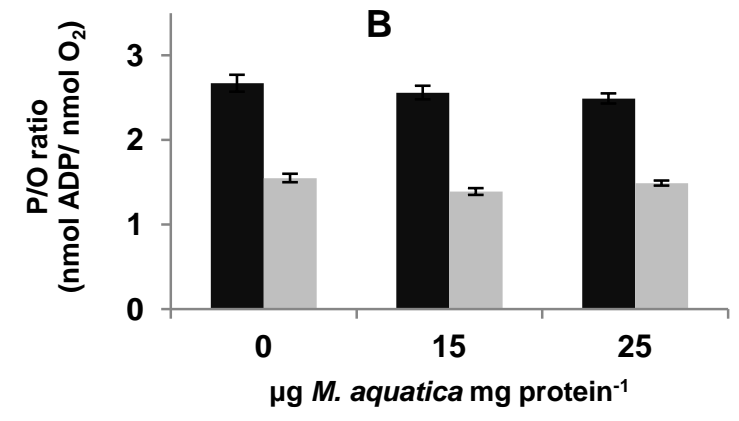

D

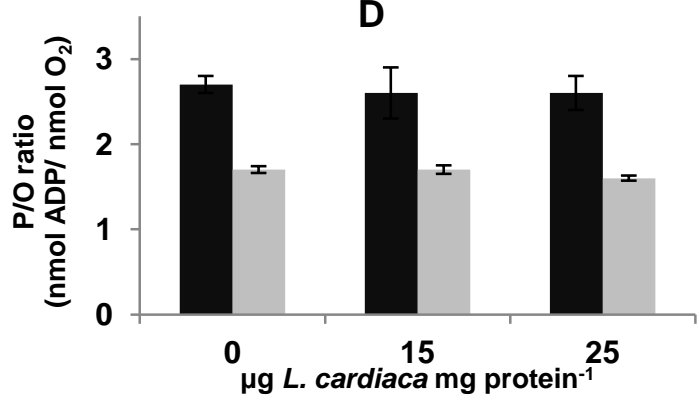

Figure 2 - Effects of the purified ethanolic extract of $M$. aquatica (PEEMa) and L. cardiaca (PEELC) on liver mitochondria respiratory indexes: respiratory control ratio (RCR) $(A, C)$ and $\mathrm{P} / \mathrm{O}$ ratio $(\mathrm{B}, \mathrm{D})$. Mitochondria (1 $\mathrm{mg}$ protein) were incubated in $1 \mathrm{~mL}$ respiratory standard medium containing glutamate + malate $(5 \mathrm{mM}+5 \mathrm{mM})$ or succinate $(5 \mathrm{mM})+$ rotenone $(1 \mu \mathrm{M})$. State 3 respiration was initiated by the addition of $125 \mathrm{nmol}$ ADP. Values are the means \pm SEM of triplicates performed with 4 different mitochondrial preparations. Statistics: ${ }^{*} p<0.05 ;{ }^{* *} p<$ $0.01 ;{ }^{* * *} p<0.001$ as compared to control.

The RCR is the most useful general measurement of mitochondria fitness, because of its influence by almost every functional aspect of oxidative phosphorylation. Indeed, respiratory state 3 is controlled approximately in equal mode (depending on the tissue 
and conditions) by the phosphorylative system activity (primarily the adenine nucleotide translocase, phosphate transporter and ATP synthase) and substrate oxidation (including substrate uptake, processing enzymes, relevant electron-transport chain complexes, pool sizes of $U Q$ (ubiquinone) and cytochrome $c$, and $\left[\mathrm{O}_{2}\right]$ ). Therefore, inhibition of any of these processes will decrease the state 3 respiratory rate [1] and consequently, the RCR. In turn, respiratory state 4 (and RCR) is highly influenced by proton leak $[1,20]$.

The results herein obtained showed that, with the exception of PEELc in the presence of glutamate/malate respiratory substrate, the remaining conditions (succinate for PEELC), as well as PEEMa (both respiratory substrates) decreased RCR. This was due to a decrease in respiratory state 3 (PEEMa), while PEELc affected both the respiratory states 3 and 4 .

\section{Effects of PEEMa and PEELc on oxidative phosphorylation}

Alterations in oxidative phosphorylation can also be accurately evaluated through monitoring of membrane potential fluctuations with a TPP ${ }^{+}$-selective electrode [19]. As shown in Table 2, under control conditions, mitochondria developed a $\Delta \Psi$ after substrate addition of approximately $-210 \mathrm{mV}$ in glutamate/malate and close to -218 $\mathrm{mV}$ in succinate-sustained respiration. Upon ADP addition, there was an expected drop in the membrane potential (27-28 $\mathrm{mV}$ or 29-32 $\mathrm{mV}$ for glutamate/malate or succinatestimulated respiration, respectively), as ATP synthase uses $\Delta \Psi$ to phosphorylate the ADP during state 3 respiration. This is followed by a $\Delta \Psi$ repolarization, which occurs after a short lag phase taking place along ADP phosphorylation (Fig. 1). 
Table 2 - Effects of the M. aquatica (PEEMa) and L. cardiaca (PEELC) purified ethanolic extracts on rat liver mitochondria membrane potential.

\begin{tabular}{|c|c|c|c|c|c|c|}
\hline $\begin{array}{l}\text { Plant } \\
\text { extract }\end{array}$ & Condition & $\begin{array}{l}\text { Energization } \\
\qquad(\mathrm{mV})\end{array}$ & $\begin{array}{c}\Delta \text { ADP1 } \\
(\mathrm{mV})\end{array}$ & $\begin{array}{l}\text { Rep } \\
(\mathrm{mV})\end{array}$ & $\begin{array}{c}\text { Vrep } \\
\text { (\% of mean } \\
\text { control) }\end{array}$ & $\begin{array}{c}\text { Lag phase } \\
\text { (s) }\end{array}$ \\
\hline \multirow{8}{*}{ PEEMa } & Glutamate + Malate & & & & & \\
\hline & Control & $209.3 \pm 1.5$ & $26.9 \pm 2.0$ & $205.7 \pm 1.2$ & $100.0 \pm 6.0$ & $46.7 \pm 1.2$ \\
\hline & $\begin{array}{l}\text { M. aquatica } \\
15 \mu \mathrm{g} \cdot \mathrm{mg} \mathrm{protein}^{-1}\end{array}$ & $202.9 \pm 2.3$ & $27.1 \pm 2.1$ & $200.3 \pm 2.4^{*}$ & $84.8 \pm 5.0$ & $55.5 \pm 1.0^{*}$ \\
\hline & 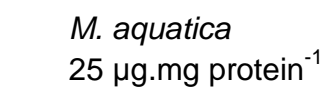 & $200.8 \pm 2.4^{*}$ & $25.8 \pm 1.4$ & $199.9 \pm 1.3^{*}$ & $75.5 \pm 5.0^{*}$ & $65.5 \pm 3.2^{\star *}$ \\
\hline & Succinate & & & & & \\
\hline & Control & $218.8 \pm 1.0$ & $29.0 \pm 1.1$ & $218.4 \pm 1.1$ & $100.0 \pm 5.7$ & $66.6 \pm 5.1$ \\
\hline & $\begin{array}{l}\text { M. aquatica } \\
15 \mu \mathrm{g} . \mathrm{mg} \mathrm{protein}^{-1}\end{array}$ & $218.3 \pm 0.9$ & $28.5 \pm 2.4$ & $216.7 \pm 1.6$ & $91.4 \pm 4.3$ & $64.0 \pm 6.6$ \\
\hline & $\begin{array}{l}\text { M. aquatica } \\
25 \mu \mathrm{g} \cdot \mathrm{mg} \mathrm{protein}^{-1}\end{array}$ & $215.0 \pm 1.2^{*}$ & $27.9 \pm 1.3$ & $214.1 \pm 1.4^{*}$ & $75.7 \pm 3.4^{\star *}$ & $76.6 \pm 5.0^{*}$ \\
\hline \multirow{8}{*}{ PEELc } & Glutamate + Malate & & & & & \\
\hline & Control & $212.4 \pm 1.6$ & $28.4 \pm 0.8$ & $210.7 \pm 1.6$ & $100.0 \pm 4.3$ & $55 \pm 2.7$ \\
\hline & $\begin{array}{l}\text { L. cardiaca } \\
15 \mu \mathrm{g} . \mathrm{mg} \mathrm{protein}^{-1}\end{array}$ & $208.1 \pm 1.7$ & $27.9 \pm 1.1$ & $205.6 \pm 1.7$ & $104.0 \pm 3.7$ & $53.8 \pm 4.8$ \\
\hline & $\begin{array}{l}\text { L. cardiaca } \\
25 \mu \mathrm{g} \cdot \mathrm{mg} \mathrm{protein}^{-1}\end{array}$ & $206.5 \pm 2.1$ & $24.3 \pm 0.7^{*}$ & $204.4 \pm 2.1^{*}$ & $82.8 \pm 4.2^{*}$ & $53.2 \pm 2.0$ \\
\hline & Succinate & & & & & \\
\hline & Control & $217.1 \pm 1.1$ & $32.6 \pm 1.0$ & $217.0 \pm 1.1$ & $100.0 \pm 4.1$ & $58.1 \pm 1.6$ \\
\hline & $\begin{array}{l}\text { L. cardiaca } \\
15 \mu \mathrm{g} . \mathrm{mg}^{-1} \text { protein }^{-1}\end{array}$ & $213.2 \pm 1.0^{*}$ & $35.4 \pm 1.0$ & $212.2 \pm 1.0^{*}$ & $86.1 \pm 3.5^{\star}$ & $72.6 \pm 2.0^{\star *}$ \\
\hline & $\begin{array}{l}\text { L. cardiaca } \\
25 \mu \mathrm{g} . \mathrm{mg} \mathrm{protein}^{-1}\end{array}$ & $212.8 \pm 0.8^{*}$ & $28.3 \pm 1.2^{*}$ & $211.3 \pm 0.9^{\star *}$ & $68.6 \pm 3.4^{\star \star \star}$ & $81.0 \pm 3.7^{\star \star *}$ \\
\hline
\end{tabular}

Mitochondria $(1 \mathrm{mg})$ were incubated with plant extract for $5 \mathrm{~min}$ in $1 \mathrm{~mL}$ of the standard respiration medium supplemented with $3 \mu \mathrm{M} \mathrm{TPP}+$ at $30^{\circ} \mathrm{C}$, accordingly to Materials and Methods section. Statistics: ${ }^{*} \mathrm{p}<0.05$; ${ }^{* *} p<0.01 ;{ }^{* * *} p<0.001$ as compared to control.

The treatment of mitochondria with increasing concentrations of PEEMa and PEELC (15 and $25 \mu \mathrm{g} . \mathrm{mg} \mathrm{protein}^{-1}$ ) resulted in a progressive decrease of $\Delta \Psi$ max, regardless of the respiratory substrate used (Table 2). Statistical differences were observed for 25 $\mu$ g.mg protein $^{-1}$ of PEEMa, for both glutamate/malate and succinate substrates, whereas the effect of PEELc was particularly evident for succinate-induced 
polarization. For the highest concentration tested, this extract also caused a significant decrease in the depolarization amplitude following ADP addition, for both substrates.

Additionally, the results also showed that PEEMa and PEELc impaired $\Delta \Psi$ total repolarization, i.e, after ADP addition, mitochondria in the presence of the plant extracts, were never able to recover $\Delta \Psi$ to control values. With the exception of PEELc in glutamate/malate sustained respiration, there was also a consistent increase in the phosphorylative lag phase, i.e., the time that the membrane potential takes to recover after ADP addition (Table 2). In general, this effect is in agreement with the decrease of state 3 .

Obviously, it will be important to further search for potential sites of disturbance on the activity in the respiratory chain, as well as on the phosphorilative system, in order to pinpoint the main causes of bioenergetic dysfunction induced by PEEMa and PEELc. Due to the high phenolic content of these extracts $(302.5 \pm 8.0$ and $500.4 \pm 49.1$, for PEEMa and PEELc respectively), it is possible that phenolic constituents are active players in this process. Note that, due to their amphiphilicity, these are able to be inserted in mitochondrial inner membrane, affecting their lipidic domains structure and fluidity [21, 22]. Also important, the partial lowering of mitochondrial membrane potential might have an important physiological meaning, since according to that previously demonstrated by Korshunov et al. [23], a small decrease in the mitochondrial $\Delta \Psi$ is responsible for a large decrease in the production of reactive oxygen species by the respiratory chain, a phenomenon called "mild uncoupling". The mild mitochondrial stress induced by the polyphenols present in PEEMa, that act as hormetic stimuli, can account for the antioxidant and anti-inflammatory properties of $M$. aquatica observed in vivo [24] and contribute also to a higher mitochondrial flexibility [25]. These hormetic stimuli can also be important in the prevention of other chronic pathologies, related with oxidative stress and the human modern nutritional milieu [25].

\subsubsection{References}

[1] Brand, M. D.; Nicholls, D. G., Assessing mitochondrial dysfunction in cells. Biochemical Journal, 2011, 435, 297-312.

[2] Tait, S. W. G.; Green, D. R., Mitochondria and cell signalling. Journal of Cell Science, 2012, 125(4), 807-815.

[3] Labbe, G.; Pessayre, D.; Fromenty, B., Drug-induced liver injury through mitochondrial dysfunction: mechanisms and detection during preclinical safety studies. Fundamental \& Clinical Pharmacology, 2008, 22(4), 335-353. 
[4] Dykens, J. A.; Will, Y., The significance of mitochondrial toxicity testing in drug development. Drug Discovery Today, 2007, 12(17-18), 777-785.

[5] Szewczyk, A.; Wojtczak, L., Mitochondria as a Pharmacological Target. Pharmacological Reviews, 2002, 54(1), 101-127.

[6] McKim, J. M., Jr., Building a Tiered Approach to In Vitro Predictive Toxicity Screening: A Focus on Assays with In Vivo Relevance. Combinatorial Chemistry \& High Throughput Screening, 2010, 13(2), 188-206.

[7] Scatena, R.; Bottoni, P.; Botta, G.; Martorana, G. E.; Giardina, B., The role of mitochondria in pharmacotoxicology: a reevaluation of an old, newly emerging topic. American Journal of Physiology-Cell Physiology, 2007, 293(1), C12-C21.

[8] Choumessi, A. T.; Loureiro, R.; Silva, A. M.; Moreira, A. C.; Pieme, A. C.; Tazoacha, A.; Oliveira, P. J.; Penlap, V. r. B., Toxicity evaluation of some traditional African spices on breast cancer cells and isolated rat hepatic mitochondria. Food and Chemical Toxicology, 2012, 50(11), 4199-4208.

[9] Gella, E. V.; Pasechnik, I. K. H. Prodn. of flavonoid(s) for use as bile secretion stimulants - from Mentha aquatica $L$ plant tissue, includes extraction with methanol after chloroform treatment to increase yield. SU603388-A, SU603388-A 21 Mar 1978 197910.

[10] Blasius, W. Herbal extract compsn. for bathing hands and feet - to treat chronic diseases e.g. migraine, rheumatism, asthma, eczema, gout, arthritis and angina pectoris. DE1692045-A1; DE1692045-A, DE1692045-A1 DE1692045-A 06 May 1971 198501 Pages: 12.

[11] Zhang, H.; Liu, X. Composition useful e.g. for treating dysmenorrhea, tonifying liver and kidney, and relieving pain, comprises e.g. Chinese Angelica, radix Paeonia alba, rhizoma Sparganium, Curcuma zedoaria, peach kernel, Leonurus and liquorice. CN102240383-A, CN102240383-A 16 Nov 2011 A61K-036/9066 201221, 2011.

[12] EMEA, Assessment report on Leonurus cardiaca L., herba 2010.

[13] Pereira, O. R.; Domingues, M. R. M.; Silva, A. M. S.; Cardoso, S. M., Phenolic constituents of Lamium album: Focus on isoscutellarein derivatives. Food Research International, 2012, 48(1), 330-335.

[14] Gazotti, P.; Malmstron, K.; Crompton, M., Membrane Biochemistry. SpringerVerlag: New York, 1979.

[15] Ferreira, F. M.; Dinis, L. T.; Azedo, P.; Galhano, C. I. C.; Simoes, A.; Cardoso, S. M.; Domingues, M. R. M.; Pereira, O. R.; Palmeira, C. M.; Peixoto, F. P., Antioxidant capacity and toxicological evaluation of Pterospartum tridentatum flower extracts. CytaJournal of Food, 2012, 10(2), 92-102.

[16] Monteiro, J. P.; Oliveira, P. J.; Moreno, A. J. M.; Jurado, A. S., Disruption of hepatic mitochondrial bioenergetics is not a primary mechanism for the toxicity of methoprene - Relevance for toxicological assessment. Chemosphere, 2008, 72(9), 1347-1354.

[17] Gornall, A. G.; Bardawill, C. J.; David, M. M., Determination of serum proteins by means of the biuret reaction. Journal of Biological Chemistry, 1949, 177(2), 751-766. 
[18] Chance, B.; Williams, G. R., The respiratory chain and oxidative phosphorylation Advances in Enzymology and Related Subjects of Biochemistry, 1956, 17, 65-134.

[19] Ferreira, F. M.; Palmeira, C. M.; Seica, R.; Moreno, A. J.; Santos, M. S., Diabetes and mitochondrial bioenergetics: Alterations with age. Journal of Biochemical and Molecular Toxicology, 2003, 17(4), 214-222.

[20] Aidt, F. H.; Nielsen, S. M. B.; Kanters, J.; Pesta, D.; Nielsen, T. T.; Norremolle, A.; Hasholt, L.; Christiansen, M.; Hagen, C. M., Dysfunctional mitochondrial respiration in the striatum of the Huntington's disease transgenic R6/2 mouse model. PLoS currents, 2013, 5 .

[21] Bonarska-Kujawa, D.; Pruchnik, H.; Oszmianski, J.; Sarapuk, J.; Kleszczynska, H., Changes Caused by Fruit Extracts in the Lipid Phase of Biological and Model Membranes. Food Biophysics, 2011, 6(1), 58-67.

[22] Krishnan, R.; Maru, G. B., Isolation and analyses of polymeric polyphenol fractions from black tea. Food Chemistry, 2006, 94(3), 331-340.

[23] Korshunov, S. S.; Skulachev, V. P.; Starkov, A. A., High protonic potential actuates a mechanism of production of reactive oxygen species in mitochondria. Febs Letters, 1997, 416(1), 15-18.

[24] Conforti, F.; Sosa, S.; Marrelli, M.; Menichini, F.; Statti, G. A.; Uzunov, D.; Tubaro, A.; Menichini, F.; Della Loggia, R., In vivo anti-inflammatory and in vitro antioxidant activities of Mediterranean dietary plants. Journal of Ethnopharmacology, 2008, 116(1), 144-151.

[25] Nunn, A. V. W.; Bell, J. D.; Guy, G. W., Lifestyle-induced metabolic inflexibility and accelerated ageing syndrome: insulin resistance, friend or foe? Nutrition \& Metabolism, 2009, 6 . 


\section{CONCLUSIONS}




\section{FIRST CONCLUSION}

HPLC-DAD combined with ESI-MS ${ }^{n}$ in the negative mode plus NMR spectroscopy are valuable analytical techniques for phenolic characterization of ethanolic extracts obtained from Cytisus multiflorus, Lamium album L., Lavandula dentata L., Leonurus cardiaca L., Mentha aquatica L. and Thymus x citriodorus.

\section{SECOND CONCLUSION}

The plant species in focus in the present work have distinct phenolic profiles. While $C$. multiflorus and T. $x$ citriodorus are enriched in flavones, M. aquatica contains high amounts of flavanones (eriodictyol, naringenin and hesperitin glycosides). In turn, $L$. dentata almost exclusively contains rosmarinic acid and $L$. album and L. cardiaca mainly contain phenylethanoid glycosides.

\section{THIRD CONCLUSION}

As determined by two chemical assays, namely DPPH scavenging and reducing power assays, the six plant extracts possess high antioxidant activity. The four most relevant antioxidant extracts show the potency order of $M$. aquatica $>L$. album $>L$. dentata $>T$. $x$ citriodorus and overall the $\mathrm{EC}_{50}$ values of the six plant extracts ranged from 8.1 to $18.3 \mu \mathrm{g} / \mathrm{mL}$ and 51.9 to $95.7 \mu \mathrm{g} / \mathrm{mL}$, for DPPH scavenging and reducing power assays, respectively.

\section{FOURTH CONCLUSION}

The extracts obtained from C. multiflorus, L. album, T. $x$ citriodorus and L. dentata medicinal plants effectively counteract the increased ROS formation in oxidative stress models in liver cells, namely in a human hepatoblastoma HepG2 cells.

\section{FIFTH CONCLUSION}

L. album, C. multiflorus, T. $x$ citriodorus and M. aquatica ethanolic extracts exert cytoprotective effects in human hepatoblastoma HepG2 cells under oxidative stress conditions. 


\section{SIXTH CONCLUSION}

The ROS-scavenging and cytoprotective activities of C. multiflorus, L. album, T. $x$ citriodorus extracts in the HepG2 oxidative stress model are closely associated to their phenolic constituents.

\section{SEVENTH CONCLUSION}

C. multiflorus extract anti-inflammatory activities are mediated through $\mathrm{NO}^{\bullet}$ radical scavenging and decrement of the expression of the enzyme iNOS, as well as inhibition of 5-LOX activity.

\section{EIGHTH CONCLUSION}

Liver mitochondria fitness is affected by $M$. aquatica and $L$. cardiaca extracts. $M$. aquatica extract induce a decrease in respiratory state 3 , while $L$. cardiaca extract affect the respiratory states 3 and 4 . Both plant extracts decrease the respiratory control ratio without affecting the phosphorilative efficiency of mitochondria.

\section{GLOBAL CONCLUSION}

C. multiflorus, L. album, L. dentata, L. cardiaca, M. aquatica and T. $x$ citriodorus are good sources of phenolic compounds. Having in mind the physiopathologic role of oxidative stress in several diseases, together with the high antioxidant capacity shown for the majority of the plant extracts herein in focus, it if feasible to propose them as potential preventive agents. Additionally, C. multiflorus seems to be a potential agent in inflammation-related disorders. 
5. REFERENCES 
[1] Manach, C.; Scalbert, A.; Morand, C.; Remesy, C.; Jimenez, L., Polyphenols: food sources and bioavailability. The American Journal of Clinical Nutrition, 2004, 79(5), 727-747.

[2] Cristofolini, G.; Conte, L., Phylogenetic patterns and endemism genesis in Cytisus Desf. (Leguminosae-Cytiseae) and related genera. Israel Journal of Plant Sciences, 2002, 50, 37-50.

[3] Cristofolini, G.; Troia, A., A reassessment of the sections of the genus Cytisus Desf. (Cytiseae, Leguminosae). Taxon, 2006, 55(3), 733-746.

[4] Raja, S.; Ahamed, K.; Kumar, V.; Mukherjee, K.; Bandyopadhyay, A.; Mukherjee, P. K., Antioxidant effect of Cytisus scoparius against carbon tetrachloride treated liver injury in rats. Journal of Ethnopharmacology, 2007, 109(1), 41-47.

[5] Sundararajan, R.; Haja, N. A.; Venkatesan, K.; Mukherjee, K.; Saha, B. P.; Bandyopadhyay, A.; Mukherjee, P. K., Cytisus scoparius link-a natural antioxidant. BMC Complementary and Alternative Medicine, 2006, 6, 8.

[6] Nirmal, J.; Babu, C. S.; Harisudhan, T.; Ramanathan, M., Evaluation of behavioural and antioxidant activity of Cytisus scoparius Link in rats exposed to chronic unpredictable mild stress. BMC Complementary and Alternative Medicine, 2008, 8, 8.

[7] Siegel, R. K., Herbal intoxication- Psychoactive effects from herbal cigarettes, tea, and capsules. Jama-Journal of the American Medical Association, 1976, 236(5), 473476.

[8] Di Giorgio, C.; Delmas, F.; Tueni, M.; Cheble, E.; Khalil, T.; Balansard, G., Alternative and complementary antileishmanial treatments: Assessment of the antileishmanial activity of 27 Lebanese plants, including 11 endemic species. Journal of Alternative and Complementary Medicine, 2008, 14(2), 157-162.

[9] Castro, V., Chromium in a series of Portuguese plants used in the herbal treatment of diabetes. Biological Trace Element Research, 1998, 62(1-2), 101-106.

[10] Castro, V., Chromium and zinc in a series of plants used in Portugal in the herbal treatment of non-insulinized diabetes. Acta Alimentaria, 2001, 30(4), 333-342.

[11] Rodriguez-Riano, T.; Ortega-Olivencia, A.; Devesa, J. A., Reproductive biology in Cytisus multiflorus (Fabaceae). Annales Botanici Fennici, 2004, 41(3), 179-188.

[12] Ciudad, A. G.; Santos, B. F.; de Aldana, B. R. V.; Zabalgogeazcoa, I.; Gutierrez, M. Y.; Criado, B. G., Use of near infrared reflectance spectroscopy to assess foragequality of a Mediterranean shrub. Communications in Soil Science and Plant Analysis, 2004, 35(5-6), 665-678.

[13] Rodriguez-Riano, T.; Valtuena, F. J.; Ortega-Olivencia, A., Megasporogenesis, megagametogenesis and ontogeny of the aril in Cytisus striatus and C-multiflorus (Leguminosae : Papilionoideae). Annals of Botany, 2006, 98(4), 777-791.

[14] Rodriguez-Riano, T.; Ortega-Olivencia, A.; Devesa, J. A., Reproductive phenology in three Genisteae (Fabaceae) shrub species of the W Mediterranean Region. Nordic Journal of Botany, 1999, 19(3), 345-354.

[15] Gião, M. S.; Gonzalez-Sanjose, M. L.; Rivero-Perez, M. D.; Pereira, C. I.; Pintado, M. E.; Malcata, F. X., Infusions of Portuguese medicinal plants: Dependence of final 
antioxidant capacity and phenol content on extraction features. Journal of the Science of Food and Agriculture, 2007, 87(14), 2638-2647.

[16] Barros, L.; Duenas, M.; Carvalho, A. M.; Ferreira, I. C. F. R.; Santos-Buelga, C., Characterization of phenolic compounds in flowers of wild medicinal plants from Northeastern Portugal. Food and Chemical Toxicology, 2012, 50(5), 1576-82.

[17] Yalcin, F. N.; Kaya, D., Ethnobotany, Pharmacology and Phytochemistry of the Genus Lamium (Lamiaceae). FABAD Journal of Pharmaceutical Sciences, 2006, 31, 43-52.

[18] Matkowski, A.; Piotrowska, M., Antioxidant and free radical scavenging activities of some medicinal plants from the Lamiaceae. Fitoterapia, 2006, 77(5), 346-353.

[19] Akkol, E. K.; Yalcin, F. N.; Kaya, D.; Calis, I.; Yesilada, E.; Ersoz, T., In vivo antiinflammatory and antinociceptive actions of some Lamium species. Journal of Ethnopharmacology, 2008, 118(1), 166-172.

[20] Heinrich, M.; Müller, W. E.; Galli, C., Local Mediterranean Food Plants and Nutraceuticals. Forum Nutr. Basel, Karger: 2006; Vol. 59.

[21] Clifford, A. W., Mediterranean Vegetables: A Cook's ABC of Vegetables and Their Preparation. The Harvard Common Press: Boston, Massachusetts, 2001.

[22] Celtnet Celtnet Recipes: Wild Food Guide For White Deadnettle (Lamium album). $\leq$ http://www.celtnet.org.uk/recipes/ancient/wild-food-

entry.php?term=White\%20Deadnettle $>$ (accessed 26-04-2012).

[23] Harford, R. Eat Weeds: Wild food guide to the edible plants of Britain $<$ http://www.eatweeds.co.uk/> (accessed 26-06-2012).

[24] Denisow, B.; Bozek, M., Blooming and pollen production of two Lamium L. species. Journal of Apicultural Science, 2008, 52(1), 21-30.

[25] Mihaly Cozmuta, A.; Bretan, L.; Mihaly Cozmuta, L.; Nicula, C.; Peter, A., Lead traceability along soil-melliferrous flora-bee family-apiary products chain. Journal of Environmental Monitoring, 2012, Accepted manuscript.

[26] Paduch, R.; Wójciak-Kosior, M.; Matysik, G., Investigation of biological activity of Lamii albi flos extracts. Journal of Ethnopharmacology, 2007, 110(1), 69-75.

[27] Paduch, R.; Matysik, G.; Wojciak-Kosior, M.; Kandefer-Szerszen, M.; SkalskaKaminska, A.; Nowak-Kryska, M.; Niedziela, P., Lamium Album extracts express free radical scavenging and cytotoxic activities. Polish Journal of Environmental Studies, 2008, 17(4), 569-580.

[28] Xu, F. Chinese medicine e.g. for treating arthropathy, comprises broad cocklebur, vervain, condyle grass, motherwort, saxifrage, cactus, mulberry branch, white dead nettle, Boston ivy, Folium photiniae, water pepper and Chinese fevervine. CN101164604-A; CN101164604-B, CN101164604-A 23 Apr 2008 A61K-036/9062 200868, 2008.

[29] Ninomiya, K.; Nishida, S.; Matsura, Y.; Asada, M.; Kawahara, Y.; Yoshikawa, M.; Nishida, N.; Matsuura, Y. Fat-metabolism improving agent for use in food/drink for improving fat metabolism and preventing/treating lifestyle related disease e.g. diabetes, contains polar solvent extract of herb e.g. rose hip fruit, mugwort or safflower.

Pereira O. R., 2013 | CHARACTERIZATION OF PHENOLIC CONSTITUENTS OF MEDICINAL PLANTS AND EVALUATION OF PHARMACOLOGICAL ACTIVITIES: FOCUS IN ANTIOXIDANT AND 
JP2006016312-A; JP3790767-B2; US2008003312-A1, JP2006016312-A 19 Jan 2006 A61K-036/18 200610, 2006.

[30] Upson, T. M.; J. Grayer, R. e.; Greenham, J. R.; A. Williams, C.; Al-Ghamdi, F.; Chen, F.-H., Leaf flavonoids as systematic characters in the genera Lavandula and Sabaudia. Biochemical Systematics and Ecology, 2000, 28(10), 991-1007.

[31] Guyot-Declerck, C.; Renson, S.; Bouseta, A.; Collin, S., Floral quality and discrimination of Lavandula stoechas, Lavandula angustifolia, and Lavandula angustifolia x Latifolia honeys. Food Chemistry, 2002, 79(4), 453-459.

[32] Nitzsche, A.; Tokalov, S. V.; Gutzeit, H. O.; Ludwig-Muller, J., Chemical and biological characterization of cinnamic acid derivatives from cell cultures of lavender (Lavandula officinalis) induced by stress and jasmonic acid. Journal of Agricultural and Food Chemistry, 2004, 52(10), 2915-2923.

[33] Castro-Vazquez, L.; Diaz-Maroto, M. C.; Gonzalez-Vinas, M. A.; Perez-Coello, M. S., Differentiation of monofloral citrus, rosemary, eucalyptus, lavender, thyme and heather honeys based on volatile composition and sensory descriptive analysis. Food Chemistry, 2009, 112(4), 1022-1030.

[34] Ciobanu, A.; Mallard, I.; Landy, D.; Brabie, G.; Nistor, D.; Fourmentin, S., Inclusion interactions of cyclodextrins and crosslinked cyclodextrin polymers with linalool and camphor in Lavandula angustifolia essential oil. Carbohydrate Polymers, 2012, 87(3), 1963-1970.

[35] NIH Lavender. http://www.nlm.nih.gov/medlineplus/druginfo/natural/838.html (accessed 20-03-2012).

[36] Kim, N.-S.; Lee, D.-S., Comparison of different extraction methods for the analysis of fragrances from Lavandula species by gas chromatography mass spectrometry. Journal of Chromatography A, 2002, 982(1), 31-47.

[37] Dwyer, A. V.; Whitten, D. L.; Hawrelak, J. A., Herbal Medicines, other than St. John's Wort, in the Treatment of Depression: A Systematic Review. Alternative Medicine Review, 2011, 16(1), 40-49.

[38] Gamez, M. J.; Zarzuelo, A.; Risco, S.; Utrilla, P.; Jimenez, J., Hypoglycemic activity in various species of the genus Lavandula. Pharmazie, 1988, 43(6), 441-442.

[39] Khalil, A. M.; Ashy, M. A.; Eltawil, B. A. H.; Tawfiq, N. I., Constituents of local plants .5. Coumarin and triterpenoid constituents of Lavandula-dentata L. plant. Pharmazie, 1979, 34(9), 564-565.

[40] RHS The Royal Horticultural Society 2012. http://apps.rhs.org.uk/plantselector/plant?plantid=1129 (accessed 26-04-2012).

[41] Machado, M. P.; da Silva, A. L. L.; Biasi, L. A., Effect of plant growth regulators on in vitro regeneration of Lavandula dentata L. shoot tips. Journal of Biotechnology and Biodiversity, 2011, 2(3), 28-31.

[42] Sudria, C.; Pinol, M. T.; Palazon, J.; Cusido, R. M.; Vila, R.; Morales, C.; Bonfill, M.; Canigueral, S., Influence of plant growth regulators on the growth and essential oil content of cultured Lavandula dentata plantlets. Plant Cell Tissue and Organ Culture, 1999, 58(3), 177-184. 
[43] Al-Musayeib, N. M.; Mothana, R. A.; Matheeussen, A.; Cos, P.; Maes, L., In vitro antiplasmodial, antileishmanial and antitrypanosomal activities of selected medicinal plants used in the traditional Arabian Peninsular region. BMC Complementary and Alternative Medicine, 2012, 49.

[44] Yang, Z. Y.; Pan, S. L.; Huo, K. K.; Wu, B. Y.; Chao, Z., Molecular Analysis of Leonurus Species in China Based on ITS and matK Sequences. American Journal of Chinese Medicine, 2011, 39(2), 411-422.

[45] Bailey, L. H., Hortus Third: A Concise Dictionary of Plants Cultivated in the United States and Canada. Macmillan Publishing Co., Inc.: New York NY, 1976.

[46] Chen, Z.; Xiong, A.; Yin, L.; Yin, Y. Chinese medicine used for treating, e.g. obstruction of oviduct, made of raw material wine consisting of radix Salviae miltiorrhizae, Leonurus japonicas, vinegar, Chinese herbal medicine, radix Paeoniae Alba, and red paeonia. CN102274470-A, CN102274470-A 14 Dec 2011 A61K036/9066 201221, 2011.

[47] Zhang, H.; Liu, X. Composition useful e.g. for treating dysmenorrhea, tonifying liver and kidney, and relieving pain, comprises e.g. Chinese Angelica, radix Paeonia alba, rhizoma Sparganium, Curcuma zedoaria, peach kernel, Leonurus and liquorice. CN102240383-A, CN102240383-A 16 Nov 2011 A61K-036/9066 201221, 2011.

[48] Ye, J. Paste used for nourishing blood and invigorating blood circulation, contains Arillus longan, Astragalus mongholicus, Angelica sinensis, Leonurus, Eucommia ulmoides, tortoise plastron, Scutellaria baicalensis and red ginseng. CN102178776-A, CN102178776-A 14 Sep 2011 A61K-036/77 201174.

[49] Facciola, S., Cornucopia - A Source Book of Edible Plants. Kampong Publications: Vista, CA, USA, 1990.

[50] Matkowski, A.; Tasarz, P.; Szypula, E., Antioxidant activity of herb extracts from five medicinal plants from Lamiaceae, subfamily Lamioideae. Journal of Medicinal Plants Research, 2008, 2(11), 321-330.

[51] Jafari, S.; Moradi, A.; Salaritabar, A.; Hadjiakhoondi, A.; Khanavi, M., Determination of total phenolic and flavonoid contents of Leonurus cardiaca L. in compare with antioxidant activity. Research Journal of Biological Sciences, 2010, 5(7), 484-487.

[52] Xin-Hua, L.; Hong, X.; Yi-Zhun, Z., More than a "mother-benefiting" herb: cardioprotective effect of Herba leonuri. Shengli Xuebao, 2007, 59(5), 578-584.

[53] Ebrahimzadeh, M. A.; Nabavi, S. F.; Nabavi, S. M.; Eslami, B.; Asgarirad, H., In vitro antioxidant and free radical scavenging activity of Leonurus cardiaca subsp Persicus, Grammosciadium platycarpum and Onosma demawendicum. African Journal of Biotechnology, 2010, 9(51), 8865-8871.

[54] Kartnig, T.; Gruber, A.; Menzinger, S., Flavonoid-O-glycosides from the herbs of Leonurus-cardiaca. Journal of Natural Products, 1985, 48(3), 494-494.

[55] Popescu, M. L.; Dinu, M.; Toth, O., Contributions to the pharmacognostical and phytobiological study on Leonurus cardiaca L. (Lamiaceae). Farmacia, 2009, 57(4), 424-431. 
[56] Ebrahimzadeh, M. A.; Nabavi, S. M.; Nabavi, S. F., Correlation between the in vitro iron chelating activity and poly phenol and flavonoid contents of some medicinal plants. Pakistan Journal of Biological Sciences: PJBS, 2009, 12(12), 934-8.

[57] Brickell, C.; Trevor, C., The American Horticultural Society: Encyclopedia of Plants \& Flowers. USA: DK Inc.: New York, 2002; p p. 605.

[58] Aflatuni, A.; Uusitalo, J.; Ek, S.; Hohtola, A., Variation in the amount of yield and in the extract composition between conventionally produced and micropropagated peppermint and spearmint. Journal of Essential Oil Research, 2005, 17(1), 66-70.

[59] Brickell, C.; Zuk, J. D., The American Horticultural Society: A-Z Encyclopedia of Garden Plants. USA: DK Publishing: New York, NY, 1997; p p. 668.

[60] Bhat, S.; Maheshwari, P.; Kumar, S.; Kumar, A., Mentha species: In vitro Regeneration and Genetic Transformation. Molecular Biology Today, 2002, 3(1), 1123.

[61] Dorman, H. J. D.; Kosar, M.; Kahlos, K.; Holm, Y.; Hiltunen, R., Antioxidant properties and composition of aqueous extracts from Mentha species, hybrids, varieties, and cultivars. Journal of Agricultural and Food Chemistry, 2003, 51(16), 4563-4569.

[62] Krzyzanowska, J.; Janda, B.; Pecio, L.; Stochmal, A.; Oleszek, W.; Czubacka, A.; Przybys, M.; Doroszewska, T., Determination of Polyphenols in Mentha longifolia and M. piperita Field-Grown and In Vitro Plant Samples Using UPLC-TQ-MS. Journal of Aoac International, 2011, 94(1), 43-50.

[63] Hayes, J. R.; Stavanja, M. S.; Lawrence, B. M., Mint. The Genus Mentha. CRC Press Taylor and Francis: Boca Raton, 2007.

[64] Shan, C. Use of plant spearmint for preparing medicine for treating chronic gastritis, stomach and duodenal ulcer and hemorrhagic diseases. CN1528359-A, CN1528359-A 15 Sep 2004 A61K-035/78 200482, 2004.

[65] Chan, H.; Gou, Y.; Rowlands, D.; Chung, Y.; Gao, Y.; Chan, H. C.; Rowlands, D. W.; Chung, Y. W.; Chen, X.; Luo, L.; Zhong, Y. Medicine for treating woman climacteric syndrome, comprises powders of various Chinese herbal drugs e.g. Radix Panax ginseng, Radix Angelica sinensis, Radix Astragalus membranaceus and Fructus Ligustri lucidi. WO2004050106-A1; EP1462110-A1; AU2002349482-A1; US2005123629-A1; CN1735421-A; CN1329065-C, WO2004050106-A1 17 Jun 2004 A61K-035/78 200443, 2007.

[66] Wang, J. Medicine useful for treating chloasma, contains radix bupleuri, Angelica sinensis, fried radix paeoniae alba, Ligusticum wallichii, fried large-headed atractylodes rhizome, semen coicis, stiff silkworm, lucid ganoderma and ginger. CN102284051-A, CN102284051-A 21 Dec 2011 A61K-036/9068 201224, 2011.

[67] Greaves, J.; Narasimhamoorthy, B.; Wildgen, S.; Barkely, R.; Ruden, S. Plant tissue of Mentha spicata useful in breeding techniques for producing spearmint plants used for producing rosmarinic acid useful as antioxidant, antibacterial, antiviral and antiinflammatory agent, comprises rosmarinic acid. WO2012109322-A1, WO2012109322-A1 16 Aug 2012 A01H-005/00 201256. 
[68] Zhou, W.; Zhang, L.; Bai, J. Preparation of basil seed herbal tea beverage by extracting flos lonicerae, Chrysanthemum morifolium, bamboo leaves, rhizoma phragmitis, radix astragali, Prunella vulgaris, Mentha haplocalyx, and Glycyrrhiza uralensis, and adding basil seed. CN102578341-A, CN102578341-A 18 Jul 2012 A23F-003/34 201263.

[69] Huxley, A.; Griffiths, M.; Levy, M., The New royal horticultural society dictionary of gardening. The Macmillan Press Lld, 1992, London \& Basingstoke, 1: 815

[70] Zamfirache, M.-M.; Burzo, I.; Padurariu, C.; Boz, I.; Andro, A.-R.; Badea, M. L.; Olteanu, Z.; Lamban, C.; Truta, E., Studies regarding the chemical composition of volatile oils from some spontaneous and cultivated Lamiaceae species. Scientific Annals of Alexandru loan Cuza University of lasi. New Series, Section 2. Vegetal Biology, 2010, 56(1), 43-49.

Celtnet

Recipes.

http://www.celtnet.org.uk/recipes/search.php?cat=0\&period=0\&ingredient=water + mint\& uid=dyfedcb\&meal=0\&t=3 (accessed 03-12-2012).

[72] Olsen, H. T.; Stafford, G. I.; van Staden, J.; Christensen, S. B.; Jaeger, A. K., Isolation of the MAO-inhibitor naringenin from Mentha aquatica L. Journal of Ethnopharmacology, 2008, 117(3), 500-502.

[73] Conforti, F.; Sosa, S.; Marrelli, M.; Menichini, F.; Statti, G. A.; Uzunov, D.; Tubaro, A.; Menichini, F.; Della Loggia, R., In vivo anti-inflammatory and in vitro antioxidant activities of Mediterranean dietary plants. Journal of Ethnopharmacology, 2008, 116(1), 144-151.

[74] Stafford, G. I.; Pedersen, P. D.; Jager, A. K.; Van Staden, J., Monoamine oxidase inhibition by southern African traditional medicinal plants. South African Journal of Botany, 2007, 73(3), 384-390.

[75] Reddy, M. V. B.; Angers, P.; Gosselin, A.; Arul, J., Characterization and use of essential oil from Thymus vulgaris against Botrytis cinerea and Rhizopus stolonifer in strawberry fruits. Phytochemistry, 1998, 47(8), 1515-1520.

[76] Morales, R., The history, botany and taxonomy of the genus Thymus. In: Thyme, the genus Thymus. Taylor and Francis: London, 2002; $\mathrm{p} 317$.

[77] Elisabeth Stahl-Biskup, F. S., Thyme, the genus Thymus. Taylor and Francis: London, 2002; p 317.

[78] Kayaardi, S.; Durak, F.; Kayacier, A.; Kayaardi, M., Chemical characteristics of kavurma with selected condiments. International Journal of Food Properties, 2005, 8(3), 513-520.

[79] Agha, Q.; Ahmad, S.; Islam, M.; Gill, A.; Athar, M., Growth and production potential of three exotic herbs in highlands of Balochistan, Pakistan. Journal of Medicinal Plants Research, 2011, 5(4), 543-548.

[80] Omidbaigi, R.; Sefidkon, F.; Hejazi, M., Essential oil composition of Thymus citriodorus L. cultivated in Iran. Flavour and Fragrance Journal, 2005, 20(2), 237-238. 
[81] Horvath, G.; Szabo, L. G.; Hethelyi, E.; Lemberkovics, E., Essential oil composition of three cultivated Thymus chemotypes from Hungary. Journal of Essential Oil Research, 2006, 18(3), 315-317.

[82] Figueiredo, A. C.; Barroso, J. G.; Pedro, L. G.; Salgueiro, L.; Miguel, M. G.; Faleiro, M. L., Portuguese Thymbra and Thymus Species Volatiles: Chemical Composition and Biological Activities. Current Pharmaceutical Design, 2008, 14(29), 3120-3140.

[83] Mata, A. T.; Proenca, C.; Ferreira, A. R.; Serralheiro, M. L. M.; Nogueira, J. M. F.; Araujo, M. E. M., Antioxidant and antiacetylcholinesterase activities of five plants used as Portuguese food spices. Food Chemistry, 2007, 103(3), 778-786.

[84] Pinto, E.; Pina-Vaz, C.; Salgueiro, L.; Goncalves, M. J.; Costa-de-Oliveira, S.; Cavaleiro, C.; Palmeira, A.; Rodrigues, A.; Martinez-De-Oliveira, J., Antifungal activity of the essential oil of Thymus pulegioides on Candida, Aspergillus and dermatophyte species. Journal of Medical Microbiology, 2006, 55(10), 1367-1373.

[85] Brickell, C.; Zindersley, D., A-Z Encyclopedia of Garden Plants. London, 1989.

[86] Courtin, O. Use of Thymus citriodorus extract in cosmetic composition or for preparing dermatological composition to stimulate the synthesis of elastin microfibril interface located protein, and prevent and reduce the release of skin due to pregnancy. FR2941373-A1; FR2941373-B1, FR2941373-A1 30 Jul 2010 A61K-036/53 201050, 2010 .

[87] Courtin, O. Cosmetic or dermatological composition, useful to delay or to fight against skin aging and/or the appearance of signs of skin aging e.g. wrinkles, fine lines and sagging skin, comprises an extract of Thymus citriodorus. FR2944703-A1, FR2944703-A1 29 Oct 2010 A61K-036/53 201072, 2009.

[88] Sacchetti, G.; Maietti, S.; Muzzoli, M.; Scaglianti, M.; Manfredini, S.; Radice, M.; Bruni, R., Comparative evaluation of 11 essential oils of different origin as functional antioxidants, antiradicals and antimicrobials in foods. Food Chemistry, 2005, 91(4), 621-632.

[89] Randhir, R.; Lin, Y. T.; Shetty, K., Phenolics, their antioxidant and antimicrobial activity in dark germinated fenugreek sprouts in response to peptide and phytochemical elicitors. Asia Pacific Journal of Clinical Nutrition, 2004, 13(3), 295-307.

[90] Fu, G.; Pang, H.; Wong, Y. H., Naturally Occurring Phenylethanoid Glycosides: Potential Leads for New Therapeutics. Current Medicinal Chemistry, 2008, 15(25), 2592-2613.

[91] El Gharras, H., Polyphenols: food sources, properties and applications - a review. International Journal of Food Science and Technology, 2009, 44(12), 2512-2518.

[92] Lule, S. U.; Xia, W. S., Food phenolics, pros and cons: A review. Food Reviews International, 2005, 21(4), 367-388.

[93] Reichling, J.; Nolkemper, S.; Stintzing, F. C.; Schnitzler, P., Impact of Ethanolic Lamiaceae Extracts on Herpesvirus Infectivity in Cell Culture. Forschende Komplementarmedizin, 2008, 15(6), 313-320. 
[94] Hossain, M. B.; Patras, A.; Barry-Ryan, C.; Martin-Diana, A. B.; Brunton, N. P., Application of principal component and hierarchical cluster analysis to classify different spices based on in vitro antioxidant activity and individual polyphenolic antioxidant compounds. Journal of Functional Foods, 2011, 3(3), 179-189.

[95] Shan, B.; Cai, Y. Z.; Sun, M.; Corke, H., Antioxidant capacity of 26 spice extracts and characterization of their phenolic constituents. Journal of Agricultural and Food Chemistry, 2005, 53(20), 7749-7759.

[96] Wojdylo, A.; Oszmianski, J.; Czemerys, R., Antioxidant activity and phenolic compounds in 32 selected herbs. Food Chemistry, 2007, 105(3), 940-949.

[97] Luis, A.; Domingues, F.; Duarte, A. P., Bioactive Compounds, RP-HPLC Analysis of Phenolics, and Antioxidant Activity of Some Portuguese Shrub Species Extracts. Natural Product Communications, 2011, 6(12), 1863-1872.

[98] Raja, S.; Nazeer, H.; Kumar, V.; Kakali, M.; Bishnu, S.; Arun, B.; Pulok, M., Cytisus scoparius link - A natural antioxidant. BMC Complementary and Alternative Medicine, 2006, 6(1), 1-7.

[99] Yalcin, F. N.; Kaya, D.; Calis, I.; Ersoz, T.; Palaska, E., Determination of iridoid glycosides from four Turkish Lamium species by HPLC-ESI/MS. Turkish Journal of Chemistry, 2008, 32(4), 457-467.

[100] Justesen, U., Negative atmospheric pressure chemical ionisation low-energy collision activation mass spectrometry for the characterisation of flavonoids in extracts of fresh herbs. Journal of Chromatography A, 2000, 902(2), 369-379.

[101] Zheng, W.; Wang, S. Y., Antioxidant activity and phenolic compounds in selected herbs. Journal of Agricultural and Food Chemistry, 2001, 49(11), 5165-5170.

[102] Zgorka, G.; Glowniak, K., Variation of free phenolic acids in medicinal plants belonging to the Lamiaceae family. Journal of Pharmaceutical and Biomedical Analysis, 2001, 26(1), 79-87.

[103] Guedon, D. J.; Pasquier, B. P., Analysis and distribution of flavonoid glycosides and rosmarinic acid in 40 Mentha x piperita clones. Journal of Agricultural and Food Chemistry, 1994, 42(3), 679-684.

[104] Areias, F. M.; Valentao, P.; Andrade, P. B.; Ferreres, F.; Seabra, R. M., Phenolic fingerprint of peppermint leaves. Food Chemistry, 2001, 73(3), 307-311.

[105] Wang, H.; Provan, G. J.; Helliwell, K., Determination of rosmarinic acid and caffeic acid in aromatic herbs by HPLC. Food Chemistry, 2004, 87(2), 307-311.

[106] Hossain, M. B.; Rai, D. K.; Brunton, N. P.; Martin-Diana, A. B.; Barry-Ryan, C., Characterization of Phenolic Composition in Lamiaceae Spices by LC-ESI-MS/MS. Journal of Agricultural and Food Chemistry, 2010, 58(19), 10576-10581.

[107] Inoue, T.; Sugimoto, Y.; Masuda, H.; Kamei, C., Antiallergic effect of flavonoid glycosides obtained from Mentha piperita L. Biological \& Pharmaceutical Bulletin, 2002, 25(2), 256-259.

[108] Kosar, M.; Dorman, H. J. D.; Hiltunen, R., Effect of an acid treatment on the phytochemical and antioxidant characteristics of extracts from selected Lamiaceae species. Food Chemistry, 2005, 91(3), 525-533. 
[109] Miura, K.; Nakatani, N., Antioxidative activity of flavonoids from thyme (Thymusvulgaris L). Agricultural and Biological Chemistry, 1989, 53(11), 3043-3045.

[110] Parejo, I.; Viladomat, F.; Bastida, J.; Rosas-Romero, A.; Flerlage, N.; Burillo, J.; Codina, C., Comparison between the radical scavenging activity and antioxidant activity of six distilled and nondistilled Mediterranean herbs and aromatic plants. Journal of Agricultural and Food Chemistry, 2002, 50(23), 6882-6890.

[111] Misan, A. C.; Mimica-Dukic, N. M.; Mandic, A. I.; Sakac, M. B.; Milovanovic, I. L.; Sedej, I. J., Development of a rapid resolution HPLC method for the separation and determination of 17 phenolic compounds in crude plant extracts. Central European Journal of Chemistry, 2011, 9(1), 133-142.

[112] Proestos, C.; Chorianopoulos, N.; Nychas, G. J. E.; Komaitis, M., RP-HPLC analysis of the phenolic compounds of plant extracts. Investigation of their antioxidant capacity and antimicrobial activity. Journal of Agricultural and Food Chemistry, 2005, 53(4), 1190-1195.

[113] Hanganu, D.; Vlase, L.; Olah, N., Phytochemical Analysis of Isoflavons from some Fabaceae Species Extracts. Notulae Botanicae Horti Agrobotanici Cluj-Napoca, 2010, 38(1), 57-60.

[114] Miron, T. L.; Plaza, M.; Bahrim, G.; Ibanez, E.; Herrero, M., Chemical composition of bioactive pressurized extracts of Romanian aromatic plants. Journal of Chromatography A, 2011, 1218(30), 4918-4927.

[115] Lee, C.-J.; Chen, L.-G.; Chang, T.-L.; Ke, W.-M.; Lo, Y.-F.; Wang, C.-C., The correlation between skin-care effects and phytochemical contents in Lamiaceae plants. Food Chemistry, 2011, 124(3), 833-841.

[116] Dolzhenko, Y.; Bertea, C. M.; Occhipinti, A.; Bossi, S.; Maffei, M. E., UV-B modulates the interplay between terpenoids and flavonoids in peppermint (Mentha $x$ piperita L.). Journal of Photochemistry and Photobiology B-Biology, 2010, 100(2), 6775.

[117] Kurkin, V. A.; Lamrini, M., Flavonoids of Lavandula spica flowers. Chemistry of Natural Compounds, 2007, 43(6), 702-703.

[118] Georgiev, M.; Kovacheva, E.; Marcheva, N.; Ilieva, M., Purification of rosmarinic acid extracts from Lavandula vera MM cell biomass. Food Chemistry, 2006, 94(1), 111 114.

[119] Dapkevicius, A.; van Beek, T. A.; Lelyveld, G. P.; van Veldhuizen, A.; de Groot, A.; Linssen, J. P. H.; Venskutonis, R., Isolation and structure elucidation of radical scavengers from Thymus vulgaris leaves. Journal of Natural Products, 2002, 65(6), 892-896.

[120] Fecka, I.; Turek, S., Determination of water-soluble polyphenolic compounds in commercial herbal teas from Lamiaceae: Peppermint, melissa, and sage. Journal of Agricultural and Food Chemistry, 2007, 55(26), 10908-10917.

[121] Kulisic, T.; Dragovic-Uzelac, V.; Milos, M., Antioxidant activity of aqueous tea infusions prepared from oregano, thyme and wild thyme. Food Technology and Biotechnology, 2006, 44(4), 485-492. 
[122] Gulcin, I.; Sat, I. G.; Beydemir, S.; Elmastas, M.; Kufrevioglu, O. I., Comparison of antioxidant activity of clove (Eugenia caryophylata Thunb) buds and lavender (Lavandula stoechas L.). Food Chemistry, 2004, 87(3), 393-400.

[123] Komes, D.; Belscak-Cvitanovic, A.; Horzic, D.; Rusak, G.; Likic, S.; Berendika, M., Phenolic Composition and Antioxidant Properties of Some Traditionally Used Medicinal Plants Affected by the Extraction Time and Hydrolysis. Phytochemical Analysis, 2011, 22(2), 172-180.

[124] Ito, N.; Nihei, T.; Kakuda, R.; Yaoita, Y.; Kikuchi, M., Five new phenylethanoid glycosides from the whole plants of Lamium purpureum L. Chemical \& Pharmaceutical Bulletin, 2006, 54(12), 1705-1708.

[125] Sugaya, K.; Hashimoto, F.; Ono, M.; Ito, Y.; Masuoka, C.; Nohara, T., AntiOxidative Constituents from Leonurii Herba (Leonurus japonicus). Food Science and Technology International Tokyo, 1998, 4(4), 278-281.

[126] Seo, H. K.; Kim, J. S.; Kang, S. S., Labdane Diterpenes and Flavonoids from Leonurus japonicus. Helvetica Chimica Acta, 2010, 93(10), 2045-2051.

[127] Pan, S.-M.; Ding, H.-Y.; Chang, W.-L.; Lin, H.-C., Phenols from the Aerial Parts of Leonurus sibiricus. The Chinese Pharmaceutical Journal, 2006, 58, 35-40.

[128] She, G. M.; Xu, C.; Liu, B.; Shi, R. B., Polyphenolic Acids from Mint (the Aerial of Mentha haplocalyx Briq.) with DPPH Radical Scavenging Activity. Journal of Food Science, 2010, 75(4), C359-C362.

[129] Horwath, A. B.; Grayer, R. J.; Keith-Lucas, D. M.; Simmonds, M. S. J., Chemical characterisation of wild populations of Thymus from different climatic regions in southeast Spain. Biochemical Systematics and Ecology, 2008, 36(2), 117-133.

[130] Marin, P. D.; Grayer, R. J.; Kite, G. C.; Matevski, V., External leaf flavonoids of Thymus species from Macedonia. Biochemical Systematics and Ecology, 2003, 31(11), 1291-1307.

[131] Corticchiato, M.; Bernardini, A.; Costa, J.; Bayet, C.; Saunois, A.; Voirin, B., Free flavonoid aglycones from Thymus-herba-barona and its monoterpenoid chemotypes. Phytochemistry, 1995, 40(1), 115-120.

[132] Alipieva, K. I.; Taskova, R. M.; Evstatieva, L. N.; Handjieva, N. V.; Popov, S. S., Benzoxazinoids and iridoid glucosides from four Lamium species. Phytochemistry, 2003, 64(8), 1413-1417.

[133] Zhang, X.; Zhang, Z., Heart-cut two-dimensional separation method via hyphenation of micellar electrokinetic capillary chromatography and capillary zone electrophoresis using analyte focusing by micelle collapse. Journal of Chromatography B, 2011, 879(19), 1641-1646.

[134] Areias, F. M.; Valentao, P.; Andrade, P. B.; Moreira, M. M.; Amaral, J.; Seabra, R. M., HPLC/DAD analysis of phenolic compounds from lavender and its application to quality control. Journal of Liquid Chromatography \& Related Technologies, 2000, 23(16), 2563-2572. 
[135] Nagy, T. O.; Solar, S.; Sontag, G.; Koenig, J., Identification of phenolic components in dried spices and influence of irradiation. Food Chemistry, 2011, 128(2), 530-534.

[136] Sosa, S.; Altinier, G.; Politi, M.; Braca, A.; Morelli, I.; Della Loggia, R., Extracts and constituents of Lavandula multifida with topical anti-inflammatory activity. Phytomedicine, 2005, 12(4), 271-277.

[137] Janicsak, G.; Mathe, I.; Miklossy-Vari, V.; Blunden, G., Comparative studies of the rosmarinic and caffeic acid contents of Lamiaceae species. Biochemical Systematics and Ecology, 1999, 27(7), 733-738.

[138] Hanganu, D.; Vlase, L.; Olah, N., LC/MS analysis of isoflavones from Fabaceae species extracts. Farmacia, 2010, 58(2), 177-183.

[139] Cui, S. Y.; Chen, X. G.; Hu, Z., Identification and determination of ecdysone and phenylpropanoid glucoside and flavonoids in Lamium maculatum by carpillary zone electrophoresis. Biomedical Chromatography, 2003, 17(7), 477-482.

[140] Akowuah, G. A.; Zhari, I., Effect of Extraction Temperature on Stability of Major Polyphenols and Antioxidant Activity of Orthosiphon stamineus Leaf. Journal of Herbs, Spices \& Medicinal Plants, 2010, 16(3-4), 160-166.

[141] Ozgen, U.; Mavi, A.; Terzi, Z.; Kazaz, C.; Asci, A.; Kaya, Y.; Secen, H., Relationship Between Chemical Structure and Antioxidant Activity of Luteolin and Its Glycosides Isolated from Thymus sipyleus subsp sipyleus var. sipyleus. Records of Natural Products, 2011, 5(1), 12-21.

[142] Ozgen, U.; Mavi, A.; Terzi, Z.; Yildirim, A.; Coskun, M.; Houghton, P. J., Antioxidant properties of some medicinal Lamiaceae (Labiatae) species. Pharmaceutical Biology, 2006, 44(2), 107-112.

[143] Dai, J.; Mumper, R. J., Plant Phenolics: Extraction, Analysis and Their Antioxidant and Anticancer Properties. Molecules, 2010, 15(10), 7313-7352.

[144] Singleton, V. L.; Rossi, J. A., Colorimetry of total phenolics with phosphomolybdic-phosphotungstic acid reagents. American Journal of Enology and Viticulture, 1965, 16, 144-158.

[145] Appel, H. M.; Govenor, H. L.; D'Ascenzo, M.; Siska, E.; Schultz, J. C., Limitations of Folin assays of foliar phenolics in ecological studies. Journal of Chemical Ecology, 2001, 27(4), 761-778.

[146] Roura, E.; Andres-Lacueva, C.; Estruch, R.; Lamucla-Raventos, R. M., Total polyphenol intake estimated by a modified Folin-Ciocalteu assay of urine. Clinical Chemistry, 2006, 52(4), 749-752.

[147] Kulisic, T.; Dragovic-Uzelac, V.; Milos, M., Antioxidant Activity of Aqueous Tea Infusions Prepared from Oregano, Thyme and Wild Thyme. Food Technology Biotechnology, 2006, 44, 485-492.

[148] Stalikas, C. D., Extraction, separation, and detection methods for phenolic acids and flavonoids. Journal of Separation Science, 2007, 30(18), 3268-3295.

[149] Torras-Claveria, L.; Jauregui, O.; Bastida, J.; Codina, C.; Viladomat, F., Antioxidant activity and phenolic composition of lavandin (Lavandula $\mathrm{x}$ intermedia 
emeric ex loiseleur) waste. Journal of Agricultural and Food Chemistry, 2007, 55(21), 8436-8443.

[150] Alipieva, K.; Kokubun, T.; Taskova, R.; Evstatieva, L.; Handjieva, N., LC-ESI-MS analysis of iridoid glucosides in Lamium species. Biochemical Systematics and Ecology, 2007, 35(1), 17-22.

[151] Santana, C. M.; Ferrera, Z. S.; Padron, M. E. T.; Rodriguez, J. J. S., Methodologies for the Extraction of Phenolic Compounds from Environmental Samples: New Approaches. Molecules, 2009, 14(1), 298-320.

[152] Dorman, H. J. D.; Kosar, M.; Baser, K. H. C.; Hiltunen, R., Phenolic Profile and Antioxidant Evaluation of Mentha x piperita L., (Peppermint) Extracts. Natural Product Communications, 2009, 4(4), 535-542.

[153] Blazquez, M. A.; Manez, S.; Zafrapolo, M. C., Further flavonoids and other phenolics of Thymus-webbianus rouy. Zeitschrift Fur Naturforschung $C$-a Journal of Biosciences, 1994, 49(9-10), 687-688.

[154] Fecka, I.; Turek, S., Determination of polyphenolic compounds in commercial herbal drugs and spices from Lamiaceae: thyme, wild thyme and sweet marjoram by chromatographic techniques. Food Chemistry, 2008, 108(3), 1039-1053.

[155] Lee, I. C.; Bae, J. S.; Kim, T.; Kwon, O. J.; Kim, T. H., Polyphenolic Constituents from the Aerial Parts of Thymus quinquecostatus var. japonica Collected on Ulleung Island. Journal of the Korean Society for Applied Biological Chemistry, 2011, 54(5), 811-816.

[156] Oinonen, P. P.; Jokela, J. K.; Hatakka, A. I.; Vuorela, P. M., Linarin, a selective acetylcholinesterase inhibitor from Mentha arvensis. Fitoterapia, 2006, 77(6), 429-434.

[157] Spiridon, I.; Colceru, S.; Anghel, N.; Teaca, C. A.; Bodirlau, R.; Armatu, A., Antioxidant capacity and total phenolic contents of oregano (Origanum vulgare), lavender (Lavandula angustifolia) and lemon balm (Melissa officinalis) from Romania. Natural Product Research, 2011, 25(17), 1657-1661.

[158] Cuyckens, H.; Claeys, M., Mass spectrometry in the structural analysis of flavonoids. Journal of Mass Spectrometry, 2004, 39(4), 1-15.

[159] Chang, J.-M.; Shen, C.-C.; Huang, Y.-L.; Shieh, B.-J.; Chen, C.-C., Two new glycosides from Leonurus japonicus. Journal of Asian Natural Products Research, 2010, 12(9), 740-744.

[160] Nugroho, A.; Choi, J. K.; Park, J. H.; Lee, K. T.; Cha, B. C.; Park, H. J., Two New Flavonol Glycosides from Lamium amplexicaule L. and Their in vitro Free Radical Scavenging and Tyrosinase Inhibitory Activities. Planta Medica, 2009, 75(4), 364-366.

[161] El-Garf, I.; Grayer, R. e. J.; Kite, G. C.; Veitch, N. C., Hypolaetin 8-O-glucuronide and related flavonoids from Lavandula coronopifolia and L. pubescens. Biochemical Systematics and Ecology, 1999, 27(8), 843-846.

[162] Voirin, B.; Bayet, C.; Faure, O.; Jullien, F., Free flavonoid aglycones as markers of parentage in Mentha aquatica, M. citrata, M. spicata and M. $x$ piperita. Phytochemistry, 1999, 50(7), 1189-1193. 
[163] Maul, R.; Schebb, N.; Kulling, S., Application of LC and GC hyphenated with mass spectrometry as tool for characterization of unknown derivatives of isoflavonoids. Analytical and Bioanalytical Chemistry, 2008, 391(1), 239-250.

[164] Grayer, R. J.; Eckert, M. R.; Veitch, N. C.; Kite, G. C.; Marin, P. D.; Kokubun, T.; Simmonds, M. S. J.; Paton, A. J., The chemotaxonomic significance of two bioactive caffeic acid esters, nepetoidins A and B, in the Lamiaceae. Phytochemistry, 2003, 64(2), 519-528.

[165] Kurkin, V. A.; Lamrini, M.; Klochkov, S. G., Lavandoside from Lavandula spica flowers. Chemistry of Natural Compounds, 2008, 44(2), 169-170.

[166] Kosar, M.; Dorman, H. J. D.; Baser, K. H. C.; Hiltunen, R., Screening of free radical scavenging compounds in water extracts of Mentha samples using a postcolumn derivatization method. Journal of Agricultural and Food Chemistry, 2004, 52(16), 5004-5010.

[167] Shekarchi, M.; Hajimehdipoor, H.; Saeidnia, S.; Gohari, A. R.; Hamedani, M. P., Comparative study of rosmarinic acid content in some plants of Labiatae family. Pharmacognosy Magazine, 2012, 8(29), 37-41.

[168] Costa, P.; Goncalves, S.; Andrade, P. B.; Valentao, P.; Romano, A., Inhibitory effect of Lavandula viridis on $\mathrm{Fe}(2+)$-induced lipid peroxidation, antioxidant and anticholinesterase properties. Food Chemistry, 2011, 126(4), 1779-1786.

[169] Blazekovic, B.; Vladimir-Knezevic, S.; Brantner, A.; Stefan, M. B., Evaluation of Antioxidant Potential of Lavandula $x$ intermedia Emeric ex Loisel. 'Budrovka': A Comparative Study with L. angustifolia Mill. Molecules, 2010, 15(9), 5971-5987.

[170] Kovatcheva-Apostolova, E. G.; Georgiev, M. I.; Ilieva, M. P.; Skibsted, L. H.; Rodtjer, A.; Andersen, M. L., Extracts of plant cell cultures of Lavandula vera and Rosa damascena as sources of phenolic antioxidants for use in foods. European Food Research and Technology, 2008, 227(4), 1243-1249.

[171] Budzianowski, J.; Skrzypczak, L., Phenylpropanoid esters from Lamium album flowers. Phytochemistry, 1995, 38(4), 997-1001.

[172] Jimenez, C.; Riguera, R., Phenylethanoid glycosides in plants: Structure and biological activity. Natural Product Reports, 1994, 11(6), 591-606.

[173] Deng, Y.; He, L.; Li, W.; Wang, H., [Studies on chemical constituents in herb of Lamium maculatum L. var Kansuense]. Zhongguo Zhong yao za zhi = Zhongguo zhongyao zazhi = China journal of Chinese materia medica, 2003, 28(8), 730-2.

[174] Calis, I.; Ersoz, T.; Tasdemir, D.; Ruedi, P., Two phenylpropanoid glycosides from Leonurus-glaucescens. Phytochemistry, 1992, 31(1), 357-359.

[175] Kuchta, K.; Ortwein, J.; Savtschenko, A.; Briel, D.; Volk, R. B.; Rauwald, H. W., Leonurus cardiaca, L. Japonicus, Leonotis leonurus: Quantitative HPLC and instrumental HPTLC determination of fourteen phenolics. Planta Medica, 2012, 78(11), PJ50.

[176] Milkowska-Leyck, K.; Filipek, B.; Strzelecka, H., Pharmacological effects of lavandulifolioside from Leonurus cardiaca. Journal of Ethnopharmacology, 2002, 80(1), 85-90. 
[177] Gabrieli, C.; Kokkalou, E., A new acetylated glucoside of luteolin and two flavone glucosides from Lavandula stoechas ssp stoechas. Pharmazie, 2003, 58(6), 426-427.

[178] Ferreres, F.; Barberan, F. A. T.; Tomas, F., Flavonoids from Lavandula dentata. Fitoterapia, 1986, 57, 199-200.

[179] Imelouane, B.; Elbachiri, A.; Ankit, M.; Benzeid, H.; Khedid, K., Physico-Chemical Compositions and Antimicrobial Activity of Essential Oil of Eastern Moroccan Lavandula dentata. International Journal of Agriculture and Biology, 2009, 11(2), 113118.

[180] Zaidi, F.; Voirin, B.; Jay, M.; Viricel, M. R., Free flavonoid aglycones from leaves of Mentha pulegium and Mentha suaveolens (Labiatae). Phytochemistry, 1998, 48(6), 991-994.

[181] Ghoulami, S.; Idrissi, A.; Fkih-Tetouani, S., Phytochemical study of Mentha longifolia of Morocco. Fitoterapia, 2001, 72(5), 596-598.

[182] De Almeida, L. F. R.; Delachiave, M. E.; Sannomiya, M.; Vilegas, W.; Dos Santos, L. C.; Mancini, E.; De Feo, V., In vitro allelopathic potential of Leonurus sibiricus L. leaves. Journal of Plant Interactions, 2008, 3(1), 39-48.

[183] Boalino, D. M.; McLean, S.; Reynolds, W. F.; Tinto, W. F., Labdane diterpenes of Leonurus sibiricus. Journal of Natural Products, 2004, 67(4), 714-717.

[184] Giang, P. M.; Son, P. T.; Matsunami, K.; Otsuka, H., New labdane-type diterpenoids from Leonurus heterophyllus Sw. Chemical \& Pharmaceutical Bulletin, 2005, 53(8), 938-941.

[185] Tasdemir, D.; Calis, I.; Sticher, O., Labdane diterpenes from Leonurus persicus. Phytochemistry, 1998, 49(1), 137-143.

[186] Masteikova, R.; Muselik, J.; Bernatoniene, J.; Majiene, D.; Savickas, A.; Malinauskas, F.; Bernatoniene, R.; Peciura, R.; Chalupova, Z.; Dvorackova, K., Antioxidant activity of tinctures prepared from hawthorn fruits and motherwort herb. Ceska a Slovenska farmacie : casopis Ceske farmaceuticke spolecnosti a Slovenske farmaceuticke spolecnosti, 2008, 57(1), 35-8.

[187] Miura, K.; Nakatani, N., Antioxidative activity of flavonoids from Thyme (Thymusvulgaris L). Agricultural and Biological Chemistry, 1989, 53(11), 3043-3045.

[188] Marin, P. D.; Grayer, R. J.; Kite, G. C.; Veljic, M., External flavones from Thymus striatus Vahl (Lamiaceae). Biochemical Systematics and Ecology, 2005, 33(11), 11791182.

[189] Masteikova, R.; Muselik, J.; Bernatoniene, J.; Majiene, D.; Savickas, A.; Malinauskas, F.; Bernatoniene, R.; Peciura, R.; Chalupova, Z.; Dvorackova, K., [Antioxidant activity of tinctures prepared from hawthorn fruits and motherwort herb]. Ceska a Slovenska farmacie : casopis Ceske farmaceuticke spolecnosti a Slovenske farmaceuticke spolecnosti, 2008, 57(1), 35-8.

[190] Cong, Y.; Wang, J. H.; Li, X., A new flavonoside from Leonurus heterophyllus. Journal of Asian Natural Products Research, 2005, 7(3), 273-277. 
[191] Jaeger, A. K.; Almqvist, J. P.; Vangsoe, S. A. K.; Stafford, G. I.; Adsersen, A.; Van Staden, J., Compounds from Mentha aquatica with affinity to the GABAbenzodiazepine receptor. South African Journal of Botany, 2007, 73(4), 518-521.

[192] Wang, M.; Li, J.; Ho, G. S.; Peng, X.; Ho, C. T., Isolation and identification of antioxidative flavonoid glycosides from thyme (Thymus vulgaris L.). Journal of Food Lipids, 1998, 5(4), 313-321.

[193] Sanchez-Valle, V.; Chavez-Tapia, N. C.; Uribe, M.; Mendez-Sanchez, N., Role of Oxidative Stress and Molecular Changes in Liver Fibrosis: A Review. Current Medicinal Chemistry, 2012, 18, 18.

[194] Zhu, H.; Jia, Z.; Misra, H.; Li, Y. R., Oxidative stress and redox signaling mechanisms of alcoholic liver disease: Updated experimental and clinical evidence. Journal of Digestive Diseases, 2012, 13(3), 133-142.

[195] Angelopoulou, R.; Lavranos, G.; Manolakou, P., ROS in the aging male: Model diseases with ROS-related pathophysiology. Reproductive Toxicology, 2009, 28(2), 167-171.

[196] Hitchon, C. A.; El-Gabalawy, H. S., Oxidation in rheumatoid arthritis. Arthritis Research \& Therapy, 2004, 6(6), 265-278.

[197] Turrens, J. F., Mitochondrial formation of reactive oxygen species. The Journal of Physiology, 2003, 552(2), 335-344.

[198] Krishnaiah, D.; Sarbatly, R.; Nithyanandam, R., A review of the antioxidant potential of medicinal plant species. Food and Bioproducts Processing, 2011, 89(3), 217-233.

[199] Tsakona, S.; Galanakis, C. M.; Gekas, V., Hydro-Ethanolic Mixtures for the Recovery of Phenols from Mediterranean Plant Materials. Food and Bioprocess Technology, 2012, 5(4), 1384-1393.

[200] MacDonald-Wicks, L. K.; Wood, L. G.; Garg, M. L., Methodology for the determination of biological antioxidant capacity in vitro: a review. Journal of the Science of Food and Agriculture, 2006, 86(13), 2046-2056.

[201] Pisoschi, A. M.; Negulescu, G. P., Methods for Total Antioxidant Activity Determination: A Review. Biochemistry and Analytical Biochemistry, 2011, 1(1), 1-10.

[202] Saikat, S.; Raja, C., The role of antioxidants in human health. In Oxidative Stress: Diagnostics, Prevention, and Therapy, American Chemical Society: 2011; Vol. 1083, pp 1-37.

[203] Niki, E., Assessment of Antioxidant Capacity in vitro and in vivo. Free Radical Biology and Medicine, 2010, 49(4), 503-515.

[204] Lopez, V.; Akerreta, S.; Casanova, E.; Garcia-Mina, J. M.; Cavero, R. Y.; Calvo, M. I., In vitro antioxidant and anti-rhizopus activities of lamiaceae herbal extracts. Plant Foods for Human Nutrition, 2007, 62(4), 151-155.

[205] Messaoud, C.; Chograni, H.; Boussaid, M., Chemical composition and antioxidant activities of essential oils and methanol extracts of three wild Lavandula L. species. Natural Product Research, 2011, 1-9. 
[206] Sariri, R.; Seifzadeh, S.; Sajedi, R. H., Anti-tyrosinase and antioxidant activity of Lavandula sp. extracts. Pharmacologyonline, 2009, 2, 413-420.

[207] Robu, S.; Aprotosoaie, A. C.; Miron, A.; Cioanca, O.; Stanescu, U.; Hancianu, M., In vitro antioxidant activity of ethanolic extracts from some Lavandula species cultivated in Romania. Farmacia, 2012, 60(3), 394-401.

[208] Kovacheva, E.; Georgiev, M.; Pashova, S.; Angelova, M.; Ilieva, M., Radical quenching by rosmariuic acid from Lavandula vera MM cell culture. Zeitschrift Fur Naturforschung C-a Journal of Biosciences, 2006, 61(7-8), 517-520.

[209] Tsai, P. J.; Tsai, T. H.; Yu, C. H.; Ho, S. C., Comparison of NO-scavenging and NO-suppressing activities of different herbal teas with those of green tea. Food Chemistry, 2007, 103(1), 181-187.

[210] Armatu, A.; Colceru-Mihul, S.; Bubueanu, C.; Draghici, E.; Pirvu, L., Evaluation of antioxidant and free scavenging potential of some Lamiaceae species growing in Romania. Romanian Biotechnological Letters, 2010, 15(3), 5274-5280.

[211] Trouillas, P.; Calliste, C.-A.; Allais, D.-P.; Simon, A.; Marfak, A.; Delage, C.; Duroux, J.-L., Antioxidant, anti-inflammatory and antiproliferative properties of sixteen water plant extracts used in the Limousin countryside as herbal teas. Food Chemistry, 2003, 80(3), 399-407.

[212] Valyova, M. S.; Dimitrova, M. A.; Ganeva, Y. A.; Mihova Kapchina-Toteva, V.; Petkova Yordanova, Z., Evaluation of antioxidant and free radical scavenging potential of Lamium album L. growing in Bulgaria. Journal of Pharmacy Research, 2011, 4(4), 945-947.

[213] Yalcin, F. N.; Kaya, D.; Kilic, E.; Ozalp, M.; Ersoz, T.; Calis, İ., Antimicrobial and Free Radical Scavenging Activities of Some Lamium Species from Turkey. Hacettepe University Journal of the Faculty of Pharmacy, 2007, 27(11-22).

[214] Prieto, P.; Pineda, M.; Aguilar, M., Spectrophotometric Quantitation of Antioxidant Capacity through the Formation of a Phosphomolybdenum Complex: Specific Application to the Determination of Vitamin E. Analytical Biochemistry, 1999, 269(2), 337-341.

[215] Arumugam, P.; Ramamurthy, P.; Santhiya, S. T.; Ramesh, A., Antioxidant activity measured in different solvent fractions obtained from Mentha spicata Linn.: An analysis by ABTS(.+) decolorization assay. Asia Pacific Journal of Clinical Nutrition, 2006, 15(1), 119-124.

[216] Lopez, V.; Martin, S.; Pilar Gomez-Serranillos, M.; Emilia Carretero, M.; Jager, A. K.; Isabel Calvo, M., Neuroprotective and Neurochemical Properties of Mint Extracts. Phytotherapy Research, 2010, 24(6), 869-874.

[217] Jain, S.; Jain, D. K.; Balekar, N., In vivo Antioxidant activity of ethanolic extract of Mentha pulegium leaf against $\mathrm{CCl} 4$ induced toxicity in rats. Asian Pacific Journal of Tropical Biomedicine, 2012, 2(2, Supplement), S737-S740.

[218] Amarowicz, R.; Zegarska, Z.; Rafalowski, R.; Pegg, R. B.; Karamac, M.; Kosinska, A., Antioxidant activity and free radical-scavenging capacity of ethanolic extracts of thyme, oregano, and marjoram. European Journal of Lipid Science and Technology, 2009, 111(11), 1111-1117.

Pereira O. R., 2013 | CHARACTERIZATION OF PHENOLIC CONSTITUENTS OF MEDICINAL PLANTS AND EVALUATION OF PHARMACOLOGICAL ACTIVITIES: FOCUS IN ANTIOXIDANT AND 
[219] Bueyuekbalci, A.; El, S. N., Determination of In vitro antidiabetic effects, antioxidant activities and phenol contents of some herbal teas. Plant Foods for Human Nutrition, 2008, 63(1), 27-33.

[220] Albano, S. M.; Miguel, M. G., Biological activities of extracts of plants grown in Portugal. Industrial Crops and Products, 2011, 33(2), 338-343.

[221] Ulukanli, Z.; Cigremis, Y.; Ilcim, A., In vitro antimicrobial and antioxidant activity of acetone and methanol extracts from Thymus leucotrichius (Lamiaceae). European Review for Medical and Pharmacological Sciences, 2011, 15(6), 649-657.

[222] Lee, M. J.; Lee, H. S.; Park, S. D.; Moon, H. I.; Park, W. H., Leonurus sibiricus Herb Extract Suppresses Oxidative Stress and Ameliorates Hypercholesterolemia in C57BL/6 Mice and TNF-alpha Induced Expression of Adhesion Molecules and LectinLike Oxidized LDL Receptor-1 in Human Umbilical Vein Endothelial Cells. Bioscience Biotechnology and Biochemistry, 2010, 74(2), 279-284.

[223] Luis, A.; Domingues, F.; Gil, C.; Duarte, A. P., Antioxidant activity of extracts of Portuguese shrubs: Pterospartum tridentatum, Cytisus scoparius and Erica spp. Journal of Medicinal Plants Research, 2009, 3(11), 886-893.

[224] Raja, S.; Ahamed, H. N.; Kumar, V.; Mukherjee, K.; Bandyopadhyay, A.; Mukherjee, P. K., Exploring the Effect of Cytisus Scoparius on Markers of Oxidative Stress in Rats. Iranian Journal of Pharmacology \& Therapeutics, 2007, 6(1), 15-21.

[225] Costa, G.; Francisco, V.; Lopes, M. C.; Cruz, M. T.; Batista, M. T., Intracellular Signaling Pathways Modulated by Phenolic Compounds: Application for New AntiInflammatory Drugs Discovery. Current Medicinal Chemistry, 2012, 19(18), 2876-2900.

[226] Zhang, J.-M.; An, J., Cytokines, inflammation, and pain. International Anesthesiology Clinics, 2007, 45(2), 27-37.

[227] Gautam, R.; Jachak, S. M., Recent developments in anti-inflammatory natural products. Medicinal Research Reviews, 2009, 29(5), 767-820.

[228] Schottenfeld, D.; Beebe-Dimmer, J., Chronic inflammation: A common and important factor in the pathogenesis of neoplasia. CA-A Cancer Journal for Clinicians, 2006, 56(2), 69-83.

[229] Hwang, S. H.; Wecksler, A. T.; Wagner, K.; Hammock, B. D., Rationally Designed Multitarget Agents against Inflammation and Pain. Current Medicinal Chemistry, 2013, 20(13), 1783-99.

[230] Gosslau, A.; Li, S.; Ho, C.-T.; Chen, K. Y.; Rawson, N. E., The importance of natural product characterization in studies of their anti-inflammatory activity. Molecular Nutrition \& Food Research, 2011, 55(1), 74-82.

[231] Falcao, H. d. S.; Lima, I. O.; Santos, V. L. d.; Dantas, H. d. F.; Diniz, M. d. F. F. M.; Barbosa-Filho, J. M.; Batista, L. M., Review of the plants with anti-inflammatory activity studied in Brazil. Revista Brasileira de Farmacognosia, 2005, 15, 381-391.

[232] Dey, M.; Ribnicky, D.; Kurmukov, A. G.; Raskin, I., In Vitro and in Vivo AntiInflammatory Activity of a Seed Preparation Containing Phenethylisothiocyanate. Journal of Pharmacology and Experimental Therapeutics, 2006, 317(1), 326-333. 
[233] Eddouks, M.; Chattopadhyay, D.; Zeggwagh, N. A., Animal Models as Tools to Investigate Antidiabetic and Anti-Inflammatory Plants. Evidence-Based Complementary and Alternative Medicine, 2012.

[234] Souto, A. L.; Tavares, J. F.; da Silva, M. S.; Diniz, M.; de Athayde, P. F.; Barbosa, J. M., Anti-Inflammatory Activity of Alkaloids: An Update from 2000 to 2010. Molecules, 2011, 16(10), 8515-8534.

[235] Prieto, J. M.; Bader, A.; Martini, F.; Rios, J. L.; Morelli, I., Screening of some rare endemic Italian plants for inhibitory activity on 5-lipoxygenase. Fitoterapia, 2005, 76(78), 725-727.

[236] Islam, M. A.; Ahmed, F.; Das, A. K.; Bachar, S. C., Analgesic and antiinflammatory activity of Leonurus sibiricus. Fitoterapia, 2005, 76(3-4), 359-362.

[237] Hajhashemi, V.; Ghannadi, A.; Sharif, B., Anti-inflammatory and analgesic properties of the leaf extracts and essential oil of Lavandula angustifolia Mill. Journal of Ethnopharmacology, 2003, 89(1), 67-71.

[238] Dulger, B.; Hacioglu, N., Antibacterial activity of endemic Lamium tenuiflorum. Asian Journal of Chemistry, 2008, 20(8), 6577-6581.

[239] Warda, K.; Markouk, M.; Bekkouche, K.; Larhsini, M.; Abbad, A.; Romane, A.; Bouskraoui, M., Antibacterial evaluation of selected Moroccan medicinal plants against Streptococcus pneumoniae. African Journal of Pharmacy and Pharmacology, 2009, 3(3), 101-104.

[240] Stefanovic, O.; Comic, L., Inhibitory effect of Cytisus nigricans L. and Cytisus capitatus Scop. on growth of bacteria. African Journal of Microbiology Research, 2011, 5(27), 4725-4730.

[241] Moskova-Doumanova, V.; Miteva, G.; Dimitrova, M.; Topouzova-Hristova, T.; Kapchina, V., Methanol and chloroform extracts from Lamium album L. affect cell properties of A549 cancer lung cell line. Medical Biotechnology, 2011, 26, 120-125.

[242] Yi, W. G.; Wetzstein, H. Y., Anti-tumorigenic activity of five culinary and medicinal herbs grown under greenhouse conditions and their combination effects. Journal of the Science of Food and Agriculture, 2011, 91(10), 1849-1854.

[243] Arumugam, P.; Ramamurthy, P.; Ramesh, A., Antioxidant and Cytotoxic Activities of Lipophilic and Hydrophilic Fractions of Mentha Spicata L. (Lamiaceae). International Journal of Food Properties, 2010, 13(1), 23-31.

[244] Caillet, S.; Lessard, S.; Lamoureux, G.; Lacroix, M., Umu test applied for screening natural antimutagenic agents. Food Chemistry, 2011, 124(4), 1699-1707.

[245] Sun, Z.-X.; Zhang, Y.-H.; Cheng, S.; Ma, Q.-W.; Guo, S.-L.; Zhang, J.-B., [Antitumor effect of ethanol extracts from Thymus quinquecostatus Celak on human leukemia cell line]. Journal of Chinese Integrative Medicine, 2005, 3(5), 382-5.

[246] Elhabazi, K.; Ouacherif, A.; Laroubi, A.; Aboufatima, R.; Abbad, A.; Benharref, A.; Zyad, A.; Chait, A.; Dalal, A., Analgesic activity of three thyme species, Thymus satureioides, Thymus maroccanus and Thymus leptobotrys. African Journal of Microbiology Research, 2008, 2(10), 262-267. 
[247] Ellman, G. L.; Courtney, K. D.; Andres, V.; Featherstone, R. M., A new and rapid colorimetric determination of acetylcholinesterase activity. Biochemical Pharmacology, 1961, 7(2), 88-90.

[248] Buyukokuroglu, M. E.; Gepdiremen, A.; Hacimuftuoglu, A.; Oktay, M., The effects of aqueous extract of Lavandula angustifolia flowers in glutamate-induced neurotoxicity of cerebellar granular cell culture of rat pups. Journal of Ethnopharmacology, 2003, 84(1), 91-94.

[249] Kashani, M. S.; Tavirani, M. R.; Talaei, S. A.; Salami, M., Aqueous extract of lavender (Lavandula angustifolia) improves the spatial performance of a rat model of Alzheimer's disease. Neuroscience Bulletin, 2011, 27(2), 99-106.

[250] Gilani, A. H.; Aziz, N.; Khan, M. A.; Shaheen, F.; Jabeen, Q.; Siddiqui, B. S.; Herzig, J. W., Ethnopharmacological evaluation of the anticonvulsant, sedative and antispasmodic activities of Lavandula stoechas L. Journal of Ethnopharmacology, 2000, 71(1-2), 161-167.

[251] Grande, S.; Bogani, P.; De Saizieu, A.; Schueler, G.; Galli, C.; Visioli, F., Vasomodulating potential of Mediterranean wild plant extracts. Journal of Agricultural and Food Chemistry, 2004, 52(16), 5021-5026.

[252] Loziene, K.; Venskutonis, P. R.; Sipailiene, A.; Labokas, J., Radical scavenging and antibacterial properties of the extracts from different Thymus pulegioides $\mathrm{L}$. chemotypes. Food Chemistry, 2007, 103(2), 546-559.

[253] Bouayed, J.; Piri, K.; Rammal, H.; Dicko, A.; Desor, F.; Younos, C.; Soulimani, R., Comparative evaluation of the antioxidant potential of some Iranian medicinal plants. Food Chemistry, 2007, 104(1), 364-368.

[254] Dulger, B., Antifungal activity of Lamium tenuiflorum against some medical yeast Candida and Cryptococcus species. Pharmaceutical Biology, 2009, 47(5), 467-470.

[255] Camejo-Rodrigues, J.; Ascensao, L.; Bonet, M. A.; Valles, J., An ethnobotanical study of medicinal and aromatic plants in the Natural Park of "Serra de Sao Mamede" (Portugal). Journal of Ethnopharmacology, 2003, 89(2-3), 199-209.

[256] Gonzalez-Tejero, M. R.; Casares-Porcel, M.; Sanchez-Rojas, C. P.; RamiroGutierrez, J. M.; Molero-Mesa, J.; Pieroni, A.; Giusti, M. E.; Censorii, E.; de Pasquale, C.; Della, A.; Paraskeva-Hadijchambi, D.; Hadjichambis, A.; Houmani, Z.; ElDemerdash, M.; El-Zayat, M.; Hmamouchi, M.; ElJohrig, S., Medicinal plants in the Mediterranean area: Synthesis of the results of the project Rubia. Journal of Ethnopharmacology, 2008, 116(2), 341-357. 
6. RESUMEN 



\section{UNIVERSIDAD DE SALAMANCA \\ FACULTAD DE FARMACIA}

\section{DEPARTAMENTO DE FISIOLOGÍA Y FARMACOLOGÍA}

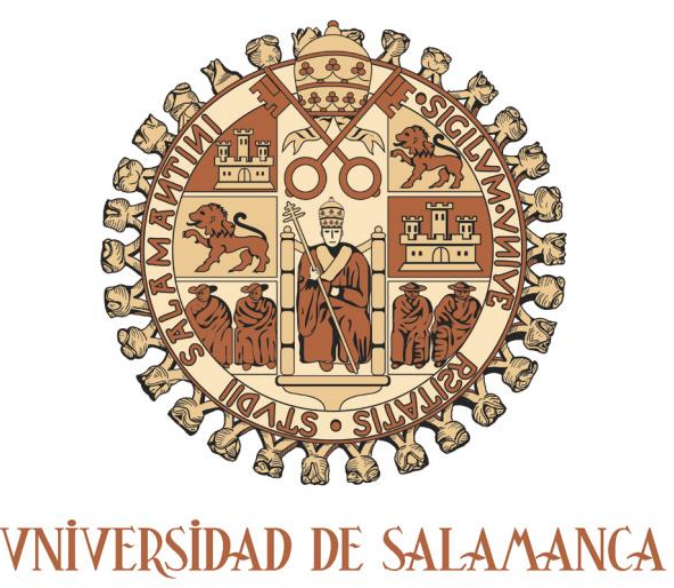

CARACTERIZACION DE COMPUESTOS FENÓLICOS EN

PLANTAS MEDICINALES Y EVALUACIÓN DE ACTIVIDADES FARMACOLÓGICAS: PROPIEDADES

ANTIOXIDANTES Y ANTI-INFLAMATORIAS

RESUMEN DE TESIS DOCTORAL

OLIVIA RODRIGUES PEREIRA 


\subsection{OBJETIVOS}

A pesar del creciente número de estudios centrados en los metabolitos secundarios de las plantas, la caracterización del perfil fenólico de un gran número de especies permanece poco estudiada. Por otra parte, varios estudios indican la relación entre los compuestos fenólicos presentes en las plantas y sus efectos beneficiosos para la salud humana, sin embargo, el mecanismo exacto de acción sigue siendo poco claro y las propiedades farmacológicas que se les asignan están, en su mayoría, basadas en el conocimiento popular.

Los perfiles en compuestos fenólicos de las especie vegetales Cytisus multiflorus, Lamium album L., Lavandula dentata L., Leonurus cardiaca, Mentha aquatica L. y Thymus $x$ citriodorus están muy poco estudiados y en algunos casos aún se desconocen, por lo que son necesarios más estudios. De igual modo, varias propiedades han sido asignadas a distintos extractos de plantas, sin embargo, se necesitan más investigaciones científicas para demostrar las propiedades beneficiosas, ya que, en la mayoría de los casos, los efectos biológicos se han probado exclusivamente en modelos in vitro. En este sentido, el principal objetivo de esta Tesis Doctoral fue mejorar el conocimiento de la composición fenólica y también de los efectos beneficiosos de las seis plantas medicinales Cytisus multiflorus, Lamium album L., Lavandula dentata L., Leonurus cardiaca L., Mentha aquatica L. y Thymus $x$ citriodorus. Para ello, se definieron cinco objetivos específicos:

- Caracterizar y cuantificar los componentes fenólicos de extractos etanólicos de Cytisus multiflorus, Lamium album L., Lavandula dentata L., Leonurus cardiaca L., Mentha aquatica L. y Thymus $x$ citriodorus por cromatografía líquida de alta eficiencia asociada con la detección por haz de diodos (HPLC-DAD), espectrometría de masas por electrospray (ESI-MS y $\mathrm{MS}^{n}$ ) y resonancia magnética nuclear (NMR);

- Determinar los efectos antioxidantes de Cytisus multiflorus, Lamium album L., Lavandula dentata L., Leonurus cardiaca L., Mentha aquatica L. y Thymus $x$ citriodorus in vitro y en modelos celulares;

- Evaluar los efectos hepatoprotectores de Cytisus multiflorus, Lamium album L., Lavandula dentata L., Leonurus cardiaca L., Mentha aquatica L. y Thymus $x$ citriodorus en células HepG2 derivadas de hepatoblastoma humano; 
- Evaluar las propiedades anti-inflamatorias del extracto etanólico purificado de Cytisus multiflorus;

- Evaluar el efecto de los extractos etanólicos purificados de Mentha aquatica y Leonurus cardiaca L. mediante experimentos de bioenergética en mitocondrias de hígado de rata. 


\subsection{INTRODUCCIÓN}

Las plantas se utilizan por el hombre desde la antigüedad por sus efectos beneficiosos para la salud aunque en la mayoría de los casos nunca se describieron ni la composición ni el mecanismo químico asociado a cada efecto. En los últimos años, distintas actividades de las plantas se han atribuido a su composición en polifenoles. Dado el amplio espectro de efectos biológicos que se atribuyen a este tipo de compuestos, numerosos estudios se han desarrollado con vistas a su aplicación en la industria farmacéutica, alimentaria y cosmética.

La región mediterránea es abundante en plantas medicinales, tanto en las formas silvestres como cultivadas. De entre las plantas mediterráneas más utilizadas se destacan las especies de la familia Fabaceae y Lamiaceae. La introducción de esta Tesis Doctoral se centró en la descripción general de los géneros Cytisus, Lamium, Lavandula, Leonurus, Mentha y Thymus seguido de un resumen de los métodos de extracción y caracterización de compuestos fenólicos, así como de los principales compuestos fenólicos descritos en extractos de plantas pertenecientes a estos géneros. Por otra parte, se resumen los efectos biológicos descritos en la literatura hasta el momento, para las plantas de los géneros Cytisus, Lamium, Lavandula, Leonurus, Mentha y Thymus. 


\subsection{MÉTODOS}

Los extractos fenólicos de las especies vegetales Cytisus multiflorus, Lamium album L., Lavandula dentata L., Leonurus cardiaca L., Mentha aquatica L. y Thymus $x$ citriodorus se han obtenido por agitación con una solución de $n$-hexano y posterior extracción con una solución acuosa etanólica $80 \%(\mathrm{v} / \mathrm{v})$. La mayor parte de los extractos etanólicos así obtenidos, se sometió a posterior purificación en cartuchos de SPE Strata C18-E, para concentrar los extractos en compuestos fenólicos.

Los extractos resultantes fueron caracterizados y cuantificados por cromatografía líquida de alta eficiencia (HPLC), asociada a espectrometría de masas por electrospray (ESI-MS) en el modo de ionización negativo y $\mathrm{MS}^{n}$. Se realizaron determinaciones de resonancia magnética nuclear (NMR) con el objetivo de asignar la estructura exacta de los principales compuestos fenólicos de algunos de los extractos. Por otra parte, el método de HPLC fue validado en cuanto a su linealidad, precisión instrumental y precisión del método y se hico un estudio de recuperación absoluta para cuantificación de los compuestos fenólicos en el extracto de T. $x$ citriodorus.

Además de la caracterización química, se determinaron varios efectos para las distintas especies vegetales. La actividad antioxidante se determinó en primer lugar in vitro mediante ensayos químicos, utilizando el test de 2,2-difenil-2-picrilhidrazil $\left(\mathrm{DPPH}^{\circ}\right)$ y el del cálculo del poder reductor. La toxicidad de los extractos se evaluó en células HepG2 de hepatoblastoma humano, mediante la prueba del MTT. Además, se midió el efecto protector de cada extracto $(50 \mu \mathrm{g} / \mathrm{ml})$ frente al aumento de producción de las especies reactivas de oxígeno (ROS) en un modelo de estrés químico inducido en las células HepG2 por incubación con dicromato potásico (DK), después de $48 \mathrm{~h}$ de incubación, por citometría de flujo. Por otro lado, el efecto citoprotector de los extractos de las plantas se evaluó por estudios de viabilidad en el mismo modelo celular, exponiendo las células a los extractos y a DK por periodos de 6 y 72 horas de incubación. Estos ensayos celulares se realizaron también en compuestos fenólicos individuales puros, obtenidos comercialmente (apigenina, crisina, eriodictiol, quercetina, luteolina, naringenina, ácido rosmarínico y verbascósido) y con mezclas que simulan la composición fenólica de los extractos a $50 \mu \mathrm{g} / \mathrm{mL}$, segun las cantidades previamente determinadas de cada compuesto en las especies vegetales, para mimetizar los efectos de los extractos que demostraron más actividad.

En esta Tesis Doctoral también se evaluó el efecto anti-inflamatorio del extracto de $C$. multiflorus utilizando como modelo de inflamación la línea celular de macrófagos RAW 
264.7. Se empezó por estudiar el efecto de distintas concentraciones del extracto $C$. multiflorus sobre la viabilidad de los macrófagos RAW 264.7 mediante el ensayo del MTT. Las propiedades anti-inflamatorias se evaluaron in vitro mediante la determinación de las cantidades de óxido nítrico ( $\mathrm{NO}^{\bullet}$ ) en un modelo químico y en macrófagos RAW 264.7 estimulados con lipopolisacárido. Además, utilizando western blot se determinaron, en el mismo modelo celular, los efectos del extracto de $C$. multiflorus sobre la expresión de dos enzimas clave en los procesos inflamatorios, la ciclooxigenasa-2 (COX-2) y la óxido nítrico sintasa inducible (iNOS). También se evaluó mediante un modelo químico el efecto inhibidor del extracto frente a la actividad de la 5-lipoxigenasa (5-LOX).

Igualmente, se llevaron a cabo ensayos de bioenergética mitocondrial para extractos de las especies vegetales $L$. cardiaca y $M$. aquatica. Para ello, se evaluó la influencia de dos concentraciones distintas de extractos de las plantas en parámetros respiratorios como el estado 2 , estado 3 y estado 4 , la razón de control respiratorio (RCR) y la razón $\mathrm{P} / \mathrm{O}$, así como el potencial de membrana. 


\subsection{RESULTADOS Y DISCUSIÓN}

\subsubsection{Caracterización y cuantificación de compuestos fenólicos presentes en Thymus $x$ citriodorus usando un método validado de HPLC-UV y ESI-MS}

Thymus $x$ citriodorus, también conocido como tomillo de limón, es una planta utilizada para fines medicinales y culinarios. A pesar de su amplio uso en forma de infusión o como condimento en varios platos, la composición fenólica de esta especie vegetal es completamente desconocida. Así, esta parte del trabajo tuvo como objetivo validar un método de HPLC que fue utilizado en la determinación e cuantificación de los compuestos fenólicos presentes en al extracto de T. $x$ citriodorus.

El fraccionamiento del extracto etanólico mediante HPLC-DAD y la posterior análisis de las fracciones recogidas por ESI-MS ${ }^{n}$, permitieron identificar trece compuestos fenólicos, para algunos de ellos se pudo confirmar su identidad por RMN. En conjunto, las técnicas permitieron determinar que el extracto de T. $x$ citriodorus contiene ácido rosmarínico y otros ácidos fenólicos menos comunes, así como derivados de flavonoides, que incluen la flavonas luteolina, apigenina y crisoeriol, las flavanonas naringenina y eriodictiol, y el flavonol quercetagetin. Los datos de HPLC y ESI-MS ${ }^{n}$ están representados en la Tabla 1 y los de NMR en la Tabla 2.

En lo que respecta a los ácidos fenólicos, el ácido rosmarínico (fracción 9), fue identificado por su tiempo de retención, espectro UV-Vis y por los datos obtenidos en los experimentos de ESI-MS ${ }^{n}$ (Tabla 1) y NMR (Tabla 2). Además de este ácido fenólico el extracto de T. $x$ citriodorus contiene un derivado cafeico del ácido rosmarínico que probablemente corresponde al compuesto ácido 3'-O-(8"-Zcaffeoyl)rosmarínico que ha sido previamente detectado en extractos de T. vulgaris. 
Tabla 1 - Datos de HPLC/DAD y ESI/MS ${ }^{n}$ de las fracciones de T. $x$ citriodorus analizadas.

\begin{tabular}{|c|c|c|c|c|c|}
\hline Fracción & $\begin{array}{c}\mathbf{R T} \\
(\min )\end{array}$ & $\begin{array}{l}\lambda_{\max } \\
(\mathrm{nm})\end{array}$ & {$[\mathrm{M}-\mathrm{H}]^{-}$} & $\begin{array}{c}\text { ESI }^{-} \mathbf{M S}^{\mathbf{n}} \\
\text { (Abundance) }\end{array}$ & Compuesto \\
\hline \multirow[t]{2}{*}{1} & 4.3 & 283,327 & 611 & $\begin{array}{l}\mathrm{MS}^{2}[611]: \text { 449(100), 287(15); } \\
\mathrm{MS}^{3}[449]: \text { 287(100), 151(<1); } \\
\mathrm{MS}^{4}[287]: \text { 269(2), 151(100); } \\
\mathrm{MS}^{5}[151]: 107\end{array}$ & Eriodictiol-di-O-hexósido \\
\hline & & & 387 & $\begin{array}{l}\mathrm{MS}^{2}[387]: \text { 369(15), 225(5), 207(100), } \\
\text { 163(10), 119(1); } \mathrm{MS}^{3}[207]: 163 \\
\mathrm{MS}^{4}[163]: 109\end{array}$ & 5'-Hidroxijasmonic acid-5'-O-hexósido \\
\hline \multirow[t]{2}{*}{2} & 6.8 & 283,327 & 449 & $\begin{array}{l}\mathrm{MS}^{2}[449]: 287 ; \mathrm{MS}^{3}[287]: 151 ; \\
\mathrm{MS}^{4}[151]: 107\end{array}$ & Eriodictiol-O-hexósido \\
\hline & & & 507 & $\begin{array}{l}\mathrm{MS}^{2}[507]: 489(20), 471(10), 345(35) \\
327(100), 315(5) ; \mathrm{MS}^{3}[327]: \\
\text { 312(100), 167(20); } \mathrm{MS}^{3}[345]: \\
\text { 327(100), 315(15), 309(20), 287(5) }\end{array}$ & Quercetagetina dimetil eter $O$-hexósido \\
\hline 3 & 7.3 & 283,327 & 449 & $\begin{array}{l}\mathrm{MS}^{2}[449]: \text { 287(100), 269(<1), 151(1); } \\
\mathrm{MS}^{3}[287]: 269(4), 161(<1), 151(100) \\
\text { 125(4), 107(1); } \mathrm{MS}^{4}[151]: 107\end{array}$ & Eriodictiol-O-hexósido \\
\hline 4 & 8.6 & 248,342 & 447 & $\begin{array}{l}\mathrm{MS}^{2}[447]: 285(100) ; \mathrm{MS}^{3}[285]: \\
\text { 243(60), 241(100), 199(100), } \\
\text { 175(50), 151(10) }\end{array}$ & Luteolina-5-O- $\beta$-glucósido \\
\hline \multirow[t]{2}{*}{5} & 9.1 & 283,340 & 433 & $\begin{array}{l}\mathrm{MS}^{2}[433]: 271(100) ; \mathrm{MS}^{3}[271]: \\
227(1), 177(10), 151(100), 107(2)\end{array}$ & Naringenina-O- hexósido \\
\hline & & 283,327 & 463 & $\begin{array}{l}\mathrm{MS}^{2}[463]: 301(20), 287(100) \\
\mathrm{MS}^{3}[287]: 151(100), 135(<1), 125(<1)\end{array}$ & Eriodictiol-O- hexurónido \\
\hline \multirow[t]{2}{*}{6} & 9.6 & $\begin{array}{l}254,267 \\
345\end{array}$ & 461 & $\begin{array}{l}\mathrm{MS}^{2}[461]: 285(100) ; \mathrm{MS}^{3}[285]: \\
241(95), 217(60), 199(60), 175(60) \\
151(20)\end{array}$ & Luteolina-7- $\alpha$-O-glucurónido \\
\hline & & & 447 & $\begin{array}{l}\mathrm{MS}^{2}[447]: 285 ; \mathrm{MS}^{3}[285]: 243(50), \\
241(100), 199(60), 175(50), 151(15)\end{array}$ & Luteolina-7-O-glucósido \\
\hline 7 & 10.9 & 245,338 & 461 & $\begin{array}{l}\mathrm{MS}^{2}[461]: 446(1), 341(4), 323(3), \\
\text { 299(100); } \mathrm{MS}^{3}[299]: 284(100) ; \\
\mathrm{MS}^{4}[284]: 256(40), 151(5) ; \mathrm{MS}^{5}[256]: \\
\text { 239(4), 227(100), 211(20), 200(10), } \\
\text { 122(60), 94(2) }\end{array}$ & Crisoeriol-7- $\beta$-O-glucósido \\
\hline 8 & 11.3 & 267,332 & 445 & $\begin{array}{l}\mathrm{MS}^{2}[445]: 269(100) \\
175(5) ; \mathrm{MS}^{3}[269]: 225(5), 183(1)\end{array}$ & Apigenina-7- $\beta$-O-glucurónido \\
\hline 9 & 11.5 & 290,328 & 359 & $\begin{array}{l}\mathrm{MS}^{2}[359]: 223(15), 197(25), 179(30), \\
\text { 161(100), 133(4); } \mathrm{MS}^{3}[179]: 161(25) \\
151(<1), 135(100)\end{array}$ & Acido rosmarinic 0 \\
\hline 10 & 12.5 & 290,323 & 537 & $\begin{array}{l}\mathrm{MS}^{2}[537]: 493 ; \mathrm{MS}^{3}[493]: 359(100), \\
\text { 357(15), 313(10), 295(3), 269(<1), } \\
\text { 247(<1), 179(1), 161(1); } \mathrm{MS}^{4}[359]: \\
\text { 249(5), 223(10), 197(15), 179(25), } \\
\text { 161(100), 135(5) }\end{array}$ & Acido 3'-O-(8"'-Z-Caffeoyl) rosmarínico \\
\hline
\end{tabular}


Tabla 2 - Modificaciones químicas (ס) de los compuestos fenólicos 4, 6, 7, 8 y 9 (en DMSO-d $\mathrm{d}_{6}$ ) del extracto de T. $x$ citriodorus.

\begin{tabular}{|c|c|c|c|c|c|c|c|c|c|c|c|}
\hline \multirow{3}{*}{$\begin{array}{c}\text { Compuesto } \\
\text { Atomo }\end{array}$} & \multirow{2}{*}{\multicolumn{2}{|c|}{$\begin{array}{l}4 \\
\text { lina-5- } \beta-O- \\
\text { ucósido }\end{array}$}} & \multirow{2}{*}{\multicolumn{2}{|c|}{$\begin{array}{l}6 \\
\text { ina-7- } \alpha-0 \\
\text { urónido }\end{array}$}} & \multirow{2}{*}{\multicolumn{2}{|c|}{$\begin{array}{c}7 \\
\text { Crisoeriol-7- } \beta-O- \\
\text { glucósido }\end{array}$}} & \multirow{2}{*}{\multicolumn{2}{|c|}{$\begin{array}{l}8 \\
\text { nina-7- } \beta-O- \\
\text { curónido }\end{array}$}} & \multirow{2}{*}{\multicolumn{3}{|c|}{$\begin{array}{c}9 \\
\text { Ácido Rosmarínico }\end{array}$}} \\
\hline & & & & & & & & & & & \\
\hline & $\begin{array}{l}{ }^{13} \mathbf{C} \\
(\mathrm{ppm})\end{array}$ & $\begin{array}{c}{ }^{1} \mathbf{H} \\
(\mathrm{ppm})\end{array}$ & $\begin{array}{l}{ }^{13} \mathbf{C} \\
(\mathrm{ppm})\end{array}$ & $\begin{array}{c}{ }^{1} \mathbf{H} \\
(\mathrm{ppm})\end{array}$ & $\begin{array}{l}{ }^{13} \mathbf{C} \\
(\mathrm{ppm})\end{array}$ & $\begin{array}{c}{ }^{1} \mathbf{H} \\
(\mathrm{ppm})\end{array}$ & $\begin{array}{l}{ }^{13} \mathbf{C} \\
(\mathrm{ppm})\end{array}$ & $\begin{array}{c}{ }^{1} \mathbf{H} \\
(\mathrm{ppm})\end{array}$ & $\begin{array}{l}\text { Ato } \\
\text { mo }\end{array}$ & $\begin{array}{l}{ }^{13} \mathbf{C} \\
(\mathrm{ppm})\end{array}$ & $\begin{array}{c}{ }^{1} \mathbf{H} \\
(\mathrm{ppm})\end{array}$ \\
\hline 2 & 161.2 & - & 164.5 & - & 160.8 & - & 164.3 & - & 1 & 130.4 & - \\
\hline 3 & 105.3 & $6.50(\mathrm{~s})$ & 103.1 & $6.76(\mathrm{~s})$ & 106.2 & $6.58(\mathrm{~s})$ & 103.1 & $6.88(\mathrm{~s})$ & 2 & 114.5 & 6.65 (br s) \\
\hline 4 & 176.6 & - & 182.0 & - & 176.1 & - & ni & - & 3 & 144.7 & $\mathrm{ni}$ \\
\hline 5 & 158.7 & - & 161.2 & $13.00(\mathrm{~s})$ & ni & $12.97(\mathrm{~s})$ & ni & ni & 4 & 143.5 & ni \\
\hline 6 & 105.2 & $6.73(\mathrm{br} \mathrm{s})$ & 99.5 & $6.45(\mathrm{br} \mathrm{s})$ & 99.5 & 6.43 (br s) & 104.4 & $\begin{array}{l}6.81(\mathrm{~J} \\
2.0 \mathrm{~Hz})\end{array}$ & 5 & 116.4 & $\begin{array}{c}6.59(\mathrm{~d}, J \\
7.6 \mathrm{~Hz})\end{array}$ \\
\hline 7 & ni & $\begin{array}{c}\mathrm{OH} \\
8.45(\mathrm{~s})\end{array}$ & 162.8 & - & ni & ni & $\mathrm{ni}$ & ni & 6 & 120.0 & $\begin{array}{c}6.47(\mathrm{br} \mathrm{d}, \\
J 7.6 \mathrm{~Hz})\end{array}$ \\
\hline 8 & 98.5 & $6.61(\mathrm{br} \mathrm{s})$ & 94.6 & $6.80(\mathrm{br} \mathrm{s})$ & 94.6 & 6.83 (br s) & 98.1 & $\begin{array}{l}6.73(\mathrm{~J} \\
2.0 \mathrm{~Hz})\end{array}$ & 7 & 36.3 & $\begin{array}{c}3.00(\mathrm{~d}, \mathrm{~J} \\
12.4 \mathrm{~Hz})\end{array}$ \\
\hline 9 & $\mathrm{ni}$ & - & 157.1 & - & $\mathrm{ni}$ & - & $\mathrm{ni}$ & - & 8 & 76.2 & $\begin{array}{l}2.70(\mathrm{~d}, J \\
12.4 \mathrm{y} \\
4.79(\mathrm{br} \mathrm{d}, \\
J 10.1 \mathrm{~Hz})\end{array}$ \\
\hline 10 & 107.4 & - & 105.4 & - & ni & - & ni & - & 9 & ni & ni \\
\hline $1^{\prime}$ & 121.1 & - & 121.4 & - & 123.2 & - & ni & - & $1^{\prime}$ & 125.7 & - \\
\hline $2^{\prime}$ & 112.9 & 7.34 (s) & 113.6 & $\begin{array}{c}7.43(\mathrm{br} \\
\mathrm{d})\end{array}$ & 112.8 & 7.40 (br s) & 128.4 & $\begin{array}{c}7.97(\mathrm{~d}, J \\
8.7 \mathrm{~Hz})\end{array}$ & $2^{\prime}$ & 114.5 & $7.02(\mathrm{br} \mathrm{s})$ \\
\hline $3^{\prime}$ & 145.8 & $\mathrm{OH}$ & 145.8 & $\begin{array}{c}-9.48(b r \\
\mathrm{s})\end{array}$ & 146.8 & - & 116.0 & $\begin{array}{l}6.94(\mathrm{~d}, J \\
8.7 \mathrm{~Hz})\end{array}$ & $3^{\prime}$ & 148.2 & ni \\
\hline $4^{\prime}$ & 149.8 & $\mathrm{OH}$ & 150.0 & 10.01 & 150.7 & ni & 161.5 & - & $4^{\prime}$ & 145.7 & ni \\
\hline $5^{\prime}$ & 115.8 & $\begin{array}{c}512(\mathrm{hr} c) \\
6.85(\mathrm{~d}, J \\
7.8 \mathrm{~Hz})\end{array}$ & 115.9 & $\begin{array}{c}(\mathrm{hr} \mathrm{cl} \\
6.90(\mathrm{~d}, J \\
8.4 \mathrm{~Hz})\end{array}$ & 110.5 & $\begin{array}{c}7.07(\mathrm{~d}, J \\
8.2 \mathrm{~Hz})\end{array}$ & $\mathrm{ni}$ & ni & $5^{\prime}$ & 115.4 & $\begin{array}{c}6.74(\mathrm{~d}, J \\
8.4 \mathrm{~Hz})\end{array}$ \\
\hline $6^{\prime}$ & 118.6 & $\begin{array}{c}7.35(\mathrm{~d}, J \\
7.8 \mathrm{~Hz})\end{array}$ & 119.2 & $\begin{array}{c}7.45(\mathrm{~d}, J \\
8.4 \mathrm{~Hz})\end{array}$ & 118.4 & $\begin{array}{c}7.49(\mathrm{br} \mathrm{d}, \\
J 8.2 \mathrm{~Hz})\end{array}$ & ni & $\mathrm{ni}$ & $6^{\prime}$ & 121.0 & $\begin{array}{c}6.94(\mathrm{~d}, J \\
8.4 \mathrm{~Hz})\end{array}$ \\
\hline Sugar & & & & & & & & & $7^{\prime}$ & 143.5 & $\begin{array}{c}7.34(\mathrm{~d}, J \\
15.9 \mathrm{~Hz})\end{array}$ \\
\hline $1^{\prime \prime}$ & 105.1 & $\begin{array}{c}4.67(\mathrm{~d}, J \\
7.3 \mathrm{~Hz})\end{array}$ & 99.5 & $5.19(\mathrm{br} \mathrm{s})$ & 103.1 & $\begin{array}{c}4.68(\mathrm{~d}, J \\
7.0 \mathrm{~Hz})\end{array}$ & 99.5 & $\begin{array}{c}5.03(\mathrm{~d}, J \\
7.3 \mathrm{~Hz})\end{array}$ & $8^{\prime}$ & 115.1 & $\begin{array}{c}6.16(\mathrm{~d}, J \\
15.9 \mathrm{~Hz})\end{array}$ \\
\hline $2^{\prime \prime}$ & 73.6 & & 73.0 & * & ni & ni & ni & ni & $9^{\prime}$ & 166.2 & \\
\hline $3^{\prime \prime}$ & 75.6 & * & 75.2 & * & ni & ni & ni & ni & & & \\
\hline $4^{\prime \prime}$ & 69.8 & * & 72.9 & * & ni & ni & ni & ni & & & \\
\hline $5^{\prime \prime}$ & 77.6 & * & 72.8 & * & ni & - & ni & - & & & \\
\hline $6^{\prime \prime}$ & 60.8 & * & $\mathrm{ni}$ & - & ni & ni & ni & ni & & & \\
\hline
\end{tabular}

* Abajo del pico del agua

ni - no identificado 
En cuanto a las flavonas detectadas como compuestos mayoritarios en el extracto T. $x$ citriodorus, se encontraron tres derivados de la luteolina, que se eluían con las fracciones 4 (ion $[\mathrm{M}-\mathrm{H}]^{-}$con $\mathrm{m} / \mathrm{z} 447$ ) y 6 (iones $[\mathrm{M}-\mathrm{H}]^{-} 461$ y 447)). El ultimo ion fue determinado como luteolina-7-O-glucósido, mientras el que eluía en la fracción 4 fue identificado como luteolina-5- $\beta$-O-glucósido, basandonos en los datos espectrales de 1D y 2D NMR (Table 2). Este compuesto había sido detectado en extractos de las plantas $T$. sipyleus y en $T$. praecox. Además, el análisis por HPLC-DAD-ESI-MS ${ }^{n}$ en conjunto con NMR permitió asignar como compuesto principal de la fracción 6 la luteolina-7- $\alpha$-O-glucurónido. Para plantas del genero Thymus plantas solo se habían descrito 7-O- $\beta$-isomeros de este compuesto. El extracto de T. $x$ citriodorus contiene también en su fracción 8 (ion $[\mathrm{M}-\mathrm{H}]^{-}$con $\mathrm{m} / \mathrm{z} 445$ ) el derivado 7- $\beta$-O-glucurónido de la flavona apigenina que había sido determinada previamente en otras especies vegetales de Thymus. También se encontró por primera vez en plantas del genero Thymus otra flavona, el crisoeriol-7- $\beta$-O-glucósido.

En cuanto a las flavanonas, la fragmentación obtenida para la fracción 1 (ion $[\mathrm{M}-\mathrm{H}]^{-}$ con $\mathrm{m} / \mathrm{z}$ 611) sugirió un di-O-hexósido del eriodictiol. Otros tres derivados glicosilo de esta flavanona han sido determinados en este trabajo y incluyen dos eriodictiol- $O$ hexósido (fracciones 2 y 3) y el eriodictiol-O-hexurónido (fracción 5). Además de estos derivados otra flavanona, la naringenin-O-hexósido, ha sido identificada en el extracto en la fracción 5 (con $\mathrm{m} / \mathrm{z}$ de 433).

A semejanza de lo que esta descrito en otros estudios sobre el género Thymus, el extracto estudiado en este trabajo se mostró poco abundante en flavonoles. De hecho, se propone que el compuesto que eluye en la fracción 2 sea quercetagetina-dimetiléter-O-hexósido ( $\mathrm{m} / \mathrm{z} 507)$ que aparece como compuesto minoritario en esa fracción.

En lo que respecta a la cuantificación de los compuestos determinados, y al igual que en otras especies de Thymus, el extracto etanólico de T. $x$ citriodorus contiene grandes cantidades de ácido rosmarínico $(10,4 \pm 0,6 \mathrm{mg} / \mathrm{g}$ de extracto), sin embargo otros fenólicos menos descritos en plantas del mismo género se detectaron como compuestos fenólicos abundantes en el extracto etanólico de T. $x$ citriodorus y incluyen la luteolina-7-O- $\alpha$-glucurónido (12 $\pm 2 \mathrm{mg} / \mathrm{g}$ de extracto) y la apigenina-7-O- $\beta$ glucurónido ( $9 \pm 2 \mathrm{mg} / \mathrm{de}$ extracto).

El método de HPLC-DAD usado para la cuantificación de los compuesto fenólicos en T. $x$ citriodorus ha demostrado buena linealidad para los compuestos de referencia probadas y también valores satisfactorios de repetibilidad y de precisión para el instrumento y para el método. Por otra parte, los resultados satisfactorios de análisis Pereira O. R., 2013 | CARACTERIZACION DE COMPUESTOS FENÓLICOS EN PLANTAS MEDICINALES Y EVALUACIÓN DE ACTIVIDADES FARMACOLÓGICAS: PROPIEDADES ANTIOXIDANTES 
de la precisión intermedia y ensayos de recuperación indicaron que el método cromatográfico puede ser utilizado para cuantificar los principales compuestos fenólicos de T. $x$ citriodorus con precisión y exactitud adecuadas.

Como conclusión, esta primera parte del trabajo surge como una contribución importante una vez que describe por primera vez los componentes fenólicos de la especie T. $x$ citriodorus. Además, las técnicas utilizadas permitieron detectar por primera vez en el género Thymus, compuestos como el eriodictiol-di-O-hexósido, el crisoeriol-7-O-glucósido, la quercetagetina-dimetil-éter-O-hexósido y la naringenina-Ohexósido. 


\subsubsection{Identificación de compuestos fenólicos de Cytisus multiflorus}

Cytisus multiflorus es una planta de la familia Fabaceae conocida por sus propiedades beneficiosas que muchas veces se asocian con su contenido en compuestos fenólicos. Sin embargo, esta especie está menos estudiada que otras del mismo género botánico $y$, hasta donde sabemos, su perfil fenólico sigue desconocido. En este contexto, esta parte del trabajo tuvo por objetivo caracterizar la composición fenólica del extracto etanólico obtenido de la planta medicinal Cytisus multiflorus.

Como se puede verificar en la Tabla 1, que resume los datos obtenidos por HPLCDAD y MS para cada una de las fracciones recogidas por HPLC (Fig. 1), C. multiflorus era principalmente abundante en flavonas. Además de este grupo de compuestos, sólo se encontró un derivado de quercetina (flavonol) en el extracto. El total de fenólicos determinados en el extracto de C. multiflorus por HPLC es de 41,8 $\pm 3,0 \mathrm{mg} / \mathrm{g}$ planta seca. En más detalle, los derivados de la crisina aparecieron en las fracciones 9, 10 y 12. El compuesto crisina-7-O- $\beta$-D-glucopiranósido (MW 452 Da, fracción 9), cuya estructura fue confirmada para NMR, en conjunto con el isómero de la crisina (fracción 10) representaron los compuestos mayoritario en el extracto de $C$. multiflorus $(15,9 \pm 2,3$ y $7,0 \pm 1,3 \mathrm{mg} / \mathrm{g}$ planta seca, respectivamente).

Tabla 1 - Identificación y cuantificación de las fracciones de Cytisus multiflorus eluidas por HPLC.

\begin{tabular}{|c|c|c|c|c|c|}
\hline Fracción & $\begin{array}{l}\mathbf{R T} \\
(\min )\end{array}$ & $\lambda_{\max }$ & $\begin{array}{l}\text { MW } \\
(\mathrm{Da})\end{array}$ & Compuesto & $\begin{array}{l}\mathrm{mg} / \mathrm{g} \text { planta } \\
\text { seca }\end{array}$ \\
\hline 1 & 7.2 & $\begin{array}{l}256,266 \\
347\end{array}$ & 580 & $\begin{array}{c}\text { 2"-O-pentosilo-6- } C \text {-hexosilo- } \\
\text { luteolina }\end{array}$ & $3,3 \pm 0,5$ \\
\hline 2 & 7.5 & $\begin{array}{l}257,266 \\
\quad 346\end{array}$ & 580 & $\begin{array}{c}\text { 2"-O-pentosilo-8-C-hexosilo- } \\
\text { luteolina }\end{array}$ & $3,5 \pm 0,3$ \\
\hline 3 & 7.9 & $\begin{array}{l}256,266 \\
\quad 345\end{array}$ & 448 & Orientina & $0,8 \pm 0,1$ \\
\hline 4 & 8.1 & 267,338 & 564 & $\begin{array}{c}\text { 2"-O-pentosilo-8- } C \text {-hexosilo- } \\
\text { apigenina }\end{array}$ & $0,5 \pm 0,1$ \\
\hline 5 & 8.3 & 267,338 & 564 & $\begin{array}{c}\text { 2"-O-pentosilo-6- } C \text {-hexosilo- } \\
\text { apigenina }\end{array}$ & $0,9 \pm 0,1$ \\
\hline \multirow[t]{2}{*}{6} & 9.3 & 255,352 & 610 & Rutina & $4,5 \pm 0,7$ \\
\hline & & & 448 & Luteolina-5-O-glucósido & $3,6 \pm 0,7$ \\
\hline
\end{tabular}




\begin{tabular}{|c|c|c|c|c|c|}
\hline & & & 724 & $\begin{array}{l}\text { 6"-O-(3-hidroxi-3- } \\
\text { metilglutaroilo)-2"-O-pentosilo- } \\
\text { C-hexosilo-luteolina }\end{array}$ & $0,8 \pm 0,1$ \\
\hline \multirow{3}{*}{7} & \multirow{3}{*}{9.7} & 266,342 & 708 & $\begin{array}{l}\text { 6"-O-(3-hidroxi-3- } \\
\text { metilglutaroilo)-2"-O-pentosilo- } \\
\text { C-hexosilo-apigenina }\end{array}$ & $11,2 \pm 2,1$ \\
\hline & & & 464 & Quercetina-3-O-glucósido & \\
\hline & & $\begin{array}{c}255,262 \\
347\end{array}$ & 448 & Luteolina-7-O-glucósido & \\
\hline 8 & 11.3 & 266,342 & 432 & Apigenin-7-O-glucósido & $0,8 \pm 0,1$ \\
\hline \multirow{2}{*}{9} & \multirow{2}{*}{14.3} & \multirow{2}{*}{267,303} & 462 & \multirow{2}{*}{$\begin{array}{l}\text { Crisina-7-O- } \beta \text {-D- } \\
\text { glucopiranósido }\end{array}$} & \multirow{2}{*}{$15,9 \pm 2,3$} \\
\hline & & & 452 & & \\
\hline 10 & 15.7 & 267,303 & 254 & Isomero de Crisina & $7,0 \pm 1,3$ \\
\hline 11 & 16.7 & - & 270 & Apigenina & $0,5 \pm 0,1$ \\
\hline 12 & 23.7 & 267,313 & 254 & Crisina & $0,5 \pm 0,1$ \\
\hline
\end{tabular}

Medias \pm SD

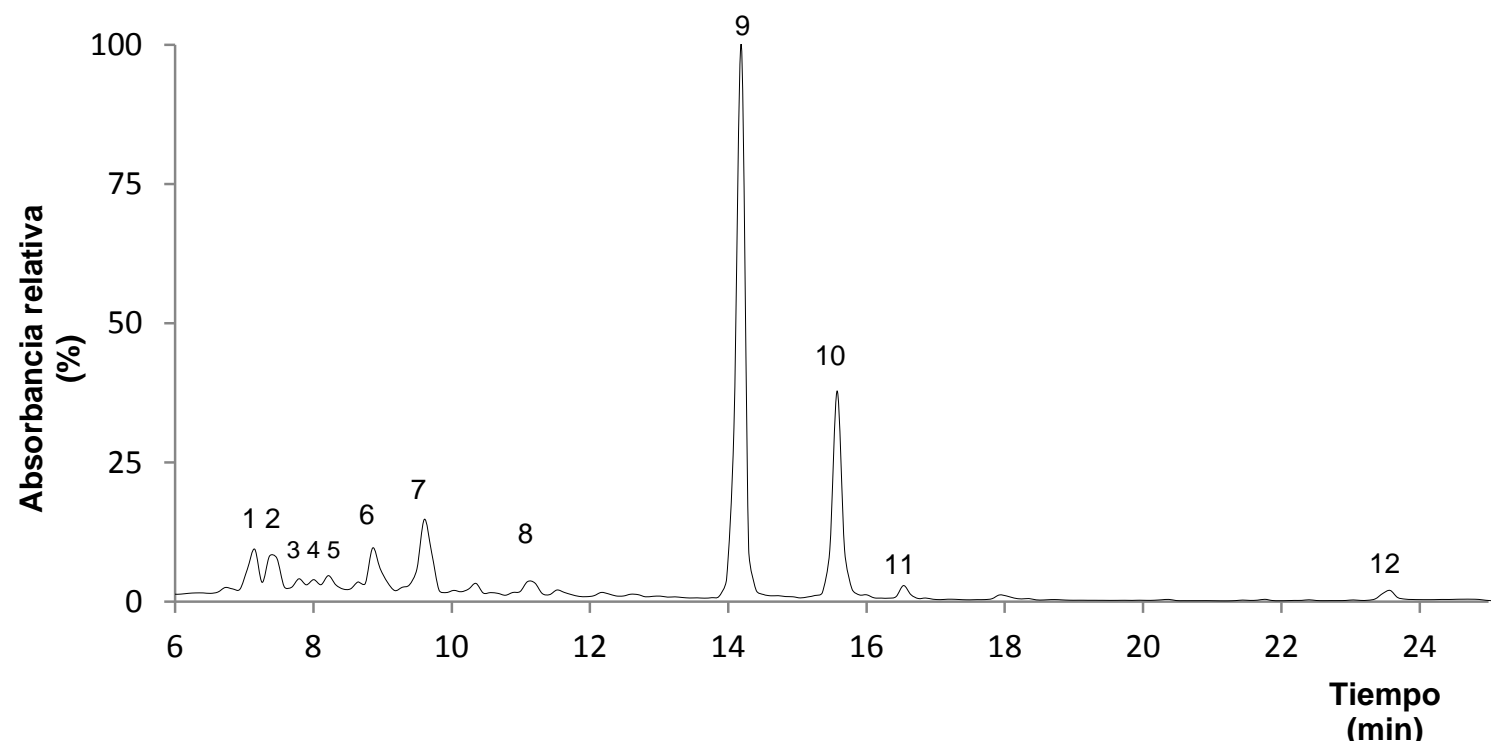

Figure 1 - Perfil cromatográfico del extracto etanólico de Cytisus multiflorus obtenido por HPLC a $280 \mathrm{~nm}$. 
Como se puede verificar en la Tabla 1 y la Fig. 1, además de derivados de la crisina, el extracto contenía el flavonol rutina $\left(4,5 \pm 0,7 \mathrm{mg} / \mathrm{g}\right.$ planta seca) $\left([\mathrm{M}-\mathrm{H}]^{-}\right.$con $\left.\mathrm{m} / \mathrm{z} 609\right)$ y otros compuestos como 2"-O-pentosilo-6-C-hexosilo-luteolina, 2"-O-pentosilo-8- $C$ hexosilo-Iuteolina, y $\quad$ 6"-O-(3-hidroxi-3-metilglutaroilo)-2"-O-pentosilo- $C$-hexosiloapigenina que no están muy descritos en la familia Fabaceae. Como compuestos minoritarios, se determinaron en el extracto otras flavonas como la común crisina (facción 12) $\left([\mathrm{M}-\mathrm{H}]^{-}\right.$con $\mathrm{m} / \mathrm{z}$ 253) y derivados de la luteolina con $[\mathrm{M}-\mathrm{H}]^{-}$a $m / z 447$, como el $C$-glucósido orientina, la luteolina-5-O-glucósido y la luteolina-7-O-glucósido. Además, las flavonas apigenina y apigenina-7-O-glucósido se identificaron en el extracto basándonos en que su tiempo de retención, espectro UV-Vis y la fragmentación obtenida en los experimento de MS, coincidían con los de compuestos patrón. También es importante decir, que en este trabajo se han determinado nuevos compuestos fenólicos de que son ejemplo el 2"-O-pentosilo-6- $C$-hexosilo-apigenina, 2"-O-pentosilo-8-C-hexosilo-apigenina y el 6"-O-(3-hidroxi-3-methilglutaroil)-2"-Opentosilo- $C$-hexosilo-luteolina.

En general, esta parte del trabajo surge como una contribución valiosa para la dilucidación de los compuestos fenólicos presentes en el género Cytisus y en la familia Fabaceae. 


\subsubsection{Compuestos fenólicos de Lamium album L.: derivados de isoscutelareína}

Lamium album L., más conocida como ortiga blanca, es una planta mediterránea herbácea perenne utilizada como alimento, en suplementos alimenticios y en forma de infusión. Esta especie vegetal se viene utilizando desde hace décadas en la medicina tradicional para el tratamiento de varias enfermedades, sin embargo, el conocimiento de su constitución en fitoquímicos es escaso. En este sentido, el objetivo de esta parte del trabajo, fue la caracterización detallada de la composición fenólica del extracto etanólico purificado de L. album.

Como se puede observar en la Fig. 1 y en la Tabla 1, el extracto de $L$. album estaba constituido por flavonas, feniletanóides y una flavanona. De estos compuestos, hay que resaltar a los derivados de la flavona isoscutelareína puesto que, para el género Lamium, se detectaron por primera vez en este estudio.

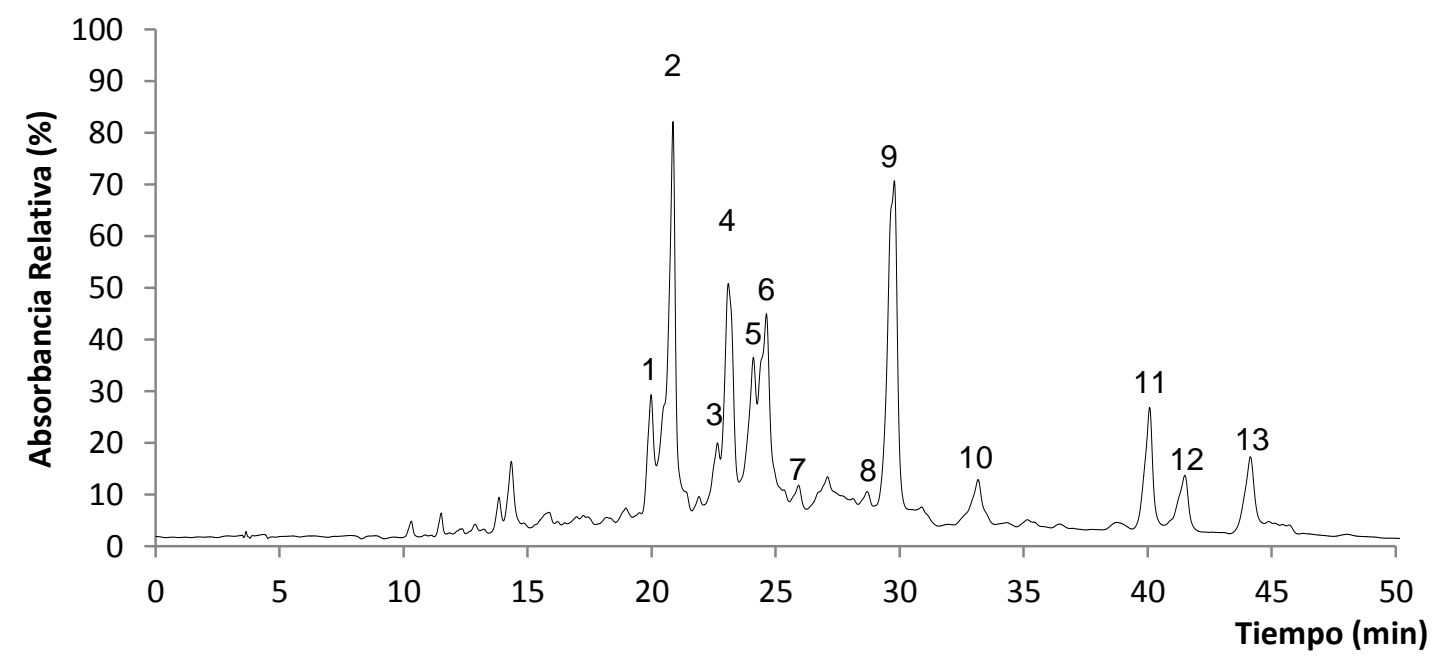

Figure 1 - Perfil cromatográfico del extracto purificado de Lamium album L. registrado a 340 $\mathrm{nm}$. 
Tabla 1 - Identificación y cuantificación de las fracciones de Lamium album eluidas por HPLC.

\begin{tabular}{|c|c|c|c|c|c|}
\hline Fracción & $\begin{array}{l}\text { Tiempo } \\
\text { retención } \\
(\mathrm{min})\end{array}$ & $\lambda \max$ & {$[\mathrm{M}-\mathrm{H}]^{-}$} & Compuesto & mg/g extracto \\
\hline 1 & 20.0 & $254,267,345$ & - & Derivado de Luteolina & - \\
\hline 2 & 20.9 & 290,329 & 623 & Verbascósido & $233,7 \pm 13,6$ \\
\hline 3 & 22.7 & 290,328 & 623 & Isoverbascósido & $39,2 \pm 5,6$ \\
\hline 4 & 23.1 & $275,302,333$ & 609 & $\begin{array}{l}\text { Isoscutelareina-7-O- } \\
\text { alosilo( } 1 \rightarrow 2) \text { glucósido }\end{array}$ & $26,8 \pm 5,3$ \\
\hline 5 & 24.1 & $275,302,333$ & 651 & $\begin{array}{l}\text { Isoscutelareina-7-O-(6-O- } \\
\text { acetilalosilo })(1 \rightarrow 6) \text { glucósido }\end{array}$ & $23,6 \pm 6,7$ \\
\hline 6 & 24.6 & $254,267,345$ & 447 & Luteolina-7-O-glucósido & $29,7 \pm 2,2$ \\
\hline 7 & 25.9 & $275,302,333$ & 651 & $\begin{array}{c}\text { Isoscutelareina-7-O-(6-O- } \\
\text { acetilalosilo }(1 \rightarrow 2) \text { glucósido } \\
\text { isomer }\end{array}$ & $9,6 \pm 0,3$ \\
\hline 8 & 28.7 & 266,342 & 431 & Apigenina-7-O-glucósido & $16,1 \pm 5,8$ \\
\hline 9 & 29.8 & $275,302,333$ & 651 & $\begin{array}{l}\text { Isoscutelareina-7-O-(6-O- } \\
\text { acetilalosilo }(1 \rightarrow 2) \text { glucósido }\end{array}$ & $37,4 \pm 4,4$ \\
\hline 10 & 33.2 & $275,305,327$ & 623 & $\begin{array}{l}\text { 4'-O-Metilisoscutelarein-7-O- } \\
\text { alosilo }(1 \rightarrow 2) \text { glucósido }\end{array}$ & $16,6 \pm 6,5$ \\
\hline 11 & 40.1 & $275,305,327$ & 665 & $\begin{array}{l}\text { 4'-O-Metilisoscutelarein-7-O- } \\
\text { (6-O-acetilalosil })(1 \rightarrow 2) \\
\text { glucósido }\end{array}$ & $19,4 \pm 5,2$ \\
\hline 12 & 41.5 & 266, 342 & 577 & Apigenina-7-O-rutinósido & $16,2 \pm 4,7$ \\
\hline 13 & 44.2 & - & 579 & Naringenina-7-O-rutinósido & $32,6 \pm 5,6$ \\
\hline
\end{tabular}

El extracto de $L$. album es principalmente abundante en dos feniletanoides que eluían en las fracciones 2 y 3 (Fig. 1) que son el verbascósido y el isoverbascósido y contienen $233,7 \pm 13,6$ y $39,2 \pm 5,6 \mathrm{mg} / \mathrm{g}$ de extracto, respectivamente. Los dos isómeros con peso molecular de $624 \mathrm{Da}$ corresponden a aproximadamente la mitad de los fenoles totales determinados $(500.7 \pm 50.0 \mathrm{mg} / \mathrm{g}$ de extracto). Además, el extracto contenía también compuestos fenólicos bioactivos poco comunes, catalogados en su conjunto, como derivados de la isoscutelareína, que representaban aproximadamente el $30 \%$ del total los compuestos fenólicos determinados (Tabla 1). Estos compuestos, que eluyeron en las fracciones 4, 5, 7, 9, 10 y 11, mostraron espectros UV, con máximos a 278, 302 y $333 \mathrm{~nm}$, que está de acuerdo con lo descrito en la literatura para glucósidos isoscuttelareina. De entre todos, el principal derivado determinado fue la isoscutelareina-7-O-(6-O-acetil- $\beta$-alosilo)(1 $\rightarrow 2)-\beta$-glucósido (MW 652 Da) que 
representaba $37,4 \pm 4,4 \mathrm{mg} / \mathrm{g}$ de extracto de $L$. album. Su estructura ha sido en primer lugar determinada por HPLC y $\mathrm{MS}^{n}$ y posteriormente confirmada por experimentos de ${ }^{1} \mathrm{H}$ y ${ }^{13} \mathrm{C}$ NMR cuyos resultados coincidieron con lo estaba descrito en la literatura. Otros derivados de la flavona incluyen el compuesto isoscutelareina-7-Oalosilo( $1 \rightarrow 2$ )glucósido (ion $[\mathrm{M}-\mathrm{H}]^{-}$con $\mathrm{m} / \mathrm{z} 609$ ), su derivado $O$-metilo $\left([\mathrm{M}-\mathrm{H}]^{-}\right.$ion a $\mathrm{m} / \mathrm{z}$ 623), derivados acetilo de la isoscutelareína-O-alosilo glucósido (fracciones 5 y 7 ) y un derivados acetilo del compuesto O-metilisoscutelareína-7-O-alosilo( $1 \rightarrow 2)$ glucósido (MW $666 \mathrm{Da}$ ). Además de los compuestos principales, el extracto contiene menores cantidades de las flavonas apigenina-7-O-glucósido, luteolina-7-O-glucósido y apigenina-7-O-rutinósido. La única flavanona identificada fue la naringenina-7-Orutinósido (MW $580 \mathrm{Da})$.

En conclusión, esta parte del trabajo es un importante estudio acerca la caracterización química de la especie vegetal $L$. album, sugiriendo la especie como una importante fuente dietética de antioxidantes naturales. No obstante, se necesitan más estudios para aclarar la contribución exacta de los compuestos fenólicos en los efectos beneficiosos en la salud humana que se proponen para esta planta. 


\subsubsection{Caracterización fenólica de extractos de Leonurus cardiaca L.}

Leonurus cardiaca L. (agripalma), subfamilia Lamioideae (Lamiaceae) es una planta originaria de Europa central, pero difundida en distintos países templados de todo el mundo. En la medicina tradicional se incluye en preparaciones internas y externas para el tratamiento de varias enfermedades. Sin embargo, sus propiedades beneficiosas (por ejemplo antioxidante, cardioprotectora y neuroprotectora) y los compuestos responsables por estos efectos han sido poco estudiados. Por ello, esta parte del trabajo tenía como objetivo investigar la composición fenólica de un extracto etanólico purificado de L. cardiaca así como evaluar sus propiedades antioxidantes.

El extracto de L. cardiaca es abundante en compuestos fenólicos con $15 \mathrm{mg} / \mathrm{g}$ de planta seca $(500,4 \pm 49,1 \mathrm{mg} / \mathrm{g}$ de extracto) como determinado por HPLC-DAD (Tabla 1. De entre los cuantificados, los más abundantes son los feniletanoides glucósidos lavandulifolioside (MW $756 \mathrm{Da}$ ) y verbascósido que representan el 50\% y el 27\%, respectivamente (Tabla 1 ).

Tabla 1 - Identificación de las fracciones del extracto etanólico de Lamium album L. que eluyeron por HPLC.

\begin{tabular}{|c|c|c|c|c|c|}
\hline Fracción & $\begin{array}{c}\text { Tiempo retención } \\
\text { (min) }\end{array}$ & $\lambda \max$ & {$[\mathrm{M}-\mathrm{H}]^{-}$} & Compuesto & $\mathrm{mg} / \mathrm{g}$ extracto \\
\hline \multirow{2}{*}{1} & \multirow{2}{*}{8,2} & 290,329 & 341 & Ácido cafeico glucósido & $3,7 \pm 0,8$ \\
\hline & & ND & 771 & Rutina-O-glucósido & ND \\
\hline 2 & 13,7 & ND & 625 & Quercetina-3-O-soforósido & $5,7 \pm 1,1$ \\
\hline 3 & 16,7 & 290,329 & 755 & Lavandulifoliósido & $253,6 \pm 35,8$ \\
\hline 4 & 17,5 & 290, 329 & 623 & Verbascósido & $137,4 \pm 19,9$ \\
\hline \multirow[t]{2}{*}{5} & 18,0 & $256,267,355$ & 609 & Rutina & $15,8 \pm 2,1$ \\
\hline & & $256,267,357$ & 463 & Quercetina-3-O-glucósido & $24,9 \pm 3,8$ \\
\hline \multirow[t]{2}{*}{6} & 18,9 & ND & 507 & Derivado del Ácido Cafeico & ND \\
\hline & & ND & 593 & Luteolina-7-O-rutinósido & ND \\
\hline 7 & 19,4 & ND & 637 & Leucoseptósido A & $31,5 \pm 4,6$ \\
\hline 8 & 20,0 & ND & 783 & Leonósido B & $25,1 \pm 4,7$ \\
\hline
\end{tabular}

Medias $\pm S D$

ND- No determinado 
Aunque menos representativos $(\sim 10 \%$ del total de fenólicos) los compuestos leucoseptósido A (con la fragmentación $637 \rightarrow 461 \rightarrow 315$ ) y leonósido $B$ $(783 \rightarrow 607 \rightarrow 475 \rightarrow 329)$ fueron detectados por primera vez en extractos de la especie vegetal $L$. cardiaca (fracciones 7 y 8 ). Una vez que la fragmentación de estos dos compuestos está muy poco descrita en la literatura, su estructura se determinó esencialmente a través de la interpretación de los datos de ESI-MS y MS ${ }^{n}$. De este modo, el espectro MS/MS del ion molecular con $\mathrm{m} / \mathrm{z} 637$ (fracción 7) demostró que el ion de $\mathrm{m} / \mathrm{z} 315$, que corresponde a una unidad (3,4 dihidroxifenilo)-glucopiranosilo, se formó a través de la pérdida de $176 \mathrm{Da}$ (ion a $\mathrm{m} / \mathrm{z} 461$ ) y $146 \mathrm{Da}$ (ion a $\mathrm{m} / \mathrm{z} 315$ ), lo que indica la pérdida de grupos feruloilo y ramnopiranosilo, respectivamente. Por otra parte, el ion molecular de la fracción 8 (ion a $m / z 783$ ), designado aquí como leonósido B presenta una fragmentación en la que se produce una pérdida de $176 \mathrm{Da}$ (ion con $\mathrm{m} / \mathrm{z} 607$ ) y $132 \mathrm{Da}$ (ion con $\mathrm{m} / \mathrm{z} 651$ ), atribuido a pérdidas de unidades feruloilo y arabinopiranosilo, respectivamente. Además, el espectro de $\mathrm{MS}^{4}$ de este último ion indica la pérdida de una unidad ramnopiranosilo, relacionado con la formación del ion $[\mathrm{M}-\mathrm{H}-146]^{-}$a $\mathrm{m} / \mathrm{z} 329$, que corresponde a un fragmento (3-hidroxi,4-metoxifenilo)glucopiranosilo. Además de feniletanóides glucósidos, el extracto contiene compuestos flavonóides (10\%) y derivados del ácido cafeico, representado estos últimos cantidades residuales de su total de compuestos fenólicos. Los flavonóides detectados en el extracto incluyeron, en su gran mayoría, derivados glicosídicos de quercetina, o más concretamente la rutina-O-glucósido (MW $772 \mathrm{Da}$ ) y la quercetina-3-O-soforósido (MW $626 \mathrm{Da}$ ), descritos por primera vez en la especie $L$. cardiaca y también isoquercitrina (quercetina-3-O-glucósido) y rutina (quercetina-3-O-rutinósido), ya anteriormente detectados en la misma especie vegetal.

Además de la caracterización del extracto de L. cardiaca, el presente estudio permitió también estimar sus propiedades antioxidantes a través de ensayos químicos. La especie ha demostrado poseer una elevada capacidad antioxidante con valores de $\mathrm{EC}_{50}$ de $18,3 \pm 1,5$ y $94,7 \pm 7,0 \mu \mathrm{g} / \mathrm{mL}$ obtenidos en los ensayo del DPPH y del poder reductor, respectivamente.

En conjunto, los resultados de esta parte del trabajo permiten decir que la especie $L$. cardiaca es una excelente fuente de compuestos fenólicos y que posee una importante actividad antioxidante. Sin embrago, son necesarios más experimentos para confirmar la actividad antioxidante y determinar la contribución de los compuestos fenólicos para este efecto. 


\subsubsection{Actividades hepatoprotectora y de captura de ROS por Mentha aquatica L. y Lavandula dentata L.}

Mentha aquatica L. y Lavandula dentata L. son dos especies vegetales que pertenecen a la gran familia Lamiaceae y que se usan como especias en la fabricación de alimentos y bebidas. La especie $M$. aquatica se viene utilizando desde hace muchos años en la medicina tradicional para el tratamiento de la inflamación externa, en limpieza bucal y para el tratamiento de los dolores de garganta, mientras que la especie de $L$. dentata se utiliza en infusiones para tratar de la diabetes, resfriados y cólicas renales. En este contexto, esta parte del trabajo tenía como objetivo determinar la composición exacta de extractos fenólicos de las dos especies vegetales y también evaluar y sus efectos antioxidantes y citoprotectores.

Los principales compuestos fenólicos detectados en los extractos etanólicos purificados a partir de plantas de las especies $M$. aquatica y $L$. dentata determinados por de HPLC-DAD, y ESI-MS están representados en la Tabla 1. El total de compuestos fenólicos cuantificados representó $303 \pm 29$ y $94 \pm 4 \mathrm{mg} / \mathrm{g}$ de extracto, en extractos de $M$. aquatica y $L$. dentata, respectivamente (Tabla 1 ).

Tabla 1 - Cuantificación a $280 \mathrm{~nm}$ de los principales fenólicos de extractos de las especies vegetales $M$. aquatica y $L$. dentata.

\begin{tabular}{|c|c|c|c|c|}
\hline \multirow[b]{2}{*}{ Fracción } & \multicolumn{2}{|c|}{ M. aquatica } & \multicolumn{2}{|c|}{ L. dentata } \\
\hline & Compuesto & $\begin{array}{l}\mathrm{mg} / \mathrm{g} \mathrm{de} \\
\text { extracto }^{\mathrm{a}}\end{array}$ & Compuesto & $\begin{array}{l}\mathrm{mg} / \mathrm{g} \text { de } \\
\text { extracto }^{\mathrm{a}}\end{array}$ \\
\hline 1 & Eriodictiol-7-O-rutinósido & $\begin{array}{c}144,6 \pm 22,4 \\
(48 \%)\end{array}$ & & \\
\hline 2 & Luteolina-7-O-rutinósido & $\begin{array}{c}43,3 \pm 10,0 \\
(14 \%)\end{array}$ & & \\
\hline 3 & Naringenina-7-O-rutinósido & $\begin{array}{c}24,4 \pm 3,7 \\
(8 \%)\end{array}$ & Luteolina-7-O-glucurónido & $\begin{array}{c}26,2 \pm 4,0 \\
(28 \%)\end{array}$ \\
\hline 4 & Hesperitina-7-O-rutinósido & $\begin{array}{c}25,9 \pm 3,6 \\
(9 \%)\end{array}$ & & \\
\hline 5 & Acido rosmarínico & $\begin{array}{c}64,2 \pm 8,8 \\
(21 \%)\end{array}$ & Ácido Rosmarínico & $\begin{array}{c}67,8 \pm 6,7 \\
(72 \%)\end{array}$ \\
\hline 6 & & & $\begin{array}{l}\text { Apigenina-7-O- } \\
\text { (acetil)glucósido }\end{array}$ & * \\
\hline & Total & $302,5 \pm 28,7$ & & $93,9 \pm 4,1$ \\
\hline \multicolumn{5}{|c|}{ Medias $\pm S D$} \\
\hline \multicolumn{5}{|c|}{$\begin{array}{l}\text { aLos valores entre paréntesis se expresan como el porcentaje de compuestos fenólicos totales } \\
\text { determinados }\end{array}$} \\
\hline \multicolumn{5}{|c|}{ * Por debajo del límite de cuantificación } \\
\hline \multicolumn{5}{|c|}{$\begin{array}{c}\text { Pereira O. R., } 2013 \text { | CARACTERIZACION DE COMPUESTOS FENÓLICOS EN PLANTAS MEDICINALES Y } \\
\text { EVALUACIÓN DE ACTIVIDADES FARMACOLÓGICAS: PROPIEDADES ANTIOXIDANTES } \\
\text { Y ANTI-INFLAMATORIAS }\end{array}$} \\
\hline
\end{tabular}


Los extractos presentaron grandes cantidades de ácido rosmarínico, representando $64 \pm 2$ y $68 \pm 3 \mathrm{mg} / \mathrm{g}$ del extracto etanólico de $M$. aquatica y $L$. dentata, respectivamente. Este compuesto fue el principal compuesto determinado en el extracto de $L$. dentata, pero a la inversa, el extracto de $M$. aquatica contenía gran cantidad de otros

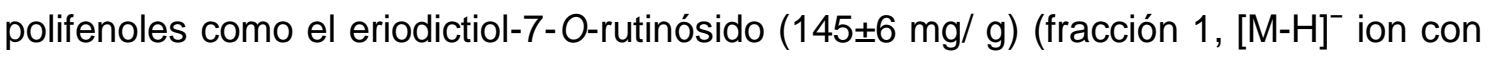
$m / z 595)$, lo que coincide con lo que esta descrito para otras especies de Mentha. De acuerdo con datos de la literatura para género Mentha, también se detectaron derivados glucósido, glucurónido y rutinósido de flavonas en el extracto de $M$. aquatica. En particular, se detectó la luteolina-7-O-glucósido y la apigenina-7-Orutinósido en cantidades muy pequeñas en los picos 3 y 4 , respectivamente. En cambio, el presente extracto de $M$. aquatica se mostró abundante en la flavona luteolina-7-O-rutinósido $(4,9 \pm 1,1 \mathrm{mg} / \mathrm{g}$ de planta seca) y contenía cantidades considerables $(\sim 10 \%)$ de naringenina-7-O-rutinósido y de hesperitina-7-O-rutinósido. La luteolina-7-O-glucurónido también se detectó en el extracto etanólico de L. dentata, donde representa el $28 \%$ de los componentes fenólicos determinados totales.

En lo que respecta a efectos estudiados, ambos extractos demostraron capacidades antioxidantes significativas, determinadas por los ensayos del DPPH • y poder reductor, así como en un modelo de estrés químico inducido por dicromato potásico en células hepáticas HepG2. Concretamente, se encontraron valores de $E_{50}$ inferiores en el extracto de $M$. aquatica en los dos ensayos químicos usados. En el test del DPPH los valores de $\mathrm{EC}_{50}$ fueron de $8,1 \pm 1,3$ y $11,6 \pm 1,1 \mu \mathrm{g} / \mathrm{mL}$ mientras que en el test del poder reductor fueron de $51,9 \pm 12,6$ y $78,9 \pm 2,6 \mu \mathrm{g} / \mathrm{mL}$, para los extractos de $M$. aquatica y $L$. dentata, respectivamente. Los ensayos realizados en los experimentos con la línea celular indicaron que el extracto de $L$. dentata presenta mejor capacidad para neutralizar la formación de ROS inducida por dicromato potásico (DK) en las células HepG2. En este ensayo, la protección observada fue del 30\% cuando las células HepG2 fueron incubadas con $25 \mu \mathrm{M}$ de DK y $50 \mu \mathrm{g} / \mathrm{mL}$ de extracto de $L$. dentata (Fig. 1A). Parece ser que este efecto antioxidante no estaba directamente asociado a su capacidad citoprotectora. De hecho, fue el extracto de $M$. aquatica, y no el de $L$. dentata, el que demostró un efecto citoprotector más eficaz (13\%), medido por el ensayo de MTT (Fig. 1B). 

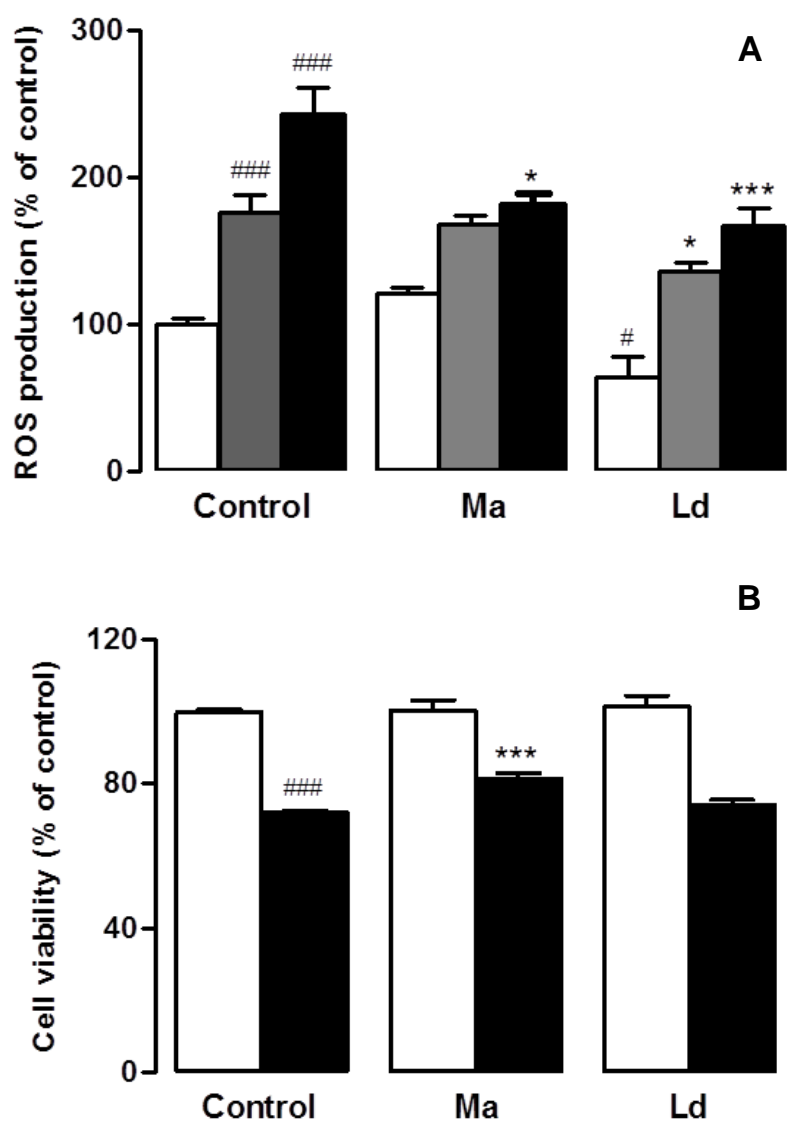

Figura 1 - Efecto protector de los extractos etanólicos de $M$. aquatica y L. dentata $(50 \mathrm{mg} / \mathrm{mL})$ contra la producción aumentada de ROS $(A)$ y la reducción de viabilidad (B) de células HepG2 en condiciones basales ( $\square, A$ y $B$ ) o en condiciones tóxicas inducidas por su incubación con dicromato potasico (DK) a $5 \mu \mathrm{M}(\mathrm{A},-1)$ y a $25 \mu \mathrm{M}(\mathrm{A}, \mathbf{m})$ durante $48 \mathrm{~h}$, o con DK a $1,5 \mu \mathrm{M}$ durante $72 \mathrm{~h}(\mathrm{~B}, \mathbf{\varpi})$. Los valores se expresan como media \pm SEM de porcentaje de la producción de ROS (A) o de viabilidad celular (B) comparación con el control, a partir de 3-4 experimentos independientes realizados por triplicado. Ma, extracto de $M$. aquatica, $L d$, extracto de $L$. dentata. ${ }^{*} p<0,05 ;{ }^{* * *} p<0,001$ en comparación con las células expuestas a $5 \mu \mathrm{M}, 25 \mu \mathrm{M}(\mathrm{A}) \circ$ $1,5 \mu \mathrm{M}$ (B) de DK, en ausencia de extracto; \# $\mathrm{p}<0,05$, \# \# \# $\mathrm{p}<0,001$ en comparación con las células no tratadas (control).

En conjunto, los resultados de esta sección proporcionan una importante información sobre la caracterización química y farmacológica de los extractos vegetales de $M$. aquatica y L. dentata y contribuyen para la valorización de estas especies. Se necesitan más estudios para aclarar la contribución exacta de los compuestos fenólicos en los efectos descritos. Se deben también llevar a cabo estudios de toxicidad para garantizar la seguridad del uso de las plantas en la prevención de trastornos relacionados con el estrés oxidativo. 


\subsubsection{Efectos protectores en células hepáticas de los compuestos fenólicos presentes en Cytisus multiflorus, Lamium album L. y Thymus $x$ citriodorus}

Dado las actividades benéficas que presentan, las plantas tienen un amplio uso en la medicina tradicional. De entre la gran diversidad de componentes de las plantas, en los últimos años se ha dado especial relevancia a los polifenoles, que son compuestos capaces de combatir el estrés oxidativo a través de distintos mecanismos. En esta parte del trabajo se evaluaron, mediante ensayos in vitro, los efectos antioxidantes y citoprotectores de extractos etanólicos purificados de las especies vegetales Cytisus multiflorus, Lamium album L. y Thymus $x$ citriodorus y de sus componentes fenólicos.

Los extractos han demostrado buenos efectos antioxidantes en las dos pruebas químicas del DPPH y del poder reductor con una orden de potencia de L. album> T. $x$ citriodorus> C. multiflorus. Además, los ensayos celulares de MTT en células humanas HepG2 indicaron que todos pueden usarse en la concentración de $50 \mu \mathrm{g} / \mathrm{mL}$ y incluso los extractos de C. multiflorus y L. album mantienen el $100 \%$ de viabilidad en concentraciones de $200 \mu \mathrm{g} / \mathrm{mL}$. Los extractos demostraron buena capacidad antioxidante, evaluada en la misma línea celular. En concreto, los extractos presentaban capacidad para neutralizar el aumento de la producción de ROS, inducido por su co-incubación con DK (Fig. 1). El extracto de $C$. multiflorus mostró una protección de 20 y 23\%(Fig. 1A), el de L. album de 23 y 26\% (Fig. 1B) mientras que el de T. $x$ citriodorus una protección de 25 y 35\%, cuando se co-incubaron con $5 \mu \mathrm{M}$ y 25 $\mu \mathrm{M}$ de DK (Fig. 1C), respectivamente. Este efecto protector también se puso de manifiesto en condiciones basales para los tres extractos. La elevada capacidad de los dos extractos para contrarrestar la formación de ROS en condiciones de estrés oxidativo en las células HepG2 está de acuerdo con la actividad de neutralización de ROS que se determinó igualmente para las mezclas de polifenoles preparadas para simular la composición fenólica predeterminada de los extractos (Fig. 1D). Además, cuando evaluamos en el mismo modelo, los compuestos puros apigenina, crisina, eriodictiol, quercetina, luteolina, naringenina, acido rosmarínico y verbascósido, todos demostraron poseer un elevado efecto antioxidante (mediado por la neutralización de ROS). 

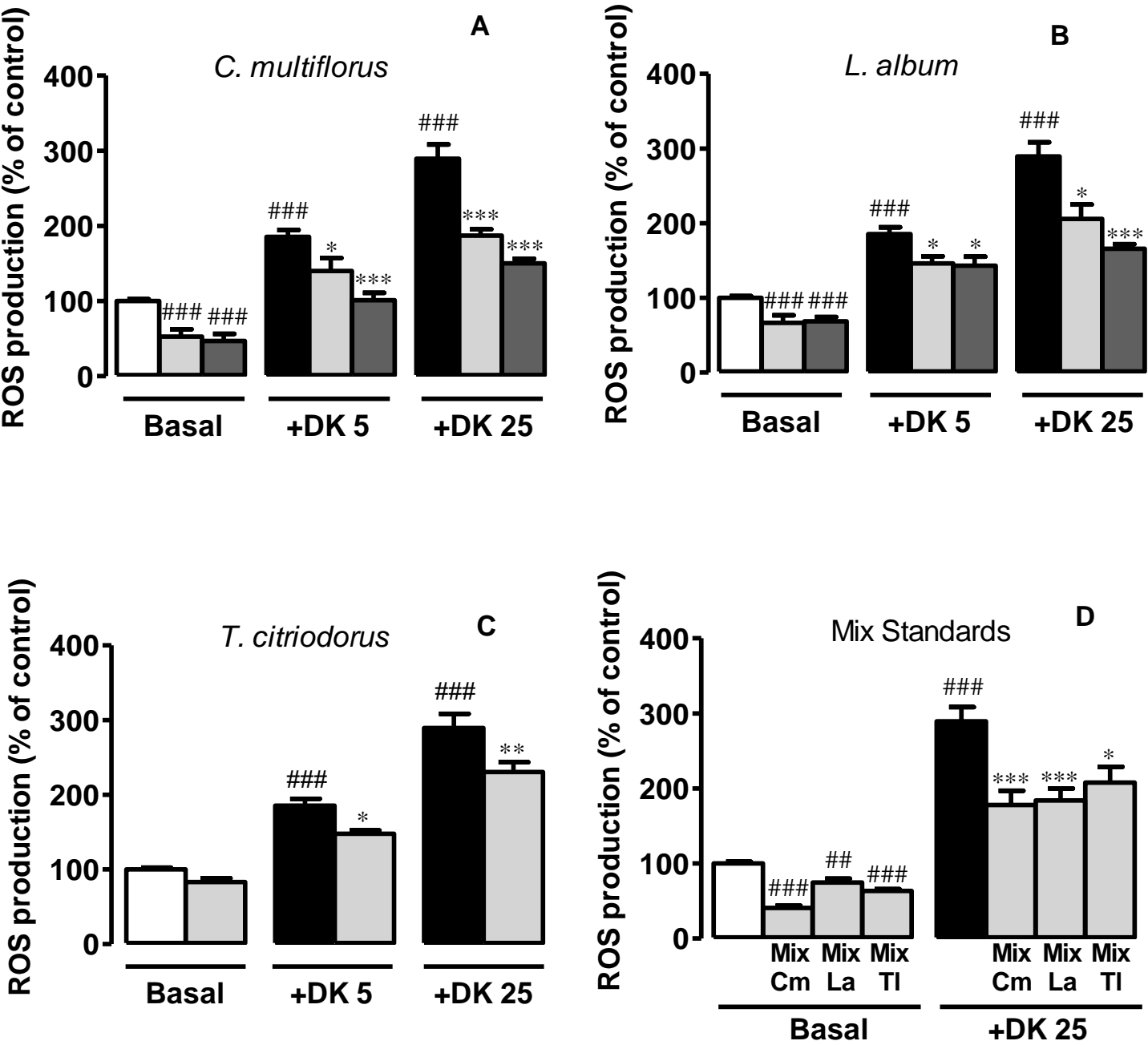

Figura 1 - Efectos protectores de extractos de Cytisus multiflorus (A), Lamium album (B) y Thymus $x$ citriodorus $(\mathrm{C})$ y de las mezclas de compuestos fenólicos que simulan su contenido en cada extracto (D) en la producción intracelular de ROS en células hepáticas HepG2, inducida por dicromato potásico (DK). Las células se incubaron en ausencia $(\square, \mathbf{\square})$ o presencia de dos dosis no tóxicas de extracto: a $50 \mu \mathrm{g} / \mathrm{mL}$ ( $\square$ ) (para los tres extractos) o a $200 \mu \mathrm{g} / \mathrm{mL}(\square)$ para los extractos de $C$. multiflorus y $L$. album y con las mezclas de compuestos fenólicos que simulan su contenido en cada extracto $(\mathrm{D},-\cdots)$. Con excepción de la condición basal las células fueron incubadas con $5 \circ 25 \mu \mathrm{M}$ de DK, por un periodo de $48 \mathrm{~h}$. Las columnas blancas ( $\square$ ) representan la condición control y las negras ( $\mathbf{a}$ ) representan la incubación de las células HepG2 solamente con DK.

El efecto citoprotector se evaluó en las células HepG2 mediante el método de medición de viabilidad celular, utilizando el test de MTT. En cuanto al potencial citoprotector de los extractos obtenidos de las especies vegetales $L$. album y $C$. multiflorus revelaron poseer un elevado efecto con 34 o 24\% de protección, respectivamente (6 h de incubación) y 11 o 12\%, respectivamente (72 h de 
incubación), como puede observarse en la Fig. 2. El efecto citoprotector del extracto de $L$. album parece estar relacionado con la presencia de verbascósido puesto que, de todos los polifenoles probados, fue el compuesto que presentó una acción citoprotectora más marcada.
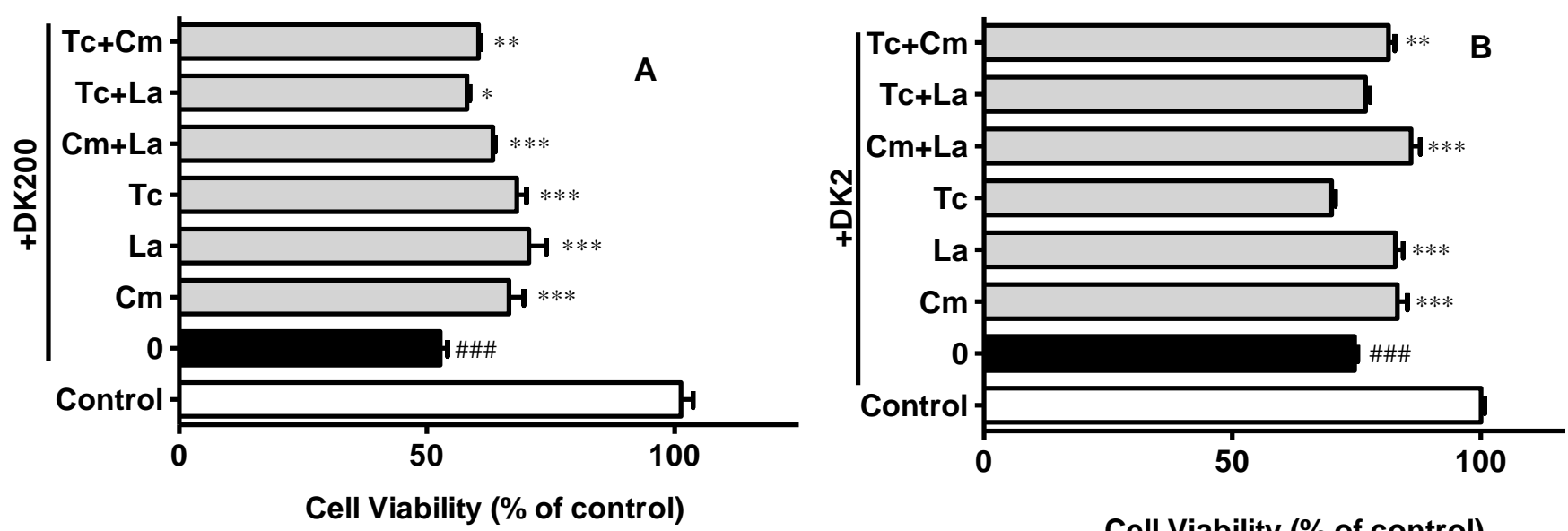

Cell Viability (\% of control)
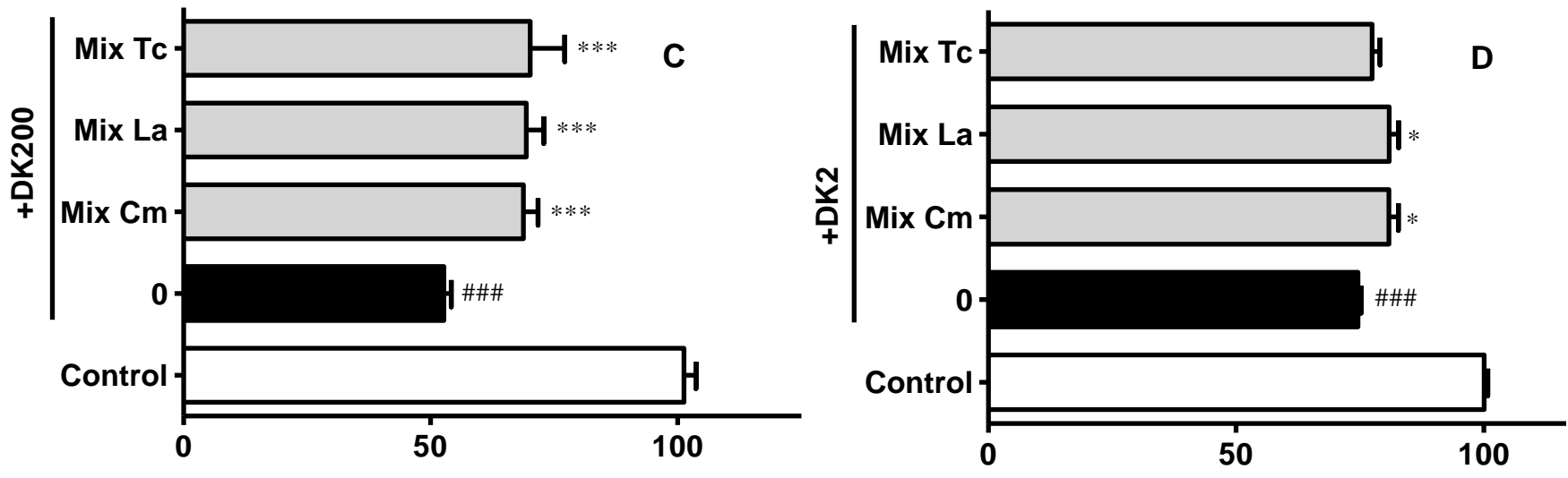

Cell Viability (\% of control)

Cell Viability (\% of control)

Figura 2 - Efectos protectores de los extractos etanólicos de Cytisus multiflorus, Lamium album y Thymus $x$ citriodorus (A, B) y de las mezclas de compuestos fenólicos que simulan su contenido en cada extracto (C, D) en un modelo de reducción de viabilidad de células HepG2 inducido por dicromato potásico (DK). Las células se incubaron en ausencia $(\square, \mathbf{\square})$ o presencia de cada uno de los extractos $(50 \mu \mathrm{g} / \mathrm{mL})$, sus mezclas $(25 \mu \mathrm{g} / \mathrm{mL}$ each) ( $\square, A, B)$ o mezclas de compuestos fenólicos que simulan su contenido en cada extracto ( $₫, \mathrm{C}, \mathrm{D})$. Excepto para en la condición control (口) las células se expusieron a $200 \mu \mathrm{M}$ de DK por un periodo de $6 \mathrm{~h}(\mathrm{~A}, \mathrm{C})$ o a $2 \mu \mathrm{M}$ por un periodo de $72 \mathrm{~h}(\mathrm{~B}, \mathrm{D})$. Las columnas negras ( $\varpi$ ) representan la incubación de las células HepG2 solo con DK. 
En general, los resultados de esta parte del trabajo sugieren que los extractos de $C$. multiflorus y $L$. album poseen buena actividad antioxidante y citoprotectora y además que los polifenoles presentes en esos extractos tienen un papel importante en las propiedades beneficiosas de estas plantas. 


\subsubsection{Efecto anti-inflamatorio de Cytisus multiflorus}

Cytisus multiflorus es un arbusto característico de la Península Ibérica, que se distribuye en la región sur-oeste del Mediterráneo. La planta se utiliza en la medicina popular porque se le atribuyen varios efectos beneficios para la salud, incluida sus propiedades anti-inflamatorias. Sin embargo, el uso de la especie vegetal $C$. multiflorus como anti-inflamatorio está basado solamente en la información etnofarmacológica, y hasta el momento no hay datos científicos que prueben la existencia de este efecto ni sus mecanismos de acción. El efecto antioxidante del extracto de $C$. multiflorus y de sus compuestos fenólicos ha sido demostrado en ensayos químicos y en células HepG2 y además se conoce que esta actividad antioxidante está asociada a las propiedades anti-inflamatorias. Por ello, el presente trabajo tuvo por objetivo aclarar los mecanismos anti-inflamatorios del extracto etanólico purificado de $C$. multiflorus. Las pruebas incluyeron el monitoreo de la actividad de especies reactivas formadas durante la respuesta inflamatoria $\left(\mathrm{HOCl}, \mathrm{NO}^{*}\right)$, así como la capacidad inhibitoria de las enzimas pro-inflamatorias clave en este proceso, como la lipoxigenasa (5-LOX), la óxido nítrico sintasa inducible (iNOS) y la ciclooxigenasa-2 (COX-2) en un modelo in vitro de inflamación.

El extracto de $C$. multiflorus mostró una protección significativa contra la producción de NO• $\left(E_{50}\right.$ de 148,0 $\left.\pm 5,2 \mu \mathrm{g} / \mathrm{ml}\right)$ más elevada que la obtenida para el ácido ascórbico, usado como control. El NO` fue igualmente medido en el modelo celular Raw 264.7 cuya producción fue inducida por lipopolisacárido (LPS). El efecto de dos concentraciones de extracto de $C$. multiflorus (160 y $325 \mu \mathrm{g} / \mathrm{mL}$ ) en la protección frente a la producción exagerada de nitritos se representa en la Fig. 1. Como puede observarse, el tratamiento de las células Raw 264.7 con LPS incrementa la producción de nitritos, lo que es inhibido en un 21 y $33 \%$ por el pre-tratamiento de los macrófagos Raw 264.7 con $160 \mu \mathrm{g} / \mathrm{mL}$ y $325 \mu \mathrm{g} / \mathrm{mL}$ de extracto, respectivamente. 


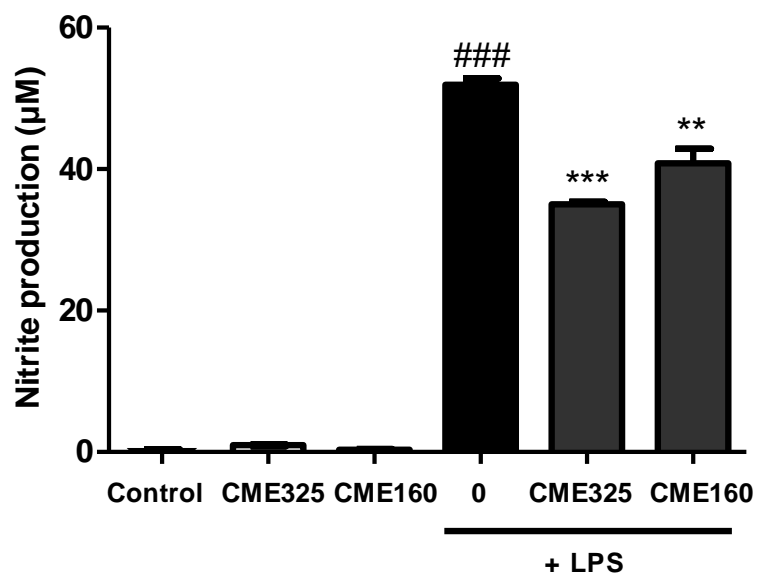

Figura 1 - Efecto del extracto de $C$. multiflorus en la producción de NO en macrófagos RAW 264.7 estimulados con $1 \mu \mathrm{g} / \mathrm{mL}$ de LPS.

Las proteínas iNOS y COX-2 juegan un papel fundamental en la inflamación ya que son, por ejemplo, responsables por la producción de mediadores pro-inflamatorios. Por ello, pareció importante medir el efecto del extracto de $C$. multiflorus en la expresión de estas enzimas, lo que se llevo a cabo por Western blot. Los resultados, que se muestran en la Fig. 2A, indicaron que la presencia del extracto fue capaz de inhibir la expresión de la enzima iNOS, de forma significativa para la concentración máxima (325 $\mu \mathrm{g} / \mathrm{mL})$, aunque no se observaron cambios en la expresión de la COX-2 (Fig. 2B).

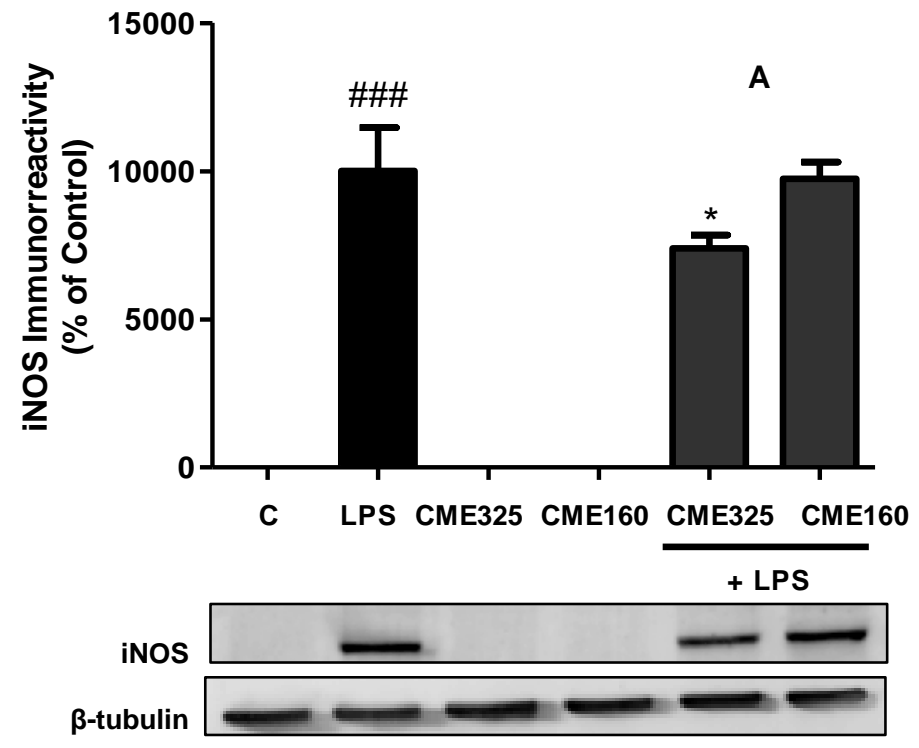




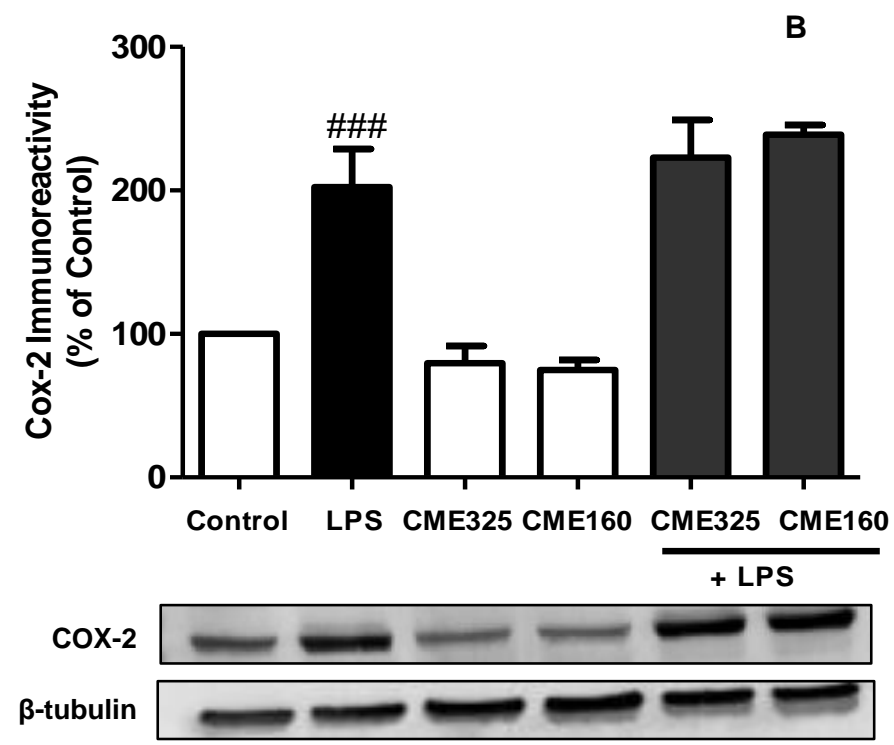

Figura 2 - Efecto del extracto de C. multiflorus a 325 y $160 \mu \mathrm{g} / \mathrm{mL}$ en el nivel de iNOS (A) COX-2 (B) en macrófagos estimulados con $1 \mu \mathrm{g} / \mathrm{mL}$ de LPS.

A pesar de no inducir cambio en los niveles intracelulares de la COX-2 se observó que el extracto a concentraciones no tóxicas fue capaz de disminuir significativamente la expresión de iNOS y también de inhibir la actividad de la enzima 5-LOX con un valor de $\mathrm{EC}_{25}$ de $37.90 \mu \mathrm{g} / \mathrm{mL}$.

Puesto que la acumulación de especies reactivas de oxígeno y nitrógeno generadas por las células inflamatorias sometidas a estrés oxidativo y la activación de las enzimas 5-LOX y iNOS son factores implicados en la inflamación crónica, los resultados apoyan el uso tradicional de $C$. multiflorus en el tratamiento de problemas inflamatorios. 


\subsubsection{Influencia de los extractos etanólicos purificados de Mentha aquatica L. y Leonurus cardiaca L. en la bioenergética mitocondrial}

La mitocondria presenta un papel esencial en la homeostasis celular, una vez que participa en la síntesis de ATP a través de la fosforilación oxidativa, y en la biosíntesis de ácidos grasos y aminoácidos. Además de las funciones metabólicas, la mitocondria está implicada en los flujos de calcio, producción de ROS y RNS y señalización celular. La mitocondria es también un reconocido modelo para evaluación de la toxicidad celular de xenobióticos es y igualmente utilizada como biosensor para predecir la seguridad de fármacos. Las perturbaciones en la bioenergética mitocondrial están relacionadas con distintos mecanismos asociados con lesión celular y varias disfunciones. Por su enorme importancia en la célula, la mitocondria es también una potencial diana para fármacos, con lo que hay incluso fármacos usados como antidiabéticos, antivirales, antitumorales en los que el mecanismo de acción está basado en alteraciones de funciones mitocondriales.

De esta manera, esta última parte del trabajo tenía como objetivo evaluar los posibles efectos de los extractos etanólicos de $M$. aquatica y $L$. cardiaca en la bioenergética mitocondrial. Para ello, se realizaron ensayos para medición de parámetros mitocondriales como el estado 3 , el estado 4, la relación del control respiratorio respiración no acoplado $(\mathrm{RCR})$ y la relación de $\mathrm{P} / \mathrm{O}$, además de la evaluación del potencial de membrana, en presencia de dos concentraciones distintas de los extractos.

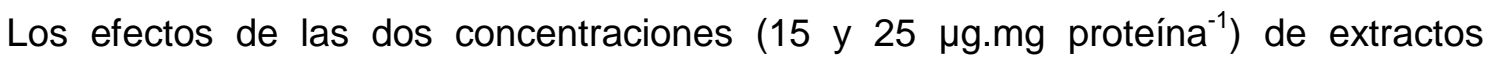
etanólicos de $M$. aquatica y L. cardiaca en los estados metabólicos de mitocondrias aisladas de hígado se presentan en la Tabla 1. 
Tabla 1 - Efectos de los extractos de M. aquatica (PEEMa) y L. cardiaca (PEELc) en las tasas respiratorias de mitocondrias de hígado.

\begin{tabular}{|c|c|c|c|c|c|}
\hline Extracto & Condición & $\begin{array}{c}\text { V2 } \\
\mathrm{nmol} \mathrm{O}_{2} \cdot \mathrm{mg}^{-} \\
{ }^{1} \cdot \mathrm{min}^{-1}\end{array}$ & $\begin{array}{c}\text { V3 } \\
\mathrm{nmol} \mathrm{O}_{2} \cdot \mathrm{mg}^{-} \\
{ }^{1} \cdot \mathrm{min}^{-1}\end{array}$ & $\begin{array}{c}\text { V4 } \\
\mathrm{nmol} \mathrm{O}_{2} \cdot \mathrm{mg}^{-} \\
{ }^{1} \cdot \mathrm{min}^{-1}\end{array}$ & 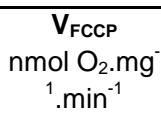 \\
\hline \multirow{8}{*}{ PEEMa } & Glutamato + Malato & & & & \\
\hline & Control & $4,2 \pm 0,5$ & $40,5 \pm 1,9$ & $10,9 \pm 0,9$ & $42,6 \pm 1,2$ \\
\hline & $\begin{array}{l}\text { M. aquatica } \\
15 \text { mg.mg protein }{ }^{-1}\end{array}$ & $4,9 \pm 0,9$ & $35,0 \pm 1,5$ & $9,6 \pm 0,7$ & $32,0 \pm 1,6$ ** \\
\hline & $\begin{array}{l}\text { M. aquatica } \\
25 \mu \mathrm{g} \cdot \mathrm{mg} \mathrm{protein}^{-1}\end{array}$ & $4,7 \pm 0,4$ & $30,7 \pm 1,8^{*}$ & $9,8 \pm 0,5$ & $24,9 \pm 1,9^{\star *}$ \\
\hline & Succinato & & & & \\
\hline & Control & $6,3 \pm 0,3$ & $26,1 \pm 1,0$ & $6,3 \pm 0,3$ & $32,6 \pm 1,9$ \\
\hline & $\begin{array}{l}\text { M. aquatica } \\
15 \mu \mathrm{g} . \mathrm{mg} \mathrm{protein}^{-1}\end{array}$ & $7,1 \pm 0,4$ & $23,6 \pm 1,1$ & $6,4 \pm 0,4$ & $25,4 \pm 3,4$ \\
\hline & $\begin{array}{l}\text { M. aquatica } \\
25 \mu \mathrm{g} . \mathrm{mg} \mathrm{protein}^{-1}\end{array}$ & $7,0 \pm 0,4$ & $21,8 \pm 1,25^{\star}$ & $6,5 \pm 0,4$ & $22,5 \pm 3,1^{*}$ \\
\hline \multirow{8}{*}{ PEELc } & Glutamato+ Malato & & & & \\
\hline & Control & $4,1 \pm 2,0$ & $17,8 \pm 2,1$ & $4,4 \pm 0,4$ & nd \\
\hline & $\begin{array}{l}\text { L. cardiaca } \\
15 \mu \mathrm{g} \cdot \mathrm{mg} \mathrm{protein}^{-1}\end{array}$ & $4,8 \pm 0,6$ & $18,9 \pm 2,8$ & $4,5 \pm 0,5$ & nd \\
\hline & $\begin{array}{l}\text { L. cardiaca } \\
25 \mu \mathrm{g} . \mathrm{mg} \mathrm{protein}^{-1}\end{array}$ & $4,6 \pm 0,7$ & $18,6 \pm 3,0$ & $4,5 \pm 0,4$ & nd \\
\hline & Succinato & & & & \\
\hline & Control & $6,3 \pm 0,3$ & $25,0 \pm 1,1$ & $5,5 \pm 0,2$ & $36,6 \pm 0,9$ \\
\hline & $\begin{array}{l}\text { L. cardiaca } \\
15 \mu \mathrm{g} . \mathrm{mg} \mathrm{protein}^{-1}\end{array}$ & $7,2 \pm 0,2$ & $22,3 \pm 1,1$ & $5,9 \pm 0,2$ & $35,7 \pm 0,3$ \\
\hline & $\begin{array}{l}\text { L. cardiaca } \\
25 \mu \mathrm{g} \cdot \mathrm{mg} \mathrm{protein}^{-1}\end{array}$ & $7,1 \pm 0,4$ & $20,3 \pm 1,1^{*}$ & $6,4 \pm 0,2^{*}$ & $31,7 \pm 1,6^{\star \star}$ \\
\hline
\end{tabular}

Nd- No se determinó

Se observó una disminución dosis-dependiente, en el estado respiratorio 3, en presencia de los dos substratos usados, para el extracto de M. aquatica. El estado respiratorio 4 no se modificó significativamente. Por otro lado, el tratamiento de las mitocondrias con las mismas concentraciones de extracto de $L$. cardiaca indujo un descenso del estado 3 y un aumento del estado 4. De esta forma, se detectó un descenso en el RCR que estaba afectado directamente por esta disminución en el estado 3 (para el glutamato/malato en presencia del extracto de $M$. aquatica y para el succinato en presencia de ambos extractos), seguido por un aumento de estado 4 (en succinato estimulada por las mitocondrias en la presencia de L. cardiaca). Sin embargo, los extractos no inducían cambios en el la razón ADP/O, lo que indica que la eficiencia del sistema fosforilativo no estaba afectada. 
Tabla 2 - Efectos de los extractos de M. aquatica (PEEMa) y L. cardiaca (PEELc) en el potencial de membrana mitocondrial.

\begin{tabular}{|c|c|c|c|c|c|c|}
\hline Extracto & Condición & $\begin{array}{l}\text { Energizacion } \\
\qquad(\mathrm{mV})\end{array}$ & $\begin{array}{c}\Delta \text { ADP1 } \\
(\mathrm{mV})\end{array}$ & $\begin{array}{l}\text { Rep } \\
(\mathrm{mV})\end{array}$ & $\begin{array}{c}\text { Vrep } \\
\text { (\% of mean } \\
\text { control) }\end{array}$ & $\begin{array}{c}\text { Lag phase } \\
\text { (s) }\end{array}$ \\
\hline & Glutamato + Malato & & & & & \\
\hline & Control & $209,3 \pm 1,5$ & $26,9 \pm 2,0$ & $205,7 \pm 1,2$ & $100,0 \pm 6,0$ & $46,7 \pm 1,2$ \\
\hline & $\begin{array}{l}\text { M. aquatica } \\
15 \mu \text { g.mg protein }{ }^{-1}\end{array}$ & $202,9 \pm 2,3$ & $27,1 \pm 2,1$ & $200,3 \pm 2,4^{*}$ & $84,8 \pm 5,0$ & $55,5 \pm 1,0^{*}$ \\
\hline & $\begin{array}{l}\text { M. aquatica } \\
25 \mu \text { g.mg protein }{ }^{-1}\end{array}$ & $200,8 \pm 2,4^{*}$ & $25,8 \pm 1,4$ & $199,9 \pm 1,3^{*}$ & $75,5 \pm 5,0^{*}$ & $65,5 \pm 3,2^{* *}$ \\
\hline \multirow[t]{7}{*}{ PEEMa } & Succinato & & & & & \\
\hline & Control & $218,8 \pm 1,0$ & $29,0 \pm 1,1$ & $218,4 \pm 1,1$ & $100,0 \pm 5,7$ & $66,6 \pm 5,1$ \\
\hline & $\begin{array}{l}\text { M. aquatica } \\
15 \mu \mathrm{g} . \mathrm{mg} \mathrm{protein}^{-1}\end{array}$ & $218,3 \pm 0,9$ & $28,5 \pm 2,4$ & $216,7 \pm 1,6$ & $91,4 \pm 4,3$ & $64,0 \pm 6,6$ \\
\hline & $\begin{array}{l}\text { M. aquatica } \\
25 \mu \text { g.mg protein }{ }^{-1}\end{array}$ & $215,0 \pm 1,2^{*}$ & $27,9 \pm 1,3$ & $214,1 \pm 1,4^{*}$ & $75,7 \pm 3,4^{\star \star}$ & $76,6 \pm 5,0^{*}$ \\
\hline & Glutamato + Malato & & & & & \\
\hline & Control & $212,4 \pm 1,6$ & $28,4 \pm 0,8$ & $210,7 \pm 1,6$ & $100,0 \pm 4,3$ & $55 \pm 2,7$ \\
\hline & $\begin{array}{l}\text { L. cardiaca } \\
15 \mu \mathrm{g} . \mathrm{mg} \mathrm{protein}^{-1}\end{array}$ & $208,1 \pm 1,7$ & $27,9 \pm 1,1$ & $205,6 \pm 1,7$ & $104,0 \pm 3,7$ & $53,8 \pm 4,8$ \\
\hline \multirow[t]{5}{*}{ PEELc } & $\begin{array}{l}\text { L. cardiaca } \\
25 \mu \mathrm{g} \cdot \mathrm{mg} \text { protein }{ }^{-1}\end{array}$ & $206,5 \pm 2,1$ & $24,3 \pm 0,7^{\star}$ & $204,4 \pm 2,1^{*}$ & $82,8 \pm 4,2^{*}$ & $53,2 \pm 2,0$ \\
\hline & Succinato & & & & & \\
\hline & Control & $217,1 \pm 1,1$ & $32,6 \pm 1,0$ & $217,0 \pm 1,1$ & $100,0 \pm 4,1$ & $58,1 \pm 1,6$ \\
\hline & $\begin{array}{l}\text { L. cardiaca } \\
15 \mu \mathrm{g} \cdot \mathrm{mg}_{\text {protein }}{ }^{-1}\end{array}$ & $213,2 \pm 1,0^{*}$ & $35,4 \pm 1,0$ & $212,2 \pm 1,0^{*}$ & $86,1 \pm 3,5^{\star}$ & $72,6 \pm 2,0^{* *}$ \\
\hline & $\begin{array}{l}\text { L. cardiaca } \\
25 \mu \text { g.mg protein }{ }^{-1}\end{array}$ & $212,8 \pm 0,8^{*}$ & $28,3 \pm 1,2^{*}$ & $211,3 \pm 0,9^{\star *}$ & $68,6 \pm 3,4^{\star \star \star}$ & $81,0 \pm 3,7^{\star \star \star}$ \\
\hline
\end{tabular}

El tratamiento de las mitocondrias con las dos concentraciones de los extractos de $M$. aquatica y $L$. cardiaca condujo a una disminución progresiva del $\Delta \Psi$ máximo, independientemente del sustrato respiratorio utilizado (Tabla 2). Se observaron diferencias estadísticamente significativas para la concentración de extracto de $M$. aquatica más alta, en ambos substratos. Para el extracto de $L$. cardiaca este efecto fue evidente para el substrato succinato, además de inducir un descenso en la amplitud de despolarización, posterior a la adición de ADP. Los resultados también indicaron que los dos extractos $\Delta \Psi$ interfieren con la repolarización. Después de la adición de ADP, en presencia de los extractos, las mitocondrias nunca fueron capaces de recuperar $\Delta \Psi$ para los valores de control. En la mayoría de las condiciones ensayadas, se observó un aumento de la lag phase, lo que es coincidente con el descenso del estado 3. 
En resumen los resultados indican que los extractos disminuyen la relación del control respiratorio: el extracto de $M$. aquatica induce una disminución en el estado respiratorio 3 mientras que el extracto de $L$. cardiaca afecta a los estados respiratorios 3 y 4 . Los extractos de $M$. aquatica y $L$. cardiaca afectan a la funcionalidad de las mitocondrias de hígado, sin afectar su eficiencia fosforilativa. 


\subsection{CONCLUSIONES}

\section{CONCLUSION PRIMERA}

HPLC-DAD combinado con los métodos ESI-MS ${ }^{n}$ en el modo negativo y el NMR son técnicas analíticas útiles para la caracterización fenólica de los extractos etanólicos obtenidos a partir de las especies vegetales Cytisus multiflorus, Lamium album L., Lavandula dentata L., Leonurus cardiaca L., Mentha aquatica L. y Thymus $x$ citriodorus.

\section{CONCLUSION SEGUNDA}

Los extractos etanólicos de las especies de plantas que han sido objeto de este trabajo presentan distintos perfiles fenólicos. Mientras que los de C. multiflorus y T. $x$ citriodorus son abundantes en flavonas, los de $M$. aquatica contienen altas cantidades de flavanonas (como derivados glucósidos de eriodictiol, naringenina y hesperitina). Por su parte, los extractos etanólicos de L. dentata contienen casi exclusivamente ácido rosmarínico, mientras que los de $L$. album y $L$. cardiaca son ricos en feniletanoides glucósidos.

\section{CONCLUSION TERCERA}

Los ensayos químicos de determinación del potencial antioxidante DPPH y poder reductor indican que los seis extractos de plantas poseen una elevada actividad antioxidante. Los cuatro extractos antioxidantes más relevantes mostraron la orden de potencias de $M$. aquatica $>L$. album $>L$. dentata $>T$. $x$ citriodorus. Los valores de $E_{50}$ de los seis extractos de plantas tuvieron una variación desde 8,1 hasta 18,3 $\mu \mathrm{g} / \mathrm{mL}$ y 51,9 a $95,7 \mu \mathrm{g} / \mathrm{mL}$ para el efecto bloqueador de radicales de $\mathrm{DPPH}^{\bullet}$ y para el poder reductor, respectivamente.

\section{CONCLUSION CUARTA}

Los extractos obtenidos de las plantas medicinales C. multiflorus, L. album, T. $x$ citriodorus y $L$. dentata son capaces de contrarrestar eficazmente el aumento de la formación de ROS en modelos de estrés oxidativo en las células hepáticas HepG2. 


\section{CONCLUSION QUINTA}

Los extractos etanólicos de L. album, C. multiflorus, T. $x$ citriodorus y M. aquatica poseen efectos citoprotectores en células hepáticas HepG2 en condiciones de estrés oxidativo.

\section{CONCLUSION SEXTA}

Las actividades antioxidante (ROS-scavenging) y citoprotectora de los extractos de $C$. multiflorus, L. album, T. $x$ citriodorus están estrechamente relacionadas con su contenido en compuestos fenólicos.

\section{CONCLUSION SÉPTIMA}

El extracto de C. multiflorus posee actividades anti-inflamatorias mediadas por la captura del radical $\mathrm{NO}^{\bullet}$ y por el descenso de la expresión de la enzima iNOS, así como por la inhibición de la actividad de la enzima 5-LOX.

\section{CONCLUSION OCTAVA}

Los extractos de $M$. aquatica y $L$. cardiaca afectan a la funcionalidad de mitocondrias de las células hepáticas. El extracto de $M$. aquatica induce una disminución en el estado respiratorio 3 , mientras que el extracto de $L$. cardiaca afecta a los estados respiratorios 3 y 4 . Ambos extractos disminuyen la relación del control respiratorio sin afectar la eficiencia fosforilativa de las mitocondrias.

\section{CONCLUSION GENERAL}

La especies C. multiflorus, L. album, L. dentata, L. cardiaca, M. aquatica y T. $x$ citriodorus son buenas fuentes de compuestos fenólicos. Teniendo en cuenta el papel fisiopatológico del estrés oxidativo en distintas enfermedades, junto con la alta capacidad antioxidante demostrada por la mayoría de los extractos estudiados, se pueden proponer como potenciales agentes preventivos. Además, parece ser que la especie C. multiflorus puede ser útil en el tratamiento de trastornos asociados a procesos inflamatorios. 